QL

AGE PUBLIE SOUS LES AUSPICES NISTERE DE LINSTRUCTION PUBLIOQUE

SOUS LA DIRECTION DE L. JOUBIN PROFESSEUR AU MUSEUM D'HISTOIRE NATURELLE

\title{
DEUXIEME EXPÉDITION
}

\section{ANTARCTIQUE FRANÇAISE}

$(1908-1910)$

COMMANDÉE PAR LE

\section{$D^{\mathrm{r}}$ JEAN CHARCOT}

SCIENCES NATURELLES : DOCUMENTS SCIENTIFIQUES

\section{EXTRAIT}
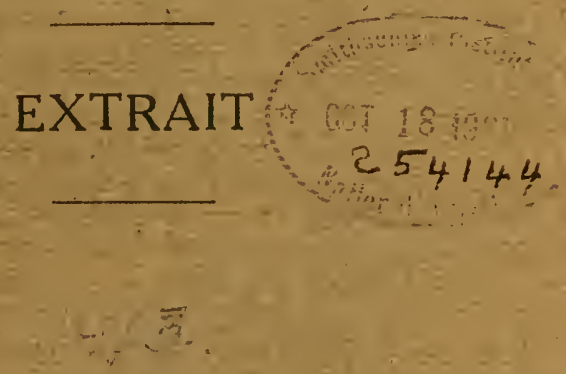

MASSON ET C ${ }^{1 \%}$, EDITEURS 120, Bd SAINT-GERMAIN, PARIS (VI०)

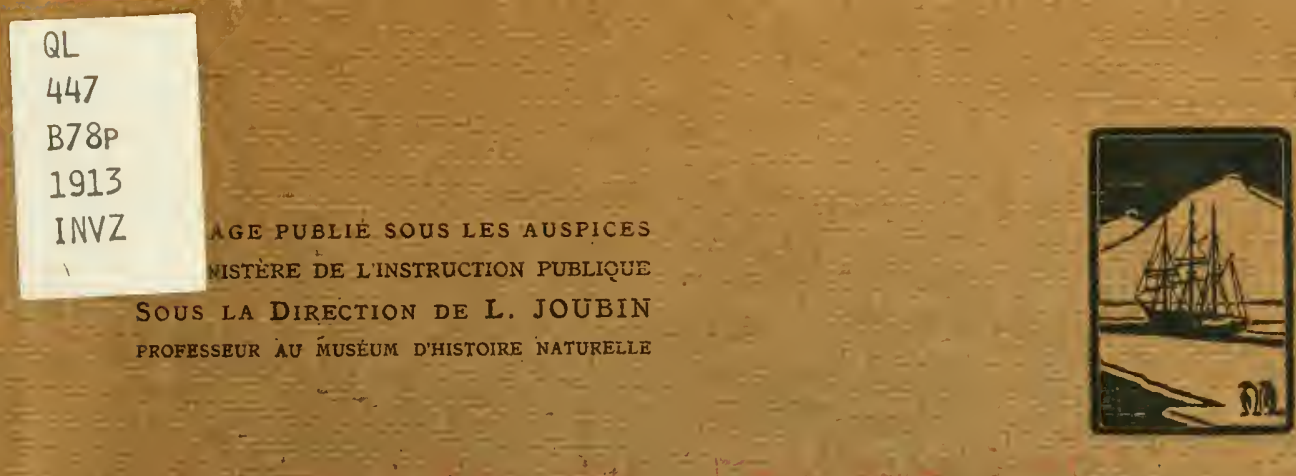

- 
QL

447

$B 78 \mathrm{P}$

1913

INVZ

AGE PUUBLIE SOUS LES AUSPICES NISTERE DE LINSTRUCTION PUBLIOYUE

SOUS LA DIRECTION DE L. JOUBIN PROFESSEUR AU MUSEUUM D'HISTOIRE NATURELLEE

DEUXIEME EXPEDITION ANTARCTIQUE FRANÇAISE $(1908-1910)$ COMMANDÉE PAR LE. $D^{\mathrm{r}}$ JEAN CHARCOT

SCIENCES NATURELLES : DOCUMENTS SCIENTIFIQUES

EXTRAIT

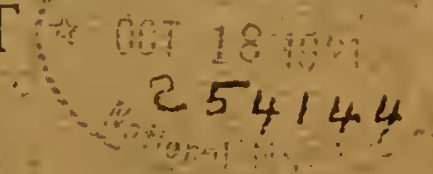

MASSON ET C'R, EDDITEURS 120, Bd SAINT-GERMAIN, PARIS (VI*) 


\section{Commission Chargé par L'ACAdémie des SCIEnces}

d'élaborer le programme scientifique de l'Expédition

MNi. les Membres de l'Institut :

\begin{tabular}{l|l|l|l} 
Bovelet de la Grye. & Glard. & DE Lapparext. & Müntz. \\
Bornet. & GuYou. & MangiN. & Ed. Perrier. \\
Bouvier. & Lacroix. & Mascart. & Roux. \\
Gaudry. & &
\end{tabular}

Commission nomiée par le Ministère de l'Instruction Publique pour examiner les résultats scientifiques de l'Expédition

iMM. Ed. Perrier.......... Membre de l'Institut, Directeur du MIuséum d'Histoire naturelle, Président.

Vice-Amiral Foursirr, Aiembre du Bureau des Longitudes, Vice-Président.

A.got............ Directeur du Bureau central météorologique.

Bayet............ Correspondant de l'Institut, Directeur de l'Enseignement supérieur.

Bigolrdan .......... Membre de l'Institut, Astronome à l'Observatoire de Paris.

Colonel Bourgeors.... Directeur du Service géographique de l'Armée.

Bouvier........... Membre de l'Institut, Professeur au Muséum d'Histoire naturelle.

Gravier........... Assistant au Muséum d'Histoire naturelle.

Commandant Guyou.. Membre de l'Institut, Membre du Bureau des Longitudes.

HANusse........... Directeur du Service hydrographique au Ministère de la Marine.

Jovbix............. Professeur au Mfuséum d'Histoire naturelle et à l'Institut Océanographique.

Lacrolx ............ Membre de l'Institut, Prolesseur au-Muséum d'Histoire naturelle.

Laliemand.......... Membre de l'Institut, Membre du Bureau des Longitudes, Inspecteur général des mines.

Lipipanix . ........ Membre de l'Institut, Professeur à la Faculté des Sciences de l'Université de Paris.

Müмtz ............ Membre de l'Institut, Professeur \& l'Institut agronomique.

Razot............. Membre de Ia Commission des Voyages et Missions scientifiques et littéraires.

Rotx ............. Membre de l'Institut, Directeur de l'Institut Pasteur.

VÉlais ............ Professeur à la Faculté des Sciences de l'Uniniversité de Paris. 


\section{PYCNOGONIDES DU " POUROUOI PAS"”"}

Par M. E.-L. BOUVIER

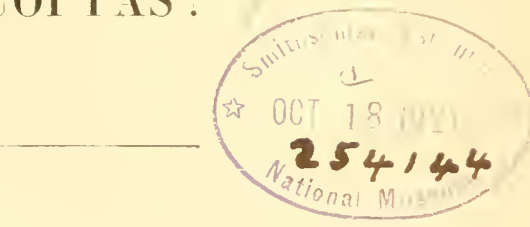

La faune des Pycnogonides antaretiques est heaucoup plus riche et plus intéressante qu'on n’avait pu le croire tout d'abord. Longtemps restée inconnue comme les mers qu'elle habite, les premières tentatives faites pour l'étudier ne remontent guère au delà d'un demi-siècle et ne firent connaitre qu'un petit nombre de formes, pour la plupart subantaretiques. La campagne du "Challenger " accrut dans des proportions notables ce faible contingent, sans toutefois donner l'espoir de récoltes ultérieures très abondantes. Mais, depuis dix ans. Iesexpéditions scientiliques se son multipliées dans les mers australes, et chacune d'elles a singulirrement enrichi le catalogue des Pyenogonides antaretipues: la dernirpe en date, celle du "Pourquoi l'as? ", ne le è̀de en rien à la plus fructueuse des expéditions précédentes; d'un bond, elle a donné aux Pycnogonides antarctiques la prépoudérance sur ceux des mers boréales, si bien qu'on peut se demander aujourd'hui où s'arrêtera les richesses d'une faune que touts campagne nouvelle accroit dans de si grandes proportions.

Pour fixer cette date et pour donner une justification à ces espoir's, je crois utile de résumer brièvement les travaux consacrés jusfpu'ici aux Pycnogonides des mers australes. Dans cet examen historique, je suivrai l'exemple donné par H. Hodgson 1907", 1908 en divisant ces mers en une région antarctique et uno région subantaretique. Ces deux régions confluent à $60^{\circ}$ de latitude sud, la seconde ayant pour limite septen-

I) La nomenclature des diverses parties du corps et les appendices, dans le présent travail, est celle que j’ai adoplée dans mon étule sur les Pycnogonides du "Français " 1906 b. p. 5 et 6); loutefois, pour abréger dans les tableaux de mensurations, jai désigné sous le nom de céphalothorax la partie du corps quí comprend le céfhalon censemble des somites prépédiféres el le tronc (somites pédifères). Celte dénomination n’est pas anssi vicieuse çu on pourail le croire, car, chez beaucoup de Pýcnogonides, les somitesdu tronc ne sont pas articulés et forment un lout continu arec le céphalon. 
trionale, comme l'admet M. Buchan (1), l'isotherme de $45^{\circ} \mathrm{F}$. (environ 7o C.), qui passe un peu an nord d'Arekland et des iles Falliand, des Crozet et de Kerguelen, dans une dircetion à très peu près parallèle à l'équateur.

Je erois bon également de liviser, comme If. Hodgson, la région subantaretique en troisprovinces: la prorince magellenique comprise entre $20^{\circ}$ el 1300 long. O. G. ; la prominre anstralienne, entre 130 long. O. Li. et 1000 Song. E. G.; pnlin la prominerafircaine on de kerguelen, qui s’étend sur le reste de la région subantaretique de $100^{\circ}$ long. E. G. à $20^{\circ}$ long. (1. G.).

Les Pycnogonides antarctiques jusqu'à l'expédition du "Pourcuoi pas?". - Ciest par une découverte de prenier ordre que s'affirme le premirp travail relatif aux Pyenogonides antarctiques. En 183 i, dans un mémoire très explicite el aceompagné de figures birn nettes, J. Eakitic 1834, 203-206, Pl. VII décrivit sous le nom de Decolopoda austratis un l'yenogonide nouvan of de grande taille, qui différait de foutes tes espiens juspùalors comnnes dans le groupe par la présenee de cinq paires dre pattes an lieu de quatre. qui est le nombre normal. Ce mémoire resta iniperé juscu'en 190:i, éjoque oi M. Hodgson 1905 a) le signala anx zoologistes. Mans son grand travail sur les Pyenogonides du "Challengar ", M. IInEk $1881^{a}, 6$ ) ne le eite que d'après l'édition anglaise du Regue animal de Cuvier et semble ne pas avoir pu se le procurer :

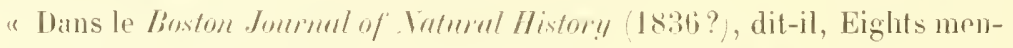
tionne le genre Decoloporda, mais je n'ai ju savoir si ce genre est valable, ni oủ il a étí trouví. " En lait, les exemplaires de James Eights avaient été captnrés aux shetlands du sud, ainsi qu il est dit dans le mémoire de l'anteur. On verra plus loin que l'espèce existe tonjours dans cette région at qu'elle se lrouve en outre aux Orades du Sud.

Il nous fint maintenant franclur une longue période, près d’un demisièche, four tronver d'autres documents relatif's à la lame des Pyenogonides intaretipues.

Eu 187:, Lm. Mn:m signale brièrement quelques Pyenogonides subanLanctiques recueillis à Kergnelen par A. E. Eaton, le Nymephon gracilipes nov. et le $N$. styligenum nov. $\left(1875^{a}, 76\right)$, auxquels vient s'ajouter une

(1) A. Bucnax, Rejort on atmospheric circulation based on the observalions made on board II. M. S. "Challenger" (Challenter, Phys, ant. Chem., vol. MI, 1889). 
troisième espèce, le $N$. lreviraudatmu nov. $\left(1875^{b}\right)$. Ces trois l'yenogonides furent figurés et plus explicitement décrits en 1879 par le mème auteur (1879, 211, P. X1), qui ́́talslit le genre Tungstylum pour son 1. styligermm. Les teux autres espices furent laissees dans le gamre Tymphon, mais on verra plus loin que le $\lambda$. bervirtmdatum appartient an genre Chrefomyutphum, phus tard établi par M. (1. 0. Sars.

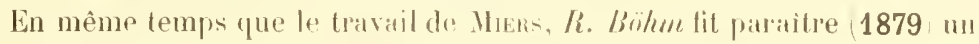
mémoire ronsacré atıx Pyrnogonides dn Muséum de Berlin el principa-

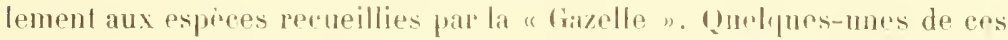

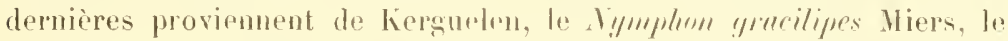

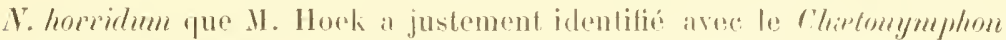
mevicamblum Hiors. I'Arhelin lime Hodge qui est ume Achélie probablement distincte de l'espicer établie par Hodge, entin un prétendu Pycrogommu littornle Strön, dont Möbiusa fait, datrs lat suite, le type d'une espèce nouvelte, le P. murguimostir. Böhm signale aussi en l'atagonie et dans le détroit de Magrellan la Prnllene fluminemsis Kröyes, qui est une l'allenopsis. Le mémoire de Böhm est concis et atreompagné de homnes figures.

Nous voici arrivés en 1881, époque oil 11 . Hoek (18819) publia son remarquable travail sur les Pycuogonides recueillis par le "Challenger" . Cette étude passe en rerne fontes les esperes du grompe jusqualors signalées: elle en fixe la synonymie et les répartit cu fanille suivant un système de classifiration proposí par l'auteur. Jusqu'alor's les espèces connues dansles mers froidesanstratesétaient an nombre de 7, toutes subantaretiques à l'exeeption de la Iteentoportur austratis; du coup, ce nombre est portí à 21 sans aecroitre d'ailleurs la faune de la région antaretipue vraie, toujours réduite à la seule Drentrpunta. Ainsi les espicens capturées par le "Challenger", daus lesmers australes sont tontes subautaretiques; la plupart proviennent de la province afrieaine, des eaux de kerguelen ou elles ont dité prises dans la région sublittorale, Vymphon hrachyrhynchus

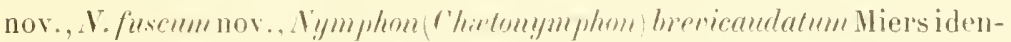

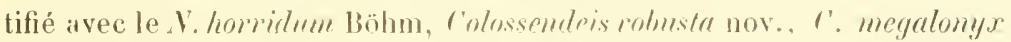
nov. trouvée aussi dans la provinee de 11 agellan ; les autres furent prises dans les régions abyssales, principalement au voisinage des Crozet, entre 
le Cap et Kerguelen Wymphom hamatum nov., Ascorhynrhus glaher nov., Colossendris giggs nov., ''. Ieptorhynehe nov., '. gigus-leptorhynch" nov.. C. araeilis nov.., et Phorichilidium (P'allenopsis) pilosnm nov., que l'expródition a également recueilli dans la province australienne]. Les autres provinces subantaretiques sont moins bien partagées dans les réeoltes du "Challenger" : celle de Magellan s'enrichit de deux espèces sublittorales, te Phorrichilidimu Pallenopsis) patagonicm nor. et la Folossendeismegalomy.r trouvée aussià Kerguelen; la proviner australienne, de deux espèces abyssales, le Vymphon meridionale nov. et le Phoxichiliflimm (Pullenopsis) pilosmm capturé anssi en province africaine. A cette liste, il convient d'ajouter le Nymphon compachm nov, recueilli par 1100 brasses près d'Aucklaud el plus tard retrouvé par la "Scotia " en région antarctique, et le I'hurirhilidium Pallenopsis) fluminense Kóryer, signalé par Böhnn en l'atagonie et retrouvé par le "Challenger " ¿ Bahia. Cette dernir̀re espice est sublittorale, par conséquent peu sensible aux différences de tempírature; toutes les autres recherchent les eaux froides ou presque froides et, des lors, sont capables de se répandre dans les nors antaretirues.

Les seize espirees précédentes no sont pas les senles eapturées par le "Lhallengre" dans la récion subantaretique; trois autres furent décrites ot lizurées beaucoup plus tard, en 1898, dans un mémoire spécial dû crncor'r à la plume experto de 11 . Hork (1898, 290-295, Pl. 11 et III) : le Pyonoyommm mayellanirmm nov., des parages de Magellan, la Pallene dimorphu nov. de kirguclen et l'Amplodurtylus neglecta nor., trouvée entre les iles cirozet el l'ile du Prince-Edwards. Les denx premières habitent la région sublittorale, la troisicme est franchement abyssale.

Les recherches du lieutenant italien li. Chierchia, effectuées is bord de la corvelte "Vettor P'isani ", enrichissent la faune subantarctique d'une espèce nouvelle, te Trunstylum Chirerhie, signalée par M. W. ScmukeWitcul dans une notice préliminaire 1887, 271, puis longuement décrite et ligurée par le mème auteur dans un mémoire spécial 1889, 333, fig. 8-11). Cette espèce fut trouvée aux îles Chonos. La même expédition recueillit aux Abrolhos et dans le détroit de Hagellan la P'ullemopsis fluminmsis Kröyer, déjà signalée par bölım dans cette dernière région. 
En mème temps que II. Schimkewitsch publiait son mémoire, M. (i. P'feffen consacrait une étude sureinete et sans figures aux Pyenogonides antarctiques de la Géorgie de sud $(\mathbf{1 8 8 9}, 41-49)$. Les espères décrites par l'anteur sont 1. Nymphon anturetienm nov., lo Vymphon (Chetonymphom) hrevicandatum. Viers déjà signali aux Kergnelen, l'Ammothen grande nov. et l'A. Clausii nor., l'A. Hoplizi, dont on doit faire, ee me semble, une Ammothella, ear ses palpes comprenuent neuf articles; entin la Clotenin Dohmii, que je considère, avec II. IIodgson (1907, 1908a), comme un Tunystylum. Il est bon de rappeler à ce propos que M. Schimkewitsch, en 1887 et 1889 , fit connaitre sous le nom de Turystylum Dolurnii nov. une espece trouvée par le lieutenant Chierchia anx iles Abrollos ; la Clotenia de $1 \mathrm{l}$. Pfeffer, étant un Tramystylmm, ne saurait conserver le qualilicatil spécilique Dohrmii que lui domna l'auteur; je propose d'attribuer à cette espèce le nom de Tumysty/mm Pfefferi en souvenir du savant qui l'a décrite.

Avec letravail de Möruc (1902 sur les l'antoporles recneillis par la "Valdivia ", le catalogue de la fameantaretique s'accroit encore, non pourtant dans la mème proportion qu’à la suite des campagnes du "Cluallenger". M. Möbius établit le nouveau genre Leiomymphon (Ammothen) pour des formes essentiellement antaretipues, dont 11 . Pfefler avait déji signalé denx espèces, le $L$. gramle ot le L. l'lumsii, rangées par lui dans le genre Ammothea. Il convenail de distinguer ce genre des Adelin, mais non de l'éloigner de Ammothéides pour le mettre parmi les Nymphonides, avec lesquels il ne présente ancune affinité. M. Möbius n'attrilue à ce cornre. qu'mue seule espèce, le Leiomymphon (Ammothen) striatmm nov. recueilli par la "Valdivia" anx iles Bouvet, mais sa Colossendeis gibhosa nov. tronvée anx mèmes lienx, doit y prendre place, comme l'a montré plus tard M. Hodgson, qui a en outre identilié très justement eette espèee aver le Leiomymphon (Ammothru) grande Pfefler. Dans les panx sublittorales

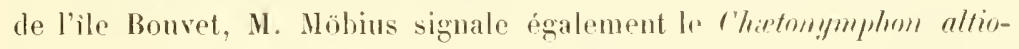
culatum nov. identique avec le 1 h. Mustrale Hodgson : Ins Pallenopsis glabra nov. et $P$. hrevidigituta nov. of la P'sendopallene comigera nov.; il retrouve aux Kerguelen quelques espèces déjà conmes : le Thatomymphon hrevicaudatum Miers, la C'olossendeis robusta Hoek et le 
Pycnogonum littorale Böhm, dont il fait à juste titre une espèce nouvelle, te $P$. magnimstir. Tontes ces espèces hahitent les eaux sublittorales; elles sont décrites et figurées avec soin.

Nous arrivons maintenant aux travaux exclusivement consacrés à la faune des régions antaretiques. M. Pfreller avait inanguré cette série que M. Hobrsux, gràce aux expéditions anglaises, va continuer d'une facon brillante.

La campagne du "Southern Cross" n'offrit pourtant à ce zoologiste fu'une seule espeice, le Vymphom anstrale nor. (1902, 23i), identifiée dans la suite avec le thotomympilhon altisculatum Möbius (Hodgson, 1907e) de la "Valdivia ".

Mais la Yrtional antaretic Erppetition de la "Diseovery "fournit à 11. Hongsox la matière d'une ample revanche (1907a). Les Pyenogonides recueillis au cours de eette campagne ne comptent pas moins de ringt-huit espèces, dont Loutes sont nouvelles à l'exception de cinq : la Pseudopallene romigera Möbius et la l'allemopsis ylalura Höbius découverte par la "Vatdivia " aux environs de l'île Bouvet, la Pallenopsis pilosa Hoek capturér par le "Challenger " dans les eaux subantaretiques de la province africaine, le Chretomymphom australe IIodgson ( $1 \%$. altioculatum Möbius) pris au cap Adare par te "Southern Cross " et à l'ile Bonvet par la "Valdivia ", et le Lcionympton Ammothen) grande Pfeffer déjà signalé dans la Gróorgie du Sud.

Parmi les captures faites au cours de l'expédition, la plus importante est, sans contredit, celle du I'entrmymphon antureticum nov. (1904, 1905 b. 397 ; 1907a, $36-39)$; la découverte de celte forme démontrait, avec une pleine évidence, non seulement yue le type décapode peut se réaliser chez les Pycnogonides, mais en outre qu'iln'y est pras une anomalie et qu'il peut apparaitre dans des groupes bien dillérentes: les Pentamymphon, en etfet, sont de vrais Nymphonides, alor's que les Decolopoda se rapprochent beancoup des Colossendeis. Il faut relever en outre dans le travail de II. Hodgson la description de deux genres nonveaux, Austradecus ef Austroiaptus, qui se rangent dans la famille des Ammothéides et semblent propres aux mers antaretiques, l'abondance et la variété dans ces mers des Leionymphon (Ammothea et des Chatonymphon, et la présence des 
Rhynchothoras, lormes aberrantes qui semblaient jusqu'alors propres à la Méditerranée.

- Les rícoltes de la "Discovery" ont étŕ faites en province africaine, dans la mer de lioss (baic Mac-Murdo), près de l'île Conlman, au large du cap Adare, du mont Terror et de la Barrière; elles sont toutes sublittorales, provenant pour la plupartde 8 à 180 brasses, rarement de 300 ou de :00 brasses. Les formes nonvelles qu'on y trouve sont les suivantes: Phoxichilus australis, Pulleropsis villosa et hirmalis, Charmymphon villosum, hiarticulatum, et mendasum; quatre especes de Nymphon hiemale,

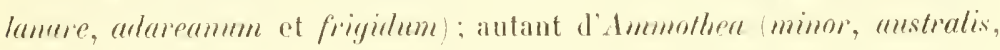
spinosa et glarialis); thois autres Ammothéides, l'Austroderus ylaciale, le

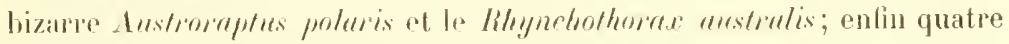
especes de Colossemle is (rugosa, frighidn, glucialis, amstrulis), les premières connues dans la rógionantaretique proprement dite, où elles trouventà de laibles profondeurs la basse températme que recherehent la phupart des représentants du genre.

A la mème éporjue, $\boldsymbol{H}$. Ilogason entreprenait l'étude des Pycnogonides recueillis dans la province de Magellan par l'expédition national antaretique écossaise. C'est aux Urcades que les naturalistes de l'expédition retrouvèrent la fameuse Decnlopoda austrulis, et c'est M. Hodgson qui eut l'heureuse fortune $\left.1905^{\mathrm{a}}, 1905^{\mathrm{c}}\right)$ non seulement de domner droit d'asile à cefte curieuse forme méconnue, mais encore de faire connaitre le travail oublié de J. Eights qui lui avail été signalé par 11. Calman (1). Les autres espèces antaretiques capturées par la "Scotia " (Hodgson, 1908 sont moins nombreuses que celles de la "Discovery ", et toutes différentes à l'exception du CWofomymphom mendosum et du Pentanymphon antarcticmm. Certaines étairnt déjà connues : le Nymphon compactum lloek capturé par le "Challenger" "aux environs d'Auckland; le Chrtonymphon brevicandatum Miers depuis longtemps connu aux Kerguelen, signalé par Pfeller à la Grorgie du Sul el retronvé aux Oreades; l'Ammothea (Achelia ronmm mox Bouvier, prise par le "Francais" en d'autres points de la province magellanique; deux Leionymphom (Ammothon), le

(1) Wusox $(1880$, 467) fait eutrer le genre Decolopoda dans son lableau systématique, mais il le caractérise seulement par ses chélicíres et ses palpes. 
grande et le Clausii signalés par Pfeffer à la Géorgie du sud et retrouvés aux Orcades, enfin la Colossendeis leptorhynchus Hoek, espèce abyssale prise par le "Challenger " dans la province africaine. Les espèces nouvelles sont les suivantes: la Pallenopsis lanalu des Orcades; deux espèces de Vrymphon, articulare et longicora, cette dernière espèce abyssale; denx thetonymphon, l'orcadense pris aux Orcades et aux Falklands, ef l'ussimile des Orcades, enfin une Colossendeis, l'orcadensis, également des Orcades. Toutes les espèces reeueillies furent capturées dans la zone sublittorale des Oreades ou des Falklands et sont, par eonséquent, subantarctiques; pourtant la récolte comprenait deux espèees abyssales, l'une franchement antarctique, le Vymphon longicoxa, et l'autre subantaretique, la Colossenteis leptorhyncha.

Entre temps, M. IIobcisox consaerait un mémoire (1907b) aux Pycnogonides reeneillis par l'rxpédition magellanique hambourgeoise. Ils eomprennent six esprices, dont trois nouvelles: Dymphon tridentatum, T'anystylum longicandatum et Coblassendeis putngonica; les autres sont: l'Austrolecus glaciale IJodgson trouvé plus au sud par la "Discovery ", l'Achelia Wilsoni Schimk, l'Ammother (Ammothella) Hoetii Pfeffer et l'Anoploductylus petinlutus Kröyer, une espèce que l'on eroyait jusqu'alors localisée dans les mers septentrionales, où elle fut décrite par Hodgson sous le nom de Pallene pygmaa et par Dohrn sous celui de Phoxirhilinlium longicolle. Dans ce travail, M. Hodgson identifie, peutêtre à tort, le genre Yoteniu Dohrn avec le genre Tanystylum fondé par Miers; il établit en outre la division, signalée plus haut, des régions antaretiques et subantaretiques en trois provinces: magellanique, ifricaine et australienne.

A l'époque où M. Ilodgsson publia ses premiers mémoires relatifs aux Pentanymphon, et aux Decolopoda, on ne croyait pas à l'existence de Pyenogonides à dix pattes, et M. Loman (1905), qui avait eu connaissance du travail d'lüghts sur les Decrloporla, pensait que l'établissement de ce genre reposait sur une erreur dans la numération des appendices.

Mais, après to travail de M. IIorlgson sur le Pentum!jmphon antareticum, il fallut bien se rendre à l'évidence, et M. CoLs, qui avait aussi retrouvé le travail d'Eights, publia un opuscule (1905) où il se livrait à des consi- 
dérations phylogénéliques fondés sur l'existence de Pyenogonides décapodes. Pour cet auteur, le gernere Derolopmele est lit forme acturllo la plus primitive du gromper des I'yenogonides, at l'on peut en faime driver deux séries divergentes qui constiturut lo zroupe : la premiones série,

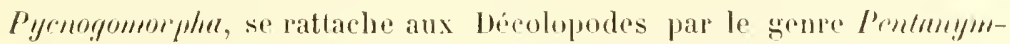
phom et aboutit aux Pycnogonidés en passant par los Nymphonidís, Pallénidés, Phoxichilididés et l'hoxichilidios; la seconde, Coloxsemelemmorpha, se rattache aux becolopodes par les Eurgeydidés ot aboutil aus Colossendéidés en passant far les Ammolhéidés. Les considérations d. M. Cole sont fort intéressantes et montrent bien limportance des précieuses découvertes elliectuées dans les mers antarefiturs; mais, s’il est juste de fecommaitere qur los Derolopurla sout les formes acturlles los plus primitives dreloudre dro l'yenogonides, il n'est pas possible d'y rattacher

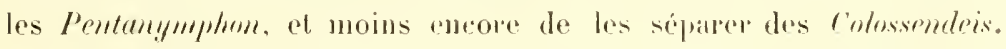
M. Ilodgson a tries habilement mis en lumiero $\left(1905^{\mathrm{c}}\right)$ les allinités choites

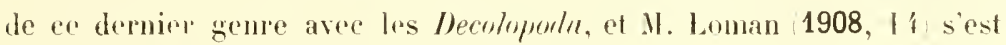
bevi contre l'áloignement des deux lormes.

Les études 1905, 1906a, 1906b) que jai consacrors amx l'yenogomides du "Francais" sont à peine postériemes anx premiones dr M. Hodgson. Il s'en lant que je los lrouve irréprochables; faute de matériaux de comparaison et sans toute aussi paree que je débutais daus le grompe, elles laissent id désirer au point de vue de la distinction des espreces el surchargent fachensement la synonymie : ainsi que lavait supposi

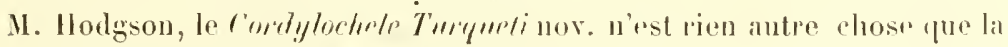

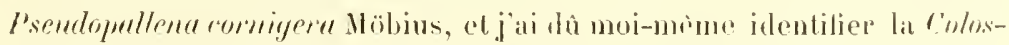

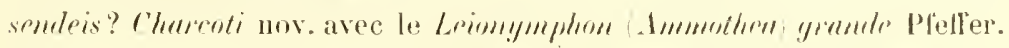

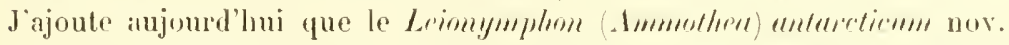

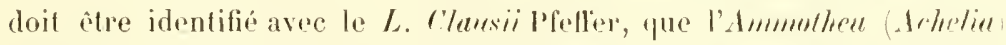

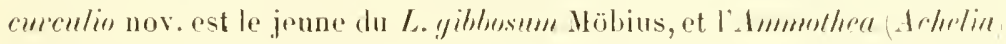

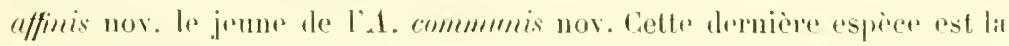
seule especr du "Frameais" qui soit vraiment nouvelle, avec une sreonde

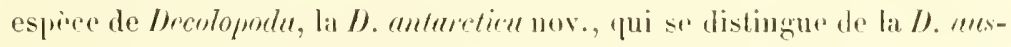
tralis far tivers caractires, entre autres, par ses palpes qui nont pur huil arlicles au lieu de nenf. Les récoltes du "Français " se bornent aux

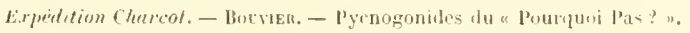


sept especes precédentes of au Pentrnymphlom antarticum llodgson ; le tout fut capturé en pleime région antaretique, dans la province de Mlagellan, a des profonden's qui atteignaient au plus 40 mètres.

Les considérations générales qui accompaguent ces études, surtout la drrnieve 1906b), me paraissent laisser beancoup moins à désirer. A l'rxpmple de M. Ray Lankester, je rattache lres Pyenogonides a la classe Are Arachnides el je les groupe en deux séries forohtives, les Colossendémorphes et les l'ycnogonomophes; res doux séries, toutefois, ne ressemblent ni à celles établies par M. Kay Lankester, ni à celles de II. Cole; la premire se limite anx Décolopodidés et aux Colossenthidiss; la seconde comprend tous les autres l'yenogonides, que je divise en Euchelutu, Cryptochelutu et Lehelutu suivant le systime de M. Sars. Les deux séries se rattachent à ume forme ancestrale hypothétique assez ana-

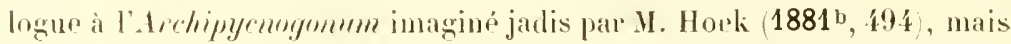
pourvu de cinq paires de pattes; les deux séries divergent a partir de la forme hypothétique et débutent liums el l'autre par un genre actuel déapode, la premiere par les Decoloprodu, qui, directement, conduisent anx

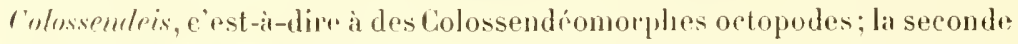
par les I'prthm!gmphom, auxquels font suite de nombreuses familles ortopodes, aver las l'yenogonidís comme famille terminale.

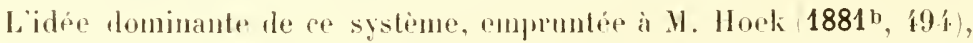
est le groupement des Pycnogonides rn séries crolutives, rhatue série ayant pour proint de départ une forme decapode considérée romme primordiale; cette idée me parat juste, mais le systime qui en dérive a dù subir des modifieations importantes a la suite des précieuses découvertes ellecluées par le "l'ourquoi las?".

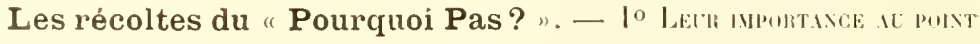

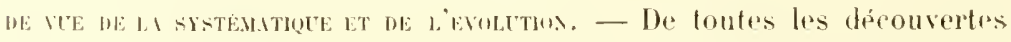
dont on est redevable an "l'ourquoi l’as?", la plus importante rot, sans contredit, celle d'un nouvean type décapodo que jai appelé P'entupyram 1910a, peur indigner à la lois sa structure el sa position zoologinpur. Las l'enlup!yrum, en elfot, sont des l'yenogonides typiques dont

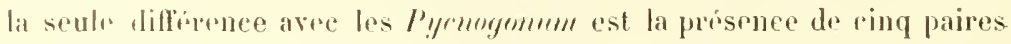
de pattes an lieu de quatre. 
Si, comme je tr pense, ef comme on le dimontrra plus loin p. 21, Jus formes déeapodes doivent itre consideries eommo primitives, il est impossible de placer la famille des l’yenogonidés au sommet de la síne des Nymphonomorphes, à la suite des Ploxichilidés; les formes octoprodes, e'est-à-dire dérivées, me sumaient dommer naissance à des formes primitives ou dreapodes. An surplus, comme la justement observó 1I. Carpenter 1894, I98) ot it sa suite 1I. Loman (1988, 9), fos I’yemegonidés ont unestructure spéciale fort diffépente de colle dres Pboxichilidés, de sorte quils ont atteint lour btat de degradation actuel par me voir plyylogénélique indipendante. Nous sommes done conduits ì établir pour

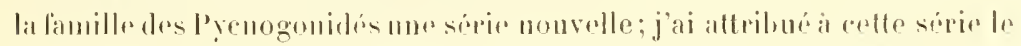

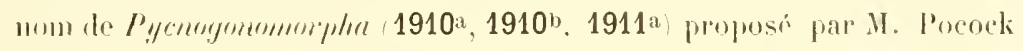

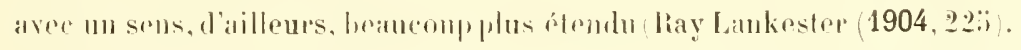

Nous voici fone en présence de trois séries devolutives qui comprennent

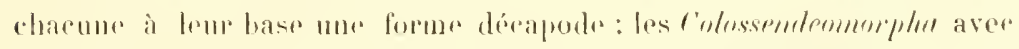

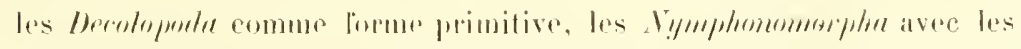

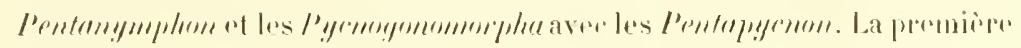

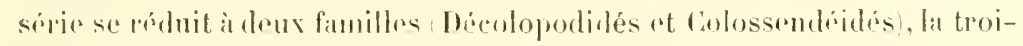

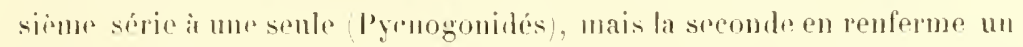
gramd nomber qui smmblent évolues snivant deux directions dillé-

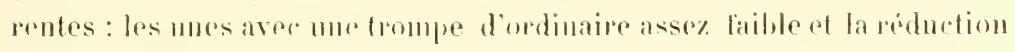

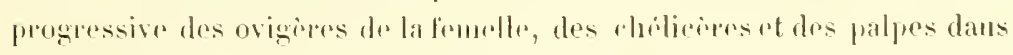
les demx sexes, - los autres aver une trompe toujours volmminense,

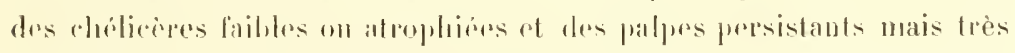
variables. Le premior groupe est pomatruable patr ses enchainements :

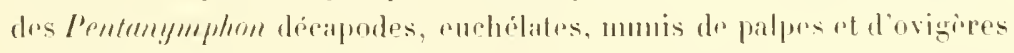
bien developpes, voire de formes phus prinitives eneore, il conduit. d'une part aux Nymphonielés ortopodes et anx Pallinides ou les palpes siatro-

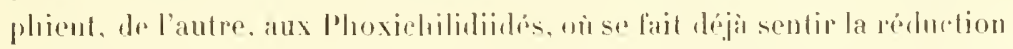
des ovigines de la femelle, el anx lPhoxichilidis ou dispamaissent totalement palpes ef rhéliripes dans les dems sexps, en mine temps que fos ovigeres dans la lemella. Le premier gronpe constilue la série des. Vymphlu-

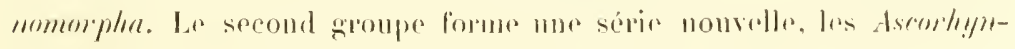

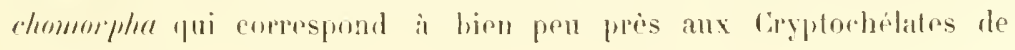


II. Sin's; il se linite des lor's anx buryeydidis, lont les ovigeres ont une griffe terminale ef les patpes dix articles, ot aux Ammothédés. qui sont moins prinitifs papere gur frurs palpes comptent moius de dix

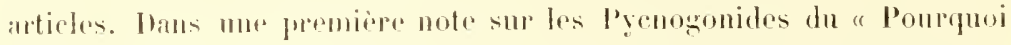
l'as? " 1910a, 30/, javais rangé les Ammothrides avec les Nymphonomorphes: on verra dans la suite pourquoi il comvient de leur domer une place a la suite des Euryeydidis.

Ainsi, d'apres les découvertes du "lourpuoi las? " les l'ycmegmielesse divisent ent qurtere séries érolutires caractérisés chacume par des traits morphologingues spécianx : les Colossendéomorphes, par leur srande taille, lours oriliens sexuck représentés it la base de tontes les pattes, la contisuitté de leurs palpeset dre lenrs ovigreses les Nymphonomorphes, par leur trompe ordinairement réduitr et latmphie progressive de fous leurs appendiees cophaliques; les Ascorhyuchomorphes, par leur trompe volu-

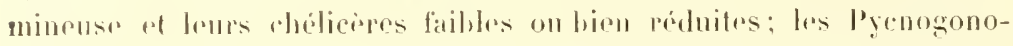
mopplers, par lour corps condensé, leurs pattes courtes, leurs orilicass

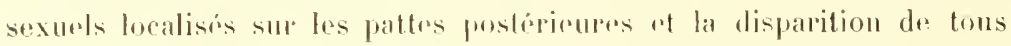
les appendices eriphaliques à l'exception dres ovigines du màle. Cees quatere séries présentent toutes à leur base une forme décapode, sauf Ioutefois redte des Ascolhỵchomorphes, qui me parait moins fortement itablir que les autres, paree qu'on n'y combait pas eneore de formes à dix pattres. Il uost pas impossible gur les Ascorhynchomorphes se rattachent, comme los Phoxichilididés, à des Nymphonomorphesplusprimitifs

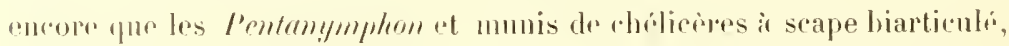
ave des palpes de dix anticles; mais refte supposition ne repose sur aucun lait frécis, et to groupe diflère a tel point des Nymphonomorphes qu'il partait plus juste de le considérer comme représentant à lui senl ume série independante. La question n'est fas résolne; ello sera tranchée si l'on trouve quelques jours mo Aseorhynchomorphe décapode.

1) noi qüil en soit, ces quatre sirries sont fort dillérentes les unes des autres u, comme trois d'entre elles nons oflient encore des représentants de lane itat primitif dicapode, on pent en conclure que les l'yrnogu-

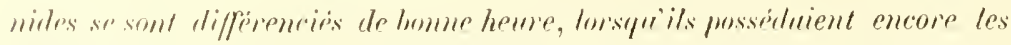
dix puttes de lo forme ancestrato 1910a, 30). Cotte conclusion interessante 
de mente que lat division des Pyonogonides en qualder séries frolutives

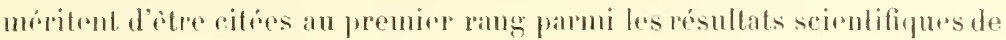
la campragnedu " l'ompuoi l’as?" du moins relativement am groupe qui Holls oceuper.

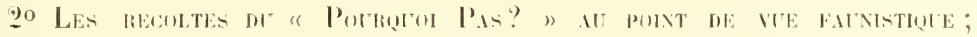

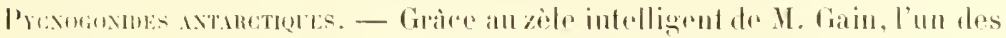
nalumalistes de l'expédilion, la campagne du a Pourquoi l'as? n fut singulirrement fructuruse; coll ce qui concrene les l'yenogonisles, elle se place sur lomène rang que la plus léromde des campagnes antareliques, colledr.

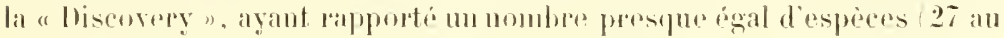

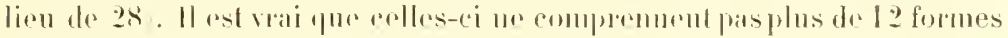
momrelles, tandis qu'on en rompte 23 dams les résoltes do la " lisco-

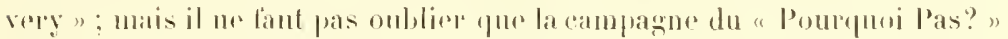
est la dermiere en date, de sorte quidle devait forerment capturer bean-

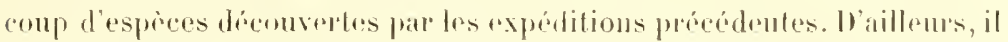
n'ilat pas saus intepet, au point de vur launistique, deretrouver daus la

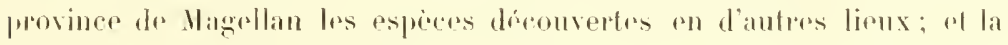

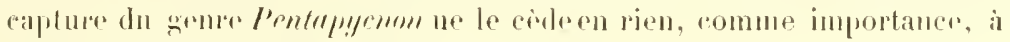
collidu geure l'enlan!ymphom.

Je crois utile do pelever ici un comple rendu tris sureinct que jai

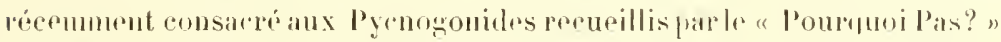
1911a); recompte rendu met rolumiore l'importance lannistique des captures laites an comes de lexpridition 1 .

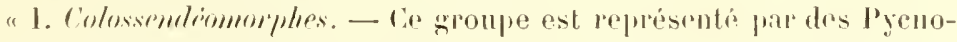
gonides ordinairenent voluminemx qui recherchent presifue tous les eaux froides, de sorte quils sont alygsarux dans les rogions tropicales on tropépés at ne pemontent an voisinago du littoral que dans less régions polajess. Le grompre compremd dems familles : los fócolopodidís et les cotossentéidés.

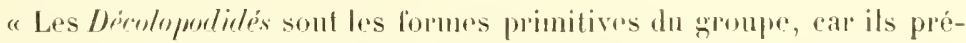
sentent encore des chélicios birn diveloppées at conptront cint paires de paltes. La famille se réduil à deux ropieces, l’une découverte par lo

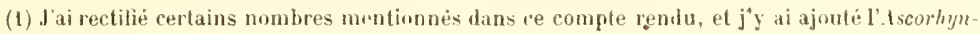
chus glaber lloek, It: seul Eurycydidé propre aux mers antaretiques. 
"Frangais" D. anturetirn Bour., l'autre II. anctolis Eights. ancienne-

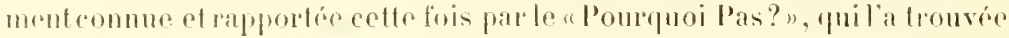
aux Sloctands. Cette soeonde espece parait moins franchement antaretique que la promière.

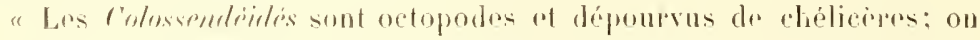

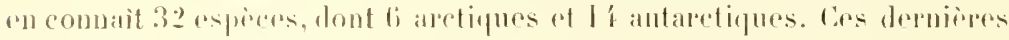

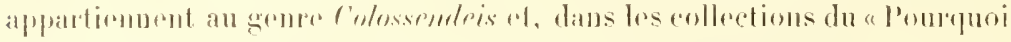

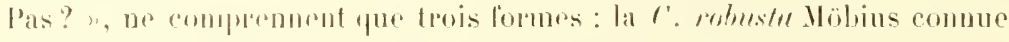

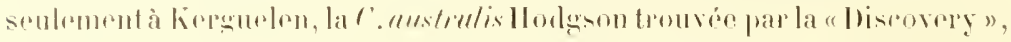

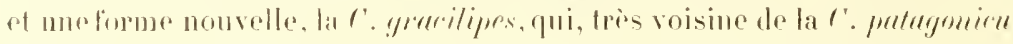
Irudgson, s'en dislingue par divers earactires, mais surtout par son

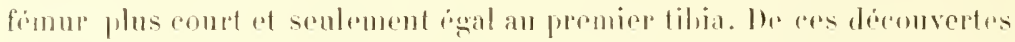

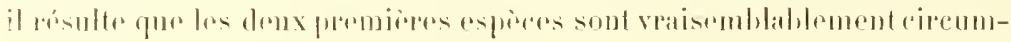

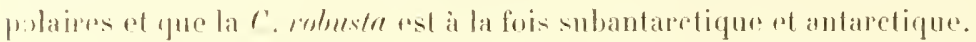

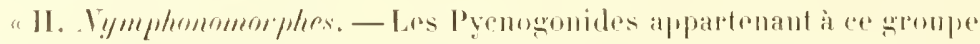

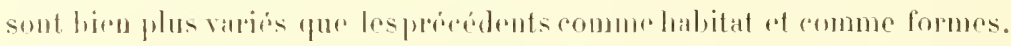

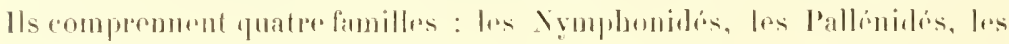
Phoxichilidialés el les Phoxichilidés.

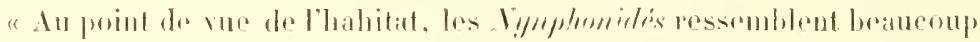

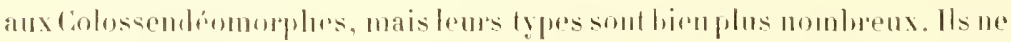

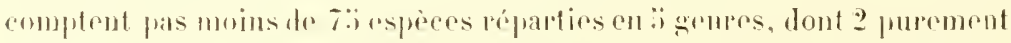

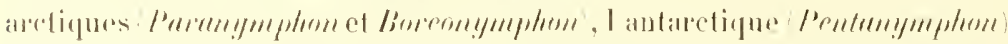

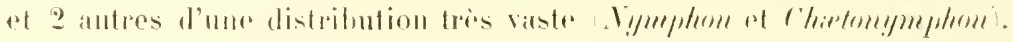

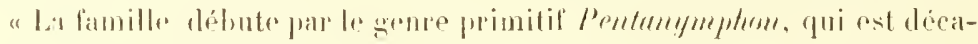

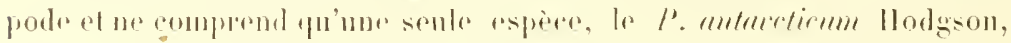

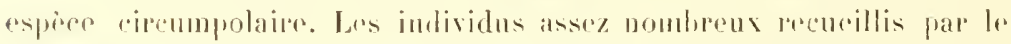

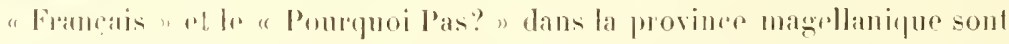

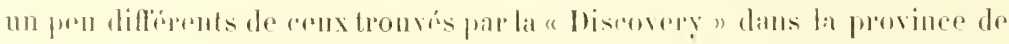

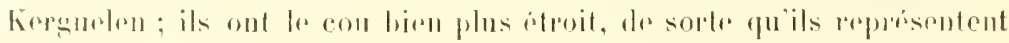
peut-iter more forme focale.

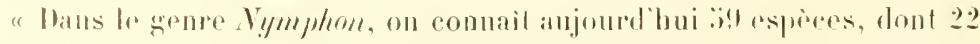

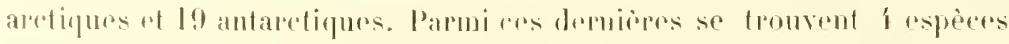

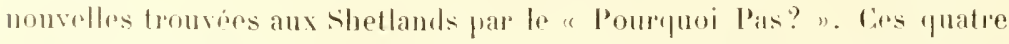

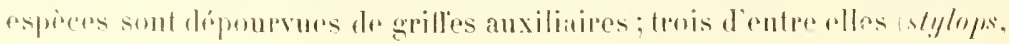


Gharerti, temeipes se rangent parmi les Nymphoms ou le premier tibia n'est pas sensiblement phus long on reste mene plus romet que le srecont; la quatriome espece frocerwiles appartient an groupe où be serond libia

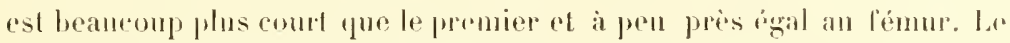
1. stylops possederencore des moliments de grifles ansiliaires; il a le trone

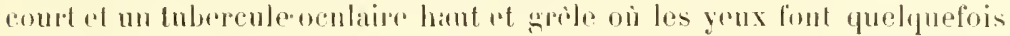
défaut; ést une respecepullulanle, à en juger par les nombremx exemplairescapturés d'un stul coup; il se rapproche d'une espose d'Auckland.

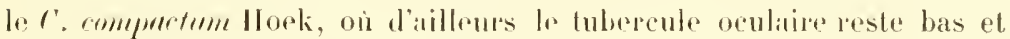

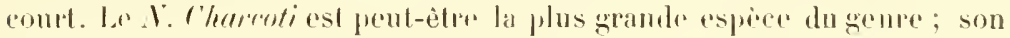
tubereule oculaipe ast an dome, et som fémme ézale le premiop libia;

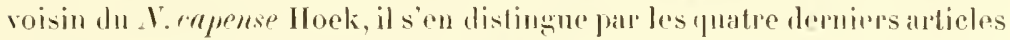
subegand de ses palpes. Le $X$. trmipes, aimsi nommé à canse de ses pattes greles, est catractérise par la longuenr pródominante du deuxiome article deses palpes, on quoi il ressomble it drux rsperes chilirmues, lo

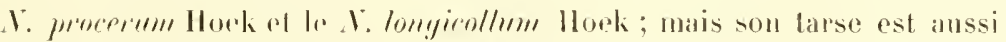
long yue le proprode, lamdis qüil ast plus cour dans les deus formes

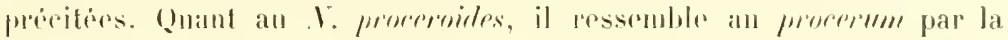
forte dilatation terminale do la demsiomo cosal cluez les femolles, ere qui

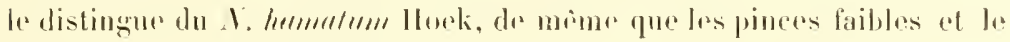
tarse plus eourt que le propude.

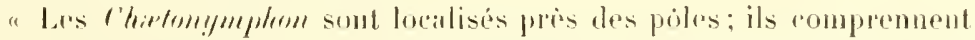

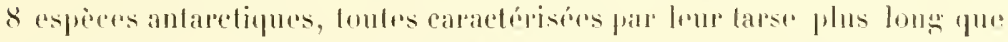

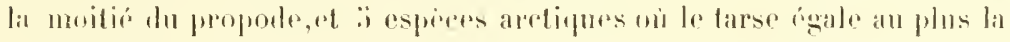

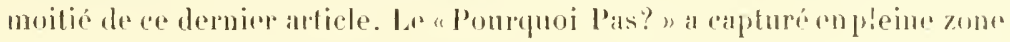

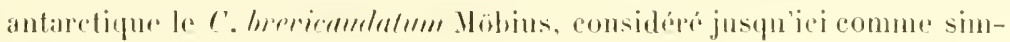
plement subantaretipue.

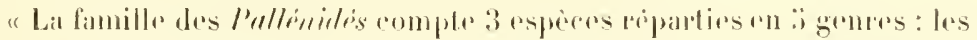
Pallene, rípaudus partout et ordinairement sublittoraux; les I'mrapalleme,

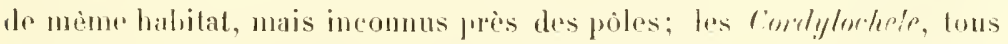

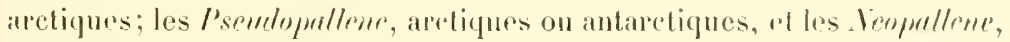
¡ni habitent la region miditeranfenme. Les Pallonides se maltachent étroilement anx Tymphoniles par les /'urapalleme; eomme l'a établi böhm, et comme jai pu men convainere en etudiant les récoltes de M. Marmund 
au Japon, le I'. Iongierp. Böhn a des palpe's aussi longs que la trompe, mais réduits à deux articles, dont un hasilaire fort petit.

"Les especes recueillies par le "Punrpuoi l'as?" se rangont dans le genre Psemlopulleme, qui compte quatre représentants antarctiques. Ces espirces sont an nombe de trois: la $P$. romigem Möbius Fordylochele

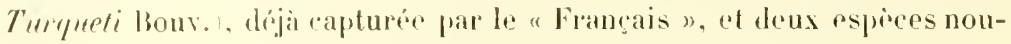

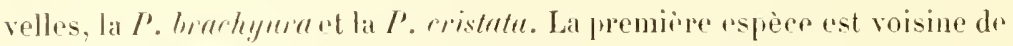
l'unstralis ILodgson, dont elle se distingur par son abdomen róduit à une légere saillie vertirale ot par ses pinces on le doigt lixe est largement obtus; la seconde ost ribhement arméc d'épines, comme une espece are-

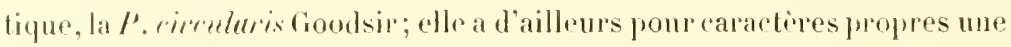
tromper effilo, des pinces a doigts inermes ot de hautes saillies dorsales.

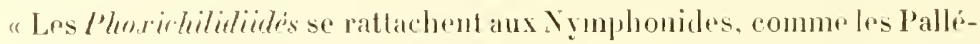
nides, dont ils difterent par leur tubereule optique situé tris en avant. Leurs esprices sont au nombre dr 49, distribuces on 3 germes : z localisés daus la zone sublittorale des rígions chaudes ou tempérées (Rigomm, Mnlosomme et 3 autres qui ant une distribution plus large: Phoridrildium avere 2 especes qui sont propres a lhémisphere horéal: Amplotartylus avec 19 especesdont 2 subaretiquesot 2 subanbretinues; entin Pullemopis, qui comple rgalement 20 espirees, dont 2 arefigues ot 7 antaretiques. Trois deres dernieres ont itcipportés par M. Chareot: P'. pilosu Hork, recueillip d'abord par le "thallenger p el retrouve par la "Discovery"; I'. gluturu Möhius, également eapturée par la "Discovery" "t decouverte par la "Valdivia " enfin ume lomene nouvelle, la P. macenmyr, aiusi nommée à eause de sa grifle trominale à pruprès aussi longue que le propode; a ce proint de vue, notro esprece ressemblo à la fl. loreridigituth Mähius, dont colle se distingue d'ailleurspartous les autres carar-leres, notamment par son rorps discoüde ot par ses pattes courtes, wì le fémur niest pas phus longe que le céplablothomax.

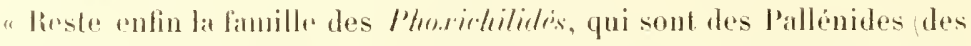
Phoxichilidides plutrit ou les chélicères ont dispraru de mêmr que les rudiments de palpes. La fanille ne renterme gu'un genre, I'bur irtritus, avee 7 esprees litlorales ou sublittorales, dont une aretipue et une antaretipur. Cotte dernicre est le l'. arestrulis llodgson, dont on ne con- 
naissait qu'un spécinen capturé par la " Diseovery " ; le " Pourquoi l'as?" en a recueilli plusieurs individus sur lespuels jaipu examiner les orifices des très nombreuses glandes rémentaires; le diamitre de res orifices nexecde pas $\$ 0$ \%

"III. Ascombynehomonphes. - Les formes décapodes ne sont pas encore conmues dans ce groupe qui romprend deux fanilles, les Euryogdidés el les Ammolheidrs, celtr dernire justenent divisée par M. Loman en Nymphopsinés et Ammothéinés.

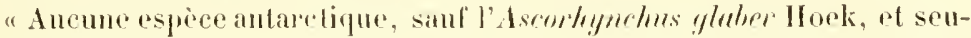
lement í aretiques parmi les 2' qui eomposent la lamillo dos Euryrydirlos; aurune espece polaire parmi les 10 Nymphopsines acturllement eonnus, abors que l'on trouve 17 espreses arcliques et 18 antaretiques parmi les :36 espiees de la sous- famille des Ammothéinós. Les 18 esporces d'Ammolhéinás antaurtiques appartiennent à 7 gemes, parmi losquels 3 seulement, Leimymphon, Ammothen et Anstrmaptus, sont représentés dans les rícolles du "l'ourquoi l'as?"

"Le genre Leionymphon (Ammothen) compte fll especes toules antarc-

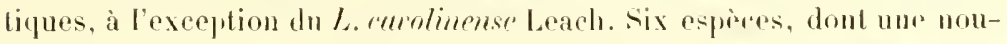
velle, ont été recueillios au comrs de l'expódition: lo L. striatum Möbius et le L. gibbosmm Möbius, conmus semlement à l ile Bouvet où ils furent rapturés par la "Valdivia "; le L. grumle l'feller et le L. C'lamiil P'feller signalés à la Grórgie du sud; le L. mimes llodgson, qui est nue espèce circumpolaire, ear on le retrouve an sandwich du sud (exemplaim communiqué par $\mathbf{1 1}$. Lahille) et dans les parages plus antaretiques visités au cours de l'expédition; enfin une forme nouvelle, le L. grarilipes, qui se distingue du L. minus par so's pattes bien plus grèles ol par sil deuxieme coxa bienplus allongre. Ces malériaux très riches unontpernis

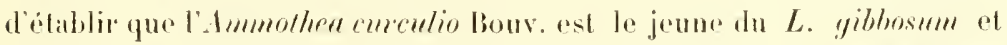
qu'il comvient d'identilier, comme le pensait Ml. Hodggson, le L. antareticnm lions. avee le L. rlamsii.

"Les Ammothen Achelin antarctipuesse limilentà trois espèees, car l'A. Ilumbiil'feller est sùrement une Ammothrllı. Parmi ces trois espèces, deux seulement sont bien connues el proviennent dos campagnesfrancaises. L'une est l'A. commmumis Bouv., espèce pullulante dans la province magellanipue

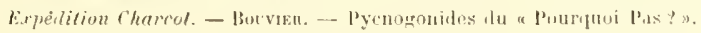


où elle fut découverte par le Francais, puis retrouvée par le "lourquoi Pas? ", et une espècenouvelle que jappellerai A. serratipalpis à cause de ses palpes dont les articles 7,8 et 9 sedilatent inférieurement et, par leur ensemble, forment une sorte de scie. La mème disposition s'observe dans 1. Leionymphon minus et le L. gracilipes; an surphus, l'espèce nouvelle est la plus primitive du genre, ear son eorps est peu condensé et nettemeut articulé: son armature épineuse est réduite au minimum et ses pattes sont notablement allongées.

"Je signale, pour terminer, une espèce rarissime, le singulier Austroraptus glaciale Ilodgson, dont le "Pourquoi Pas? a capturé d’intéressants exemplaires.

"W. I'ycnogunomorphes. - Ce groupe est représenté par l!' espèces réparties près du littoral ou à de faibles profondeurs. Il ne comprend qu'une seule famille avee les deux genres l'entrpycnom et Pycnogonum, étudiés dans des Notes antérirures. Le genre P'entupyenon est décapode et se place à la base du groupe; il est représenté par deux espéces : l'une antaretifue, le P. C'Lmeroti Bouv., l'autre de la Guyane où elle fut trouvér par Geay ( $I^{\prime}$. Geayi Bouv.). La découverte des Pentapyonom est certainement l'une des plus belles et des plus suggestives parmi eelles dont nous sommes redevables aux naturalistes du "Pourquoi Pas?" On silit que les mêmes naturalistes ont eapturé un rrai I'yrnogonum, le P'. Gaini, dans les eaux antarctiques. "

En résumé, l'étude des Pyenogonides reeucillis par le "Pourquoi l'as?" conduit à cette conclusion que la fumme du groupe est très richedans les régions anturcliques, boaucomp phus riche certumementquedans les régions arotiques. Cietterichesse prédoninan te est un fitit aequ is désorma is : avantla campagne on connaissait 68 es rices de Pyenogonides antaretiques et 62 autour de l'autre pôle ; aujourd'lui la dithŕrence est phus grande encore; les Pyenogonides des mers froides de la région australe sont au nombre dr 82 espéces, alors que cenx des mers aretiques restent au chiflre ci-dessus tixé.

llais, comme je l'ai fait observer (1910c, 11 hl), " ce n’est là qu'uu début; explorérs depuis très longtemps avec beareoup de soin par les chereheurs des deux mondes, les régions boréales ne nous réservent plus guire de surprises, tandis que les campannes antaretiques, peu nom- 
breuses encore, rapportent toutes des trésors insomponnós. Lelle du "Pourquoi Pas? ", la derniope en date, ne nous donne-t-elle fas 12 formes nouvelles sur $28 \mathrm{el}$, dans ce nombre, le type des Penloyyrnom, qui justitie les modifications profondes introduites daus la phỵlogrinie et le classement des Pyenogonides? Afin de montrer justu’à quel point on feut compter sur les tampagnes antaretipues pour étendre nos connaissances zoologiques, il me sulira de rapporter le fait suivant. Hans la baie de l'Amirauté, aus shetlands, par t20 mètres de profonderr, un seul conp de chalul a rapporté las a especes nouvelles de Nymphore, le

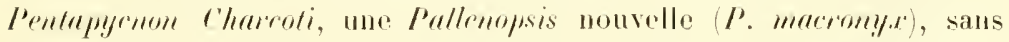

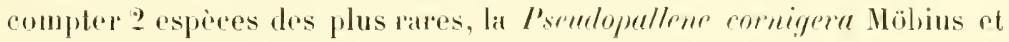

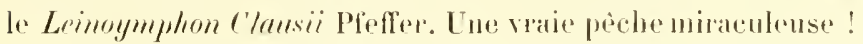

"Cetfe richesse de la fanno antarelique n"est rertainement pas propre aux Pyrnogonides; elle doits'étendre à d'autres groupes. H convient de l'attribuer, ce me semble, à la présence d'un continent polaire dont les rives of les illes avoisinantes héberogenl d'abondantes Diatonté of mne végétation sous-marino où peuvent trouver un aliment toutes les espèces, quel que soit leur régime. "

La liste des esprees antarctiques et subantaretiques a cht soigneusement établie par M. Horgson (1912, 162). Jo roleve cette liste en la complétant avee les espèces recucillies au cours de la campagne du "Pourquoi l'as?" (ces espèces sont indiquées par un astérisque) et en y établissant les divisions systématiques adoptées dans l'ouvrage (r. 20$)$.

Sur la signification des Pycnogonides décapodes. - Les Pyenogonides sont normalement pourvus de huit pattes. Malgré la découverte l'unc espece th genre Decolopoda par Eights, en 1834, c'est M. Hodgson qui a eu le mérite de faire connaitre l'existrneo dans le groupe te formes décapodes: il a exhuné (1905a), pour aninsi dire, le mémoire de . Eighls ef soigneusement décril cette espèce quela "Scolia " redécouvrit aux Orcades; il a enoutre signalé an monde scientifique le corieux I'enlamymphon unlatcticmm trouvé dans les mers antaretiques for la "Discovery" (1904). Après ces travaux développés dans des mémoires importants $(1906,1907)$ el justifiés par les découvertes de M. Jean Charcot, on 


\begin{tabular}{|c|c|c|c|c|c|}
\hline & 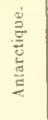 & 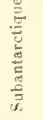 & & لَّ & 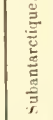 \\
\hline 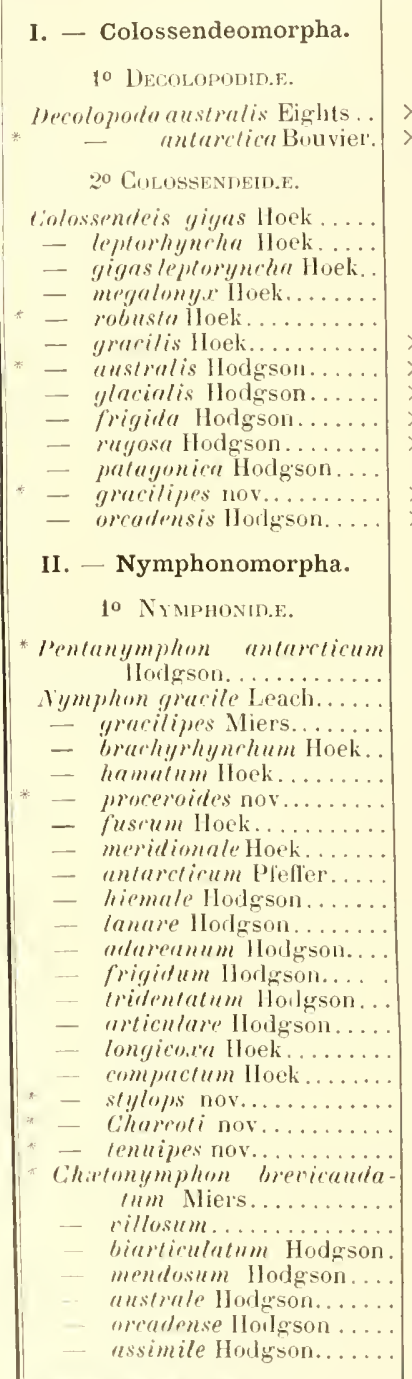 & \begin{tabular}{|c|}
$x$ \\
$x$ \\
$x$ \\
$x$ \\
$x$ \\
$x$ \\
$x$ \\
$x$ \\
$x$ \\
$x$ \\
$x$ \\
$x$ \\
$x$ \\
$x$ \\
$x$ \\
$x$ \\
$x$ \\
$x$ \\
$x$
\end{tabular} & \begin{tabular}{l||} 
\\
\\
\\
$x$ \\
$x$ \\
$x$ \\
$x$ \\
$x$ \\
$x$
\end{tabular} & 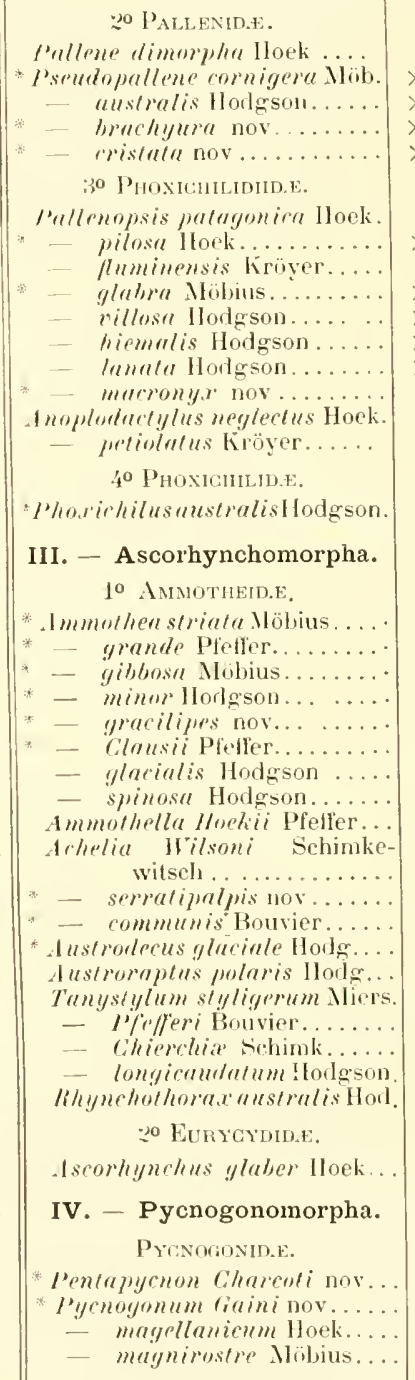 & \begin{tabular}{|c}
$x$ \\
$x$ \\
$x$ \\
$x$ \\
$x$ \\
$x$ \\
$x$ \\
$x$ \\
\\
$x$ \\
$x$ \\
$x$ \\
$x$ \\
\\
\\
$x$ \\
\\
$x$ \\
$x$ \\
$x$ \\
\\
\\
\\
\\
\\
\end{tabular} & $\begin{array}{l}x \\
x \\
x \\
x \\
x\end{array}$ \\
\hline
\end{tabular}


ne pouvait plus douter, comme avant, de l'existence de Pyenogonides à dix prattes.

Je ne crois pas que M. Ilodgrson ait imis une opinion au sujet de ces formes "xtraordimairement curieuses, mais d'autres zoologistes se sont montrés moins discrets. M. Cole 1905), on l'a vuplus baut, considère les Decolopodre comme nue forme ancestralr persistante d'ou sont issus, d'un còtó les Euryeydides, Ammothrides et Colossendéides, qui consti-

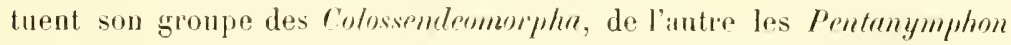
qui, arec toutes les antres familles, formentle groupe des I'ycnogomomorpha. de crois bien yu’à l'heure artuplle, el smrtout depuis la découverte des Pentapycnon, le savant zoologiste amóricain, anynel on doit de si bonmes itudes sur les Pycnogonides, serait le premier à introduire des modifications dans lo précedent systeune. Comme l'a justement écrit M. Loman (1908, 15), " il n'rst pas possible d'rlablir de parenté entre Pentamymphon et Decoloporla ", le premier genre ayant une segmentalion tris nette qui fait défaut an second, et d'ailleurs, dans toutes les parties de son corps et de ses appendices, une structure absolument differente. Cie sont deux formes primitives, qui ne peuvent dériver l'une de l'autre, rncore que la seconde soit plus ancestrale quo la premiere, car ello est moins différncién dans ses ovigries comme dans ses paltes; le scape de ses chrliceres compte cncore deux articles el ses palpes sont complexes.

Malerré ces observations, il faut reconmailre que M. Cole, avant tout autre, a considéré comme un caractere essentiel la présence de ciny paires de pattes chez les Pycnogronides.

Cetteopinion ne parait absolument justifiée, et jel'ai soutenueà diverses reprises, dabort dans mon étude sur les lyenogonides du "Français" $\left(1906^{b}\right.$, puis en laisant connaitre le genre l'entry"yrnon découvert par l. "Pourcuoi l'as?" (1910a et le Pentrylyrmon Genyi de la Guyane (1911a), enfin récemment dans une note présentéc au Congrès intermational d'entomologie $\left.1911^{\mathrm{c}}\right)$.

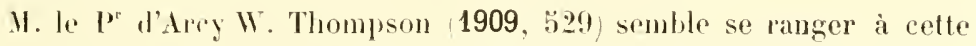
maniore de voir el, en loul cas, considipe les lecoloporla, sinon les autres formes décapodes, comme ayant un caractice ancestral. Par contre, M. Carpenter. dplus recemment M. Calman, emeltent mo opinion 
contraire et regardent les genres décapodes comme issus des gemres octopodes. A vrai dire, M. Carpenter (1905) n'est pas affirmatif, car il dit simplement que " peut-être, la cinquirme paire de pattes de ces genres représente une acquisition relativement réente " the fith pair of legs in these genera may possibly represent a comparatively new devolopment, par suite une açuisition quauraicut laite, indépendamment, les Decolopodu et lenturymphom, les seuls genres décapodes connus à l'époque où M. Carpenter écrivit son mémoire.

I. Calman s'est prononcé nettement en laveur de l'hypothèse précédente. "On doit admettre, écrit-il (1909), que la constance du nombre des somites et des appendices an milien des variations de structure relativement étendues présentées par les Pycnogonides à huit pattes suggère fortement lidée que ce plan de structure est primitil, c'est-ídire "normal " dans le groupe; par contre, le fait que la condition décapode se présente dans deux genres ayant peu de traits communs permet de considérer celle-ci comme une modification secondaire du plan primitif. " 11 y aurait beaucoup à dire sur ces assertions : la structure octopode est, de toute évidence, l'état normal des P'ycnogonides, mais il n'en résulte pas qu'elle représente l'élat primitif du groupe; comme la structure normale, e'est-à-dire actuelle, des autres groupes zoologiques, elle résulte des modifications d'une structure primitive, dont certaines formes (les Pycnogonides décapodes dans le cas qui nous occupe) ont conservé des traces. A l'appui de sa thèse, M. Calman cite l'exemple des Polyartemia, qui, par un accroissement métamérique secondaire, possèdent 19 paires de pattes prégénitales, alors que les autros Phyllopodes anostracés, plus primitifs d'après lui, n'en possident que 11 ; mais cette opinion est manifestement contredite par M. E. Daday dans sa belle monographie du groupe $(1)$ : l'ancêtre des Anostracés, observe cet auteur, " avait certainement phos de l! paires, et ce nombre ne s'est réduit que plus tard au nombre actuel... Les Polyartemia ayant 19 paires de pattes ef les Polyartemiella 17, ces formes ont. à mon avis, mieux conservé le type originel que les genres à 11 paires de pattes ".

(1) E. Damb De Ders, Monographie systématique des Phyllopodes anostracés (Ann. de's Sic. Mat., (Lool.), t. Xl, [. 411, 412, 1910\%. 
On peut en dire autant des P’yenogonides décapodes relativement aux Pycnogonides octopodes; toutefois, il ne convient pas d'étendre à un groupe les considérations phylogénétiques applicables à un autre; l'exemple tes Myriapodes, considéré au point de vue du nombre des segments, nous en donne la preuve.

Dans un article récent, suseité par ma note préliminaire sur les Pycnogonides du "l'ourquoi Pas?", II. Calman (1910) observe que la découverte du genre Pentupyrnon dans les mers antarctiques fortifie beaueoup l'hypothèse qu il a soutenue ì la suite de M. Carpenter : $1^{\circ}$ paree que les Pyenogonides décapodes sont tous loealisés dans les mers liroides australes ot qu'on ne saurait admettre que les Pyenogonides ont fait exclusivement leur évolution dans ces mers; $2{ }^{\circ}$ parce que le genre l'ycnogomum apparait comme très spéeialisé et que le genre Pentapycnon présente une structure identique, sanf la paire de pattes supplémentaires. Sur ee dernier point, M. Calman ajoute très justement que la présence d'orifices sexuels sur d'autres patles que les dernières, si elle était réellement constatée dans le genre Pentapycnon, serait de nature à infirmer son hypothèse et à justifier la mienne.

Je tiens à reconnaitre, tout d'abord, que ee dernier caractìre fait défaut: les orifices sexuels, dans le genre Pentrpycnon, se trouvent localisés sur les pattes de la dernière paire, comme dans les Pycnogomun, et ceux que j'avais cru pouvoir annoneer, dans ma note préliminaire (1910a, 28), sur les pattes des antres paires sont tout simplement des orifices de glandes coxales, comme il y en a chez la plupart des Pyenogonides, sinon ehez tous, sur la face dorsale de la deuxième eoxa. Ainsi, les Pentapycnon se distinguent seulement des Pycnogonmm par la présence d'une paire de pattes supplémentaires, et 1 . Calman peut utiliser ce fait bien établi, s'il lui trouve de la valeur. Par eontre, il ne saurait maintenir sa première objection depuis que j'ai finit eomnaitre le Pentupyenon Geayi (1911a, 491), une espéee fécapode abondante en Guyane; il n'est plus exact de penser, comme je le eroyais moi-mème, que les l'yenogonides à dix pattes sont propres anx mers froides australes; $c$ roiei un qui habite le littoral des tropiques où les eaux sont attiédies par le soleit, si bien que la forme décapode nous apparait indépendante du climat et des lieux. 
Mais c'est ailleurs quil fuutchercher la preme du caractìre ancestral des Pyenogonides i dix pattes. On ne saurait nier que les genres Hecolopodu et Colossendeis présentent des affinités lort étroites et dérivent sùrement l'un de l'autre; il esl de toute évidence aussi que la présence des chélicères est un caractire primitif. Or les ehéliceres sont hès développées et présentent mème un scape à deux articles dans les Decolopoda, tandis ju'clles font totalement défaut chez les C'olossemleis. Alstraction faite dn nombre des jattes, ce caractere est le seul qui distingue los deux genres, de sorte quil ast improssible de dire proe les lfecolopoda sont des f'olossendeis ayant acquis récemment une paire de pattes supplémentaire, et, par un atavisme singulier, la paire de chélicères que leurs progéniteur's avaient predue. Ne'est-il pas plus simplect parfitement logique d'admettre que les Colossendeis dérivent du geme Deeolopoda par la perte de deux formations primitives, les chélicires et les pattes de la paire postericure? Ainsi les Colossendéomorphes à dix pattes sont primitifs par rapport ì cenx ou les pattes sont au nombre de huit, et il n'y a amrmne raison pour 114 pas étendre la meme rigle aux Pyenogonomorphes, encore que les types décapodes, dans ces denx groupres, ne présentent ancun caractere primitif sprecial an dehors tu nombre de leurs pattes.

Au surplus si, comme le pensent 11 . Carpenter et ll. Calman, la paire de pattes postérirures est une paire surajoutio daus les types décapodes, les orilices sexuels des Pentupycnom devraint se trouver à la mème place que chez les Pygrogomum, à savoir sur les pattes de la quatriemm paire, alors qu'ils sont sitnés sur la cinquieme. Je crois d’aillemes avoir la preuve que la paire de pattes dont les types octoprodes sonl dépourvens niest point la derniere des types octopedes, mais la prócédente on avant-derniere. Comme on le verra phus loin ip. l:36 et l:ig), quand on compare le

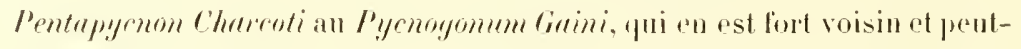
itre en dérive, on constate que les tubercules segmentaires dorsaux sont 'n mème nombre daus les deux espèces; le tuliereule dorsal prostérieur, celui qui correspond a la quatriome paire de patles dn l'entapyereon l'harroti, est enere parfaitement développé dans le Pyenongmum Cieanji, ou d'ailleurs la praire de pattes correspondante a disprarn. On constate lir

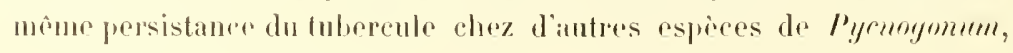


notamment dans los deux rspeers commmes de nos mers, lr l'. rmsirimostre et le $P$. littruale Strom.

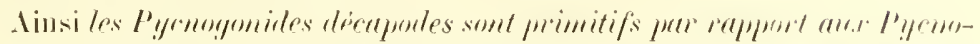

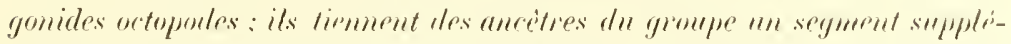

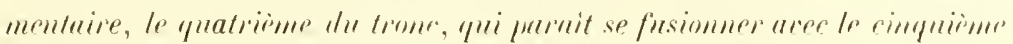
et peral sess appendires dums les esyeress melupundes.

Il est probable que les lormes primordiales de l'ordre possedaient plus de cinq paires de pattes, et loot possible, comme je jai dit aillems $\left(1906^{b}, \ddot{3}, 13\right)$, que de telles formes aientencore des représentants dans les mers acturlles. En tont eas, clles etairnt pour le moins décapoles, aver

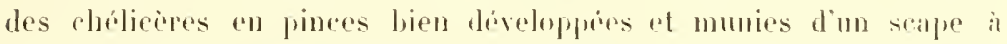
deux articles, des palpes richrment artieules, des orilices sevurels ut des glandes cosales sur la denxieme cosa de tontes los patles, un alulomen

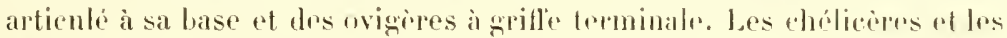
palpes des lyenogonides rorrespomblent aux appendices de meme nom des

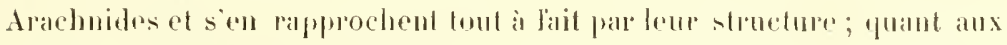

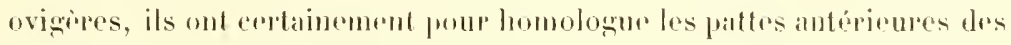
aninaux de cette dermiere classe. Comme l'ont observé plusients zoologistes (je citerai entro antres N. Ilock at M. Luman), les ovigemesont etro

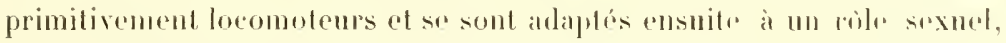

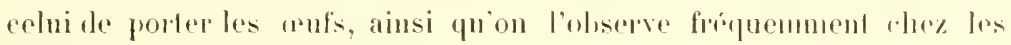

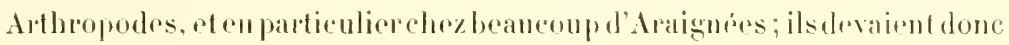
avoirla minestructurequeles pattes el prosenter, rommo ollus, mut grillo terminale. En linit, cette grifle as presente chez tous les l'yrnogunides primitilis, et si, chez ces dernirrs. on trouve aux ovigipres deux articles de plus que dans les pattes ( 10 au lieu de 8 , il faut salls doute aftributer colle difference à une subulivision en trois palties de propode 1 ).

Affinités des Pycnogonides. - Dans mon lavail sul los lyeno-

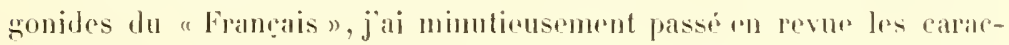

(1) Les épines modifices qui se trourent au borıl interne des uquatre derniers articles, sur les ovigères, sont les homologues des ipines siluées sur le tarse ot le propode des pattes daus

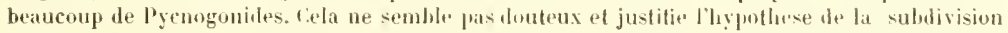
du proporle dans les origites. I'aprés Y. Loman $(1908,19)$, la premirte arlaptation des origires fut d'abord sensmielle el caractirisée parla présence d'ápines modifiese; flle devinl ensuite purement sexuelle. 
lieres qui permetrent de rattacher le groupe à la gramde classe des Arachnides, tolle que l'envisage M. Ray Lankester'

Ces eonsidérations 1906 ${ }^{\circ}$. 7-11, n’ont rien perdu de leur vateur, of je crojs utile de les relexer intégraloment ici, car, on le verra plus loin, ce probleme soulive encore des litiges.

A l'exemple de plusieurs zoologistes, ot notamment do M. Ray Lanhester (1), je considere les l'yenogonides comme appartenant il la grande classo des frachnides, qui se distingur essentiellement des Cirustacés par lar prosener, chez l'adulte, d'une seule prite dappentices próluceaux.

Ainsi comprise, la classe des Arachnides sétend drpuis les Trilobites jusyu'aux Acariens, en passint par les Xiphosmeres ef tous les Arachnides normaux. Elle jrésente ainsi un asseg grand polymorphisme, foufofois

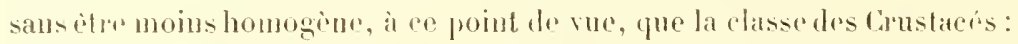
alsblaction laite des formes tris moditions par le parasitisme on par la fixation, il ne me parait pas a avoir plus de diflirener entre un fribolite

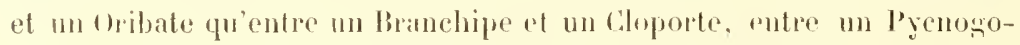
nisle et un Palpigrale fuentre une Caprenle ef un Ostracode.

"Les P'yongonides se distinguent par beaconj de caractères qui appartiennent également à cortains Arachuides les plus normaux : ils ont souvent, des elublicires triarticulée et en pince comme les Seorpions, les Palpigrades et les cpilionides; une tromperéduite à létal larvaire et, dans toms les cas, homologue de la saillie luccalr des léelipalpes, des Chernetres, des Galéodes ef surtout des Pafpigrades; des palpes phriarticulis of raisemblablement tatiles, romme un tres grand nombre d'Arachnides, et d'aillemes sins ancume refation avec l'alprareil buceal, conme coux des lalpigrades. Leurs appendices de la troisième paire sont

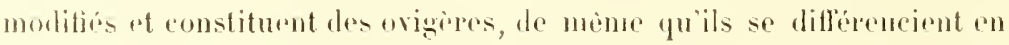
appendices palpiformes chez les l'ódipalpers.

"Les ovigires des tyenogonides serwent le plus sourent à porter les unfs, comme les appendices des paires antericures chez les Iformopula,

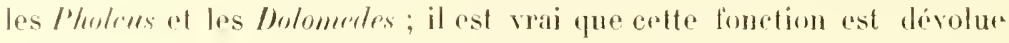

(1) L. Ris Laskistek, The structure and Classitication of the Arachnida (Ouart. Journ. mie. science, iol. XLIIII, part. II, p. 163-269, 190'). 
anx males des Pyenogonides, mais M. ILork (I) la vue remplio par les

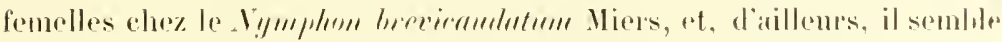

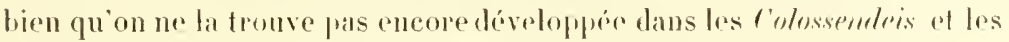
Merolepurle, ainsi ynol’a ubservé M. IIorgson 1905c).

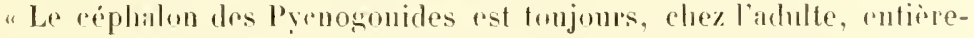

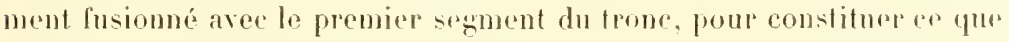

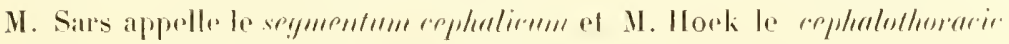

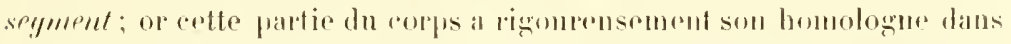

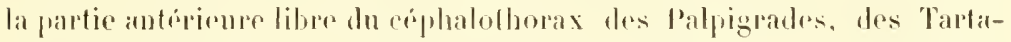
rides ref des solifuges; lien plus, cher les Solifuges, on tronve l'épuiva-

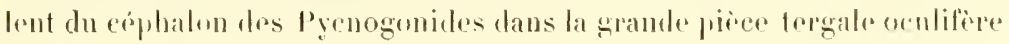
qui se paltache, par une ligne de suture, an tergite etroit des pattes de la

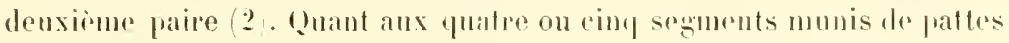
locomotrices qui constituent le trone des l'yenowonides, ils correspondent and trois segments thoracigues postérienrs des Arachnides normanx ef aux segnents qui lenr font suite sur l'aldomen, segments qui sont appendieulés rhez l'embryon et parfois mème choz l'adulte opercule gronital et peignes des seorpions). A cerpoint dr vur encore, il y a quelques ressemlibuces entre les l'yenogonides et crelains Arachnides; chez les Opilioniles notamment, wì le gramd tergite ciphalo-thoracipue

(1) P. P. C. IloEk, loc. cil., p. 135.

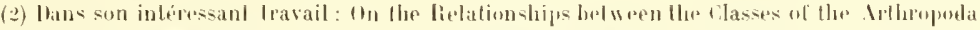

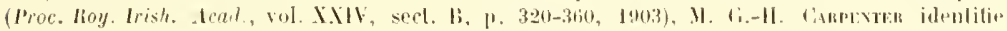

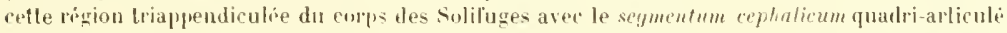
des Pyenogmiles; el, d'un coté, s'appuyant sur celle identitication, de l'aulle sur la présence

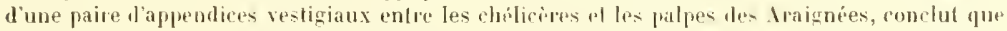

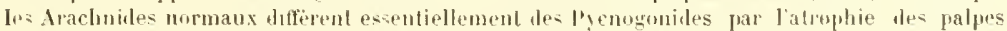

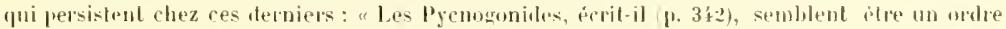
aberant d'Alachnides. Non seulement leur hicloire endegogénique, telle que l'a dérite .llor-

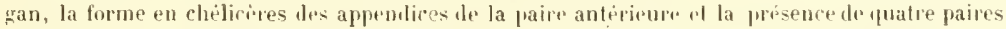
de paltes ambulaloires suggient des allinilis arachnidinnes, mais ausi le fait que lessegments portant les trois paires de patles pontérieures, flez les diserses familes de l'yengonides ronme chez les solifuges, ne se fusiommont pas avec le segment céplatique qui porle les quatres paise-

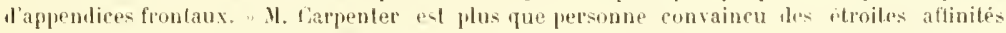

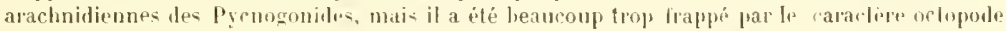

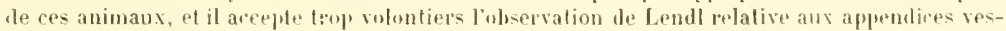
ligiaux des Araignies. Guanl a l'identitication qu'il propose, elle est sirement moins vraismhlable que celle oil liun voit dans le segmentum erphaliem des Pyougoniles l'homologue dt" la partie anterieure libre du ceplualothorax des solifuges, des Palpigrades et des Tarlarides. Au suplus, quand il ierivil son mémoirr, M. Carpenles ne eornaissait pas les l'yenogonides diraporles. 
est rontluent avec les tergites abdominaus et frarfois mème se confond avec les plus anteriours de ens deruiers.

"Les pattes des Pyenozonides me paraissent construites sur lo meme plan que enlles des Arachnides, mais d'un type phus primitif, en er sens qu'ancun de leurs articles ne devient épisternal l et pu'elles présentent de ce lait trois articles basilates bien distincts, les trois articles coxaux. thezles Arachnides, lestenx premiors artieles coxaux semblent fusionnés en un senl. qui est toujours plus on moins épistermal; pourtant it n'en us jas encore ainsi chuz les Limnles, on le premicr article roxal est eneor distinct, mais rémi au snivant par une suture immobile, le troisime article coxal jouant le rólo de trochanter. En fait il me semble fuion pent identilier comme il suit les divers articles des pallos chez les Arachnides normaux et les Prenogonides:

\begin{tabular}{|c|c|}
\hline Prowngentides. & Aractinides normaur. \\
\hline $\begin{array}{l}\text { ge } \\
\text { ge coxil } \ldots \ldots \ldots \ldots \ldots \ldots \ldots \ldots \ldots \ldots \ldots\end{array}$ & \}Hanche ou cuxa. \\
\hline Be $\operatorname{cox}: \ldots \ldots \ldots \ldots$ & Trochanter. \\
\hline Fenrur........... & Fémur. \\
\hline Prentipr tibiat. . . . . . . . . . . . . . . . & Patelia. \\
\hline 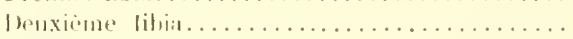 & Tibia. \\
\hline 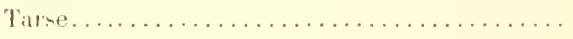 & Nletatarse. \\
\hline Propmile.................... & Tarse. \\
\hline
\end{tabular}

"Cir yni domne me récle valeur is ontre interprétation, c'est lo fait que les opilices des grlandes sexuelles des l'yenogonides s'olservent tonjours sur le deuxirme article coxal of les orifices des wlandes coxales des Arachnides norman sur la hanche. En lemr qualité d'organes segmentaires néphridiens, les glandes coxales des Arachnides sont trís propres à s'adapter aux fonctions vectrices génitales, et ce qui porto à croire qu'olles remplissent bien réllement ce röle chez les Pyenogonides, cest l'étrange ressemblance que présentent les glandes génitales de ces Arthropodes avec les crlandes coxales des limmles. Wur l'on compare à ce point de

(1) J'entends par article rjisternal un attich tui se fusionne largement avec le corjs et joue

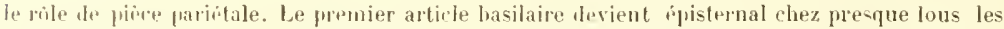
Afluopules un peu flevios en uranisation; on lobserve encore plus on moins apparent dans les patlu qui ont conservé nu caractipe prinitif (Blattes, Aroules, frattes abdominales des crus. facris), mais te plus souvent if perd toule indépundance. - Voir à ce sujet : pour les Blattes, I. Wour- Vasm, Morphologiral Voles braring on the origin of lnsects (Truns. ent. Soc. London, 1879, p. 1:i6i), et pour les Crustaris, H.-J. Hanex, Zur Morphologie der Ciliedmassen und Mnnd-

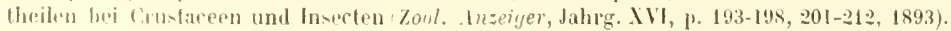


vur les glandes coxales de Limulus polyphomms, telles que les a tigurées Packard I, avec la description et les figures des glandes génitales dommés par M. Hock 2) pour les Pyenogonides. Mstraction faite de l'anastomose postérinure, qui rémit les glandes cosales des deux còtés, cest rexactement la meme disposition anatomique. Il est viatque lespores sexurls des leprogonides se trourent soureul mais nou toujours sur plusieurs paires de pattes, et parlois mème sur toutes, tandis que les oritices coxaux des Arachuides se localisent sur les appendices de la troisime on de la cinguieme patre; mais on peut prenser pue ces orifices étaient plus nombreux ehez les Xiphosures primitils, et d'ailleurs on sait,

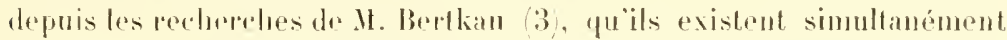
sur lestroisiome at cinquiome appendices dims les Araigmás thriaphoses du gemre Jtypms.

"Comme la plupart des Arachnides. les lpyenogonides présentent sur l’intestin moyen de nombrenx prolongemenls cureaux, yni pénitrent dans les pattes comme ceux des Opilionides ; cette pénétration est vraisemblablement la consépuence de la réduction du corjus dans le sens transversal, mais un me saurail en dire autant de la prósence mème des carca, ot le fait que ces dernirs existent chez les l'yenogonnides comme chez prestue tous les Arachniles normans semble lien indiquer, chez ces Arthropodes, une origine commune. J'ijoute que les yeux des Pyomognides sont du meme type que les geus midians des Arachnides normatux, ef yue les spermatozö̈des ont la forme ordinaire liamenteuse dans l'un ou l'untre groupe. On sait que ee dernier cal'actire n'existe pas dans les Crustacés, sauf tontefois dans lorde des Cimbipiodes.

"Faut-ilajouter pueles Pycuozonides ont desmitamorphoses romme les Acariens of que leurs laress ou formes mulyenuares libres présentent, comme, chezertains de aes derniers, des phemomenes d'atrophie at de regrinération de membres? Che\% les Gamasides ef less Ixodes, ae sont los

(1) Voy. à ee sujet la ligure schématique lires du mémoire le Pachard par M. Lay Lankester dans son Iravail sur Ia slruclum ef la clascification des Arachnides f(umet. Journ. Micr. Science, 1ol. XLIIII, jail. I], fig. 28).

(2) P. P. C. Huk, loc cit.. J. 12x-132, et P]. XXI, Jigr 10.

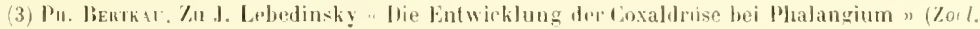
Anzeigr, Bd. 11, 1\%, 1\%, 189!). 
pattes de la quatriomm paire qui dispatraissent dans la forme ambryon-

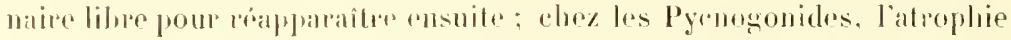
pertesur les appendicesde la deux iemeed de la troisiome paire, qui réappa-

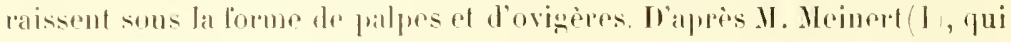

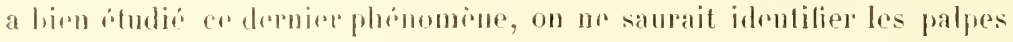

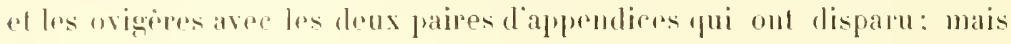

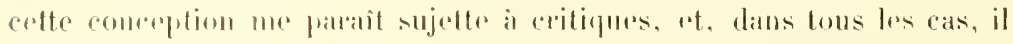
convient d'attribure aux mèmes somitrs les"membres do l'alulte el ceux las la furme embryomairo 2 .

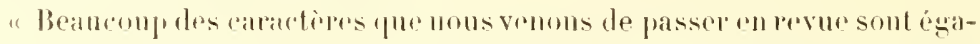

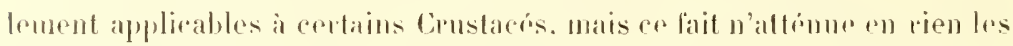

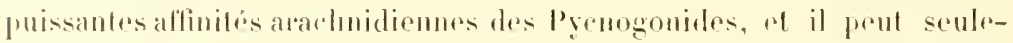
ment servir ì pouver yue les Coustaces at les Arachudes somt issus d'une souche commone 3 , les premsers aver denx paires dappendices prébureanx, les seconds avere mo srule paime.

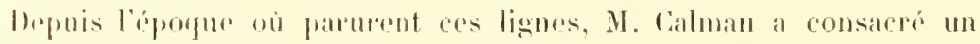

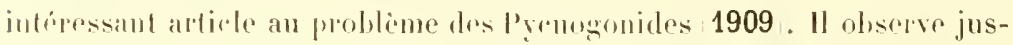
tement que ees animans we presentent ancune alfinite directe aree les

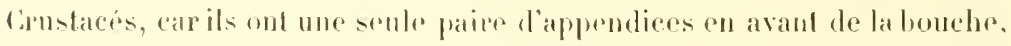

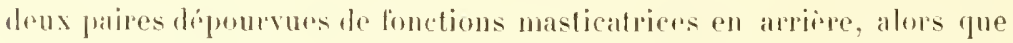
les Cruslaces ont deux paires f'appendices préluceaux ret au moins frois paires d'appendiers post-loncraux, dont los artioles hasilaires jenent un

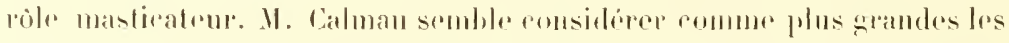
allinités des l'yenogonides ef des Arachnides, mais me va pas foutrofois

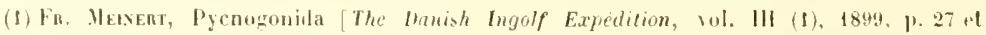
suis.

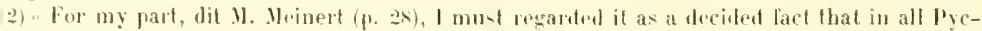

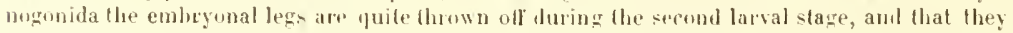
are in no way identical with the latier imagnal line limbs, the palps and the origerons legs. "hich latter also, and of this there is no douht, arise, although on the same metamer. atill in wher pars of thrse metamerm. "

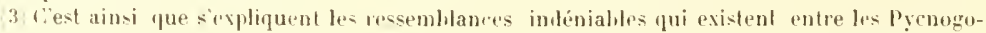
nides et les Cruslacès, surtoul a l'état tarvaire. Cis resemblances ont élé fort bien mires en

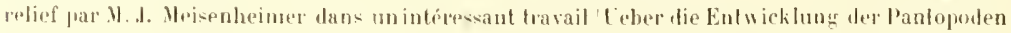

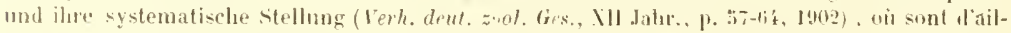

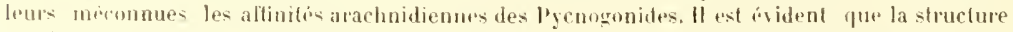
mp pince des chélicires est d'urigine sucondaire par raport à la souche commune des drachno-

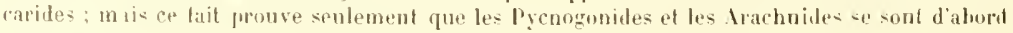
adiptes dans un sens of les Crustaces tans un autre. 


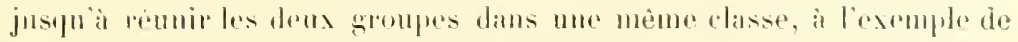
Mll. Carpenter of liay Lankester. Wi troures, en efled, chez les Arachmides, les dens pares d'appendices post-luecaus palpes et ovigimes qui

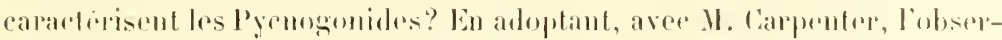

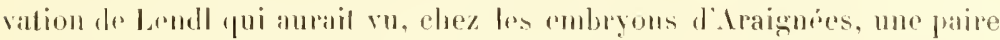

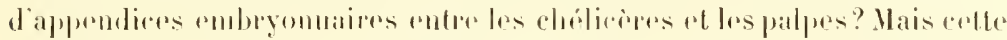

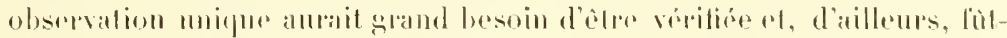

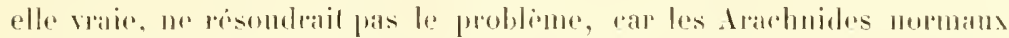

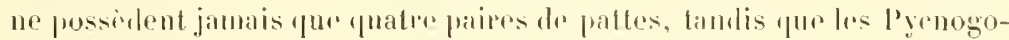

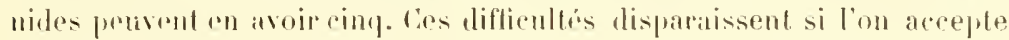

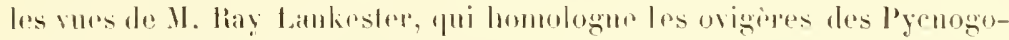

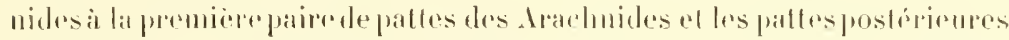
des premiers and appendices antépioms de l'aldomen des seconds. Maris

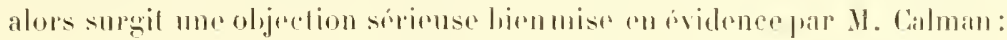
"Lat distinelion ontre les segments prosmatiqurs ot mésosonatiques,

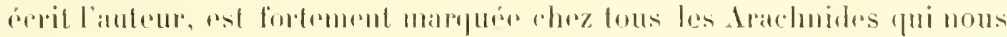

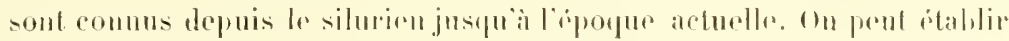

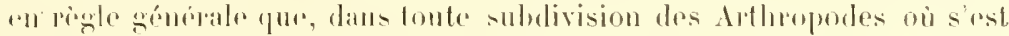

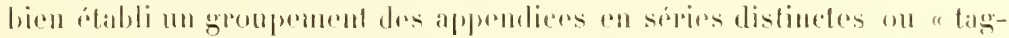
mata ", re groupement soblitipe rarement tout a fait, sinon jamais, an rours del'évolution ultérienre da groupre... lles lors, lidentité alsolue de

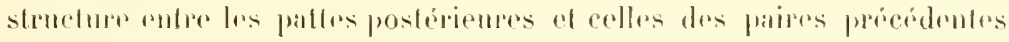
chry les Pyonogonides semblerait impliquer dans les vues do M. Ray

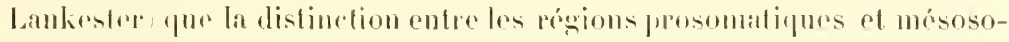

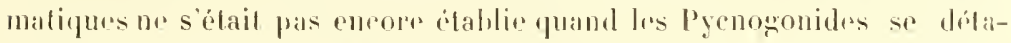
chiopent du trone principal des Arachnides."

On me saturait mirux dire. M. Lalman observe loutefois que celte con-

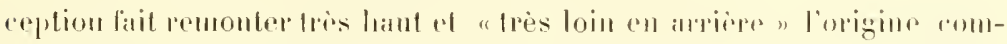

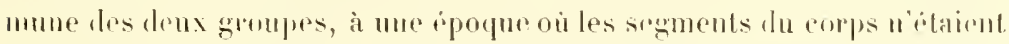

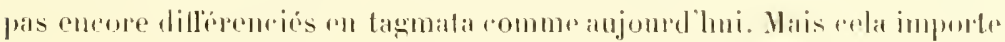
pen, si l'on adured avee nous qu'il convirnt de panger dans le phylum tes Arachnides lous les Arthropodes qui furem on qui sont actuellement munis diune paire diapendiees prélnteranx aver des appendiees post. buceanx dont lesfonctions maxillaires sont réduilesou mulles. Ainsi caracté- 
risi, re phỵum apparait hien distinct de cetui des Crustacés, dont il se mapproche certainement frar l'intermediaire des Trilobies; il est flus distinct encore de enhi des Trachéates, encore que les denx groupes de cephylum. les Insectes of les Ityriapudes, airnt anssi divergi de bome heure, c'est-i-dire arant la spécialisation segmentaire en thorax et ahdomen.

Nous roici dente conduits à ranger les Pyomgonides dans lir classe des Arachnides, folle que l'envisage M. Ray Lankester. Cie savant $(1904,213)$ a divisi la elasse end drux siries: les lummmméristipnes, dans lesquels to nombre des somites est rariable. "l les Vommorirtiques, ou ce nombre est primitivenent constant. A yant tantot cimp, tantot quatre somments prolifires, les l’ycnogonides dewaiont se ranger dans la premiere sépir, comme les Triblites, mais ils se distinguent profondement de ces derniers ef se rapprobluent davantage des Arachnides normaux à rause do leups chélicieres, de sortr que tres denx sépies proposés par M. Ray Lankester semblent peru naturelles. On peut en conserver les termes salus leur dommer une valenr systimatique, ansi que j’avais re devoir le fare dans mon étude sur les Pyenogenides du "Français ".

Comme je lécrivais dans ce dernier travail, je ne crois pas gu'on puisse voir dans les lyenogonides des formes dégénérées; "ce sont foul simplement des drachnides primitils ayant subi une adaphation spéciale n. dai montre plus haut qu'ils se rappochent des lomes ancentrates du

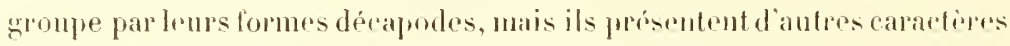
primitifs parmi lespuels on doit citer" "ha persistance drelarticle basilaime des pattes, l'independance de eet articte par rapport anx suivants of aux parois du corps, la répétilion métancipigue des prolongements sexuels, des pores coxamx et des circa digestifs, enfin ot surtout la structure scalariformede la chaine nervense ventrale. Cedernier caraction, à luisul. sultirait pour atablir que les Pyenogonites sont des formes primitives ; il dilfire tolatement du systime nervenx condense quon olsope chry

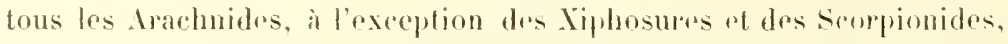
qui sont, eux aussi, tries rapprochŕs de la souche commune " $1906^{\mathrm{b}}, 12$. 


\section{CILASSIFIUATIUN}

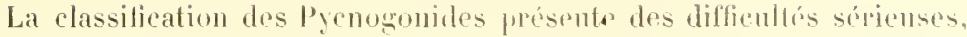
qui sont dues, pour une part, à l'homogéneité apparente du grompe, pour une autre à ka valeur systruatique douleuse des caracteres qui diflorencionl

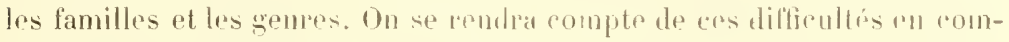

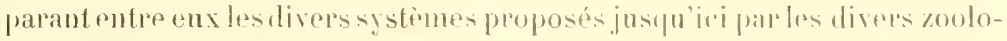
gistes pour ílablir des sublivisions dans le groupe.

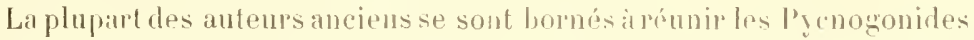
enfamilles, sans s'accorder au demenrant sur les gemers qui drvaient entror dans ces dernières. A umerpoque plus récente, ou a tenté des groupemonts basés sur les caractères des ehélicizes et des palpes. L iun de cos systèmes est celui propusé pra. Wilson, qui divise les l’yanogonides anmme il suit 1880, 469 :

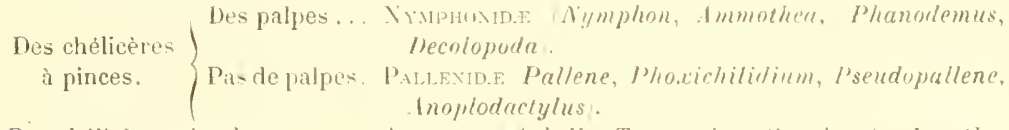
Des chélicères simples....... ICHelid.e Achelia, Tanysty/um, lorniger = Lecytho rhynchus', Eurycyde. Ascorhynchus, Paribaa).

\section{Pas Despalpes... Pasithoide Pasithoe, Emleis, Rhopalorhynthus of de chélicères. ' Pas de palpes. Prowononde E (Phoxichilus, Pycnogonum).}

Bien plus récemment, M. G. O. Sars 1891 a présenté mu systime and-

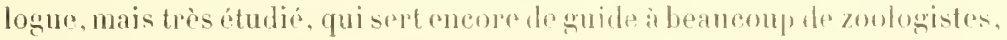

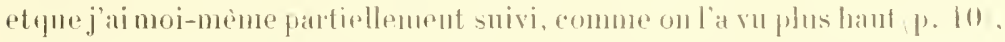
daus mes études antériemres. Inus a systime, les Pyenognuides sont divisés en troisordres qui comprennent les famillos of les gempes suivants: Ler ordre : ACIIELATA .... ( PrCxolionid E (Pychogonmm).

Pas de chélicires. Phoxichicto (l'horichilus).

PhosichlldDID. / lhorirhilidimm, Anoplodactylus

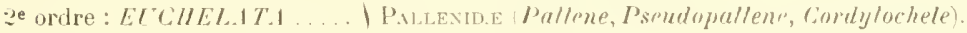

Chéliceres bien déveloglux's. I Mruphoxus: (Nymphon, Boreonymphon, Chatonymphon).

3 ordre: CRIPTOCIIEL.IT.1.

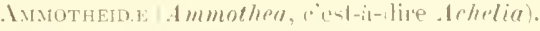

(Chẻlicères rüunites.

Ei ricitide (Euryeyde, Asrorhynrhus).

Pasithoid.e Golossenteis.

11. Loman a justement crilipué ces systimers, qui s'appuient sur les

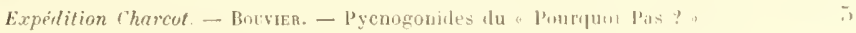


modiliealions dappendices rminnmment propres aus adaplations secun-

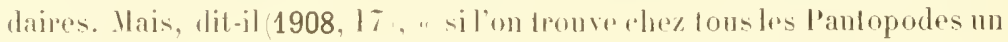
ongane particulier qui prosentedes modifications moliress sms, non pas

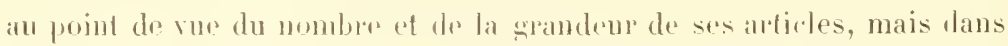

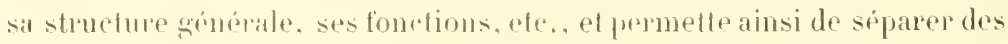

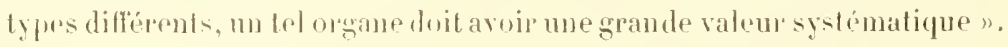
Or m senl organc lui parail arturlloment répondre à ces exigences ; il šagit des ovigiores du mile, qui sont ridomment des pattes adaptées à un

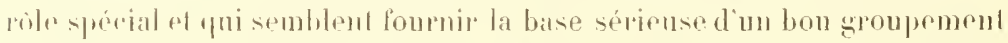

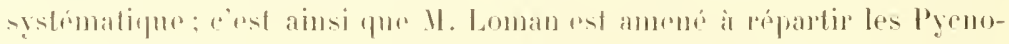

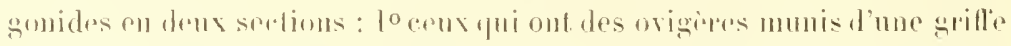

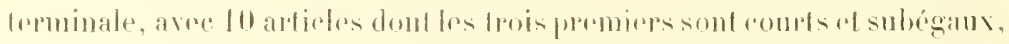

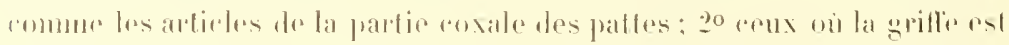

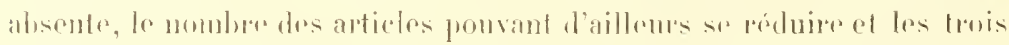

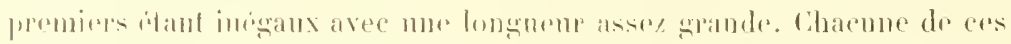

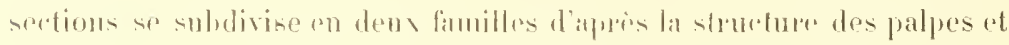

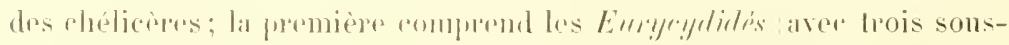

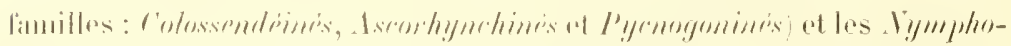

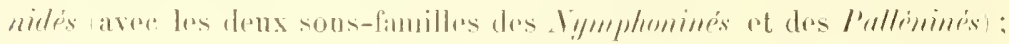

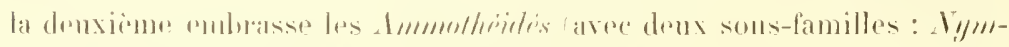

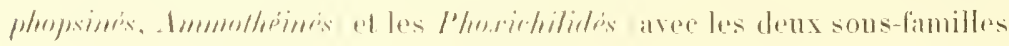

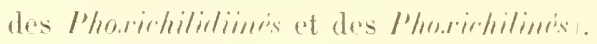

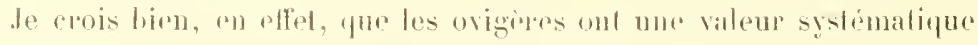

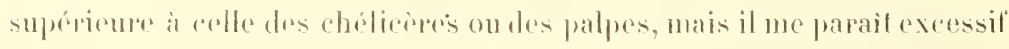
de leur subordomner toutr la classifeation des l'yenogonides, d'antant gưils prosuntent des passages, d'une section à l'autre, et que lrup utili-

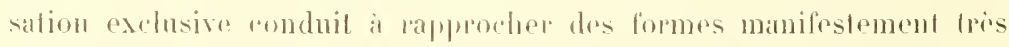

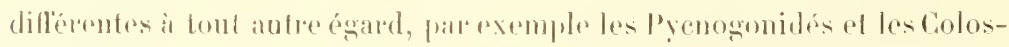

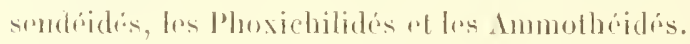

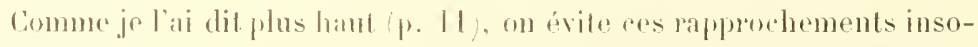

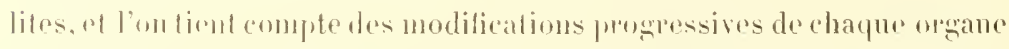
en divisant les J'yenogonides en series evolutives ayanl hacme pour point

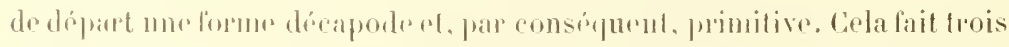

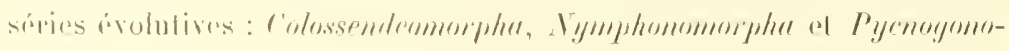


morphu; mais je crois bien qu’il fant en stablir mo puatrième, collo des

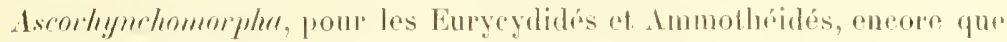
la forme déeapodo soit aeturllement ineonnue dans ce grompe. La sireie

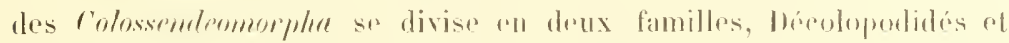

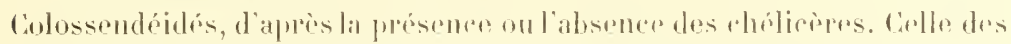

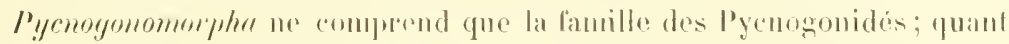
aux deux autres scolies, elles se sublivisent on familles d'apres les riegles

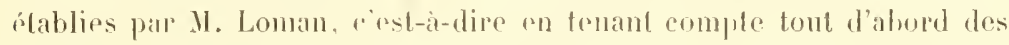
ovigères, el, apres coup, des chélicires of des palpes, comme on lo verra plus loin, dans la partio systimalipue de reet ouvage.

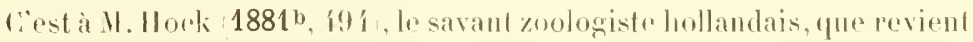

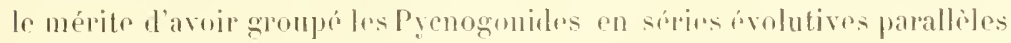

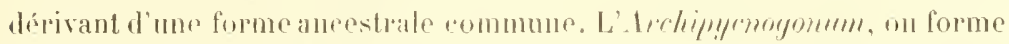
ancestrale imagine par M. Howk, mo parait très voisin du gempe Decolopodre, ainsi quo je l'ai montró dans me olude antérirme, mais il était supposé octopode contur tous les Pyomogonides commus à ecthe cépoque. Je ne sais purlle est loppinion de M. Ilork sur les Pyenogonides déca-

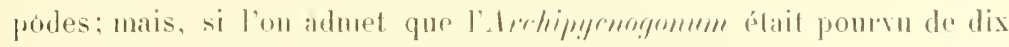
patles, le systime du savant hollandais jursente dans ses traits essentiels les momes raractieres yue le notre, car il divise les l’yenogonides en

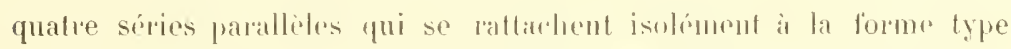

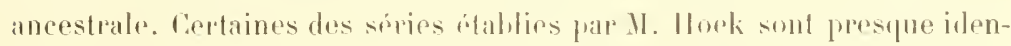

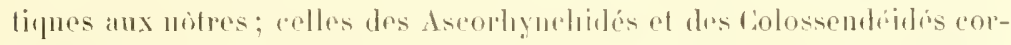

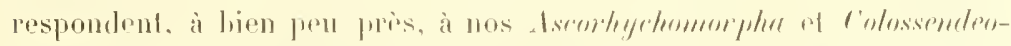

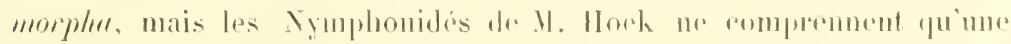

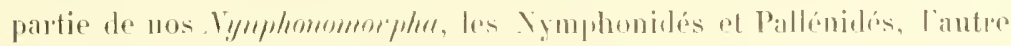

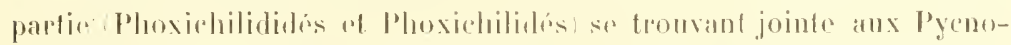
gonides, pour constitnepla tuatrieme sereie, cello des Phoxichilides, ynj

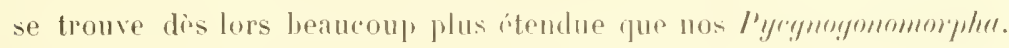
M. Loman a suivi l'expmplo de M. Ilow on séfratanl les Phoxirhilidiidés ef les Phoxichilidés du grompe des Vymplonides of des Pallenides, mais il a justement observé yue les l’yenogonidas ne présentent que des rapports de convergenee avee les Plowichilidris.

Il ne faut pas oublior que la classilicalion de 11 . Ilock remonte a I88I, 


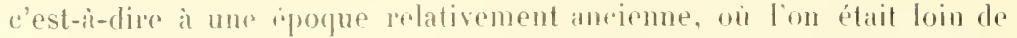
sompromner l'existence des l'ycnogonides décapodes: elle est par conséquent très en avanee sur son éporue, et on tloit la eonsidérer comme un essai dres plus mritoines.

J'ai montré plus laut p. () comment, après les helles découvertes

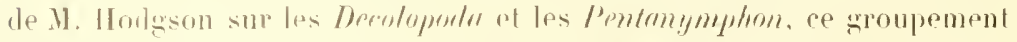
pll séries curolutives fut repris et modifié par M. Cole 1905 ot par noimême 1906 sons des formes dillórentes, l'une et l'autre sujelles à critique, mais intéressantesà cause de leur point de dipart. C'est à juste titre yno M. Calman a qualilié ces swoupenents, du moins colui dunt je suis l'autrur, de fomorl mnd mmmmral, mais les déconvertes du "Pourquoi Pas?" netairnt point concore réalisises. J'espère qu'on sera plus indulgent pour la clasilication suriale proposie à la suite de ces découvertes et dont je rrois devoir domer le driveloppenent.

\section{CLASSE - ARACHNTDA.}

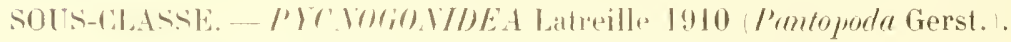
Podosomata Leach 1815: Pantopoda fierstäcker 1802.)

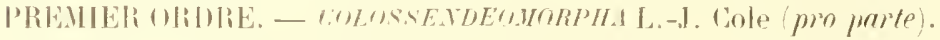

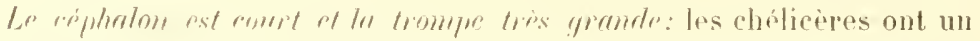
scape de deux articles, mais le plus souvent font défaut "liez l'adulte. Les

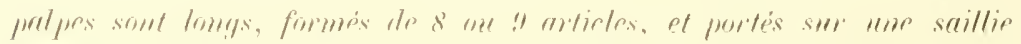

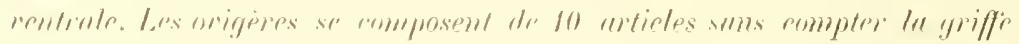

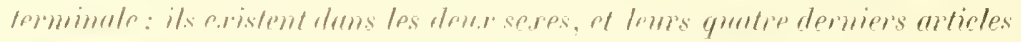

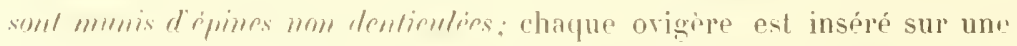
saillie vontrale analogue à rolle des palpes: les broses des dend origiores

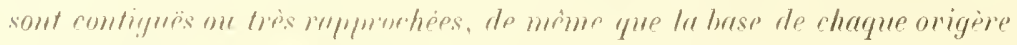

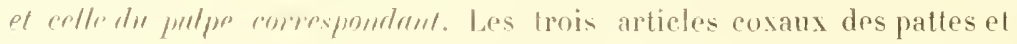
des ovigipes sont jursque tonjours forts, pen allongés, ot pris ensemble, beaucoup plus courts que le frimur. Il y a, dmus les dems seaes, m orifier

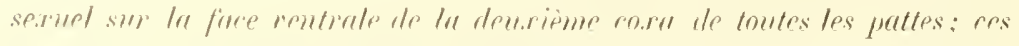

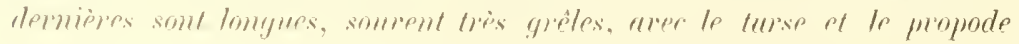




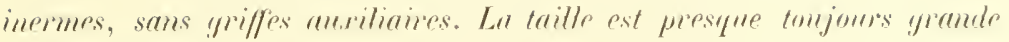
ou fort grande.

Le groupe se distingue an premier abord far la position relative des ovigères et des palpes, cesappendices tant tres rapprochés ou contigus à leur base, qui est formée par une saillie ventrale étranglée à la base. La saillie basilaire des palpes est considerée commen un article par la plupart des auteurs, M. Loman exeppté, et jai moi-meme précélemment suivi la règle courante ; mais c'est une simple protubérauee étranglée i son origine, non un article molile. Les Rhopalorhym hms semblent so distimgner par la position de leurs palpes, qui sont un jen bloignes des ovigipes; del est du moins ce qu'on observe dans mo figure de $V$. Loman représentant dr coté le Rhop. Krïypri Wood-Mason 1908, fig. 2lö; parcontre, les deux appendices sont représentés contigus à leur base dans unr ligure consacrée par M. Carpenter an Kh. rlurigpr Larp (1893, H. II, lig. 3); je n" puis trancher celte question, u'a yant pas ou sous los yeux des exemplaires le ce genre.

L'ordre des Colossendéomorphescomprend les deux familles suivantes:

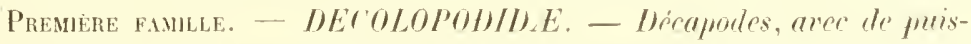
santes chélicères en pinces, dont lo scape commend deur articles. Palpes do 9 ou sarticles. Un seul genre: Decolopoda Eights 183 't.

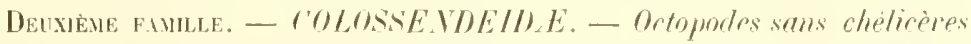
et ì palpes de 9 an 8 articles. La famille se divise en trois genres: rolossendeis Jarzynsky 1870. Rhopalomymehms Wood-Mason Is73 et Pipetto Loman 1904; elle se relie aux bécolopodidés par les folossendeis, qui ressemblent tout à lait aux Decoloporlı, saul les chólicères el le nombre des pattes.

\section{DEUXIEUE ORURE. - NY.MOHO.HORPH.I R. I. Pocok emend.).}

Le céphaton est ordinairement allonge of le cou hien distinct: In trompe

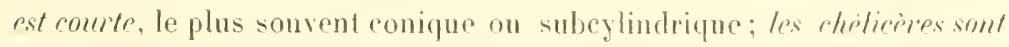

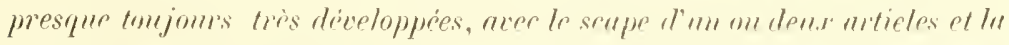
pince ramenée rn auant de la bouche. Les pulpes ne somt lien developpess que alans les formes primitives, oin ils comptent is articles, rurement i; 


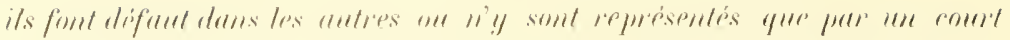

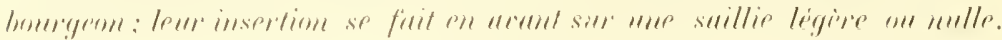

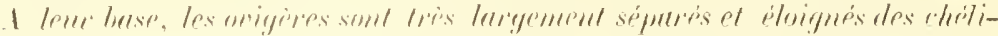
cores : clow les formes primilives, ils se composent de lo anticles sams compler la sriffr tominale. of les qualre apticles qui précident cette

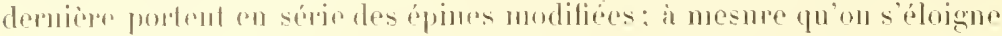
de cos formes, on voit la gepiffe temminale al les epines modifices dispa-

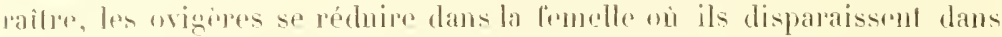
certains cas completemont. Les trois allicles enxamx des palles sont de longurne variable, mais d'osdinaire plus longs que dans lo groupe pré-

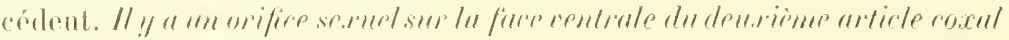

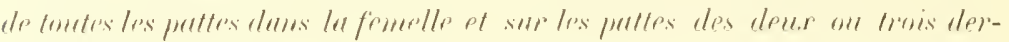
mières paires dems le mile. Les pattes sont de longuemr variable, avee l.

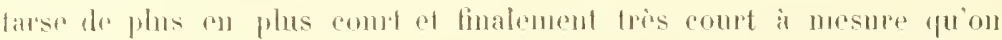

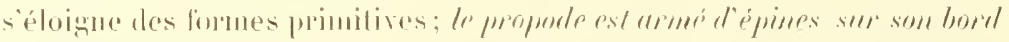

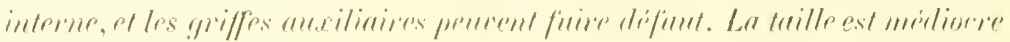
ine petits.

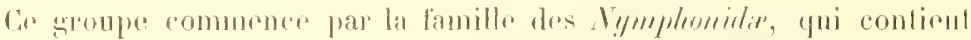
nueore une formo primitive décapodr, le zempe I'entumymphom, et dont

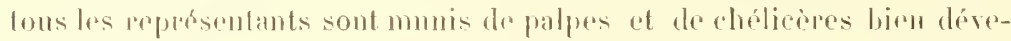

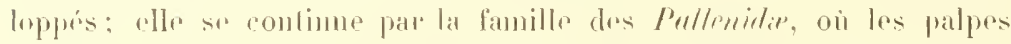
manchent prespne toujours el mstent rndimentaires quand ils rxistent. La

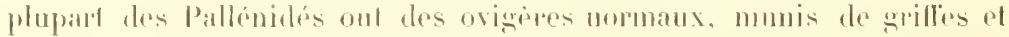

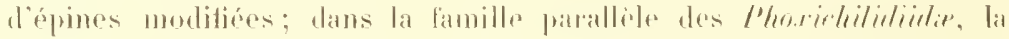

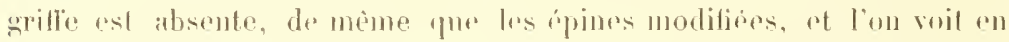

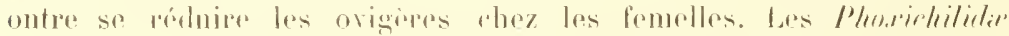
ocenpent le sommel du grompe i la snite des Phoxirhilidides, dont ils se distinguent par la disparition totale des chéliceres dans les denx sexes, parl'alsence des ovigieres thez la limolle el par la réduction de ces appendices a sept anticles chez le mâle. Les Phoxichilidiidés ne dérivent probablemenl pas des Yymphonidés anduchs, mais de guelque forme phos primitive, far los chelieires do cortains d'entre enx ont un seape de deux arlicles.

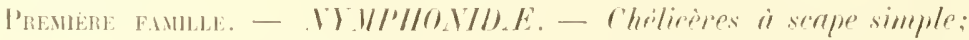




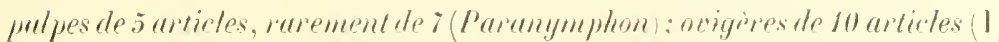

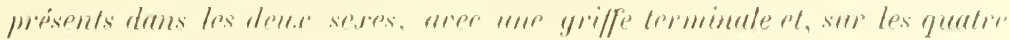

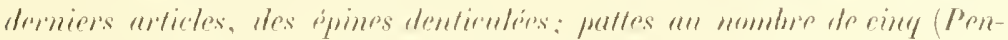

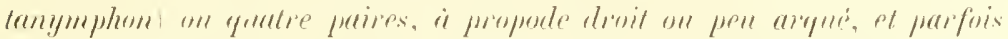

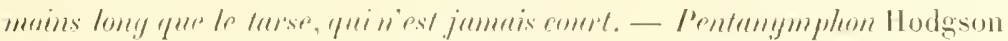

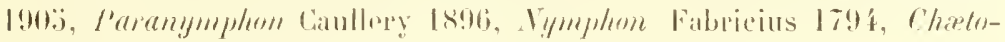
mymphm" G. (1. Sars I888, Lim'rmmymphom G. O. Sars 1888.

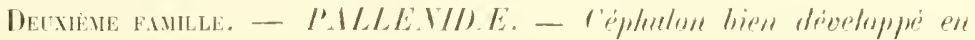

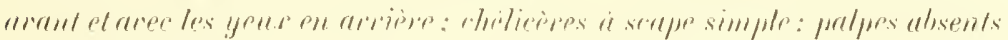

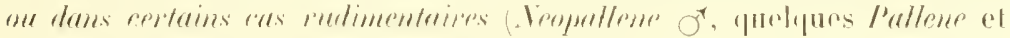

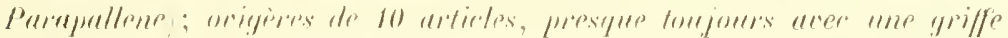

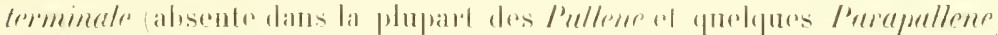

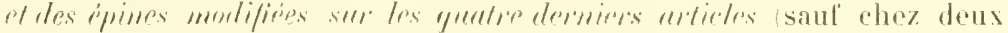

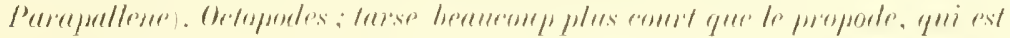

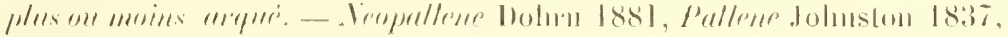

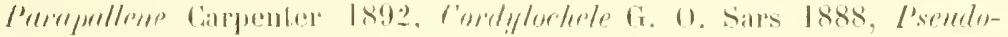
pullemen Milson เระล.

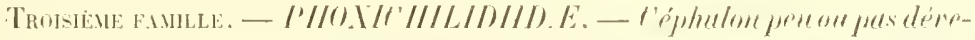

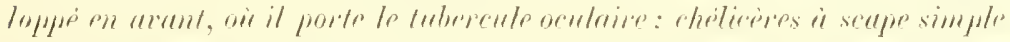

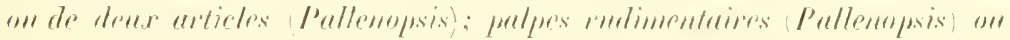

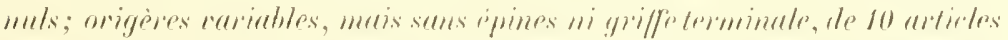

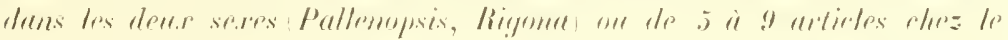

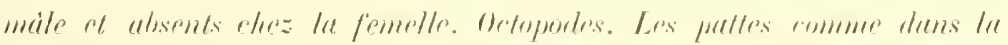

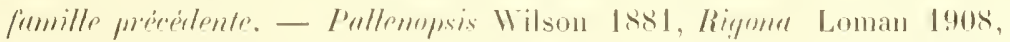

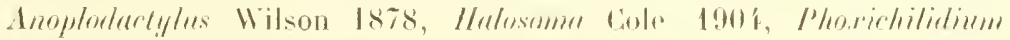
II. Milno-Edwards IS ito.

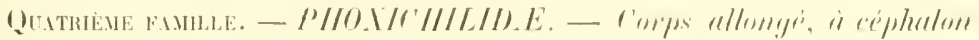

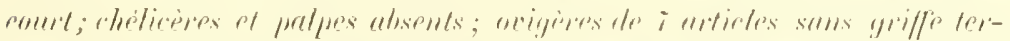

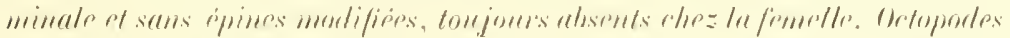

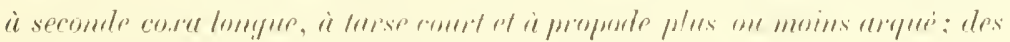

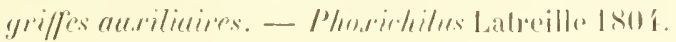

(1) Caest à Lort quion attribuait jusqu'ici aux l'artunymphon dus ovigères de 8 ardicles; M. Loman vient de montrer 1912 , i) qut ces articles sont au nombre de 10 , sans comples la griffe terminale, 
Cet ordre renferme lous les Euchélates de II. Sars, avec, en outre, les Phoxichilidís que le mème auteur rangeail dans ses Achélates à côtí des Pyenogonidés. M. Hoek 1881 b ot M. Loman 1908 le divisent en deux groupes, qưils séparent profondément l'un de l'antre; le premier groupe pomprend les Tymphonidés ef les Pallénidés. qui forment pour 11. Hoek une sere spéciale tet que 11. Loman rapproche des Euryeglidés: freseond seitend aux deux autres familles que M. Hoek réunit dans une meme série avec les Pycnogonidés at que .H. Loman rapproche des Ammothéidés. Pour justilier cette division en deux partios, M. Hoek s'appuiesurla struteture deschéliceres, dont le scape se compose de deux articles cher les P'ullempsis, el II. Loman sur la structure des origères, qui sont dépourvus de grille terminale dans les Phoxichilidiidés et lis Phoxichilidés.

On ne saurait nier l'importance do ces denx caracteres, et jai atopté les rues des deux excellents auteurs, mais sans aller anssi loin qu'eus: les fualre lamilles prósentent un facies commun ef me paraissent dériver d'une forme primilivequi avait leschélicires des Pallenopsis et les palpes des Nymphonidés; les Nymphonidés et les Pallénidés se rattachent à cette forme dont ils ont conservé la griffe ovigérienne, mais non le scape à deux articles; l'inverse s'est produit dans les Plıoxichilididés et les Ploxichilidés qui ont perdu la grifle des ovigères, en conservant parfois Pallemopis: le scape chélicérien des denx articles.

ThOISIEUE ORIJRE. - ASCURHYTCHOMORPHA R. I. Pocock (ement.

Le céphaton est tries variable. La trompor est gramle et forte, somerent

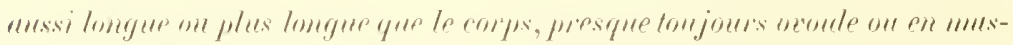
sue, ol fréquemment itmenép ohliquement en arrière an-dessous de la fure mentrale; less rhélicripes som rétuites, rulimentuires on mulles: elles dépassent rarement lo buut de la trompre. of ulors lemes pimess ue sont pas ramenées en avent de lu bourler. Les palpes varieut beancoup, tantò plus longs que la Imomper formes de lo articles, puis deplus all plus courts, avec de moins an moins d'articles: ils ne font défaul que dans un seul genere

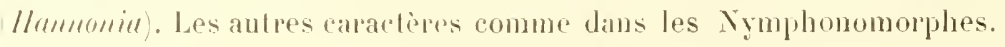




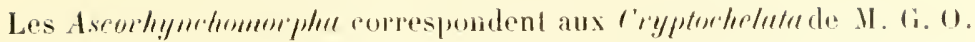

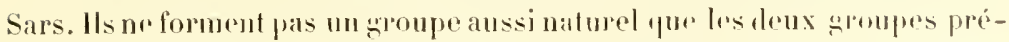

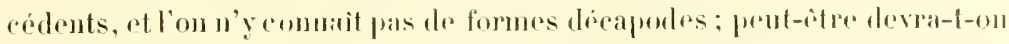

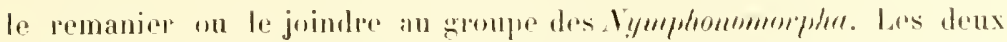
lanilles réunies daus les fscorhynrhomorphor ne semblolent pas absolument indépendante loune do lantre, et les Nymplopsimés paraissent

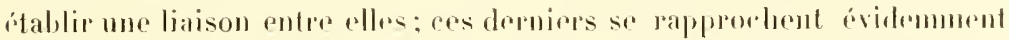
des Ammothéiues, ot M. Luman lo plare justemenl dams la mome lamillo:

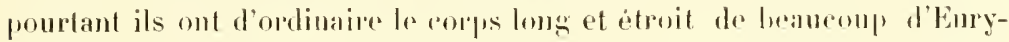

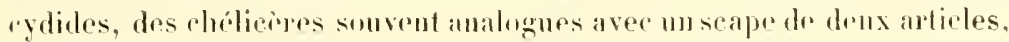

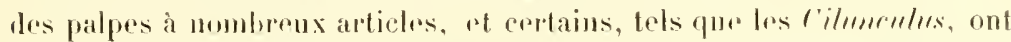

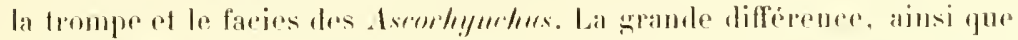
l'observe ll. Loman, esl due a la slouturedes ovigeres, qui ont une grillo

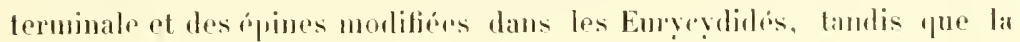

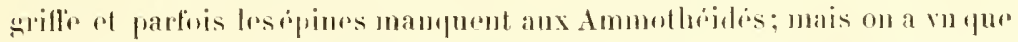
ces caractères se modilirul marluellement clue\% lis Nymplumomorphes.

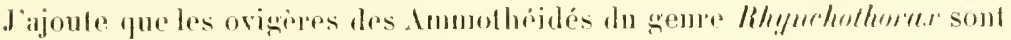

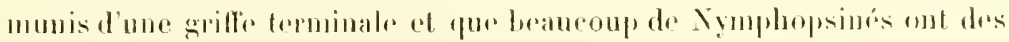
épines ovigurienues dillïrencices.

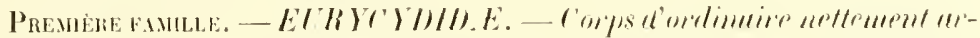

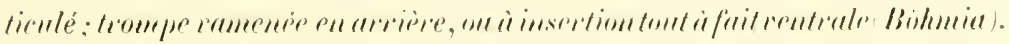

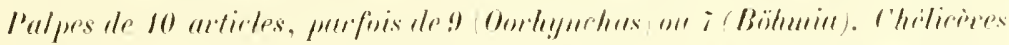

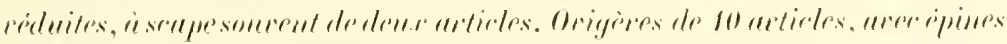

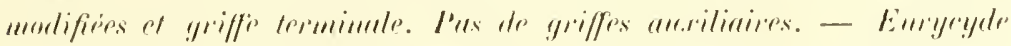

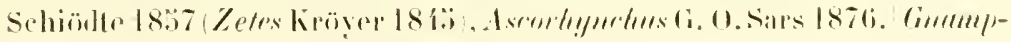

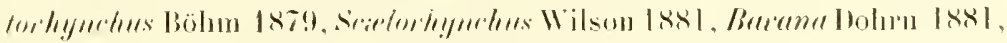

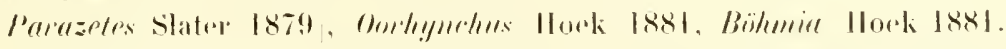

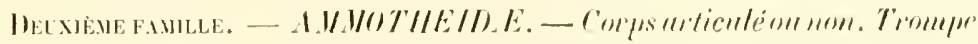

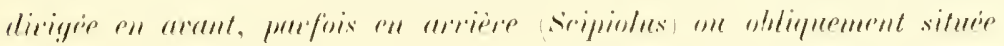

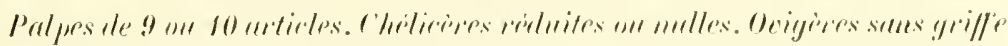

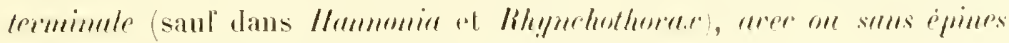
molifiees. Prestgue tomjumes des griffes andriliaimes.

Avec M. Loman, il comvient de diviser les Ammothridesen deux sousfamilles. 


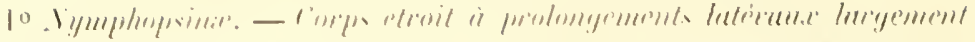

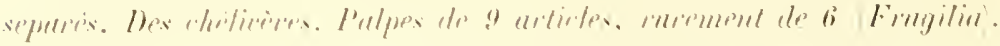

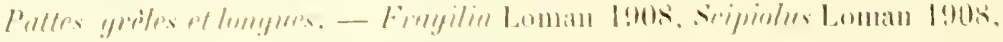

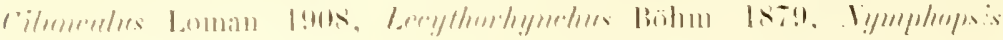

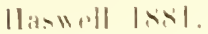

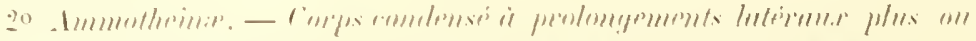

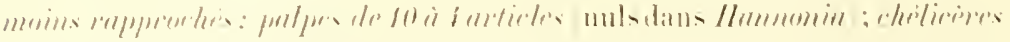

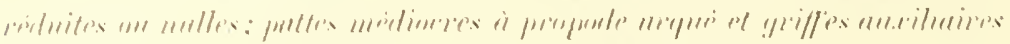

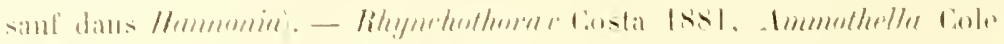

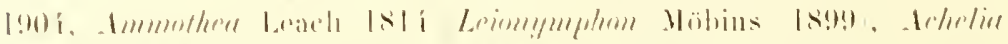

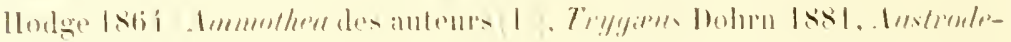

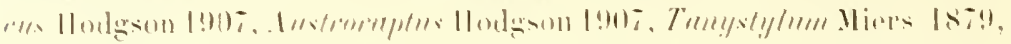

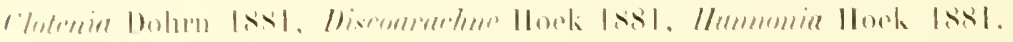

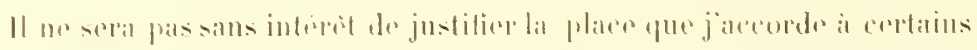

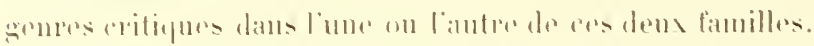

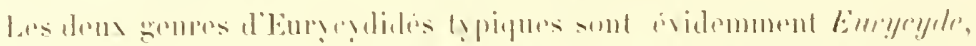

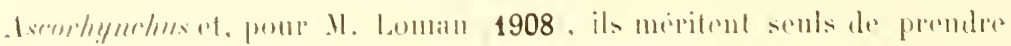

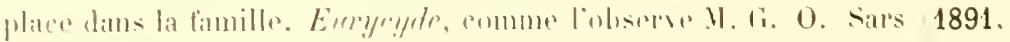

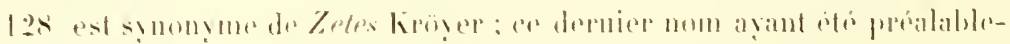

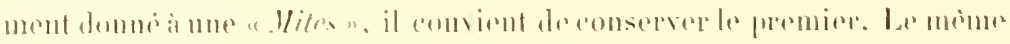

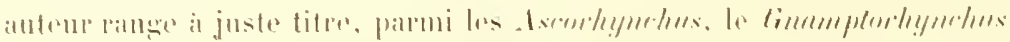

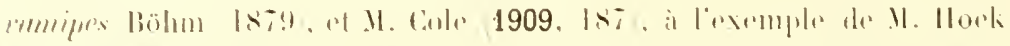

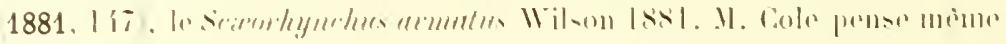

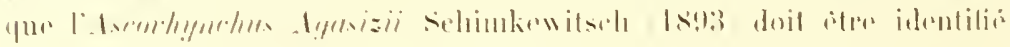

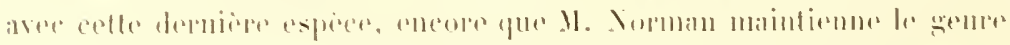

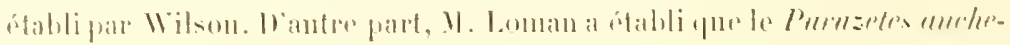

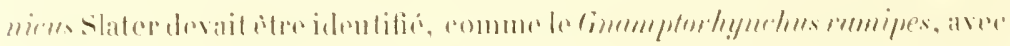

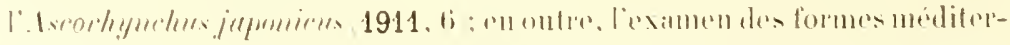

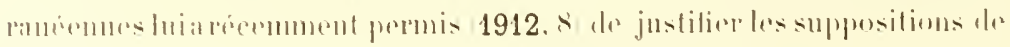

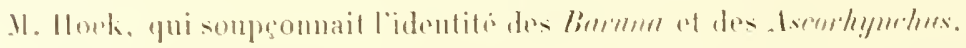

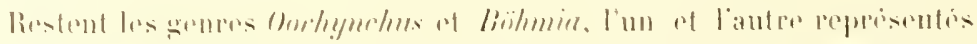

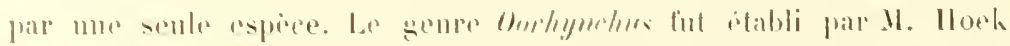

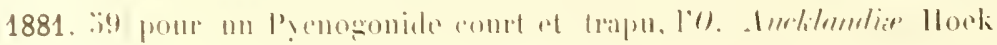
(1) Voir in. 


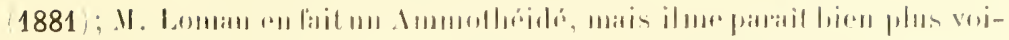

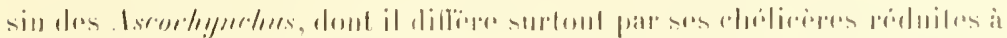

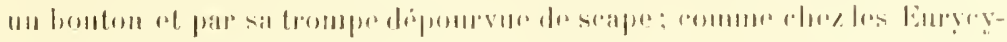

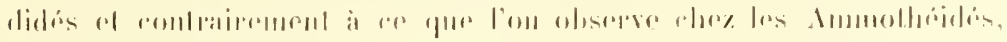

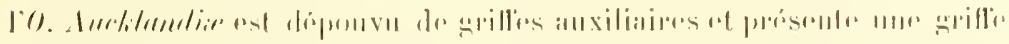

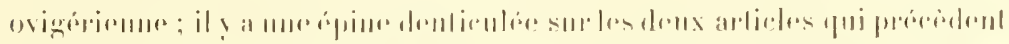

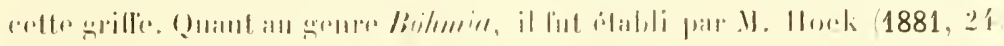

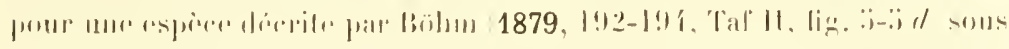

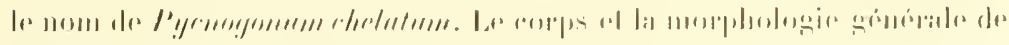

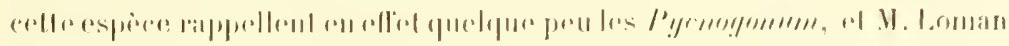

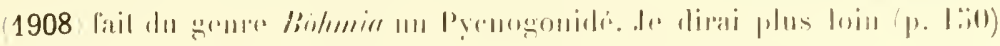

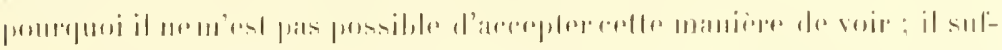

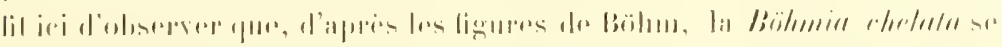

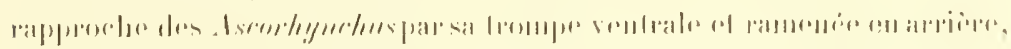

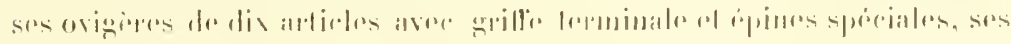

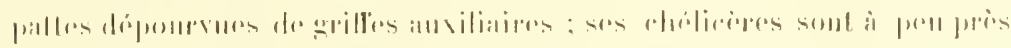

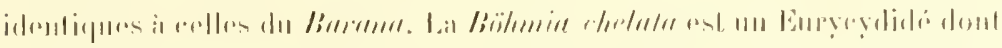

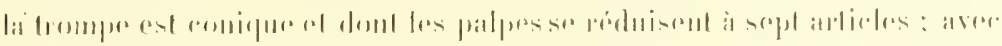

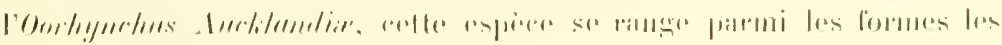

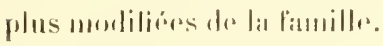

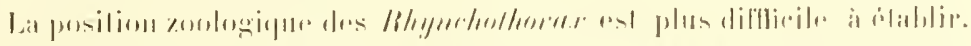

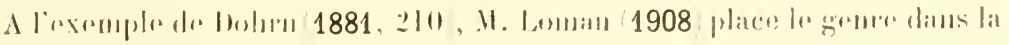

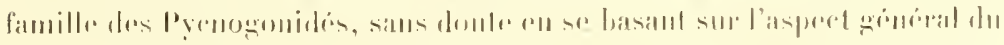

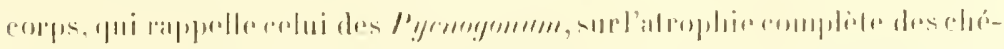

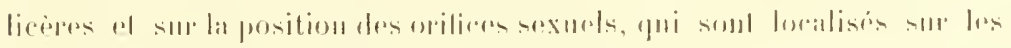

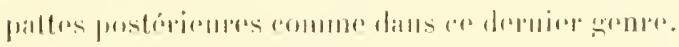

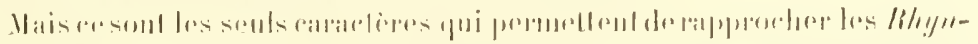

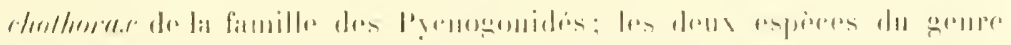

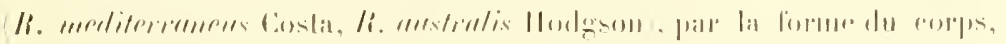

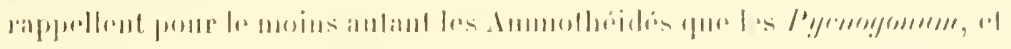

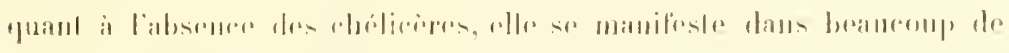

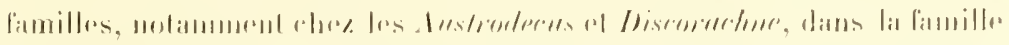

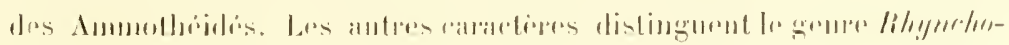

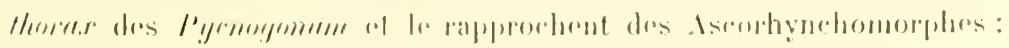




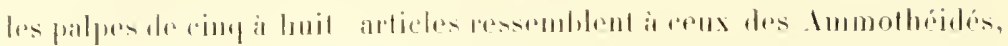

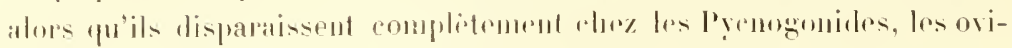

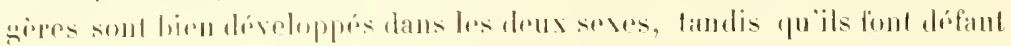

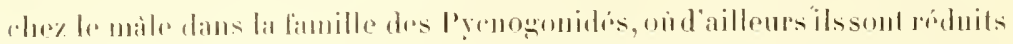

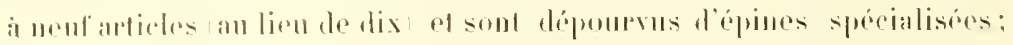

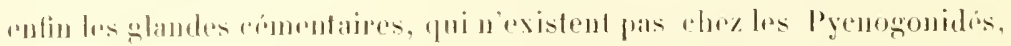

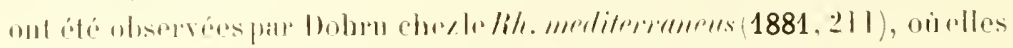

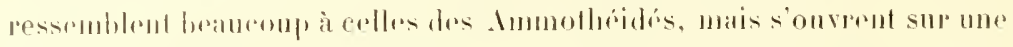

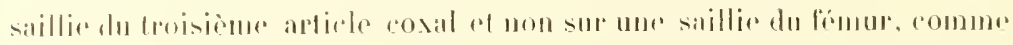

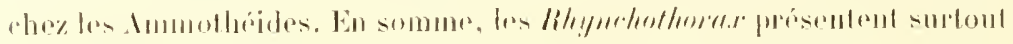

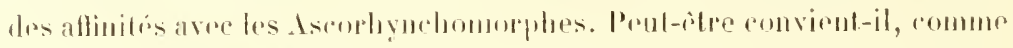

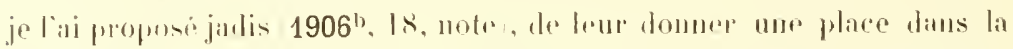

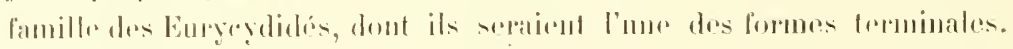

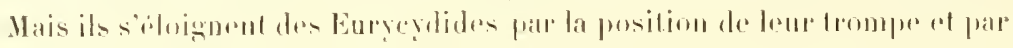

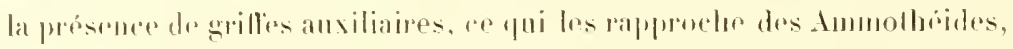

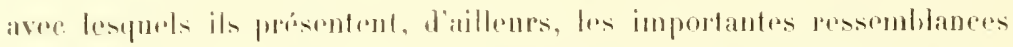

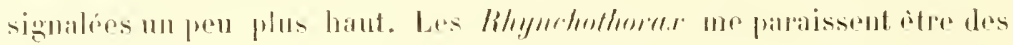

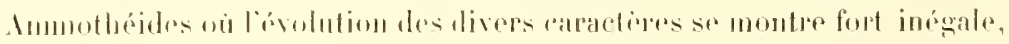

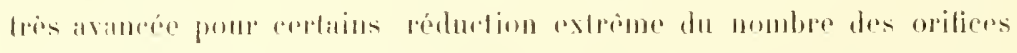

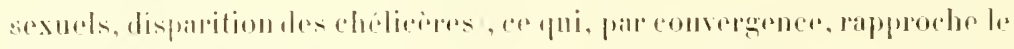

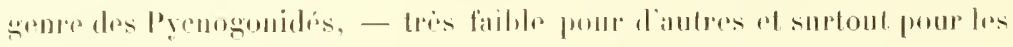

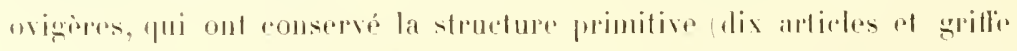

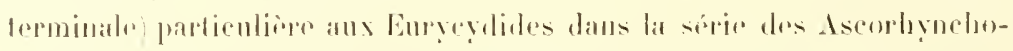

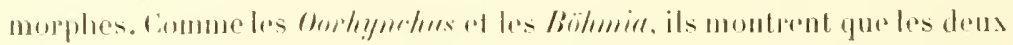

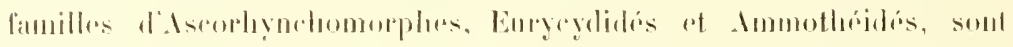

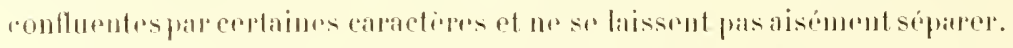

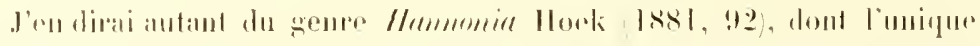

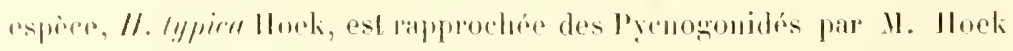

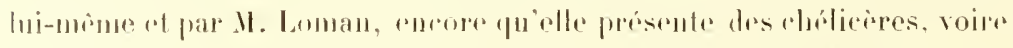
des creficeres dout le scape aurait deus articles d'apròs M. Mrek, l'un

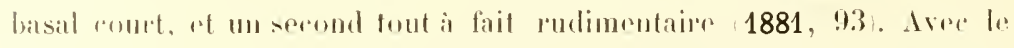

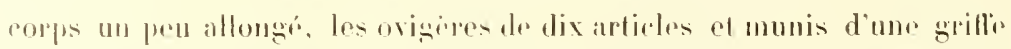

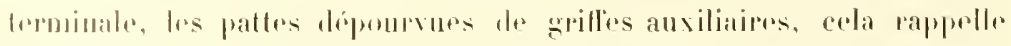

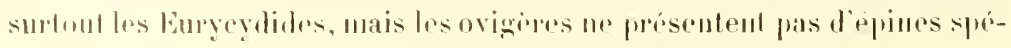




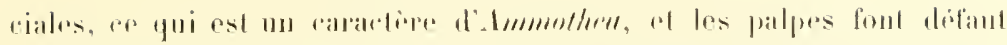

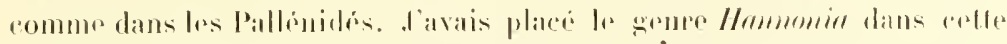

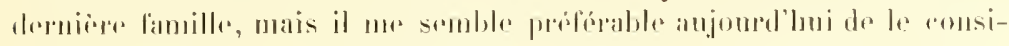

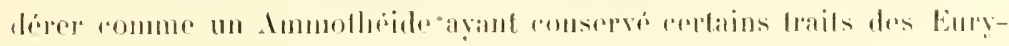
tydides.

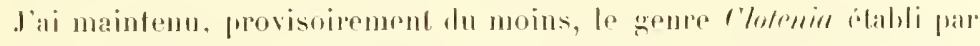

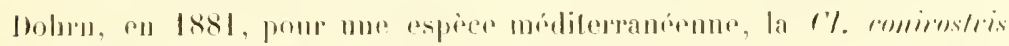

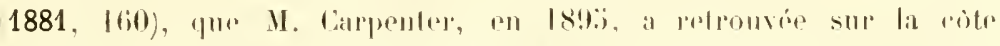

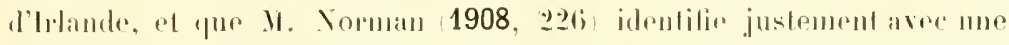

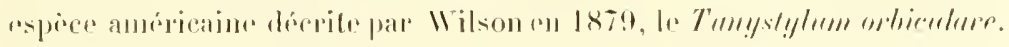

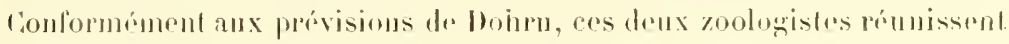

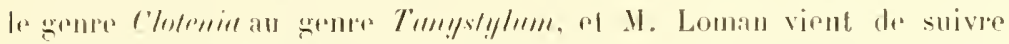

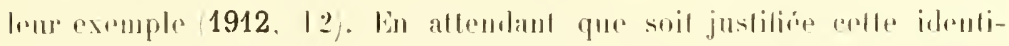

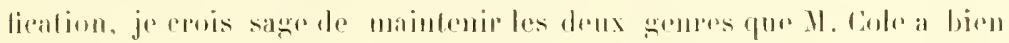
raracterises dans me elude pecente 1904, 277, 280 et qui se distin-

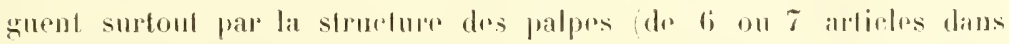

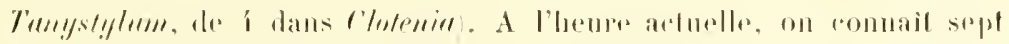

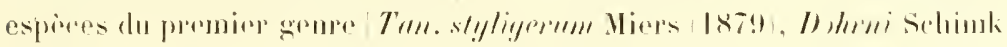

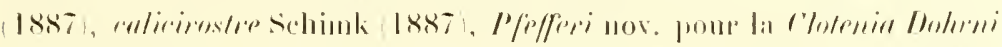

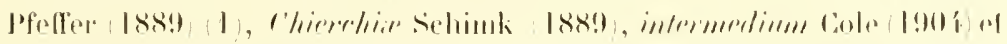

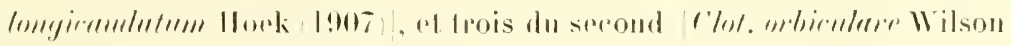

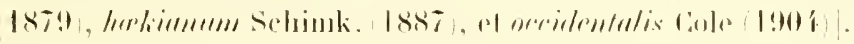

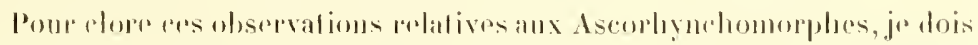

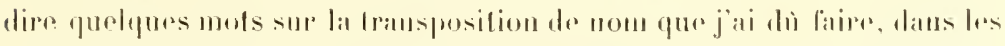

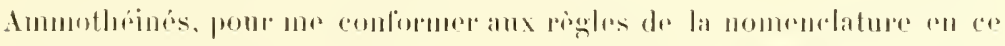

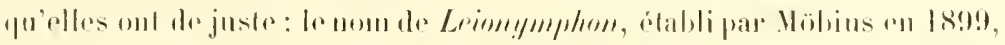

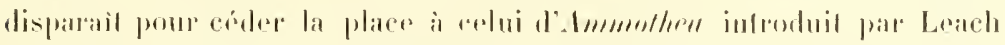
יIs

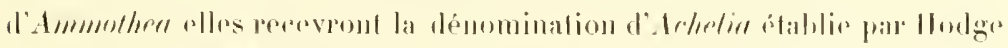

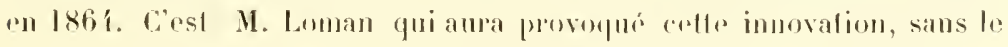
vonloir d'ailleurs, car il est loin davoir une adurimtion sans bornes pour

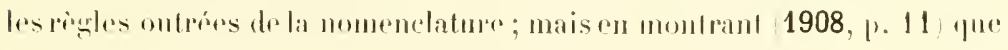

\footnotetext{
(1) Inig plus haut, p. \$.
} 


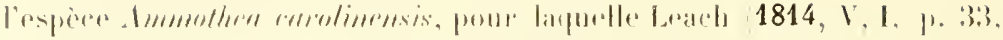

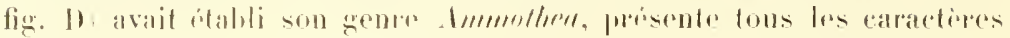

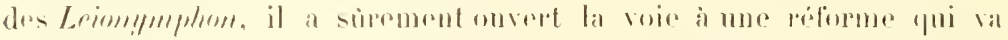

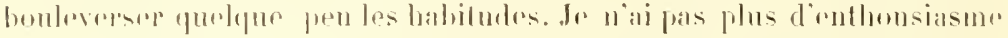

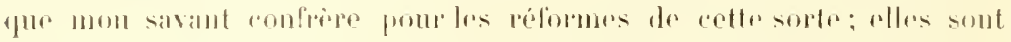

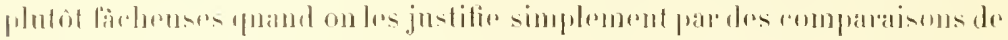

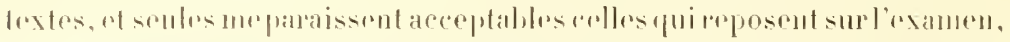

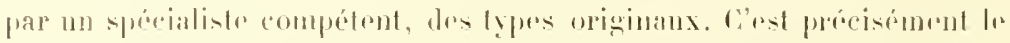

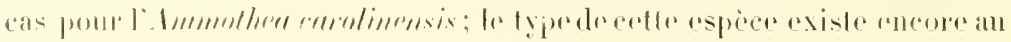

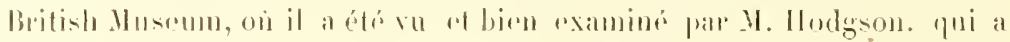

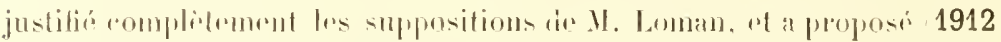

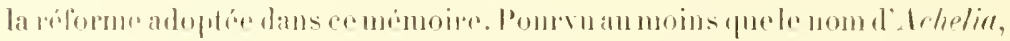

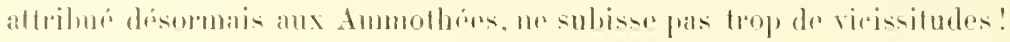

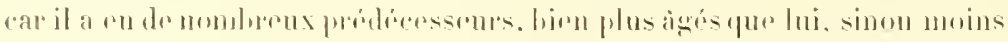

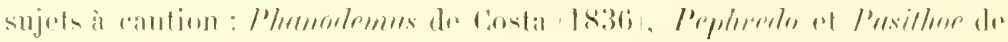

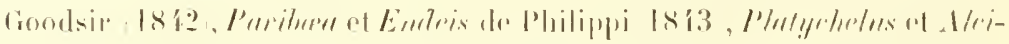

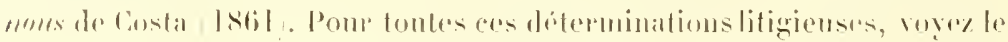

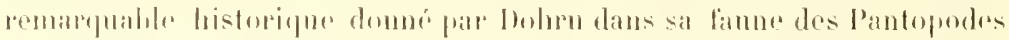
le Xaplos 1881, 227-2:39!.

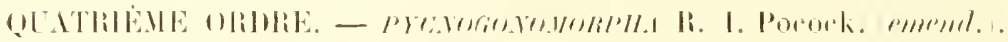

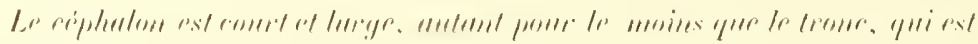

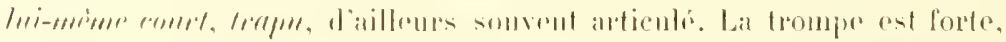

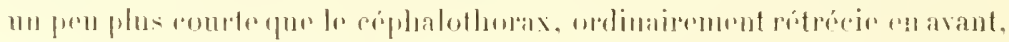

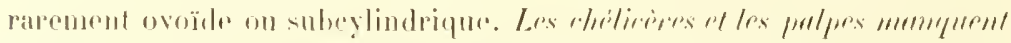

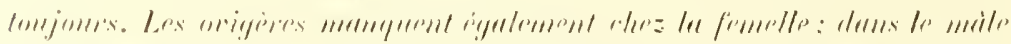

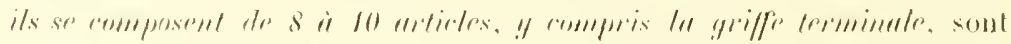

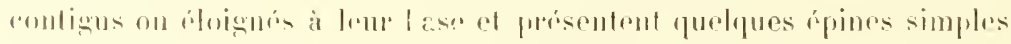

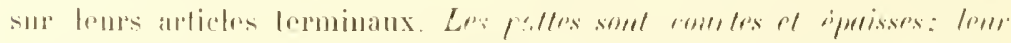

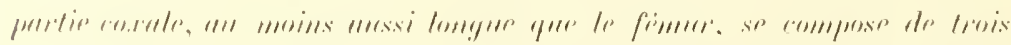

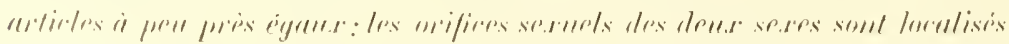

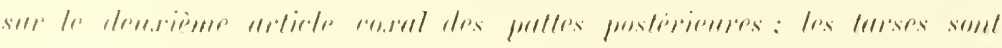

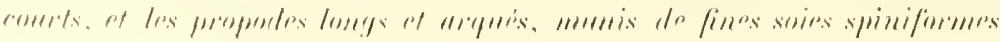


sur len bord interne: les grifles auxiliaires font greméralement defaul. La taille est médioero ou pretile.

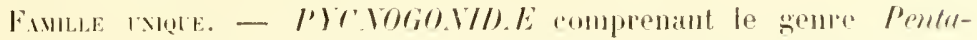

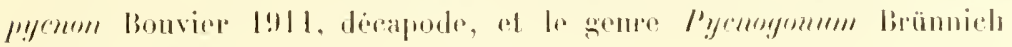
I7ít. octopoile.

(1) peut expriner comme il suil la classiluation or les alfinités des Pyenogunides.

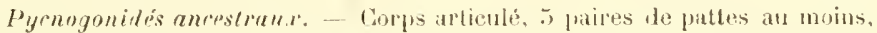

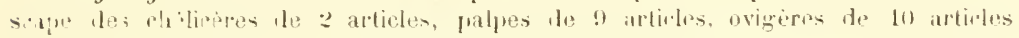
a sines et priffe terminale.
fer orilie:
ze orile:
:3e ordre:
48 orilre :

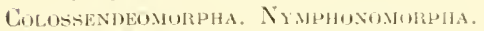

Jecolopodidie.

Type ancestral derapoule.

loblossendeida.

$$
\begin{aligned}
& \text { rentanymplosn } \\
& \text { (diciujule) } \\
& \text { ol . Vymplomidir } \\
& \text { (molopinles). }
\end{aligned}
$$

H

Inllenidar. Inorichilidiular

Euryrydille.

I mmotheidin.
Pentapycnon.

I'yrnoyonum.

Ihorichilidir.

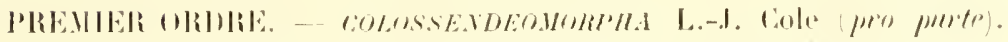

Premiere Fimille. - DECOLOPODID王 1...J. (inlt: (mo prarte).

Genre Decolopoda kights.

La lamille des becolopodidis se limite actucllement an seul gemere

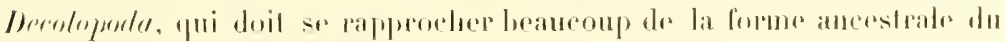

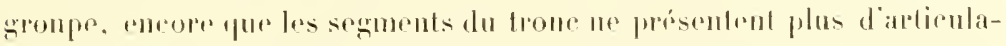
lions mobiles, ni meme, parfois, des traces de lighes anticulitires. Le

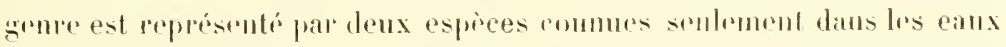

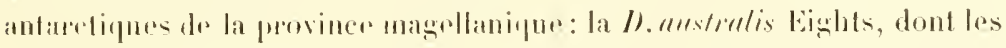

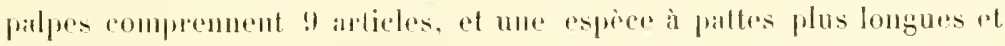

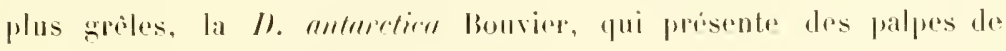
8 articles. Les naturalistes du "l'ourepuoi l'as". " n'ont rapporte que lat premiere de cess deux espieres. 
Decolopoda australis J. Eights.

183i. Hecolopadu australis J. Wighto 1834, p. 21:-29ki, I1. VII.

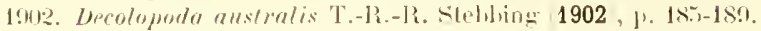

1005. Decolopoda australis .J.-C.-C. Loman 1905, 1. 72:-723.

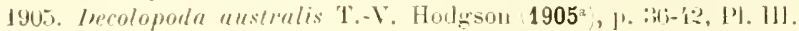

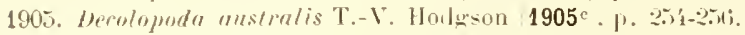

1905. Decotopodu arstralis L..-d. Cole 1905, [1. 40.5.

190k, Decoloperda anstralis G.-H. Carpenter 1905, P.

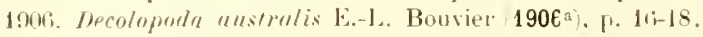

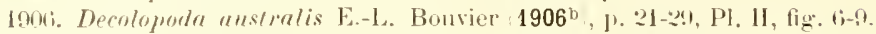

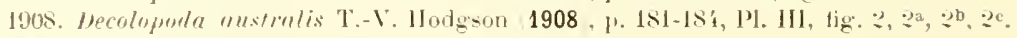

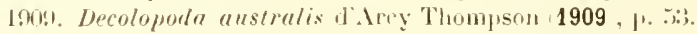

1906. Decoloporta anstralis W.-T. Calman (1909.

1910. Herolopuda australis E.-L. Bouvier 1910a), 1. 27.

1911. Decoloperla australis E.-L. Bousier $1911^{\mathrm{b}}, 1$ 1. 1136 .

1911. Jecolopodu anstrulis E.-L. Bonvier $\left(1911^{\mathrm{c}} \%\right.$, W. 1.

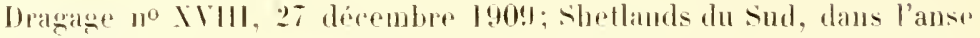

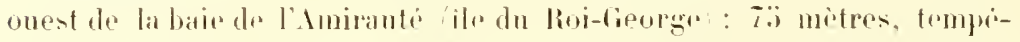

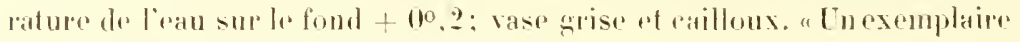
d'un tresbean ronge oranger p $11^{\circ} 73 x$.

Lexemplaime pécédent ust une femella. comme le type unique de la

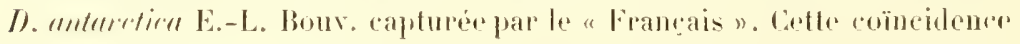
ref lempense, rar ("n donnant les movens de comparer des individus de"

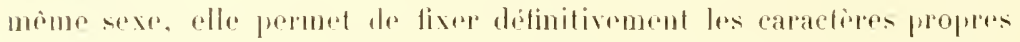
des denx especen. La liste des caracteres distinctils que jai donnere

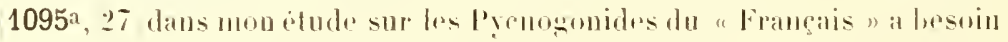

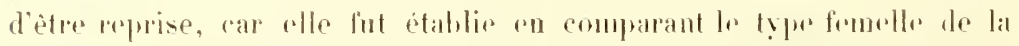

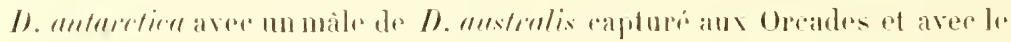

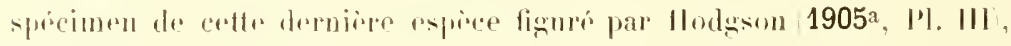

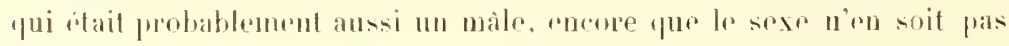
indique:

Alin dre disentrer comme il comvient les cararteres des deus repeeces, jail reuni dans un mome talulean les dimensions principales releveres dams

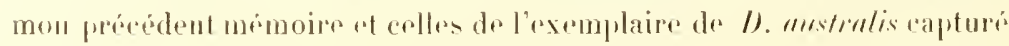
far l" "Pourquoi Pas?" 


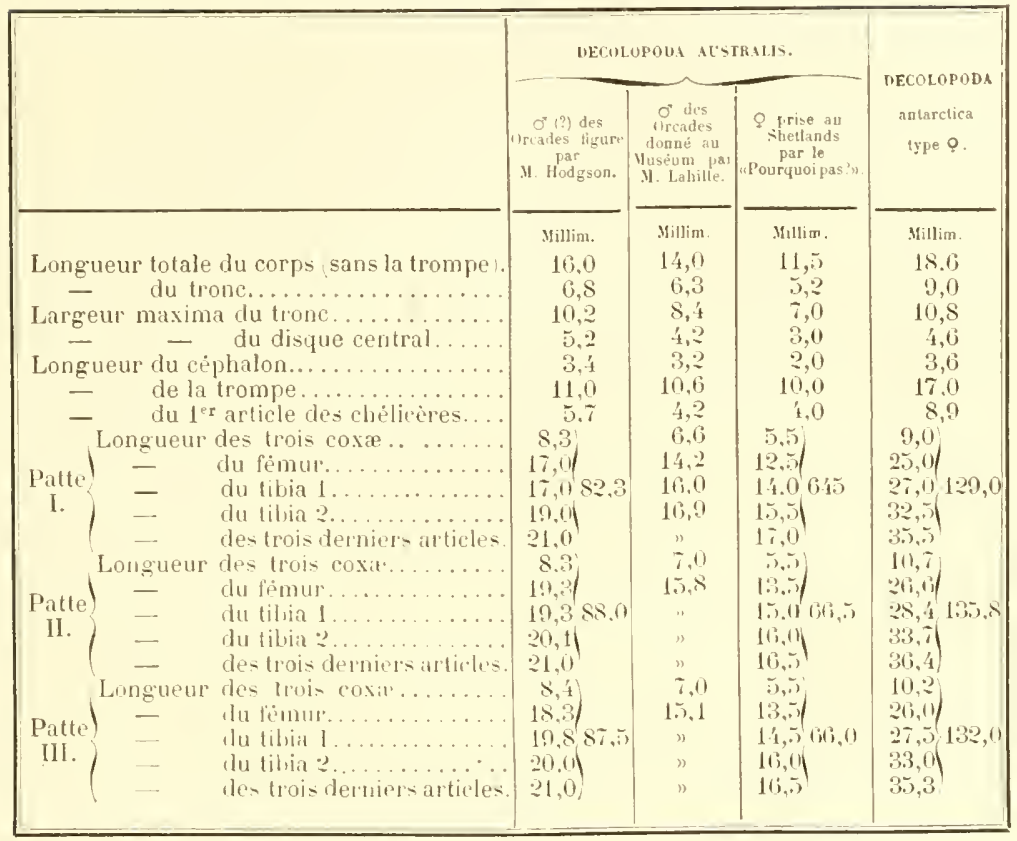

D'après les dimensions relevés dans ce tableau, il semble que la forme du trone soit un earactipe de nalure sexuelle. En ellet. Ie rapport do la longueur à la largeur du trone est de 0.83 daus la DereoIopocla austrulis or. de $0 . i$ i dans la $q$ de meme espece tig. I of de 0 ,iti dans la $q$ de $D$. antureticu. Ainsi, cherz les bécolopodes, le trone serait plus étroit et moins discoüde chez les femelles que chez les miles. Mais avant de poser "ul règle générique la diflérence précédente, il comiembar de vérifier le fait sur d'autres /). australis et d'attendre la déeouverte du mile de la /). anturetien.

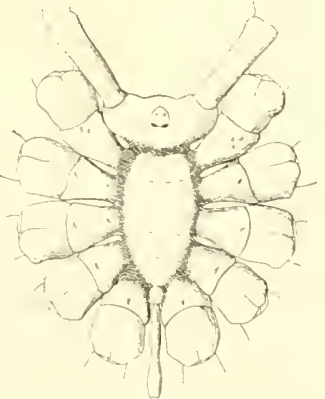

Fir. 1. - Decolopodu australis Eighls, du a l'ourquoi pas? a. - Le cotps el la parlie basato des apponulices rus de hos.Gr. ;

Abstraction faite de ce caractère, les différences qui distinguent la Expedition Churcol. - Bolver. - Prenogonides du "Pourquoi Pas?". 
D. austrntix de la D. anturctica sont exactement celles relevées dans mon précédent travail, à saroir :

$1^{\circ}$ La structure des palpes qui ont neuf articles au lieu de huit;

2o Le dŕveloppement beaucoup plus réduit des paltes, celles de la demxieme paire rgalant huit is neuf fois la largem maxima du trone dans la I). australis, au lien de douze fois commo dans la I). antarcticn:

$3^{\circ}$ La brièreté et l'épaisseur plus grandes du premier article des

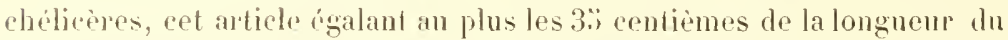
trone et étant à peu pris trois fois aussi long que large; tandis que dans la I). antarctica il mesure les is centièmes de la longuenr du lrone of sa longueur égale six lois sa largeur ;

10 La forme des pinces des chélicères dontla portion palmaire est courte dans la I). anstrulis, on elle se termine jar des doigts fortement inflechis ('n areeau demi-cirentaire, tandis que chez la II). anturetira la portion palmaire est assez longu of so temine par tes doigts médiocrement arqués;

"30 te développement de la trompe, qui est bien plus courte (les 73 -8. centirmes de la longmen du corps au lieu de 9l centiemes) et notablemont phus eitroite dams la ID. australis:

Go Le faible déreloppenent du lubereule oculaire qui est bien plus atroit yno la moitio du céphaton, tandis quil est plus large dans la D. antarerticen.

Ja laisse de cote les caractires moins importants on quelque peu villialins, fels que la longuemr du ze article tibial ol les soies spiniformes du trone, des palles ot de la trompe; dans la I). australis du "Pourquoi Pas? ") ces dernieres sont particulierement peu nombreuses sur le trone romme sur la lrompe, beanconp moins nombreuses que dans l'exem

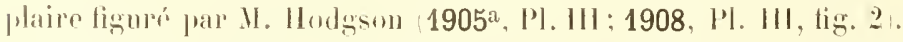

Fant-il considárer comme un caractione sprópifipue la forme des orifices sexucls femelles? Je ne saurais le dire, fante de materiel sullisant. Mais. fomme on le verra dans les figures jointes à re mémoire. les orifices sont ovales dans l'exemplaire de I). anstralis fig. 2, 3, if ef subtriangu-

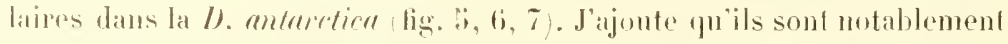

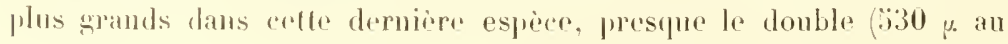


lien de 270 , mais ces diflérences sont peut-être dues il la taille des specimens.

Lacoloration pourraitbien citre un carartipe plus inportant. Elle est tris

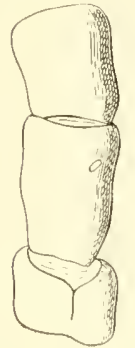

Figr. - Decolopoda australis, meme $\&$. - Mrifiece sexuel de la lie patte gauche. Gr. 8 .

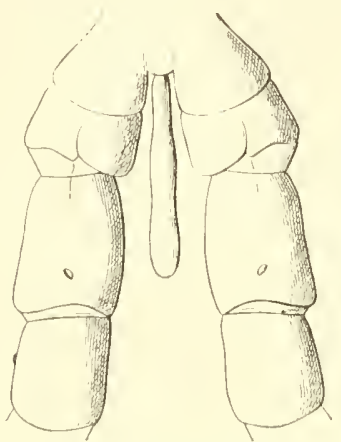

Figr. 3. - Vecolopoda australis, mene? - Ablomen et coxir thes palles poslivicures, face ventrale avec urifice sexuel. Gr. 8. varialite et probablement earactóristique des Algueson se limut l'animal ahem la l). ans/ralis: "Quelıues exemplaires, dil M. Hod-

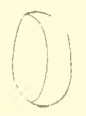

Figr, 1 - Discolopoder austretlis, mine o. - Orofice sexuel de la palte postévicure gauche. Gr. 46.

sson (1908, 183), sont de coulenr paille très légère, sans aucuns trace de pigment, sauf dans un on deux cas ou l'ou trouvail un peu de cehni-ci à lextrémité de la trompe. D'autres exemplaires sout d'une riche conleur brun-olive, ‘ui est considérablement plus foncée, presque noire, sur la

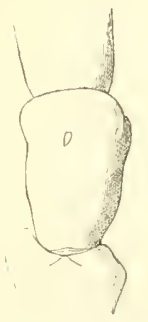

Fig. 5. - Decolopock an larclica Bouv. - orifice sexuel de la Ire palle gauche. Gr. i.

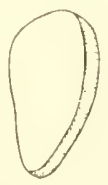

Fig. 6. - Hecolopola an- Fig. 7. - frecolopodit turctica. - Orifice anturctica. - Orifice sexuel de la tre patle sexuch de la je palle gaucle. Gr. 16.

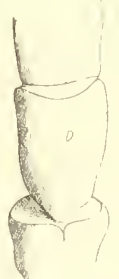

gauche. $\mathrm{Gr}$. i. trompe, les man-

dibules (chélicères) el les palpes. Tans un spécimen, les pattes étaient également très foncées. Les notes de coulent qui unont alfi lransmises et qui furent relevées an moment de la caffure montrent yur certains exemplaires claient d'une brillante couterr écarlale, eomme l'a décrit Eights; d'autres étaient d’un rouge très sombre, et alors, avec la 
trompe prestue noire. Lil couleur écarlateparail uniformément distribuée sur le trone etles membres, la trompe et les parties adjacentes étant plus loncées fue le reste. "Comme on le voit d'apres ce passage, la couleur varie notablement of parait assez fugace dans l'aleool; l'exemplaire du "Pourquoi Pas? s atait d'une befle couleur rouge au moment où il fut capturé, et sa décolopution rest complite a thempe acturlla.

Au contraire, la D. antarticn ast d'une teinte brun clair légerement

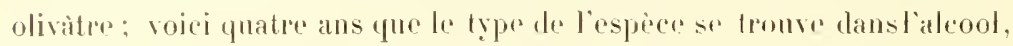
ot sat colorition n'a pas varié. On peut done croire que la D. anterefted se distingue de la D) arstrulis par l'insolubilité de son tégument dams la liqueur alcoolique.

Les deux especes n’ont pas identigurment la mêmm distribution géogriphique. Toutes deux appartiennent a la province magellanique tell.

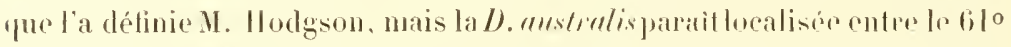
Oreades du sud et te b30 Shetlands de latitude sud, tandis que la l). an-

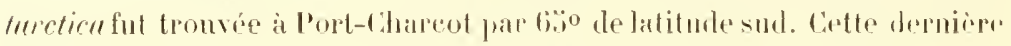
espece parait done phus franchement antaretique que la précédente.

\section{Deuxième Famille. COLOSSENDEIDA P. P. C. llake.}

Cette limille se distingue de la précédente par sa structure octopode et par la disparition des chélicipes; elle comprend les genres Colossendeis Jarz. Rhepalorhynchus Wood-llason ef Pipelln Loman. Les deux premiers genres sont des Colossendromorphes absolument norman, eneore que, d'apris II. Loman (1908, Ill. XV, tig. 2l:̈), les palpes semblent doignés des ovigiones dans less Rhopulorhynchus; le gemo l'ipothe est un peru abrirant en cresens que le 2e articlo coxat des pattes est trìs allongé au lien d'avoir la mème longueur que les deux articles contigus. Il ne sturait être question de faire dériver, avec II. Cole $(1905$, 410), les membres de cette fimille des Ammothéidés. Ainsi que l'ont montré M. Ilodgson (190, 41) et M. Lomin (1908, 1:i), Jes Colossendeis se rattachent étroitement anx Decoloprula, of le second de ces auteurs range même le genere Decolopmla dans la famille qui nous oceupe.

Lomme je l'ai fait observer antérienrement $1906^{b}$, l:i), le genre clonteux Pasithoe Goodsir ne rentre pas dans cette fanille et doit prendre 


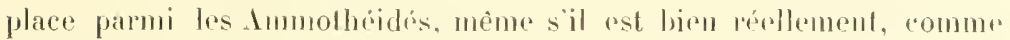
l'a dit Goodsir, dépourve de chéliciores Voir p. 1:3!), ee qui le rappro-

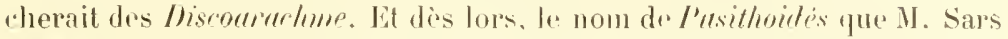
et M. Cole attribuent aux Cotossendeidés doit disparaitre de la nomenclature zoologicue.

Le genpelhopalorhynrhus astrepresentipardenx especes sublittorales localisées dans les mers indo-pacilipues of hogenre l'ipettre par la P. Iteheri Loman, eaplurep ou sud d Amboine, a 2081 miotes de profondeur. par la "Silogan. Les tolessendeis ont au contratre, on to wera plus toin, une distribution fort vaste, et representent sentus la famille lans lex eaux antarctipues.

\section{Geme Colossendeis Jarzunsky.}

Les Colessembers sont vaisrmblablemont issues des Derolopoda ; la plupart, il est vai. ont un rorps allonge ot plus ou moins supêle, lort

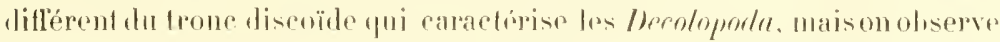

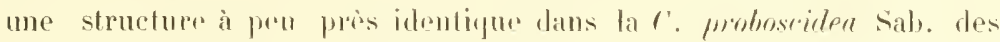

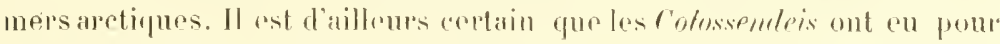

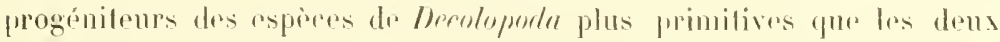

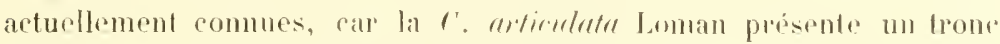

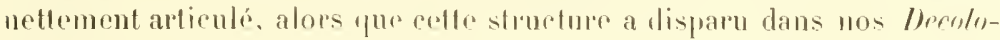
jodil.

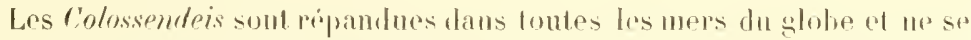
plaisent qu en eautroide, de sorle pu'elles sont abyssalres dans les rógions chaudes et peurent devenir littorales ou sublittorales à mesure qu'ou so rapproche des pòles. Leur taille est médiocre on grande, parfois lres grande; e'est parmi elles qu'on troure les représentanls les flus volumineux de l'ordir.

Le genre est acluellement représmotr par 28 especes, yui peuvent se répartir en deux groupes, les lmogiturses et les hreviturses; dans le premier groupe, les trois derniers alliches des pattes pris rosemble igalent an moins les trois quarts du sacond lihia ol souveut mème dépassent en longueur cet article; dans te second, ils prósentent loujours une longueur beaucoup plus faible. 


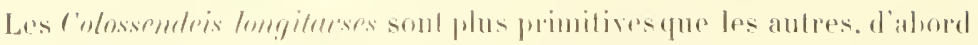

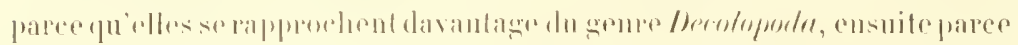
y

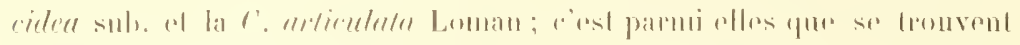

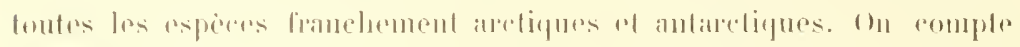

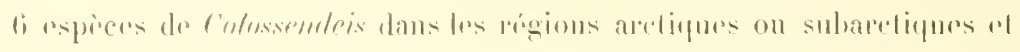

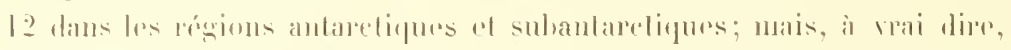

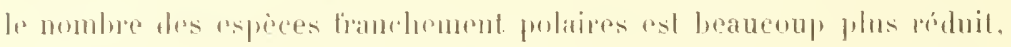

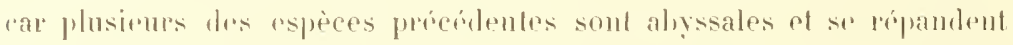

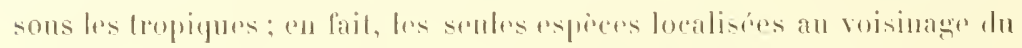

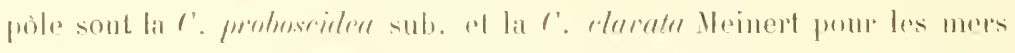

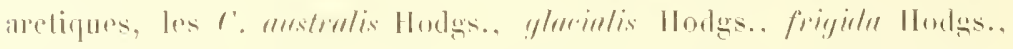

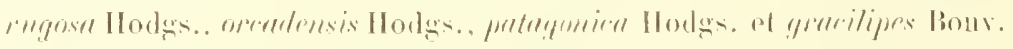

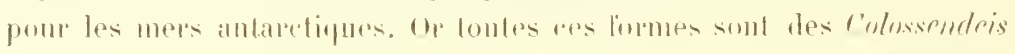
longitarses; les anteres amynstn sars, rolossme Wils., maremimu Wils.

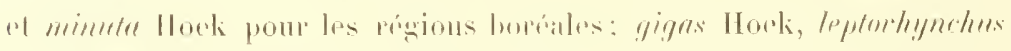

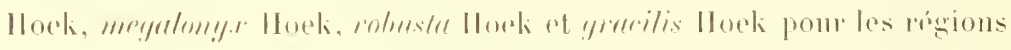

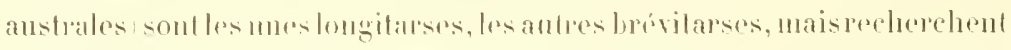

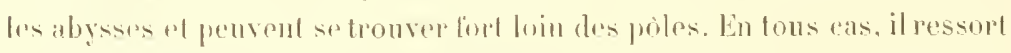
de re gui precide que les colosspmleis sont heaucoup plus mombreuses

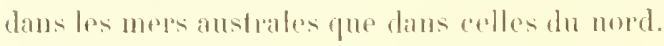

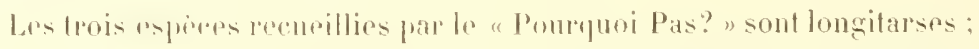

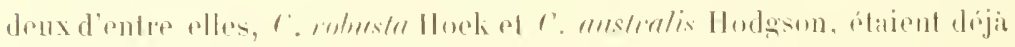

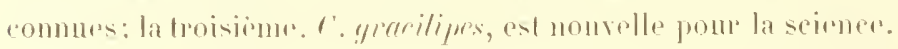

\section{Colossendeis robusta Hoek.}

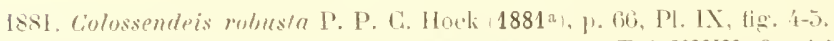
190?. Colossendeis robust" R. Mobius 1902, 1. 19!, Taf. XXIX, fig. 1-5. 1011. Colosiendeis robusta E.-L. Bouvier 1911 b, P. 11:5i.

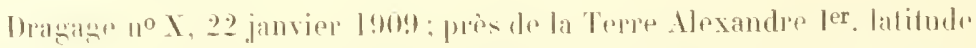

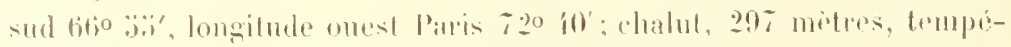

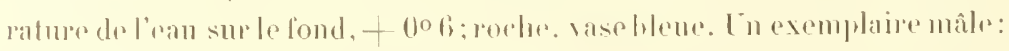

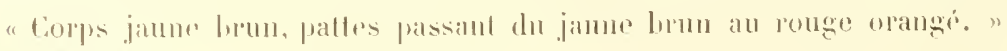
No 280 . 
L'exemplaire précedent dilfore du lype unique de liespece pal les caractères suivants:

Jo Sa trompe lig. 8 at y) est notablement plus allongere et plus btroite, arec les dilatitions dı milieu el du bout moins acernturios; elle est plus courte pror

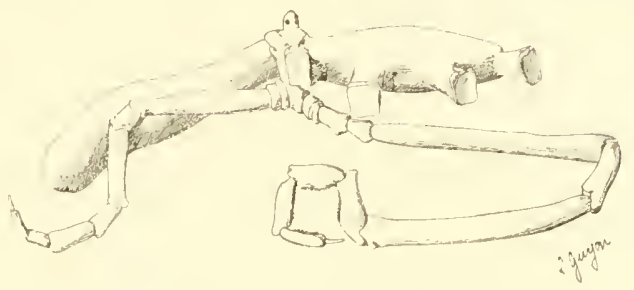

Fig. X. - tulossmistes robusta o. - Corps du cioti gauche itver le pallun ret l'ovigire. Gr. 2. le tronc dans la figmere domne par It. Hoek, un prou phus longue daus notie esemplaire, ainsi qu'on le verra dans le labtean comparatil denne ai-dessous;

20 Son Luberentr oenlatire

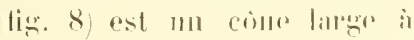
sommet lais oldus ol meme anrondi, tandis qu'il est en forme de còne étroit et tris aign dans la figure i du mémoire de H. Iloek;

30 bins lo lypede II. II ocek, le finmur el le tibia sont dre mème longuenr et notablement plus lougs que le libia I: te fémur seuble un pen plus large que te trone dans l'blranglement qui scipare les paltes 2 al 3; ro outre, lis grillo terminale igale environ la moitioi da la longuent du propode; dans notre exmplaire, pall contere, le lémur

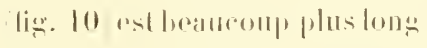

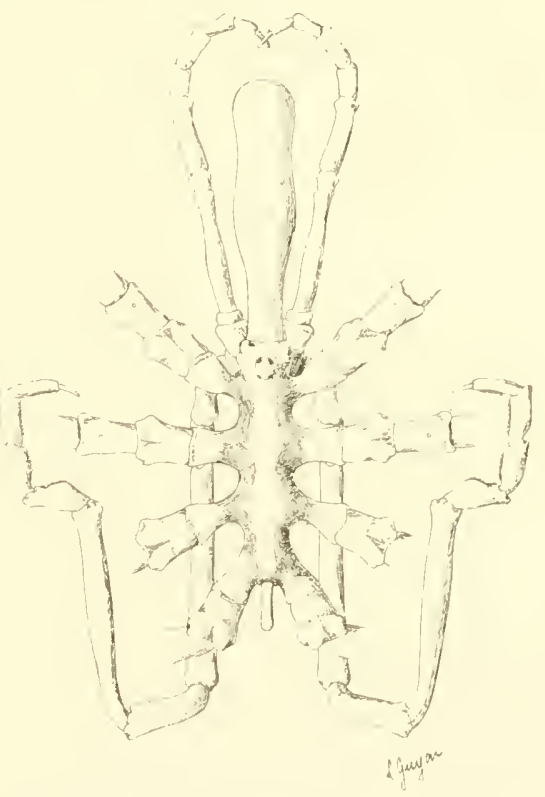

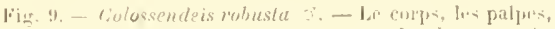

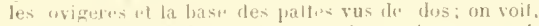

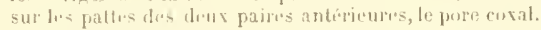
(ir. 2. fure le premier libia el lisgi-

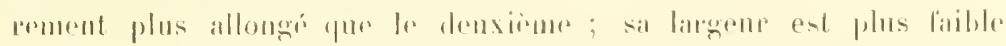


que celle du trone dans lebtranglement signale, et la griffo fige. Il est notablement plus longue que la moitic du propode.

fo Labdomen est pus long et, an lien de s'attrinuer d'avant en arrière,

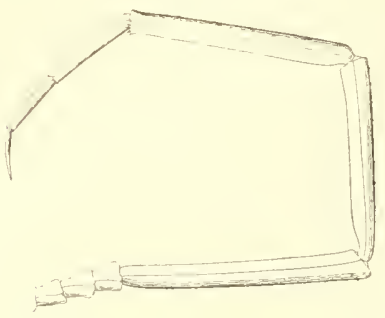

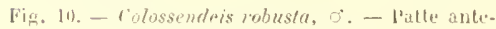
rieure druite. Gr. nat. se dilate un pern ril massue (fig. 9).

Le tableau suivant donne les di-

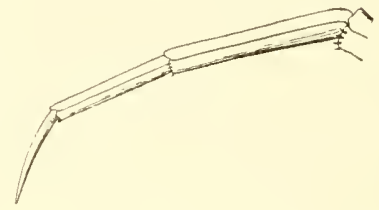

Fin.11. - rolosiendeis robusta. - Les Irois artieles terminaux the la patte antericure droite. Gr. a.

mensions des diverses parlies de l'animal, dans le type du "Challenger",

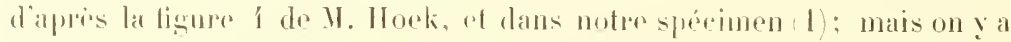

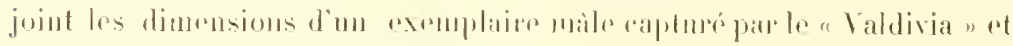
attribur par Däbius à la meme respoee.

Longueur de la trompe........... . . . .

Diamètre maximum de la tromple...

Longueur du céphalohurax . . . . . . . . . . .

Largeur du même entre les paltes 2 et $3 . . .$.

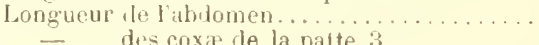

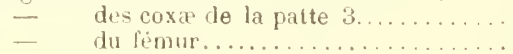

- du tibia $1 \ldots \ldots \ldots \ldots \ldots \ldots \ldots \ldots \ldots$

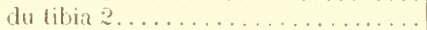

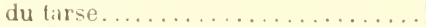

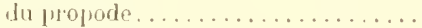

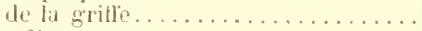

Lareur du femur. . . . . . . . . . . . . . .

Jongueur des roxie de la patte $2 . . . . . . . .$.

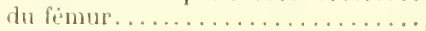

du tibia $1 \ldots \ldots \ldots \ldots \ldots \ldots \ldots \ldots \ldots$

du tibia $2 \ldots \ldots \ldots \ldots \ldots \ldots \ldots . \ldots \ldots$

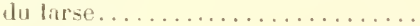

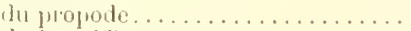

de la grille

\begin{tabular}{|c|c|c|}
\hline $\begin{array}{c}\text { Type } \sigma^{4} \\
\text { du } \\
\text { a challenger: } n .\end{array}$ & $\begin{array}{c}\text { Type } 5 \\
\text { du } \\
\text { "Puurquoi Pas?n. }\end{array}$ & $\begin{array}{c}\text { Type o } \\
\text { de la } \\
\text { "Valdivia } .\end{array}$ \\
\hline 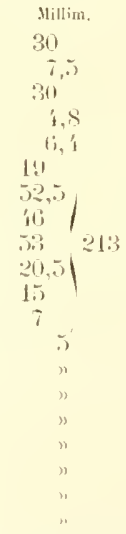 & 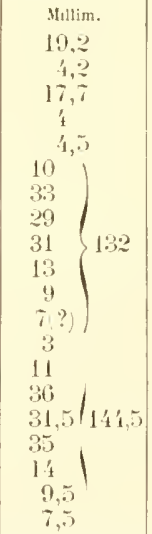 & $\begin{array}{l}\text { Millim. } \\
17 \\
10 \\
10 \\
17\end{array}$ \\
\hline
\end{tabular}

1. Dans le type lu " thallenger ", la seule patle complitement ligurée ast la lroisième du côlé sauche. Yais. dans nolre exemplaire, cette patte fut brisée et se présente à l'ètat de bourgeon repoussant, de sorte quill a élé nécessaire de relever les dimensions sur la palte du coté droit. 
Certaines des dillixences précidentes, notamment la plus grande largerur du trone et la graeilité relative des pirtes, sont peut-che thes an sexe, mais la plupart correspondent à des variations individnelles. Car notre exemplaire appartient trìs certainenent a l'espèce du "Challenger "; il en a totalement le facirs et, abstraction faite des dillérences précédentrs. il rópond jusquau détail a la description de M. Hork.

On est en droit de se demander s’il en est de même du l'yenogonide

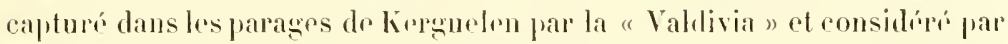
Möhius comme une lol. rolmestu. Haprès les dimensions relevées par Möbius, il pessemble beancoup plus a notre spécimen yu’ì celui du "Challenger ", et commo il est figuro avo un tronc fort large, ales pattes pelativenent ggrêles, il y a dos raisons de penser que réellement ces deux derniers caraetères sont de nature sexuelle dans lesprice. Par mallıeur, il est difficile d'ètre allirmatif sur ce point, car, avee son tronc démesurément large, ses prolongements latéraux à peinc écartés, ses hanches sans dilatation teminale el ses griltes presque aussi longues que lo propode, la figure densemble (1902, fig. I) donnre par Möbius derrait lorl justement ètre rapportíe à une antre espèce. Nais cetle lìnure fut exécutép à coup sù sans grand souci de l'exactitude (trompe el abdomen dropourvus de l'articulation basilaire, artieles des pattes de longueurs dissemblables), ef laissée, on doit le croire, à la lantaisie du dessinateur. Une fois celte comviclion açuise, on recomnait à certains détails forme de la lrompe, grossenr despalpes) l'espéer du "Challenger ", et les figures de détail données par Möbius 1902, lig. 2, 3, 4, ; vicnnent fortilierectte conviction.

Je dois ajouter que, dans la tigurr I de Möbius. lr dessinatene a dì singulierement exagrárer les lignes arliculaires des segzments du trone. Ces lignes ne sunt ni ligurées, ni représentées par M. Hoek; elles existent tres nettement dans l'exemplaipe du "Pourfuoj l'as? ", où wlles se voient surtout bien quand l'reaporation a fait dispanatre des tignnents l'aleoul superficiel. Ces lizgnes me sont nullement indiqures par un sillon articulaire, commo on pomrait le pronser d'apres la ligure I de Möbius: alles résultent simplement de la dillórenciation du revêtement cutaní, qui, en ces points, reste clair, au linu de presenter, comme partout ailleurs, de minusculos taches. Ces derniores paraissent jamaitres Expédition Charrot. - Bouvien. - Pyenogonides du "Pouripui P'as?". 
dans lixemplaire dreolorí que nous avons sous les yeux, mais elles sont, à m'en pas douter, des centres de formations pignentaires.

Qnoi qüil en snit, l'exmplaire du "Pourquoi Pas?" permet de rattacher sans conteste (il me semble) l'exemplaire ćtudié par Möbius à celui décrit par MI. Hoek soms le nom de C. robusta.

Cirs trois cxemplaires sont, it theure actuelle, les seuls reprisentants

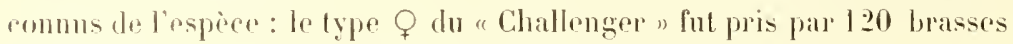
de profondeur. an large de Christmas IIarbour, iles Kerguelen; — c'est ŕmalement aux kerwneten, par 88 mitres, avee une température de fond do $4^{\circ}$, que fut pris l'exemplaire ơ de lis "Valdivia ".

Ainsi, avant la campagne du "l'ourpuoi Pas?", l'espèce ćtait tenue pour subantinetique ef étroitement localisée. Anjourd'hui, on doit la considerer aussi comme antarchipue of certainement circumpolaire, car la Terre Alexandro Jer, où M. Gian captura notrespreimen mâle, se trouve presifue à l'opposí des Kerguelen (par 720 longitudr ouest au lieu de (i folongitude est), et la température du fond où vivait l'animal ue s'éleviit pas au-dessus de $+0^{\circ}, 6$.

La coloration sur le vivant itait d'un "beau rouge orangé " dans le type. "rouge-minimu dans lexemplaire de la "Valdivia ", "jaune brun " sur le corps avec les "pattes passant du jaune brun au jaune orangés dans notre sprécimen. Il existe done, là aussi, des variations individuelles.

Colossendeis gracilipes E.-L. Bouvier.

1911. Colossendeis gracilipes F.-L. Bonvier (1911ํ), P. 1137.

Iragage no X: 2.2 jauvier 1909! ; pres de la Terre Alexandre-Ier; latilude

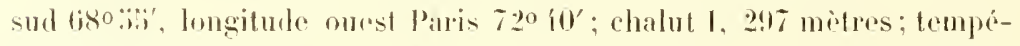
rature de l'eau sur le fond + 110,6 ; roche, vase bleue. - Un mâle adulte "orangén s il l'état vivant ( Yo 281 )

I) ragage no XYIIl : 25 décembre 1:009; Shetlands du Sud, anse onest de la haie de l'Amiratuté dims l'ile du Roi-Georges; chahut I, zij mitres, tromprérature de l'ean sur le foud $+0^{\circ}, 2$; rase grise, cailloux, - Une femelle adulte "d'mblen rougeatten sur le vivant (No 7 il).

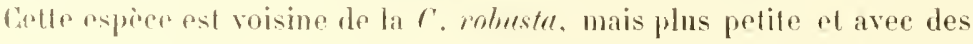


pattes bien plus grèles; chle se rapproche certainement anssi heaucoup de la C. petugonica llodgson $(1907 \mathrm{~b}, 18)$, dont, par matheur, on ne connait pas la taille.

Par la brièveté et le faible élargissement antérieur du céphulon lig. 1:2 et 13 dont le lood antériem est droit, par les dimensions du trone at le srand icartement de ses prolongements latéraux qui sont rétrécis à la base, par la forme de l'abdomen qui se dilate un peu en massue dans sa partie terminale, notre espéce ressemble complètemont à lat f'. mbustu; le lubercule oculaire est également identique dans les denxespices, un peu plus large pourtant dans la nôtre, où il enrahit ume plus srande partic du céphalon; audessus des yeux, qui sont noirs et partaitement limités, le Lu-

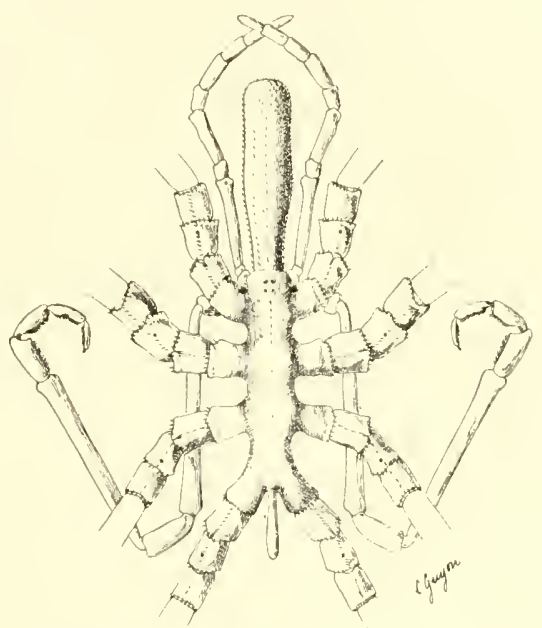

Fir. 12. - Colossendeis gracilipes Bouv., ९. - Corps aver las palpes. les ovigires et la base des fraltes, lince dortide. Gi, i.

bereule se terming par un cone aigu très rógulier. Comme dans la r. robustu, les yeux de la face antérieure sont beaucoup plus grands tue ceux de derriere. La fare dorsale du ciphaton est déprourvue du monticule oblus que jai observó, a droite et à ganche, près des angles latéraux antérieurs dans notre spécimen de f'. rohneta. Toutes ces parties du corps sont unies et sans poils, sauf yucliues tres courtes soies distribuées sur la lign" médiane dorsale, dams l'exmplane male. Les lignes intersegmentaires sont efficées completenent dans la femelle, al peu sensibles dans le mâle.

Le's soies sont hien phus nombreuses et sensiblement jhus longues sur la trompe (lig. 12 et 13), qui est nue, comme on sait, dans la f'. rohuefu. Ceet

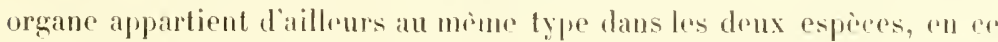
sens qu il se dilate à partir du milieu et de vient ensuite beaucou! phus lares. 
jusquau sommet. Mais, tandis que celte partie dilatée présente, dims la 1'. robusto, un renflement basilaire et un renflement terminal, olle est à peu près cylindrique dans notre espèee, tant ces deux renflements sont peu marqués. Jajoutr que la trompe est prestue droite ef nettement phos courtrune le céphalothorax dans motre especer, tandis quiplle est a pen près inssi large on plus large dans les deux autres especes, ef d'ailleurs

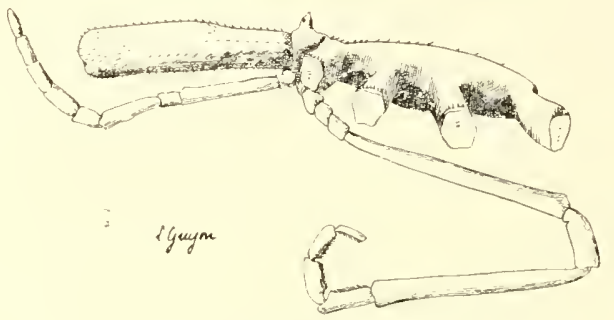

Fir. 13. - Colossendeis gracilipes F. - Le curps du cole sauche aree palpe el ovigere. fir. i. nettement recourbrevers

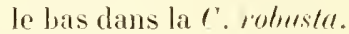

Les palpes (fig. 12 et 13) sont presque identiques à ceus de la $C^{\prime}$. rolinsta, mais richement pourvus de soies raides dans notre espece, tandis qu'elles sont plutiol

tres ratres dins la 6 moluestu. Liaticle terminal est étroit et, an plus de la longueur du précédent chez cette dernière espèce, alors qu il est assery large et notablement pho long que les articles 8 ot 9 dans l'espiece du " Pourquoi l'as?". Au surplus, dans cette espèce, les dimensions relatives des divers articles à partir du denxirme sont exprimées par les nombres $10-2-6,3-2,3-i-2, i-2, i-3,4$. Ces mèmes ripports sont les snivants dans notre exemplaire de $r$. roluestu, oủ les nombres qui les rxpriment représentent des millimetres: $9 ., 3-1,: 3$ $-6,2-2,2-3-2,4-2,3-2,1$. Mans la $C$. palagmica Hodgs, espice émalement trés voisine, les rapports sont exprimés par les nombres $10-1,3-\ddot{3}-3-3,3-1,3-2-3$.

l'ar les dimensions relatives de leurs articles, dont les quatre derniers

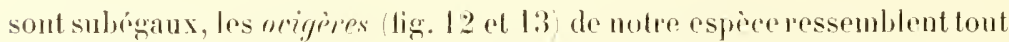
i fait a cenx des deux especes próndentes. Mais la grillo terminale et les epines spéciales sont tont autres, ot dialleurs dillerent considérablement dans le nate et dans li femelle. La grifle du màle (fig. 14 et lia) est bien théveloppére, quoique fort étroite ot égalant au plus la moitie de la longuenr de l'article précident; quant anx épines des quatre articles terminax, alles sont très longues, étroites, obtuses ou subaiguës el sans 
denticulations apparentes sur les hords. Wans la femelle (fig. 16 ef 17) au contraire, la gritte est totalement absente des deux cotis, et les ripines des quatre articles sont tres courtes, arrondies en sommet, comme si plles rtaient usieres et reduites à leur base. Il y all a d'ailleurs trois rangées fort netles dans les deux sexes, comme dans la r. putngonican.

Les pattes (fig. 18 et 19) sont phus grêles que dans la $C$, rolustu; leur límur est à peu pris de mime longuerur que le tibial 1 , beatucoup plus long que le lilia 2. ce qui rappelle ainsi la

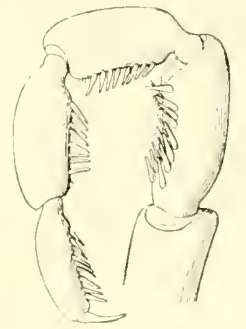

Fig. 1t. - Colossenrleis ynacilipes, $0^{\circ}$ - I'antie terminale de lovirere gauclie. Gir. 10.

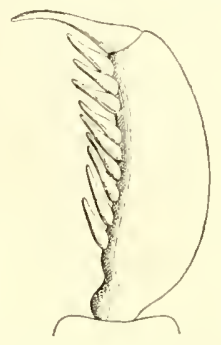

Fing. 15. - Colossendeis fracilipes lious. - Uvnere rauch" du $0^{\circ}$, article terminal et sriffe. fir. 23.

1'. puntagmica, tandis que dans la f'. mohresta c'est le tibia 1 qui est l'article le plus conrt, le tibia 2 et le fémur chant de longueur subégale.

Le doigh est un peu plus long que la moitiri du propode (fig. 19), lequel igate lui-meme le trois quirts du tarse. Les articles coxaux sont courts et subégaux.

L’alidomen tis. 12 égali à peu pròs en longueur le dernier segment du trone: il est légìrement recourbí te haut en bas et dilaté en massue dans son tiers postérieur.

Les paltes sont munies de courtes soies spiniformes disposíes en rangies

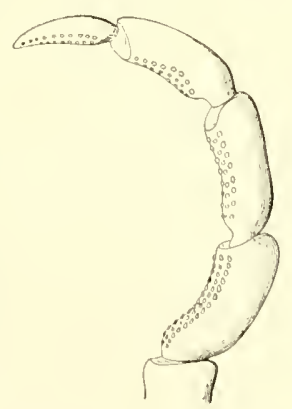

Fig. I6. - colossendeis gracilipes, - Parlic termiralle de liovicines sauche. tui est dejourvu de gatitle. (ir. 10.

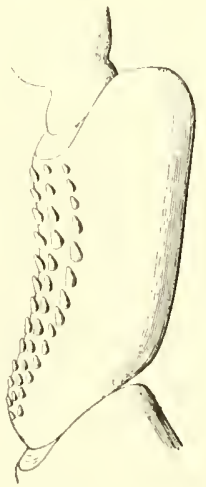

Pig. 17. - Colossendeis gracilipes, o. - Iminle termi nal des wignes aree ses tounes. Gr. longitudinales (fig. 18, 19). Les rangrées principales sont an nombre de trois au-dessus at au-dessous des lignes laterales. Entre ces rangros essenticlles on trouve d'autres saillies spinifïres moins régulierement 
disposies, surtout chez la femelle, ou elles sont particulierenent nombreuses et ahondintes.

Les très pelits orifices génitaux du màle occupent leur position ven-

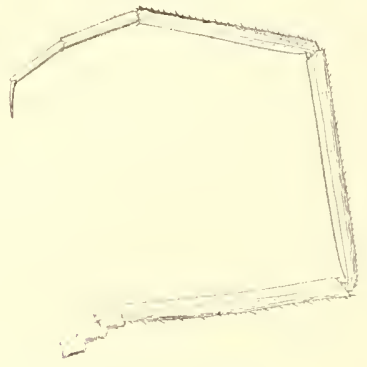

Fig. 18. - Calossmdeis gracilipes, 07. - Tne patte. liv. 2. frate ordinaire, près du hord distal un peu relevé du deuxiome article coxal. Dans la femelle, ou l'orifice est beatcoup phis srand, il recupe la déclivité proximale d'une assez forte

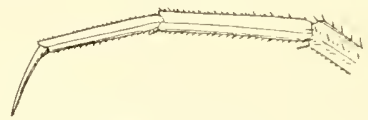

Fig. 14. - Colossendeis grucilipes, o* - Les 3 arlivles leminaux de lit palle de la figure 18 . Gr. 4 .

bosse situé distalement sur le coté ventral du même artirle. Par contre. le pore glandulaire dorsal est beaucoup plus apparent dans le mâle que dans la fenelle, ou d'ailleurs il oceupe la mime plate (lig. 12). sur la ligne médiane, à la maissance du liers terminal de l'article.

Voici les dimensions des deux exemplaires lypes de l'espice :

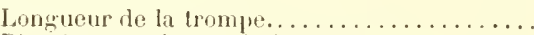

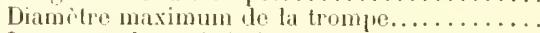

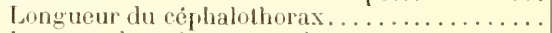

Largeur du meme entre les palles $\because e \mathrm{el} 3 \ldots .$.

Longueur de l'abdomen..................

- des coxre de la palle $3 . . . . . . .$.

- disfimur $\quad \ldots \ldots \ldots \ldots \ldots$

- dil tibia $1 \quad \ldots \ldots \ldots \ldots \ldots$

- du tibia 2

- du tarse

$\cdots \cdots \cdots$

de la griffe $-\quad \ldots \ldots \ldots \ldots$

\begin{tabular}{|c|c|}
\hline 0 & 9 \\
\hline M11lim. & Millim. \\
7 & 8 \\
1,5 & 2 \\
8 & 10 \\
1,5 & 1,8 \\
2,3 & 2,15 \\
4 & 1,7 \\
16 & 18,4 \\
16 & 18,3 \\
$1: 3$ & 14,5 \\
6 & 7 \\
1,5 & 2,7 \\
2,5 & 2,7 \\
1,3 & 1,4 \\
\hline
\end{tabular}

I'apriss les mestures précedentes, les piltes sont relilivement plus

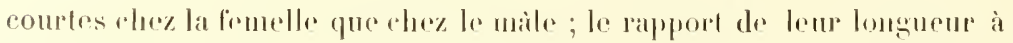

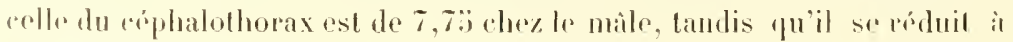
7,03 chez la fenelle. 


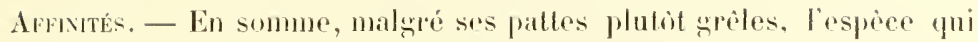
nous oceupe appartient bien au lype des folossendeis longitarses. Commo

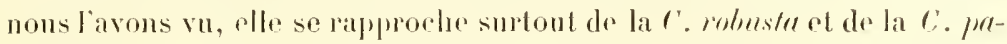
fagmica: mais olle se distingue aisément de la première par ses paltes bien plus gretes, sa trompe droite el plus comble, ses palpes dont le dernier article est plus long qur lo précédent, mais surfont par ses pattes, dont le fémur ot le tibia 1 sont subémax et heancoup plus fongs yme lo libia 2 (te tibia I est l'article le plus court dans la $r$. robustre); elle se distingue de la seconde par la longueur plus grande dn troisieme article des palpes, la réduction retreme des griffes des ovigires et par les dimensions plus réduites du fémme, qui, dans la $C$. patagonica, rst beaucomp plus long que lo libia I; jajoute que le premier article coxal est tres nettement distint do prolongement lateral qui le porte dans notre espere, tandis qüil sen distingur it peine, el senlement par une fine ligne articulaire, dans la $C$. patngomica.

On sait que la $C^{\prime}$. patagnnira fut recueillie par l'expédition magellanique hamhomrgeoise, dans los régions patagoniennes de l'Amŕpique du Sud, sous f fo l í latilude sud ef 61023 ' longitude onest Greenwich, la profondeur étant de fio brasses.

\section{Colossendeis australis.}

1907. Colossendeis australis T. V. Horgson $\left(1907^{\circ}\right)$, 1. 59; P. IX, fig. 1, 1b, $1^{\mathrm{c}}$; PI. X, fig. 1 et 2 .

1911. - - E.-L. Bouvier $\left(1911^{\circ}\right)$, P. J13.7.

Iragage $1^{\circ} \mathrm{IX}$ : 2 I janvier 1909; an sud de l'ile Jenny; latilude sud 68,

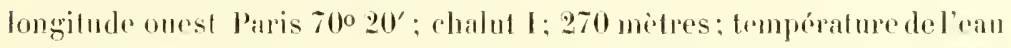

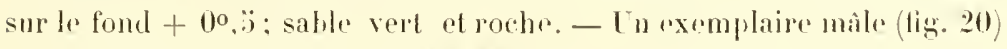
"janne orangze "sur le vivant ( Vo 20"is).

Les dimensions de ce masnilifue exemplaire sont les suivantes:

Lillim.

Longueur de la trompe....................... 35,5

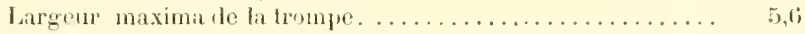

Longueur du tronc.............................

Largeur du trone entre les deuxicue el troisieme paltes..... 4,2

Longueur de labdomen.................... 5,8

Palte droite de la deuxieme paire:

Longueur totale des coxie.................... 

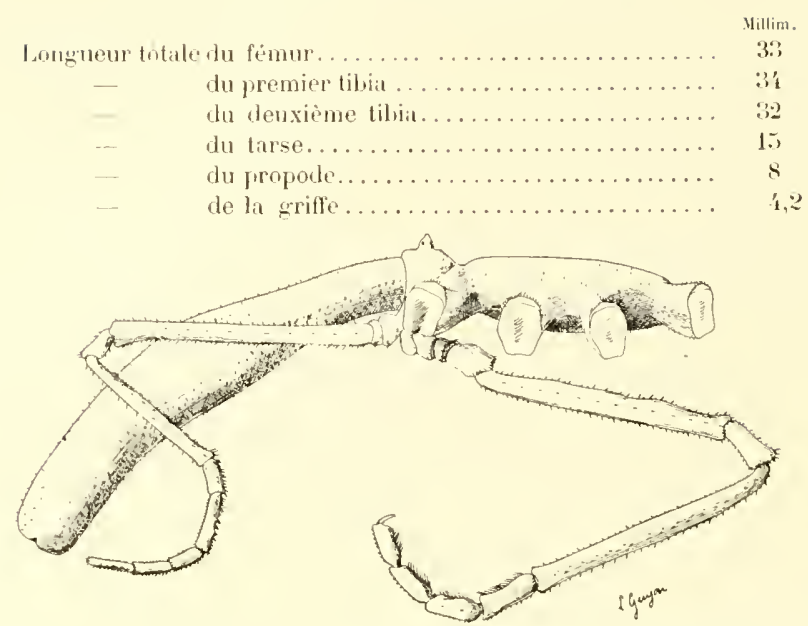

Fig. 20. - Colossendeis australis, ơ. - Côti zauche ave le palpe el lovigire. Gr. 2.

Patte rlroite de la troisième paire:

Millim.

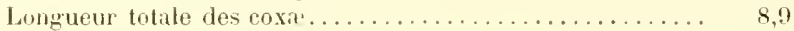

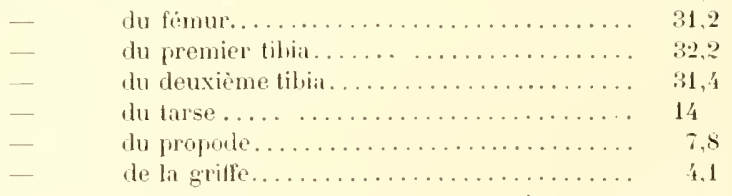

Cet excmplare ressemble tout i lail au type dereril par M. IIodgson; pourtant les doights des patles lic. 21 égalent en longuenr la moilie du

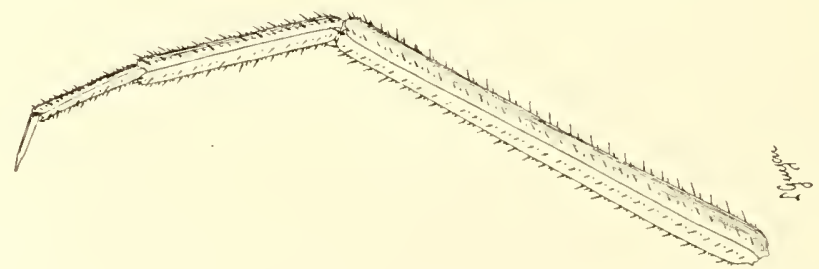

Fig. 21. - Colossendeis australis IIndgson. - Les 5 drmiers artirles de ba deuxieme palte druile. Gr. 2.

propole of so distinguent d'aillours par un rétrécissenent terminal fint bruspue, non signalé dans les types. Malgré un eximen tris mimutieux,

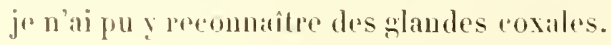


Distributux. - Les exemplaires types de la "Discovery ", un mâle et une femelle, furent capturós clans la provine alrioaine, l'un à l'ile Coulman, au large du cap Wadworth, par 8 a lö hrasses de profondeur, l'autre, an large des monts Erehus et Treror. par 500 brasses. L'esprere semble donc etre circumpolaire.

DEUXIEUE ORDRE. - TYHPHOWHORTH.1 h. I. Porock (emend.).

Premiere Famille. - NYMPHONID E P. I'. C. Hoek.

Des quatre familles que j’attribur à l'ordre des Nymphomonorphes, celle des Nymphonidés est reptaimement la plus primitior, rar lous les appendices y sont bien développres. Elle sr mattarle aux Pyonogonides ancestraux par l’intermédiaire te formes disparmes on qu'on ne comnait pas encore; le scape des chílicires, en effet, ne compte qu'un article, même dans le gente l'entanymphon Hodgsom, yui est dicapode, et tous les représentants de la famillr ont des palpes de cincp articles, a l'exception du genere P'aramymphom Caullery, qui en compte sept.

Outre ces deux gemes, la famille comprend les Vymphom Fabr., Thatomymphon et Boremymphon, lormes ortopodes tris voisines les unes des autres et qui dérivent vraisemblablement des /'entmmymphon pau" l'intermédiaire des Tymphon. On peut dire sans exisération que les Vymphom sont des I'mutamymphon octopodrs, los Chatomymphon des Vymphom trapus et pileux, à pinces courtes, les Boreonymphom des Chatomyrrphon aveugles dout les pinces sont inerues ot les epines des

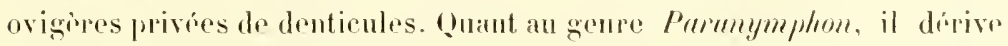
d'une forme plus primitive que l'esprece areturllement comme du genre

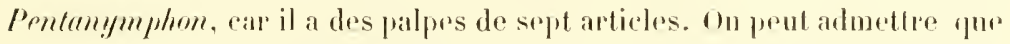
cette forme étaitun I'entam!mphrm à palpes richement articulés : la variation dans lo nombre des articles des palpes so nanileste dams lo grenre Decolopoda; chlo a pu tout aussi bien se produire chez les I'entrmymphom.

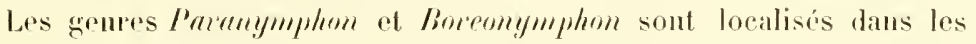
mers aretiques ou daus leur voisinage ot romptent charun mo espire; les trois antres genres ont des reprósentants daus lesmers antaretiqueset lournirent puelques rspieces aux naturalistes du " P'ourpuoi l'as?". 
Genre Pentanymphon Hodgson.

Le genre Pentamymphom a été fonde par M. Hodgson (1904, f.i9) pour une espiece curieuse trouvée par la " Discovery" dans la liaie Mac-llurdo. 11 prósente einq paires de pattes, mais est moins primitif que le genre Decoloynda, car le scape de seschéliceres est simple; ses palpes noont que cinq articles, et ses orifices sexuels sont moins nombreux, du moins rhez les mâlos. Il n'olfre aucune affunitó avec ce dernier grenre, et, en tout cas, ne saurait en dériver, ainsi que le pensait Il. Cole 1905). C"est un Nymphonomorphe tres typique et le plus primitif de l'ordre, au moins parmi les formes actuellement comnues.

L'unique espece du genre est le l'entanymphon antarrticmm découvert par la "National antaretic Expredition "; ce Pyenogonide présente des grilfes auxiliaires bien développées, et ses tarses sont à peu près de même longueur que: le propode.

\section{Pentanymphon antarcticum T. V. Hodgson.}

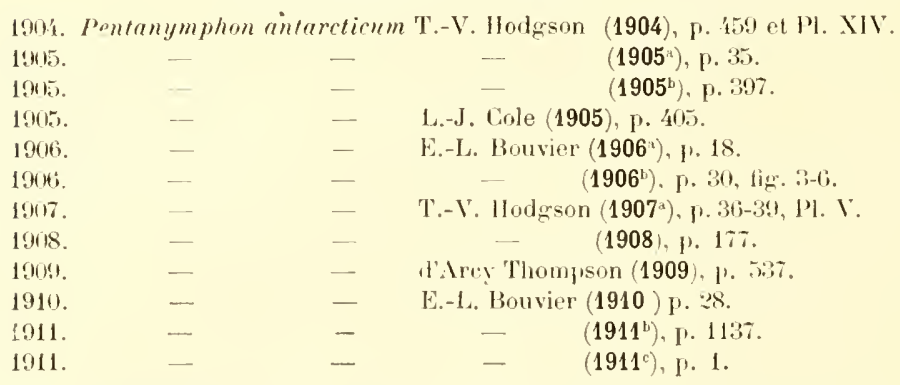

Les specimus recueillis par le "P'ourquoi l'as? "ressemblent à ceux du "Français " el different du type de la "Discovery " par la forme du céphalun, dont le cou est plusétroit tig. 2.2) etla partie antérieure bien pus dilaté, le rapport des largeurs de ces deux parties étant de $2, ; 0$ a 2,8 , tandis yüil se réduit à 1, , if dans la figure du tỵe domépar M. Ilodẹson. Lestibias I de res formes sont d'ailleurs relativement plus courts, conparés aux tibias 2; le raport variv entre 1 , 12 et 1,33 , tandis que, dans les figures de M. Ilodgson, ce nombre séleve à 1,38 ; souvent aussi le propode 
est de mème longueurouplus long que le tarse, alors que, dims le type, il est notablement plus court.

On croirait, dés lors, se trouver en présence do deux formes géographiques de la même espèce: l'une angusticolle lig. 2.2), l'autre laticolle (lig. 23): la premiere appartenant ì la provinee magellanique, oì chle a été trouvéc par le "Pourquoi Pas?" et le "Franceis ", l'autre habitant les parties australes de la province de Kersuelen, où elle aćtó prise par la "Discovery". 1)'après quelques indications domées par II. Hodgson (1908, 177) sur l'exemplaire capturé au Oreades du Sud par l'expédition antaretique écossaise, il semble que col individu présente surtout des analogies avec la première forme, ce qui fortifierait l'hypothese.

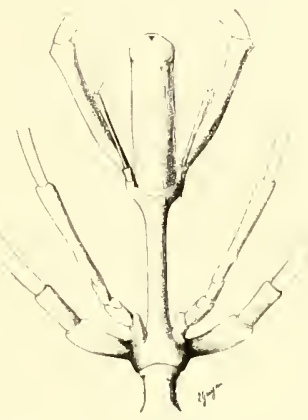

Fig. 22. - Pentanymphon antarcticum llorgson. - Trompe, réphaton a lase des opjendices correspondants. dans un individu or à long. con. ir. 8 .

Il ne parait guire prossible d'attribuer ces différences à la taille ni ì l'àge, encore que, dans lescullections du "Pourquoi Pas? ", le seul individu à peu près idenlique au type de $\mathrm{M}$. Hodgson soit plus petit que les autres, et presque de mème taille que l'exemplaire figuré par l'auteur anglais. Alstraction finte de cette coüncidance, les carac- Fig. 93. - Pentanymphon antarcticum. - partie anterieure du tères morphologiques corps dune of it cou bret: on voil, sur lat one coxa, le's oritires de l'espece nous apparaissent conme indépendants de la taille. H. IJodgson ne donne pas les dimensions maxima ef minima des exemplaires qu'il a etudiés, mais il olserve qu'elles " varient considérablement" sans entrainer de modilications importantes dans les raractères spéciliques. Et, d'autre 
part, il suflit dejotor un couje d'reil sur lo lableau relevé plus loin pour volr gu'un expmplaire peut être franchement angusticolle, avec une taille flus réduite que celle du type laticolle tiguré par M. Hodgson.

Ainsi le lentunymphom anturcticmm semhle bien représentí par deux formes, l'une angusticolle, l'autre laticolle, ayant chacune dans l'Antaretide une distribution propre. Il va sans dire que cette conception réclame le bénélice derontroles ultérieurs.

D'apres II. Ilodgson 1907,38 , les orifices sexuels de la femelle se trouvent sur la seconde coxa de toutes les pattes et sont assez distincts chez l'adulte. Les orilicess du màle sont toujours "difficiles à observer ", et l'auteur n’a pu les "aperceroir que sur les pattes des trois paires postérieures".

En ce qui concerne les récoltes du "Pourquoi Pas? ", je considere comme lemelles tous les exmplaires ou les orifices génitaux sont hien distincts; ces orifices apparaissent ventralement sur la seconde coxa, trís peu éloignés de son bord distal. Les individus ainsi laits sont un peu plus massil's que les autres et présentent des pattes un jeu plus fortes, surtout dans la région fémorale. Par ce double caractère, ils correspondent bien anx femelles de M. Itodgson, mais ce deruier auteur n'a pas indiqué la position des orifices génitaux dans les exemplaires qu’il a étudirs.

Par contre, je tiens pour màtes tous les exemplaires ou je n'ai pu déconvir les arifices sexuels. Ces exemplaires ont les pattes grêles; j'y ai dícouvert dorsalement, à la naissance du tiers distal de la deuxieme coxa, sur toutes les pattes, une légrire saillie oir s'ourrent sans doute des glandes coxales. Peut-ètre ces glandes coxales, comme les glandes sexuelles du màle, ne s'ouvrent-clles pas qu'à l'ápoque de la reproduction.

Dragage $1^{0} 111$ : 26 décrmbre I!Mos; chemal de Roosen, au nord de

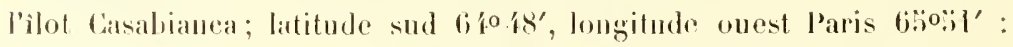

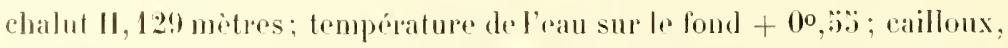
roche, vase. Trois exemplaires (No 22) "rouge-brique et blane ", dont les orilices sexuels u'ont pas été apercus. Ces trois exemplaires me paraissent itre desmalles; leur saillie porifere coxale est trèsnette, mais les pores y sont rarement visibles. Deux de ces exemplaires sont ì peu près 
de meme taille ot plus grands que le type liguré par Hodgson; l'atute esl plus pelit. Tous sont franchement angusticolles, mais l'un des promiers a le propode tris legienement plus court que le liarse. On trouvera les dimensions de cet exemplaire et du petit dans lo tablean do la page suivante.

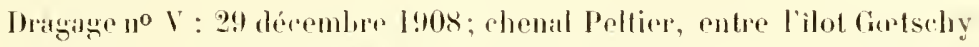
et l’ile Dommer; chalut II, 92 mobes; lemperature de l'eau sur lo fond

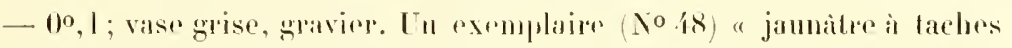
plus fonrés n. Cot exemplaire est mu angustirolle tros net à cou allongé;

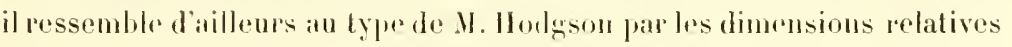
des divers articles des pattes. Il appatient probablement au sexo male.

Un second exrmplaim (1o 4!) ctait "jamne tres pàle" ; il ressemble tout à fait au précédent par sa taille et par ses pattes, où, pourtant, certains propodes sont anssi longs que les tarses.

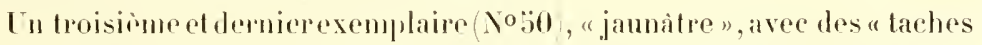
plus loneres tirant sur fr pouge ". Cot remplaire est également angusti-

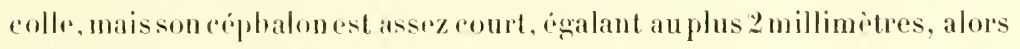
yur le cejhalothoras uesuro:; $\mathrm{mm}, 3$; les pattes et le trone sont plus massifs que dans les exemplaires précédents, de sorte que l'animal ressemble a une femelle; pourtant je n’y ai pu voir les orilices génitaux. Les propodes sont anssi longs ou plus longs que les tarsos.

Iragage No VII: I6 janvier l\$09, prisele la Terer Alexandro-Jer; atitude

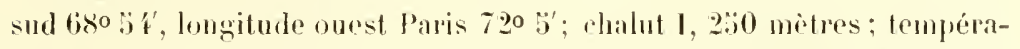
ture de leau sur lo fond $+10,6$; roche. Ln exemplaire (No 1:3!) " jamme pâlè .

Cet exemplaire (fig. 22) est un male angusticolle a tros longe cou, le rephalon mesurant 3 millimitres alors quelo cephalothorax atfeint $6 \mathrm{~mm}, \mathrm{~h}$; le frone ef les pattes sont d'ailleurs fort wheles, de sorte quo lindividu ast tres probablement un mile. Les pattes ressemblent tout it fait is collos du type figuré par M. Hodgson, le rapport do $t^{1}$ a $t^{2}$ atant de 1,37 (Voir le tableau de la page suivante).

Th secont exemplaire mile (No 160 , de mene coulenr, prosente les mimes cametères, abstraction faito du cou, qui est moins lumg̨ et phus épais, un peu moins pourtant que dans le type. 


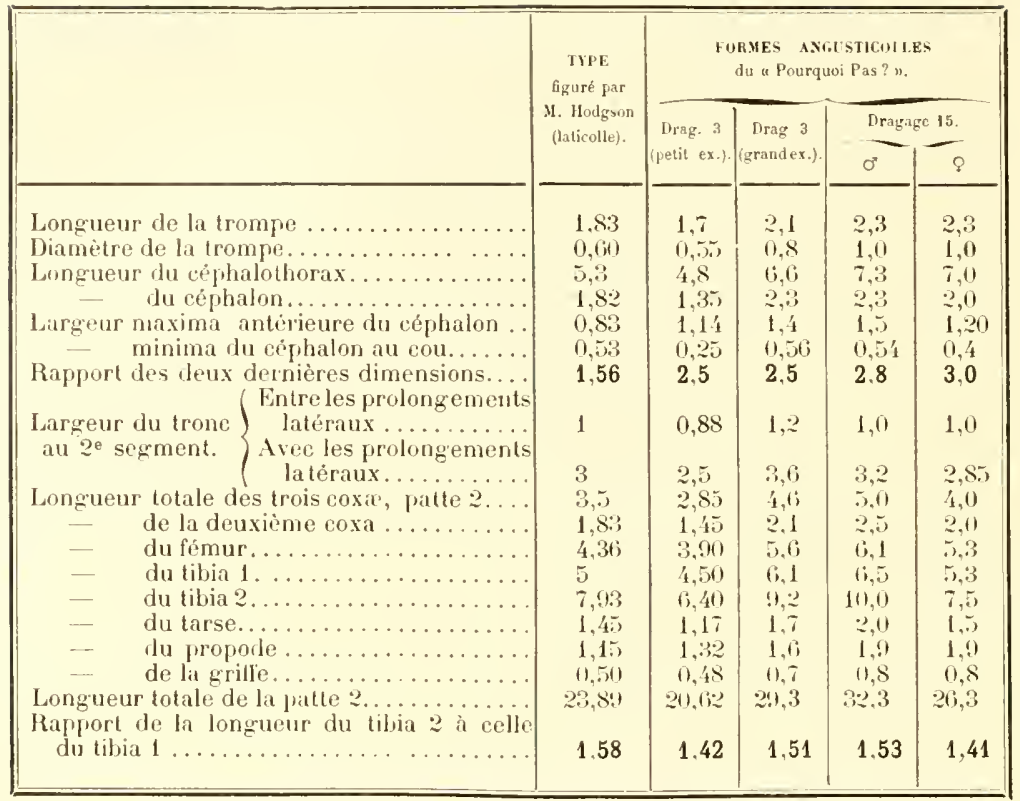

Dragage no XV : 26 novembre 1909, devant Port-Lockioy, chenal de

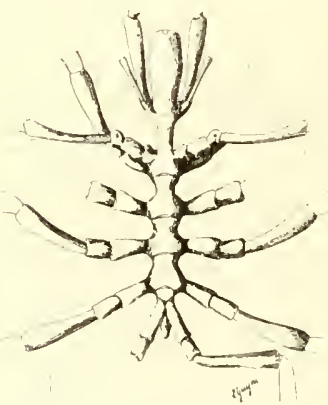

Fig. wi. - Pentanympleon antureticum, o* - Exemplaire à cou bref. O $14 n^{\circ} 651$. (ir. 4. coxa. Les quatre spérimens sont assez angustienlles, aver un con de longueur midioce lign. 23). Les dimensions du plus grand, que je tiens
Roosen; latitude sud 640 19', longilude ourst Paris 6.30 49'; rhalut I, 70 mietres; vase el cailloux. Quatre exemplaires de "coloration lrun jamnâtre clair arec, sur les pattes, des raies orangeres transversales et cireulailes ". Les exemplaires (No 6:3l) sont de srande laille, leur cíplatolhoras mesurant de 7 à 8 millimetres; trois sont des femelles oì les pores génitaux appraraissenl fort nettement; Ir quatrieme est un màle lig. 2i) où je nai pu découvrir d'autres pores yue reux silués sur la lace dorsale de la deuxiome 
pour male (tic. 2) i), sont relevers daus la yuatriome colomne du tableau de la page précédente.

Un autre exemplaire No (i.3.3), "jaune sale pàle ", ast beancoup plus petit, son éphalothorax ne mesurant pas plus dre 1 millimètres de lonģueur. Ciet exemplaire se distingue par la brieveté relative du céplaton, qui est angusticolle, le rapport de sa dilatation antérienpe da partie la plus étroite du cou ćlant de 2,6. Coest probablement un mâle.

Je relive, dans l. Lablean dr la page 70, les dimensions du type laticolle

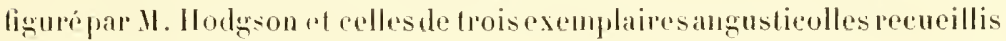
par le "Pourpuoi Pas?":

Genre Nymphon Fabr.

Les lymplom sont artuellement peprésentés par is especes environ, y eompris les quatre nouvolles recueillies par le "l'ouryuoil'as?" au cours de sa camprane. Cos espéces mo paraissent devoir ètre réparties en deux groupes: celles on les grifl's auxiliaires sont rudimentaires on nulles et colles on ces griflos sont bien développées.

Le second groupre est de beaucoup le plus vaste, ear il romprend toutes les espèes, a l'exception de 11 qui seront sigualées plus loin. Il se trouvedans toutes les mers, of celles situés au nord de l'épuateur n'en renferment pas dautres. La scule espece yui, dans ectte dorniòn region, pourait etre rapproches du promior groupe est le $Y$. longimammm G. O. Sars, qui habile la mor de Kara; tans cette lorme, en ellet, les gritles auxiliaires sont tris riduites et rgales au plus, en longueur, au dixiène de la srille principale.

Les especes du premier aroupe se trouvent toutes daus la region australe of presefur toutes dans les eaux subantaretiques ou antaretiques. Les semles qui nappartiennent pas a ces deux depniopes rigions se rencontrent an voisinage : le 1 . refpene llodgson dans les mers du Cap, he V. promerem, IIoek an large de Valparaiso el le. V. longicollume Hoek dans les eaux chilienmes. Les gribes auxiliares sont extremement réduites dans cotte derniene espere; clles font absolumont defaul daus les deux autres.

Jajoute que, parmi los s especes subantaretipues du premier groupe, une seule présente encore des grifles auxiliaires, qui sont d'ailleurs i 
peine pereptilules, cest le. s. stylops, une espèce nouvelle recueillie en abondance par le "Pourquoi Pas?".

Ainsi, alors que l'hémisphìre austral renferme des espèces des deux groupes, l'lémisphiore boréal n'en comprend aucune sans griffes auxiliaires; d'où it fiut conclure que les. Vymphon ont évolué plus vite, à cé point de vue du moins, dans les régions australes.

Les $V_{1}$ mmphm se timnent à toutes les profondeurs et se rencontrent fróquemmént près du littoral, même dans les paỵs chauds, où ils semblent d'ailleurs plus rares que dans les mers tempérées et froides. Leursespéces arctipurs ou subantaretiques sont au nombre de 22 et celles des rígions australes au nombre de 19. Ces dernières se distribuent dans les deux grompes et peurent ètre caractérisces de la maniere suivante:

Prenier groupe (grilfes auxiliaires rudimentaires ou nulles).

I. - Le tibia 1 niest pas sensiblement plus long ou est plus court que le tibia 2.

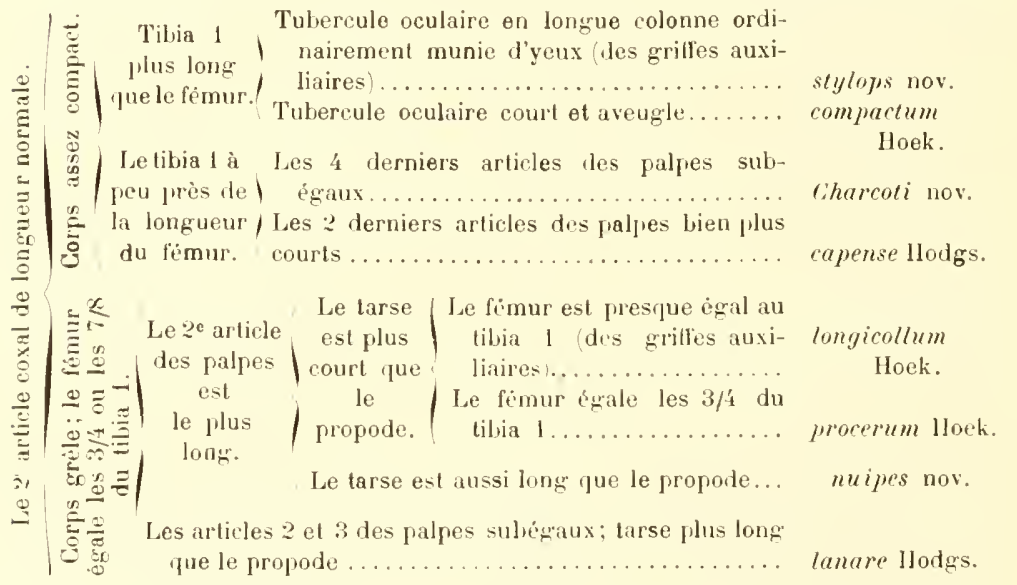

Le 20 article coxal égale environ les $2 / 3$ du fẻmur, qui égale la moitié

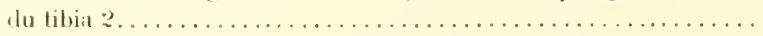

11. - Le tibia I plus long que le tibiu z, lequel est à peu près égal au fëmur.

rinces fortes à longs doigls, 2e article coxal normal, le tarse plus

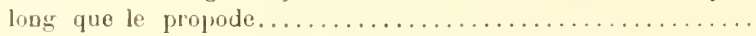
l'inces méliocres ì loigts médiocres; 2e article roxal très piriforme chez la $q$; tarse plus court que le propode........... proceroides nov. 
Decxime groupe (grilles anxiliaires bien développes

1. - Tarse ì pen près aussi long on plus long que le proporte.

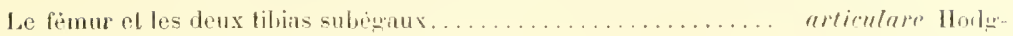

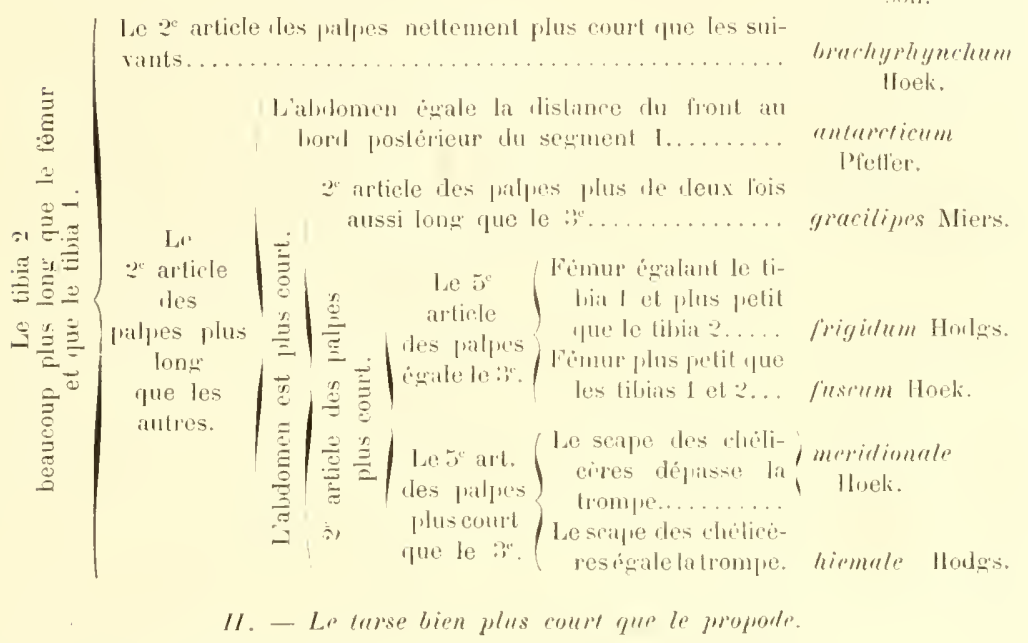

Tarse plus long que la moilie du propode; article? al 3 des pralpes

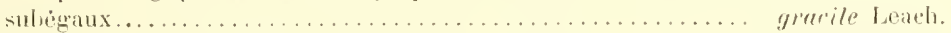

Tarse plus court que la moitie lu propode.

Articles? 3, a des palpes subejaux......... tridentutum

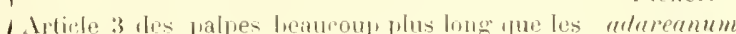
autres... . . . . . . . . . . . . . . . . . . . . Ilodgson.

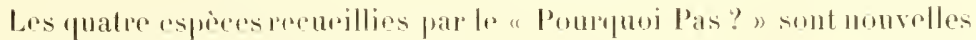
ot appartionment an premicus grouper.

Nymphon stylops E.-L. Bouvier.

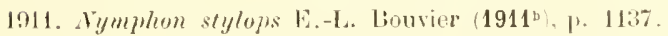

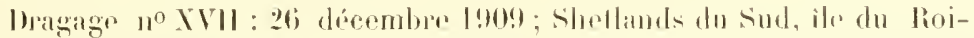

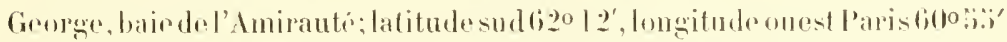

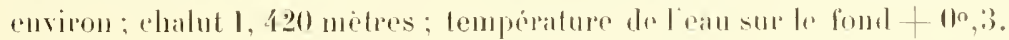

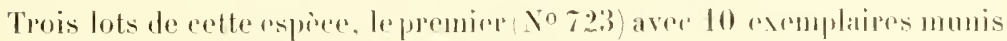

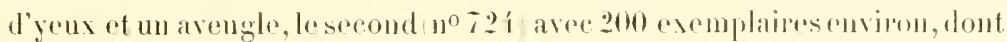

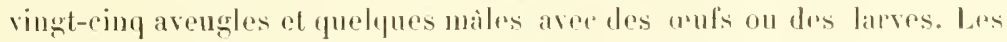
variations de ces exemplaires sont rifudieresplus loin (1. 7!); les màles à pru 
près en même nombreque les temelles. Dapris 11 . Gain, ces Pycnogonides sont "brun-marron", elcela se voit encore trèsbien dansl'alenol, où d'ailleurs la colorationn'es pas toujours muiforme, prineipalement sur lespattes. Les exemplaires aveughtes sonl presque lons incolores, dr même qu’un tres petil mombre diexpmplaires orules. Le troisieme lot (No 733 in aromprent que deus spécimens, qui sont areugleset incolores: sur tr vivant, d'après 11. Gain, ils étaient d'un "blane salde".

Vobri les dimensions de deux exemplaires très normanx, mn male, une femelle.

longueur de la trompe.

bianetre maximum de la trom

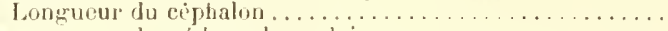

du pédoncule orutaire.................

Larmeur du cephalon dins sa partie anterieure la plus

larere. . . . . . . . . . . . . . . . . . . .

Largeur du ciphalon au cou. ..................

Longueur totalo du cephalothorax ................

Lar Ze segment.

Larmeur du céfhabothorax avec les probongenents coxaux

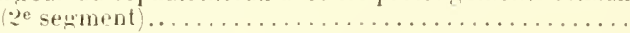

hongueur des coxit de la patle $2 \ldots \ldots \ldots \ldots \ldots \ldots \ldots$

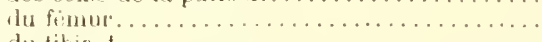

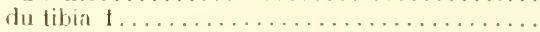

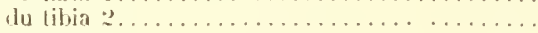

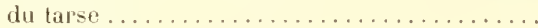

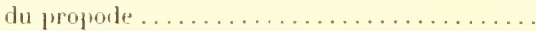

do la urilfe.

$\ldots \ldots \ldots \ldots \ldots \ldots \ldots \ldots \ldots \ldots \ldots$

\begin{tabular}{|c|c|}
\hline$\sigma^{*}$ & ક \\
\hline Mullim. & Myllim. \\
\hline 2,8 & $?, 2$ \\
\hline 1,25 & 1 \\
\hline 1,8 & $1, \pi$ \\
\hline 1,1 & \\
\hline $1, ?$ & 1,7 \\
\hline 11,85 & $0, \pi 5$ \\
\hline 5 & 4 \\
\hline 1,2 & 1,1 \\
\hline$: 3,7$ & $\therefore$ \\
\hline$\because 3,4$ & 3,5 \\
\hline$: 3, \because 1$ & 3.7 \\
\hline 5,8 & 5 \\
\hline 5 & 't, í \\
\hline $2: 2$ & $\because \ldots$ \\
\hline 1,8 & $1, \because$ \\
\hline$(1, !)$ & 11,7 \\
\hline
\end{tabular}

L. corps (tig. 20i ast comel el fort dans tontes ses parties, non sans

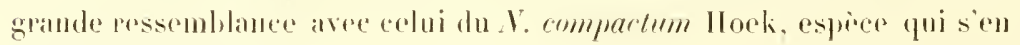
rapprochr le plus.

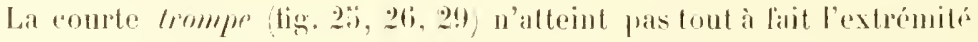
distale An scape des rhélieceres; elleatleint son maximum de largeur un

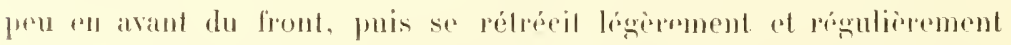
juspu’à la parlie antérieure; sil section transversale est mu triangle a faees un pro convexes et angles largenent amondis. Ceto forme se retrouve à son boul antérieur, an centre dutucl se trouve une depres-

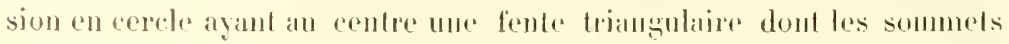


correspondent anx milienx des laces de la trompe; le sommot inlliórieur est silné sur la ligne módiane, les deux autres plus launt, l'un à droite, l'autre à gauche, sur le mème plau horizontal. La trompe est nue et ì peu pris deus fois aussi longue que large.

Le céphluelon (fig. 2:3, 26, 29) est court, en avant aussi large que sal longuemr, fort rétréci an nivean du con, qui est mu simple étranglement à partir lumpur se dilitent les parties antérieure et postéricure du crpphalon. Sur la partie postérinure séleve vertiealement lo tuberenle oculaire, qui deviont iei une colomm subeylindrivue presque

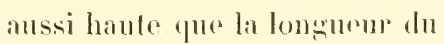

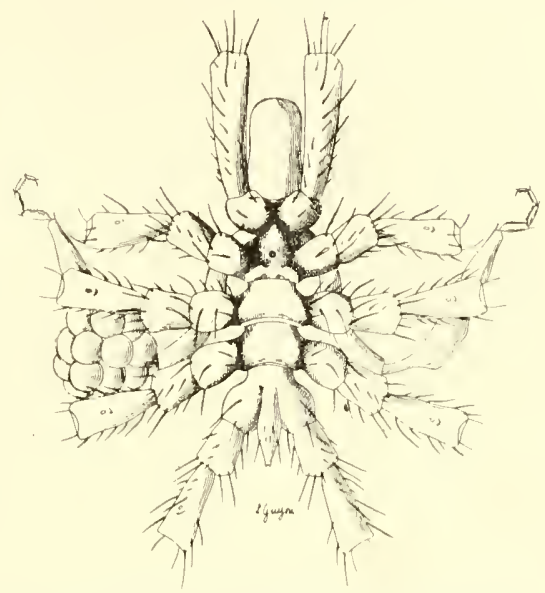

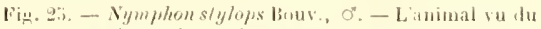

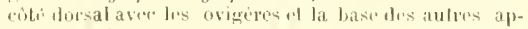

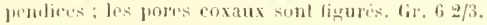

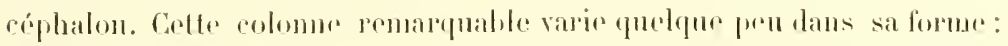
lantot elle est isodiannitrique depuis son épalement basilaire; tantòt elle se restricit un pen jusquan sommet : parfois, an contraire, elle se dilater légùmenent. Sia partie terminale, tres obtuse, est courerte pas les quatre yeux noirs, (jui sont presque toujours

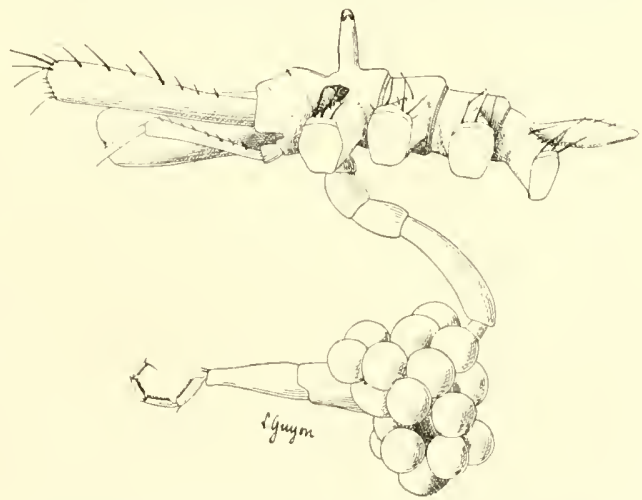

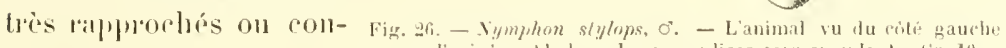
tigus.

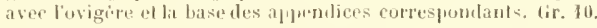

Le trone est épais el massil': il se emmpose de segments courts, plus larges que longs et nettement anticulés. Les probongements latéramx sout 
courls, ì prou pris de la lonsuem du druxieme segment et un peu dilités a partir de leme base; la distance qui les sépare a leur origine est atl plus égite au tiers de lenr propre largenr. Les souls ormements de ces parties du colpes sout de longues soies raides et dressées, situres sur les pro-

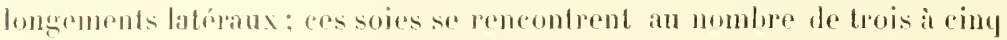
on six sur chaque prolongement latéral, afcompagnées dordinaire de quedpues soims beanconp plus comtes.

Lifludomen est horizontal, sans articulation hasilaire et nellement

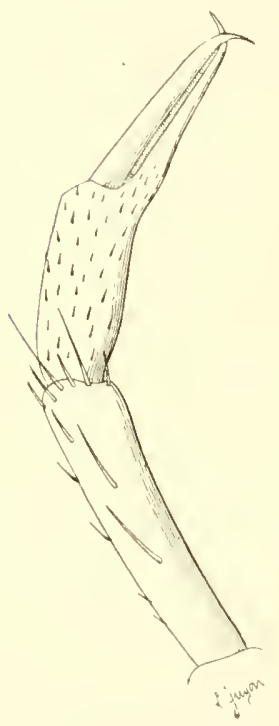

Fig. פ- - Nympleon stylops.

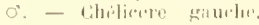
Gir. 16 . eomprimé diuss le sens dorso-ventral ; il ryale a pru pris on longuenr la moitió du tronce et, va du coté dorsal, prisente la forme d'une large lancette it sommet olutus. On y trouve un petit nombere de suies menthes ef fris courlus.

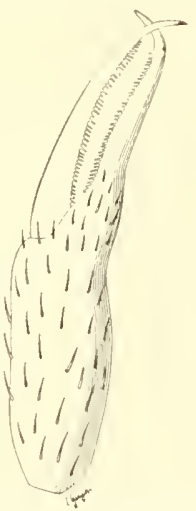

Fig. 28. - Nigmpleon stylops, o. - La pince de la rhihcirt gauche. fir. 28 .
Lescapedes rhelicipres (fig. 27,28 se dilate un pen de la hase atl sommet; il est dol'salrment orne de qualques soies longues of railes, insiries, avordes soies róduites, sur des saillies transverses peu maryuées; il y a nolammont un gromper de trois on quatre graudes soies pris du bord antérieur. Les pinces (fig. 2s) ne sont pas semsiblement plus longues que le scape. La proption palmaire

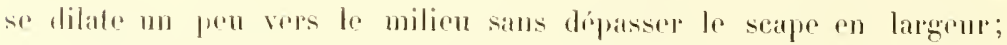
elle n'est pas da lout comprime of propte quelepes breves soies. Las doigts sont i preu pres anssi longs que la portion palmaire, un peeu

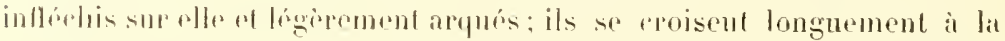
pointe ef portent sur feur bord intrine une armature continue de dents

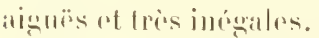

Les pulper altriguent ou dépassent it peine lat base des doigts des ché- 


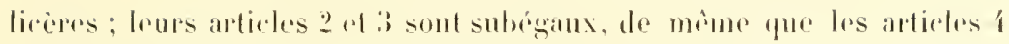

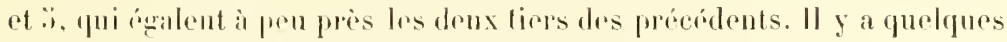
soies raides sur les diver's articles des palpes.

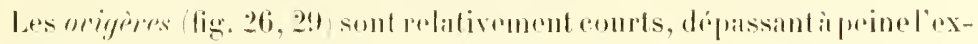
bémité distalc deslémursantérienrs. Lent troisieme article est un peu plus longre que les deux prónedents, a les quild depniers sont presque subégaux, encore que progressivement un peu phus courts de larticle 7 an loe; colni-ei so termine par moe grille línue, mais pour le moins anssi longue que las deux tiers de l'article lui-mòme el armóe de sept a huit denticules spiniformessur son bord interne. Les

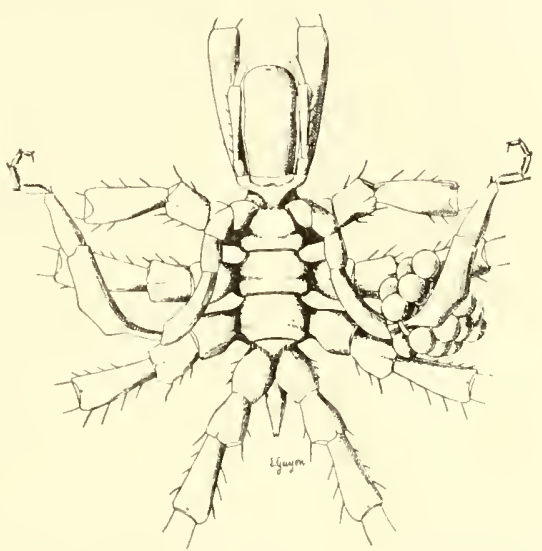

Fig. 29!. - Vymphon stylops Bous., O'. - Lanimal vu du roli: vintral, abec les ovigeres, lit base des autres appendices ef les proris seruels. tir. to $2 / 3$. Epines barloclées des artieles

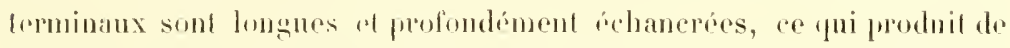

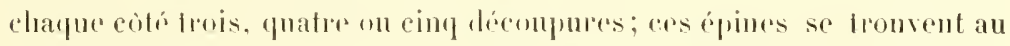
nombre de neul'à onze sur l'artirle 7 , de six on sept sur les deux suivants, of de sepl ou huit sur l'article 10. Il y a de nomberses soies sur ces articles, sumbut is lemp bord distal, oi elles devirmment plus longues. Les mâles se distinguent fortement des lemelles par la structure des articles

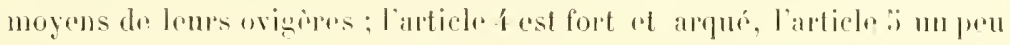

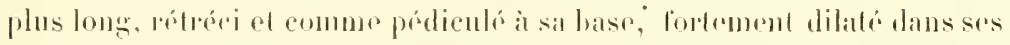

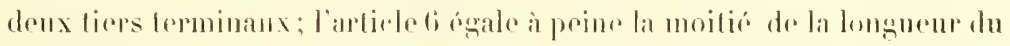
prédent et, lisse comme lui, également lépurver de puils, se dilate

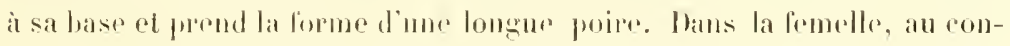
traire, res articles sent tout ì lait mermanx; droits, munis de queldques poils et sans dilatalion ane une, larticle fi bant il peine plus court que les deux autres, qui sont suberanx.

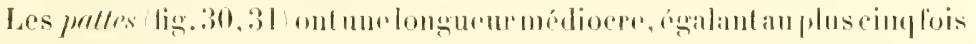


la longuenr du céphalothorax ; elles sont plutot fortes ef épaisses, surtout dans la rígion fómorale. Le second article roxal se dilate régulierement de la bise au sommet of égale à peine on longueur les deux autres

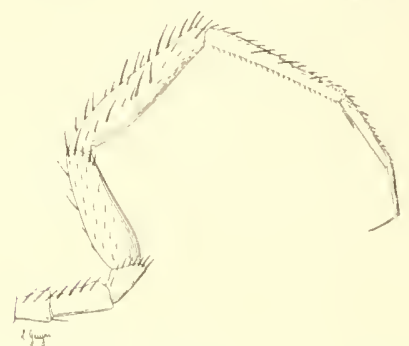

Figr. 30. - Nymphon stylops. 0". - La go palle
Iroite. lir. 5. articles coxamx rúmis. Le fémur igite en longurur les trois quarts environ du tibia 1 , lequel rigale en longueur le tibia 2, on le dépasse

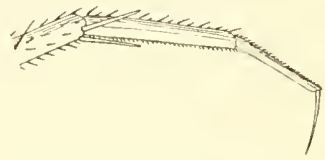

Fig. 81. - Nympteon stylons. O'. - Extremité de la Qe patte ilroite. Gr. 10.

Iuelyur peu, cela variant d'une patte ou d'un individu a l'antre. Le farse et le

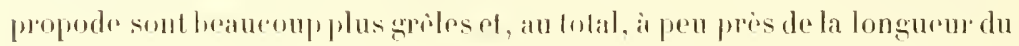
fómur ; le propode égale à pru pros les quatre cincuiomes de la longueur du tarse. La griffre, tris faible, igale au moins la moitic du propode; à sa base, dorsalement, sur le bord distal de re denier, on voit an microseope denx sprifles auxiliaires ayant environ un dixime de millimitre. Lat ligne latérale n’est pas distincte, sauf sur le tarse el, à un moindre degré, sur le tibia 2. Les lomges poils dressés des prolongements latéraux du tronc so retrourent sur les coxir un peu plus nombreux; ils se retrourent également, daillemes épars, sur les trois artieles suivants: les deux soirs de l'extrénite distale du tibia 2 sont partienlirement fortes ret presque spinilomes. Les deux articles suivmts ne presentent que has soires peu nombreuses ot fort réduites.

Les orifices sexuels tu mald tig. 299) sont extrimement petits et tris

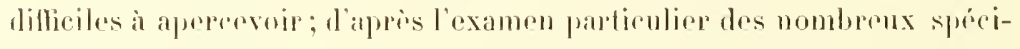
mens de la collection, je crois pouroir dire yn ils sont tocalisés sme los pattes des deure demieres juires, on ils se trouvent sme la ligne médiane ventrale do la deuxieme eoxa, à une tris pulite distanee du hord distal. Hène sur ars pattes, je n'ai pas toujours pu les apereevoir, of je mo

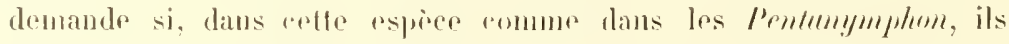
ne sont pas rolos it reptains moments. On a vo que les mailes se distin- 


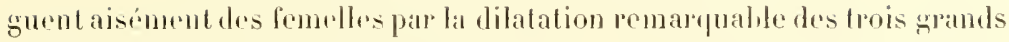
allicles moyens de leurs ovigines.

Les orifiees sexuels des frmelles somt assez srands, ovalaires ed plus

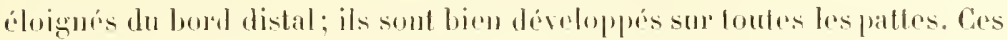

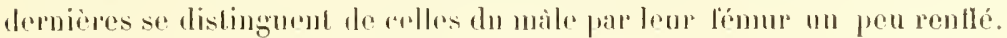

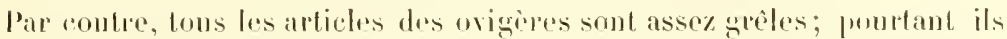

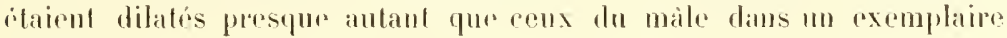
femelle.

Sur plusieurs centaines d'exemplaires de ertte espiece, je n'ai trouró que dix miles chamés de: la progeniture, huil avee dos mufs, les denx autres avee des larves. Les and's forment sur chaque ovigere un pelit

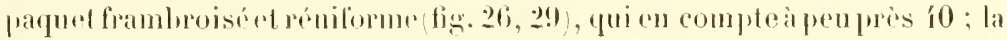
masse varie du bleu rougealre an jaume; olle se rallacho il l'article "i des

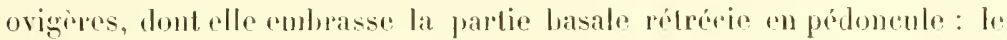
cément forme une sorte de eravate qui passe eomme un pout alo-elessus

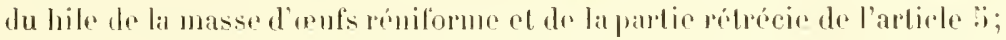
cela constitur un colliw sans ancune adherence ave l'article et qui peut

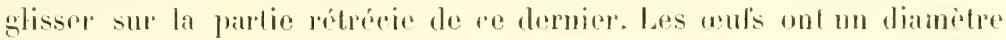
de 600 a i $700 \%$ \%

Les larres sont acerochées par lours pattes aux lambear athérents du sac cémentaire qui reliail leswul's. Je hes ai toujours lrouvérs hexipodes,

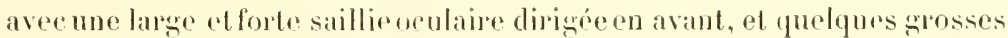
ipines au bout des anlirlesdes appondices. Ni palpes, ni ovigires. Le larse n'est pas rncore sciparé du propode; les grilles auxiliaires et les grilles principales somb relativement plus longues que chez l'adulfe.

Iarialinas. - Ie foutes les varialions de celle espexe, les plus imporLanlos de beaucoup sonl celles pelalives aus yeux. Noussavons dojà que les tubereules colummaires qui les portent sont variables daus leur forme, tantot phus rofrecis à la base qu'au sommod, tambol phus larges, le sommot lni-msume, ordinairement oblus, pouvant devenir aigu, ou former demx ou gualer sallirs. (Mant aux yeux eux-mèmes, ils sont normalemont au nombre de quatre, mais prósentent des varialions extraordinatres qui

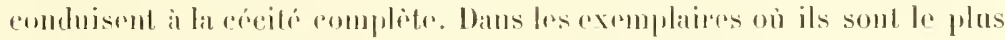
devoloppes, ils conlluent totalement nt ne prósentent pas de limiles 
distinctes, puis on les voit se séparer deux par deux, puis tous les quatre, et alopse réduire de plus en plus juspuà disparaitre. A rai dipe, les

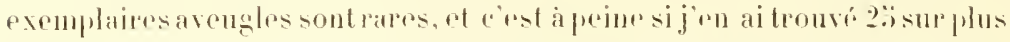

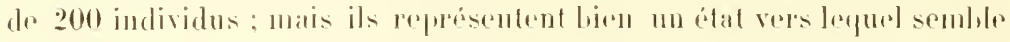

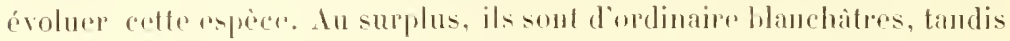
que les autres ont prespue toujours une teinte brunatre plus ou moins foncén: d'ordinairnansi. heurs soies sont plus longues et leur colome oeulaipe plus étreite. Hais il ne saurait etre question d'en faire une variétí ou une especer distincte.

A er sujet, il convient de signalor deux rxemplaires oi les pédoncules oevlaires sont extrabrlinairement anopman, en forme d'un haut ot large rone aigu, doul la base cmtrasse foute la largenr du céphalon : l'un de ces exemplaires a des yeux riduits; lautre est complitement avengle.

Il convinut de signalep également do variations assez notables dans la longueur relative des deux apticles tibiaux et dans lá position de l'abdomen qui s'incline assez liréquemment of se dirige alors un peu vers le haut.

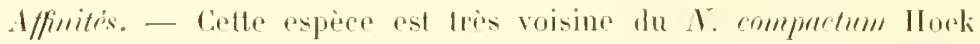
(1881, 11, P. II, lig. (j-8; Pl. XV, lig. 10), yui s'en distingue: fo par ses tubercules weulaires toujours avengles of d'aillmurs réduits à un simple bouton obtus bluml hmml): 2o par les doigts de ses chélicères beaucoup phus longs que la portion paluaire : 30 par la struchure des ovigères du nàle, oì, d’après la figme de X. IJodgson 1908, Pl. I, fig. :̈), l’article :̈ est peu rétréci à la base ef l'article fi dilaté sur jpestpue foulr sa longueur et presque aussi long yue le quatrieme: fo par l'absence degrifles anviliaires.

Le N. rompactum fut tronvé praple "Challengers ", par 1100 brasses de profondeur, à l'est d'Auckland, latitude sud 40028', longitude anest Grenvich $177^{\circ} 43^{\prime}$; daprès M. IIodgson 1908 , 168, l'expédition antare-

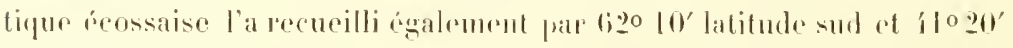

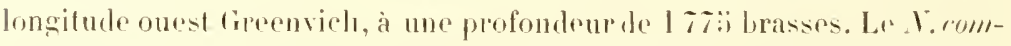

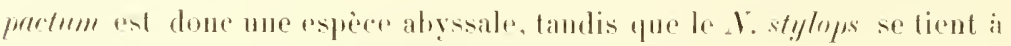
deplos faibles profondeurs 920 metros. 
Nymphon Charcoti E.-L. Pouvier.

Io11. Nymphon Charcoti E.-L. Bouvier 1911\%, [. 11:3.

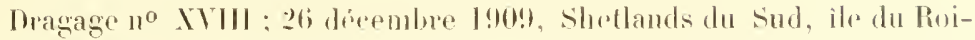
Georges, haie de l'Amiranté; latitude sud 6 zo I 2', longitude onest Paris

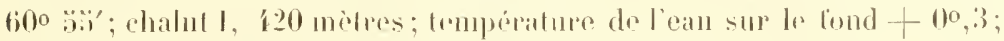
vase, eaillous. Trois exemplaies ( Yo Fig), dont dems màles of un

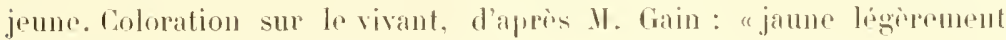
orangé n; la pigmentation a complielement disparu dans la alcool.

Ln exemplaire mutilé dr plus grande taille a déte clomé à M. Liomville No 730 par un pècheur norregien, quil'avaif pris sur une lialónoptère portant des Penella; localilé : Chili austral.

Les dimensions du male of de la femolle types sout lessuivantes:

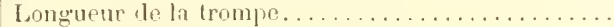

Diametre maximun fle la trompit. . . . . . . . . . .

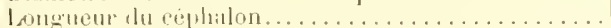

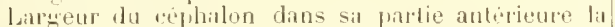

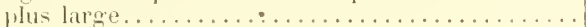
Largeus du céphalon au eou. . . . . . . . . . . .

Diamètre du tulsercule orulaire................

Longueur totale th cephabolhorix . . . . . . . . . . . .

Largeur lu exphabolhorax dans Ja jautie intérienre

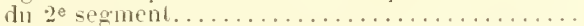

Largeur du whalothorax ave les jrolongrments coxaux ?e secment . . . . . . . . . . . . . . . .

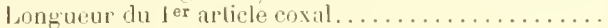

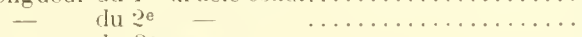

- dufinur.

du libia $1 \ldots \ldots \ldots \ldots \ldots \ldots \ldots \ldots \ldots \ldots \ldots \ldots$

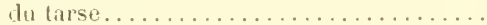

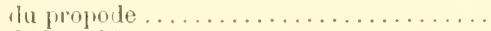

le In crille.
- this $3^{\mathrm{e}}$

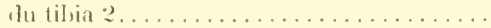

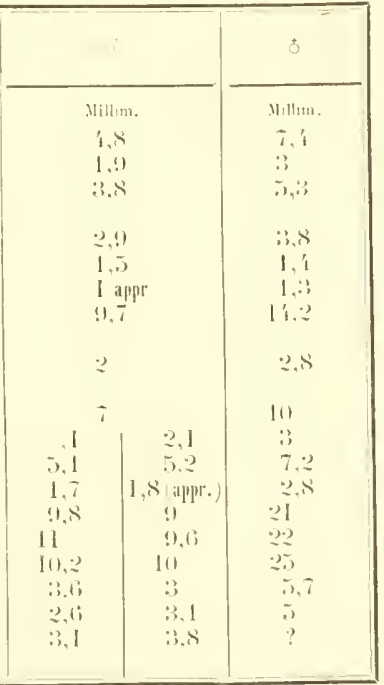

Dans le mile, lespattes mesmbers sont la druxieme et la qualriome du coté gaucho; dans la femelle, la fuatrienne du còté droit, la seule à peupres totalement intacte.

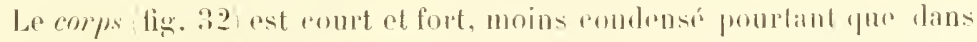
Ir. Y. stylops el le I. compurtume.

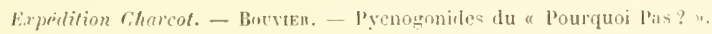


La trompr lig. 32 ressemble beatuoup, far ses dimensionset sa forme, ac celle du . I. stylops, mais elle est heaucoup phs nettement prismatique. encore que ses angles obtus soient remplacés par des faces arrondies; comme dans te Y. compuctum, sa partic dilatée est un pen rétrécie vers le

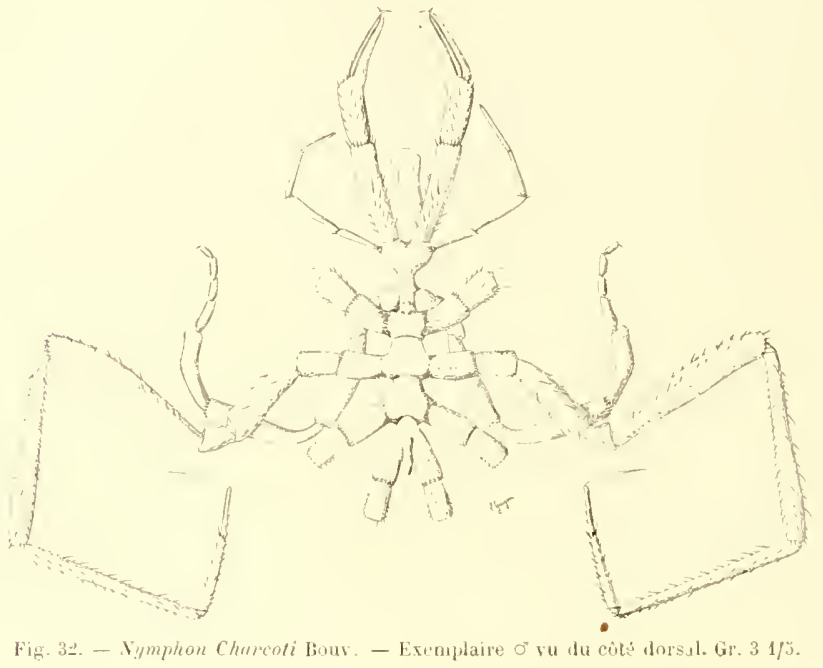

milieu. La fare luecalo de la trompe est un triangle dont les larges sommets sont olhusot legipement bilobés.

Le réphulon est fortement et presque ézalement ditaté en avant et en arriere, cesdenx dilatations étant rémies parmu con plus étroit demoitió; il ast phos large que long. La dilatation antérienre prísente un sillon midian tui s'atald of se perd entre les deux fortes sallies w prement base les rléliciores. Le tubereule oecupe la moitié postérieure

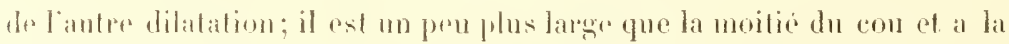
formor d'un faible dome lisse, rógulierement arpondi. Vers le sommet du dime se tromwent quate petits yeux triamgulaires largement séparés par un intervalle ro forme de frois. Mans lexemplaire femelle, le dome oreulairerest rétrici a la base.

Los segments do fronesont un peu dilatés rn arriere très nettement

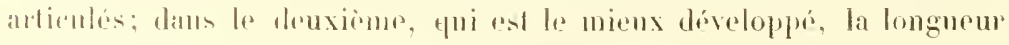


est notablement plus grando gue la bageur et un peu plus firible fue les prolongements lateraux, qui sonl prespur ylindriques. L'écartement des prolongements lateraux augmente de la base il la parie distala; il asl mo peu variable suivant les patles el, dans sa partic la plus large, il atloint au moins la largeur des prolongements hatiraux.

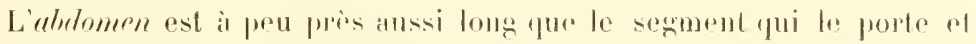

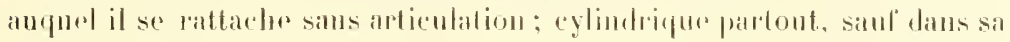

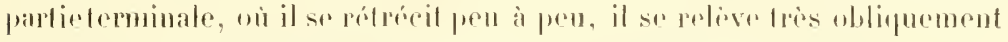
vers le hant, formant aves l'horizontale un angled dous moins boo.

Ontre les poils frescourts at tres tins, prestue impereptiblas, yui so

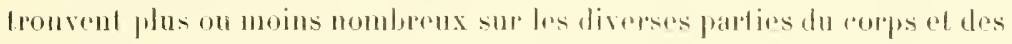
appendiees, le trone prosente dorsalement puetpues soies dressios fortes et assez lomgues.

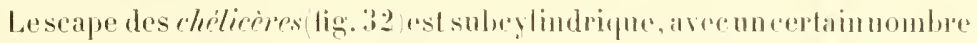

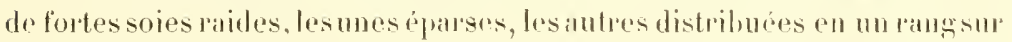
le bord distal. La pince est bien jitus longue gur fo scape et assez forte-

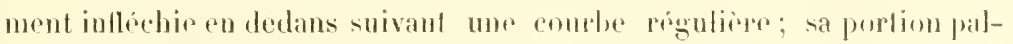
maire se dilate un peu de la hase an sommet et presente quolpurs soies dorsales; ello est nolablement plus courte que les doighls, qui se distin-

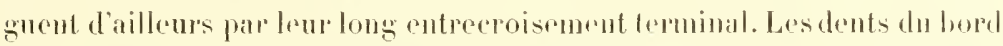
interne des doigts sontrepresentérs par descipines grèles, les unes longurs, les autres plus courtes, qui altopnent reguliemenent; il y a d'ordinaine

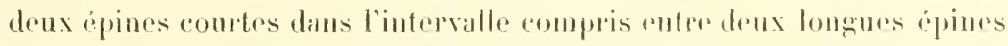

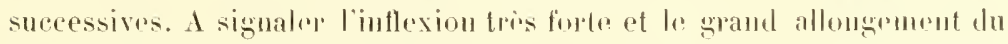
doight mobite dans sa partie teminale, qui prend ainsi la forme d'un erochet.

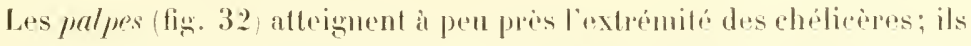
se distingment par les dimensions presque ryales dre lroms quatre derniors articles, surtont chez la fomello; dans le mile qui nous a servi de type, les

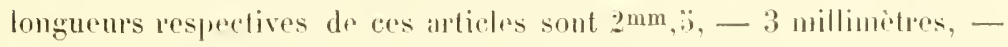

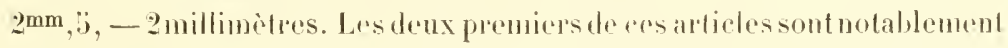
phus forts que les articles lemanaux, dont le dermier est particulière-

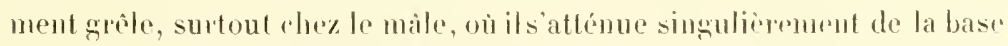
au summet. Il y a de petitrs soirs raides assez mombreuses sur les 


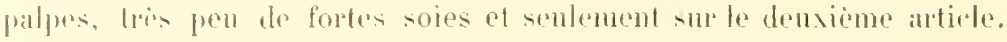

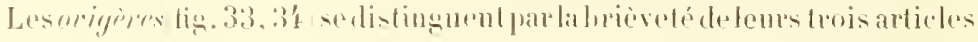

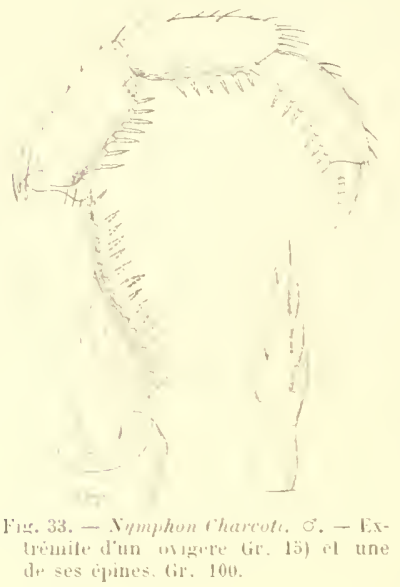
basilaress, surtont des deus premiers, par la grande dimension de l'anticle ti. qui est presque aussi long yue larticle $i$ el autaul fue les atlicles $i$ et 8 reunis, enlin par la longurur à peu près identique des articles

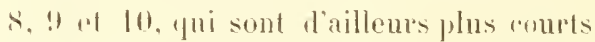
quelarticle 7 . An smplus les dillérences sevmelles ur sont gure moins accentues que dans le 1. stylopes: thez les mates lis. 3: l'article it mol large et presque droit : larticle $;$ lim plus long, dailleurs tordn, incurvé. fortement et ringulièrement rétréci il la base; larticle li a subi une torsion ens fort sensible et d'aillems ne se retréeit pas notablement de la base an sommel. Lans la femelle lig. 3í , ces trois articlessont deoits, se dilatent regzulierement de la base à la partir distale, ot si larticle f́ présente une légère in-

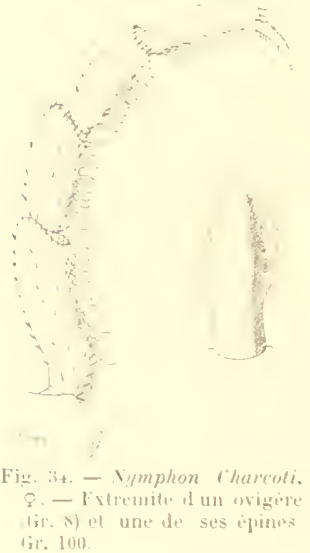
hexion, les antrus restent absolument droils. Les grilles sont mo peuplus comtes que larticle 10 dans les dens sexps, ave un bien plus grand nombre de dents spinilimes chez la femelle if que chez le màle $\because 3$. Les épines barbelées des ynatre derniers aribles sont analogues dans lor deux sexes, post-it-dire longues, grides, toutefois phus grêles chez le mâle fig. 33 et avec des larbelures latéralesplus fines ot moins nombrouses. Lo nombre de ces ipines presente des variations ponsiderahles: dans lovigere droil du mile type. ces rpines sont all nombre de 11.

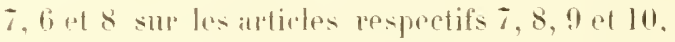

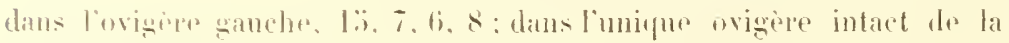
femelle (1), !. 9. 11. 


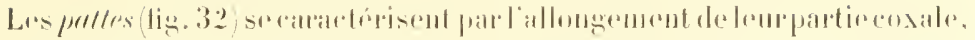

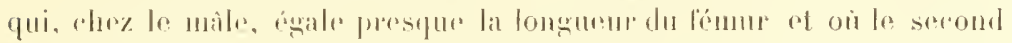

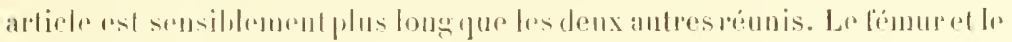

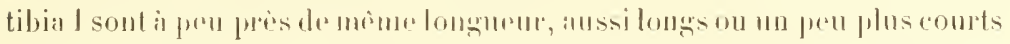

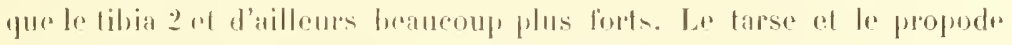

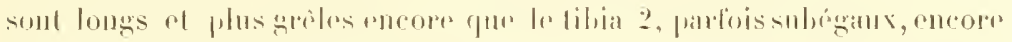

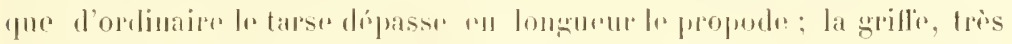

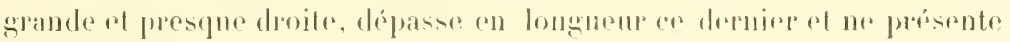

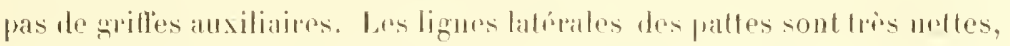

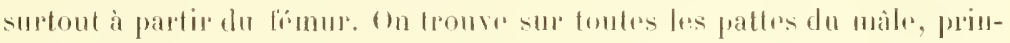

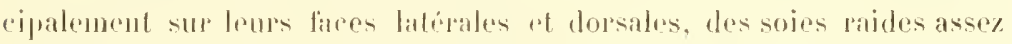
nombreuses at incegales, dont certaines sont phutrit longues. les soies

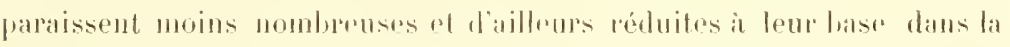

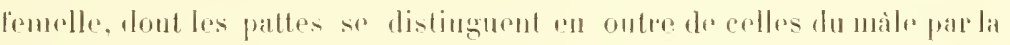

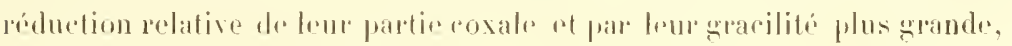
surtout dans la regigion frimomale.

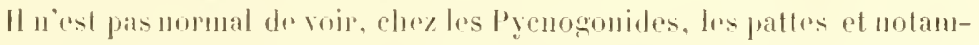

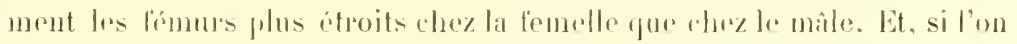
observe d'ailleurs yue noter femelle secarte du male par sos téguments

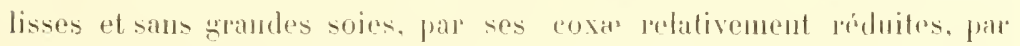

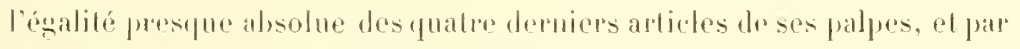
l'inégalitébeanconp moins suande dus fiurs dents spiniturmes deses chélicères, on est en droit de se dermander si cot exemplainr lemelle n'appartient pas à mo autre espece fur le male, à une espece dialleurs très voisine. Cela rest lien possible; mais il est trés possible anssi, el à mon sens phus probable, que les differences procilins sont dues anx dimensions

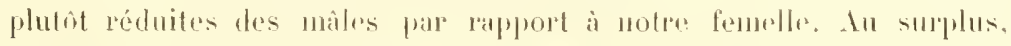
l'espice comptrecertainement parmi les Tymphom de grande taille, car le premier exemplaime du dragage $\mathrm{X}$ III, yuoiqur assez voluminemx deja son

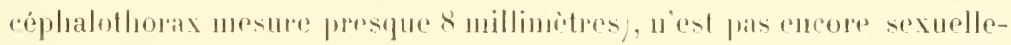
ment difliencie.

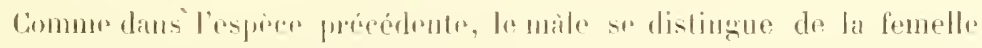

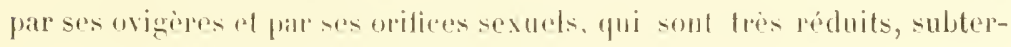
minamx et localisés sur les pattes des deux dermions paires. 
Alfinitris. - Cotte esprece est nettement calacterise par ses palpes, ses coxie, ses tris longues grilles, sa forte taille; on me siturait ha comparer a

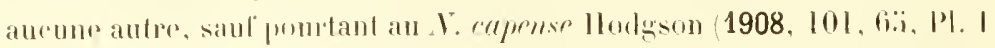

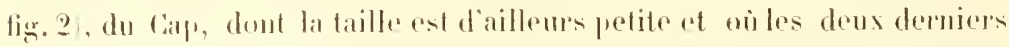
artiplesdes palpus sont hien plus comts que les drux précrédents; jajoute

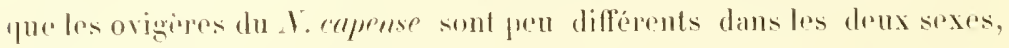
avee lo quatriome anticle plus long que hr suivant, alors quil est beaucoup plus court daus l. V. Thurenti.

Nymphon tenuipes E.-L. Benvier.

1911. Nymphon temuipes E.-L. Bouvier (1914'), P. IISi.

Dlakage $1^{\circ}$ XVII : 26 décembre 1909; Shotlands du Sul, ile du RoiGeorge, baie de l'Amirauté; latitude sud lizo 1 $\underline{2}^{\prime}$, longitude onest Paris

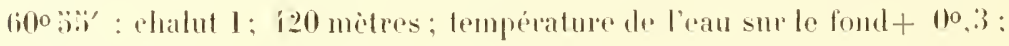
vase, caillunx. In cxemplaire immature, "l'un janne pàle sale ".

Les dimensions de cet exemplaire sont les snivantes:

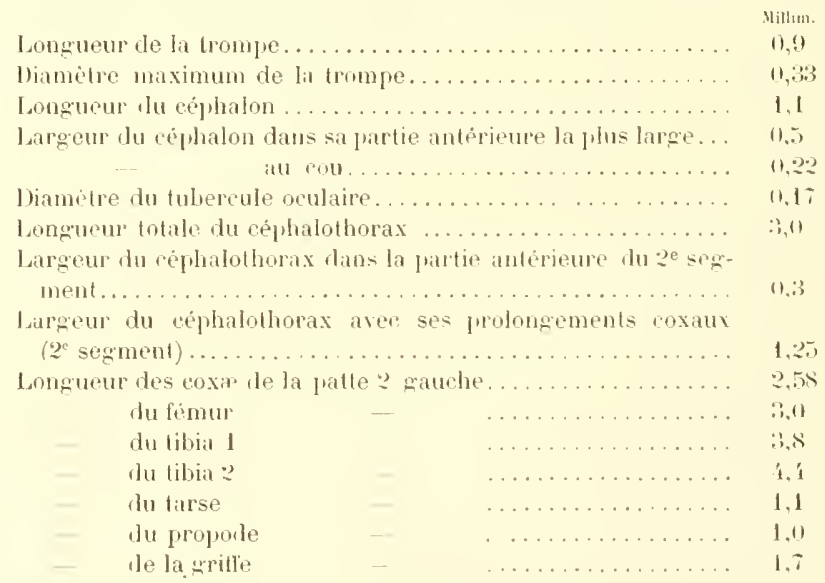

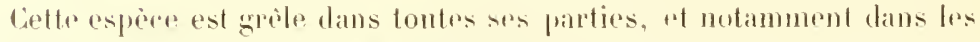
pattes, qui sont d'une temmité extrème. surtont à partir des tibias, d'ou le nom yue je lui attribue.

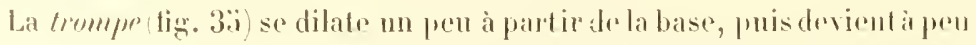


près subeylindrique; cllorst lorte, courte, dépassant à peine l'article 2 des palpes et n’atteignant pas, il s'en lant, l'oxtrémitr du scape des cháliceres.

Le cephalon lig. 3ij) est assez allongé, avec un rou plutót étroit et la partie antérieme fortement dilatée. Le tuberculo oroulaire forme une saillic larere assez élové, it sommot arrondi ; sur ce sommet on distingue denx bandes pigmentabes diun brun rougeatre lormant coix; mais re the sont pas là des yeux, car on ofiserve dos trainnes pigmentaires analowues en dautres parties du corps. siles yeux existent, ce qui me parait peu probable. ils nont pas, dans lo sprénen, unr enloration qui promelle de les distinguer.

Le frome rist rolativment émoit, aree des fmolongemunts légerement dilatés de la base au som-

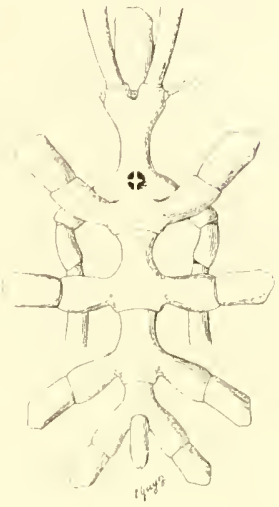

Fig. 3us. - Nymphon tenuipes louv. - Vue dorsale aver la lase des appirndices. Gr. $1+1 / 2$. met, of birn plus étroits que les intervalles qui les síparent. Commo les autres parties du corps, il est assez transparent, mi, et semble totalrment dépourvn de poils. Ses segments sont réunis par des articulations trés nottes.

L'abdomen, par contre, ne jursente pas d'articulation basilaire; il est à prine plus long que las probugenents coxam posterieurs ef presque verticalement polevé. Cylindrique ì la base, il s'alténue enconrau sommel.

Les chelicrees liz. 3ti sont linbles, mais assoy longues, avrequelques rapes soies dresserssurla fice supérirure de leur seape. Lapince est à peuprès de la lomguem de erdemier; ellen'ust pas sensible-

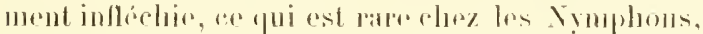
el se dilate régulierement drepuis la hase juspun"iu ni-

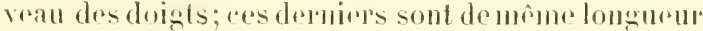

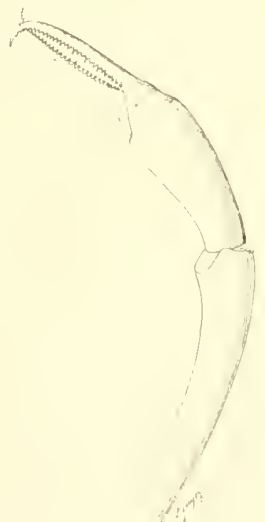

Fin. 36. - Nymphon lemuipes. - Chélicère. Gro, 3t. yue la portion palmaire ef reguliemenent recombes en are dans leur parlie distake; leurs bords internes sont armés de fines dents spiniformes. 
Les palpes ligg. 3i atteignent a pen pres l'extrémitr des pineses; leurs drux derniress articles sont subégitux; fo deuxieme est de beamroup le plus

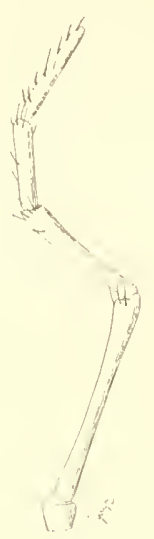

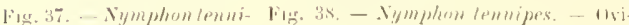
jos. - Palpe. Gis. 36.
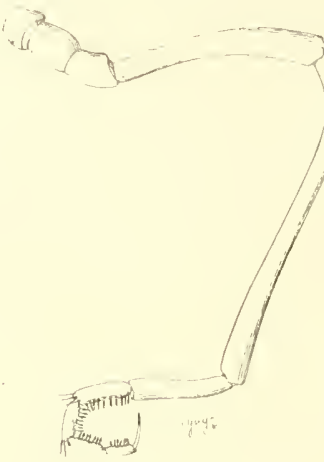

gere. Gr. 1 ; $1 / 2$. long. Longueur des quatresterniers articles: $0 \mathrm{~mm}, 72$.

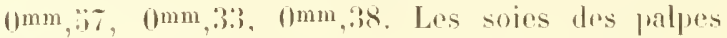

sont filles, assez nomlpenses, d'ailleurs peu allongres.

Les migieres (lig. 38) sont remaryuables par la torsion lígèrmentsigmö̈de el l'extrime allongement de liatlicle :i, qui rigale plus de trois fois la suivant et nue lois a demir le précédent. Co dermierest réguliepement arpuce. Les quatre derniers articles (fig. 39) sont comrts, subrigaus, encore qu'ils diminuent

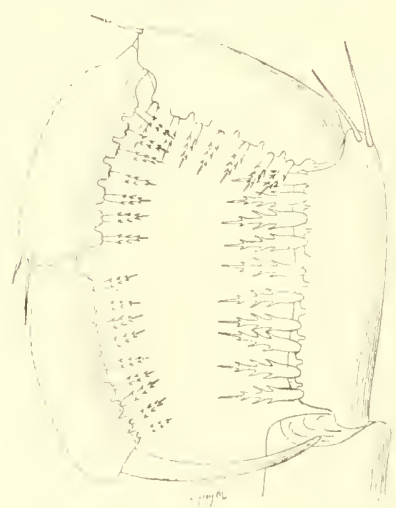
un pen de longueur du premier alu depnier; lents fiphes (lig. io) se découpent assez lomgnemenl sur les borts; jon ai

Fin. 39. - Dymplon temaipes. -

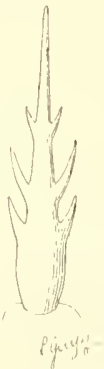

Pis. 20. - Nymphom tenuipos. Lue ipine des ovigeres. tir. 3xi. complí dix i douze sur l'artiche La gifir est allssi lomgue que le dermiers arliclr. Les ovigipers sont grejles sur toule leur stemdar, arre de courtessoirśpiparses.

Comme ou lo voit par lu tableau de la page s6, le fémur(tig. il est un pen plus lomge que los coxar, nolablement plus court que lo tibia I of 
beaucoup plus que le tibia 2; le deuxieme arlicle eoxal s'blumit rónulièrement de la base an sommet el dópasso en longueur los deux articles conligus rémis. Le lane est in peine plus long que le propode et bien plus court que la żrille, laquelle est légècementarquéce, sans rudiment apparent

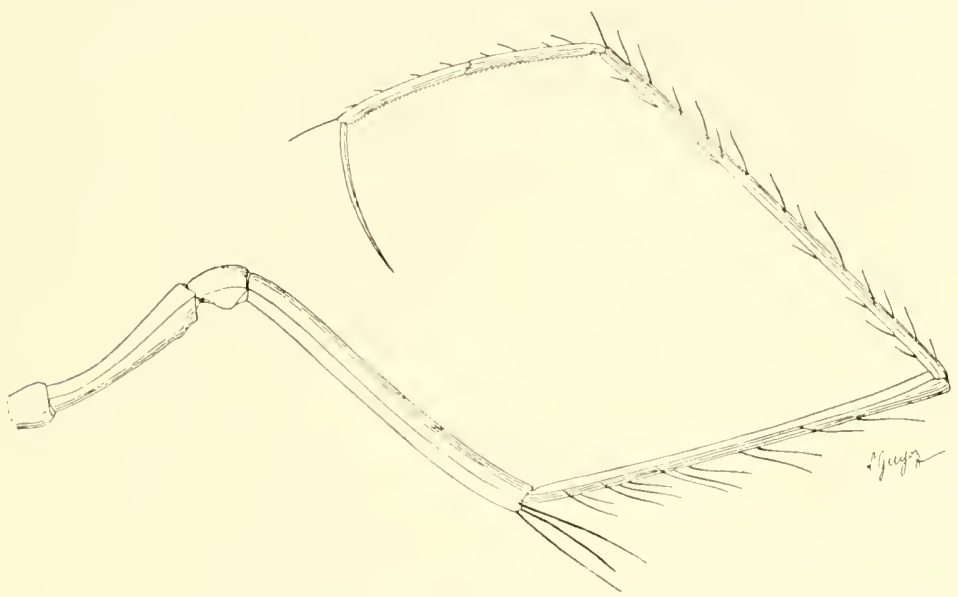

Fig. 41. - Nymphon tenuipes, - Patte droite. Gr. If.

de grilles ausiliares. Il y a dr très longues soies raides et rpaisses sur le fémur et les tibias.

Je nai pu apercevoir les orifices sexuels de sorte fure l'exemplaire est peut-ètre un jeune.

Affinités. - Cetfrespece est frès roisine du N. Iomgicollum IIock (1881, 40, P’. III, fig. I-3; Pl. XV, fig. II) qui s'en distingue : $I^{\circ}$ par son larse court; $2^{\circ}$ par ses grifles auxiliaires qui sont présentes, encore que rudimentaires $; 3^{\circ}$ par le grand développement du deuxiène article des palpes, qui est plus large que les deux suivants réunis; $\mathbf{1}^{0}$ par son lubereule oculaire en còne aigu ; $3^{0}$ par ses grilles plus courles que le propode. Wlle s'éloigne divantage du N. procrenm Itoek (1881, 39, I’l. II, fig. 9-12), donl. le tarse et la grifle sont également bien plus courts que le propode, dont le deuxième article coxal est court et pirilorme, le cou dilaté en arrière du milieu pour l'attache des ovigeres, et dont les pinces sont tries longues et fortement infléchies, la trompe ayant d’ailleurs une autre forme. Le Espedilion Charcul. - Boever, - Pyenogonides lu "Pourquei Pas? w. 
N. Congicollum fut capturé par le "Challenger" au large du Chili, par 2223 brasses, et lo 1 . procemm à l'ouest de Valparaiso, par 2160 brasses.

Nymphon proceroides E.-L. Bouvier.

1911. Nymphon proceroides E.-L. Bouvier (1911), p. ?137.

Dragalge $1^{\circ}$ XVII : 26 décembre 1909 ; Shetlands du Sud, iles du Roi-Grorge, bair de l'Amirauté; latitude sud 62012', longitude ouest P'aristito:3:'; chalut 1, 420 mitres; température de l'eau sur le fond $+0^{\circ}, 3$; vase, cailloux.

Trois exemplaires femelles ( No 724 ) qui se trouvaient noyés dans la masse des $\lambda$. stylops.

Cinq màles et sept femelles (No 732) d'un "brun pâle ".

Je peleve ci-dessons los dimensions d'un male el d'une femelle types:

\begin{tabular}{|c|c|c|}
\hline & o & 8 \\
\hline 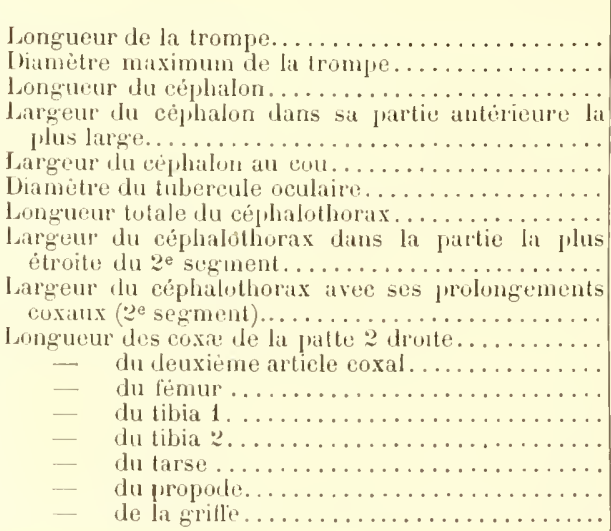 & $\begin{array}{l}\text { Millim. } \\
1,2 \\
0,50 \\
1,30 \\
1,9 \\
1,35 \\
0,27 \\
3,1 \\
0,38 \\
1,9 \\
2,3 \\
1,4 \\
2,5 \\
3,1 \\
0,0 \\
1,1 \\
1,1 \\
1,2\end{array}$ & $\begin{array}{l}\text { Millim } \\
1,3 \\
0,7 \\
1,7 \\
1,0 \\
0,40 \\
0,50 \\
4,20 \\
0,50 \\
2,35 \\
3,2 \\
1,0 \\
3,2 \\
4,7 \\
3,3 \\
1,75 \\
1,45 \\
0,7\end{array}$ \\
\hline
\end{tabular}

Lus pattes de ce l’ycnogonide sont moins grôles que celles de l'espèce précente, mais le corps a une structure analogue.

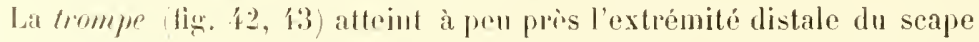
des chólicères; elle se rétrécit un peu vers la base et devient ensuite à pen pries alindrique. 
Lecéplualon fig. 12, 13) est moins allongé que le trone; sa partie antérieure est très dilatée, son con étroit et long; lout à fail en arrière s'élève le tubereule optique, dont la hase est aussi latrge que la partie la plus élroite dn eon, la hanteur assez grande et le sommet obtus ; le tubercule se rétrícil un peu depuis la base jusqu’à là région oenlaire; les youx sont réduits

"et largement séparés sur la ligne médiane.

Le trone (lig. 12, 43) est étroit; ses articles sedila-

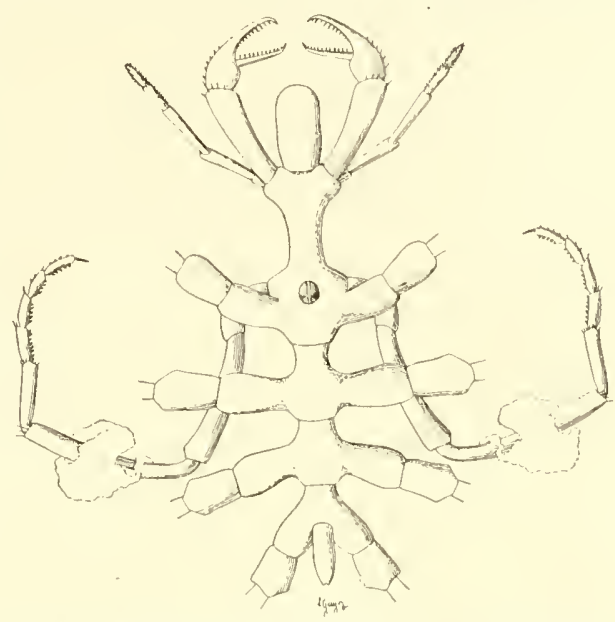

Figr. 12. - Nymphon proceroides bouv., ơ, - Lanimal avee ses chricieres, ses palpes, ses ovigires et la base des pattes flace dorsale). Gir. 8 .

tent d'avant en arriòre et émettent des prolongements eoxamx ì peu près anssi longs que lo serment qui les porte : assez fortement rétrécis vers la base, ces prolongements devienment ensuite subeylindriques (femelle) ou se dilatent légìrement en massue (màle), ce qui présente d’ailleurs quelques variations daus l'un ef l'antro sexe; ils sont séparés par des intervalles aussi grands ou plus grands que leur largeur maxima. Les artieulations segmentaires sont fort nettes.

L'abdomen est eylindriforme,

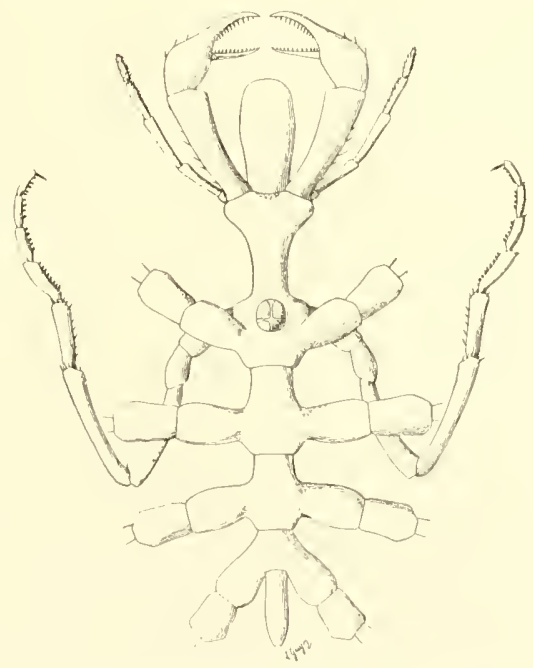

Fig. 43. - Nymphon proceroides Bouv., o*. - Lianimal avec ses chelietres, ses palpes, ses ovigeres et la base des pattes, race dorsale. Gi. 8. 
horizontíl, à sommet obtus; assez long, il dépasse notablement l'extrémité distale du prolongement coxal postérieur.

Les rhélicères (fig. 12, 43 sont assez fortes. Leur scape se dilate un peu de la base à l'rxfrémité distale ef se recourbe un peu en dedans. Les pinces sont à peu pròs de la longueur du scape; leur portion palmaire sulılohuleuse, ouplutot fortement ovö̈de, est beancoup moins longue que les doigts. Ces dernier's sont régulièrement infléchis de dehor's en dedans; lemr armature se compose d'un peigne de longs denticules spiniformes largement séparés, sans armature intermédiaire plus petite.

Les pulpes fign. 12, 13) n'atteignent pas tout à fait l'uxtrémité des pinces; larticle 3 est à peine plus long que l'article 2; l'article í égale les deux tiers de l'artiche 3 ; l'article :iest plus court, plus ćtroit et presique atténué en pointe. Longueur des trois dernier's articles dans la femelle type : $0 \mathrm{~mm}, 73-$ $0^{\mathrm{mm}},: 00,-0^{\mathrm{mm}}, \mathbf{1 0}$. Il y a quelques proils dressés et courts sur les deux derniors articles.

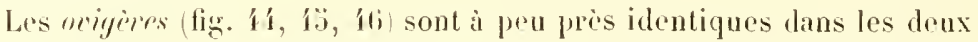

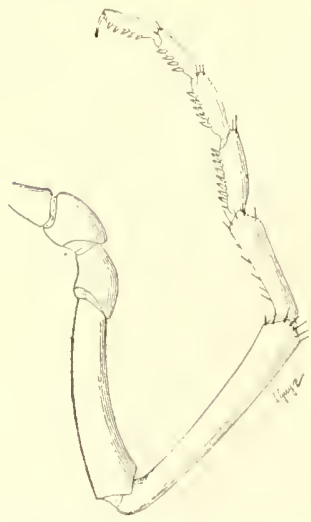

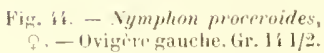

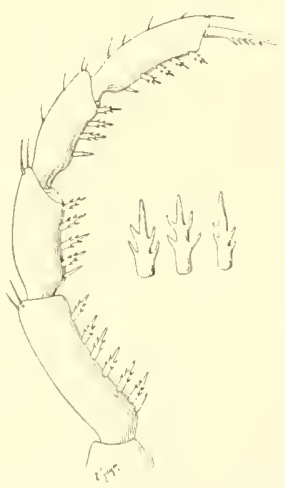

Fig. 45. - Nigmphon procerwites, ơ. - Extrintis de lisvigire droit (Gr. 36) et trois de ses énnes, Gir. 100 .

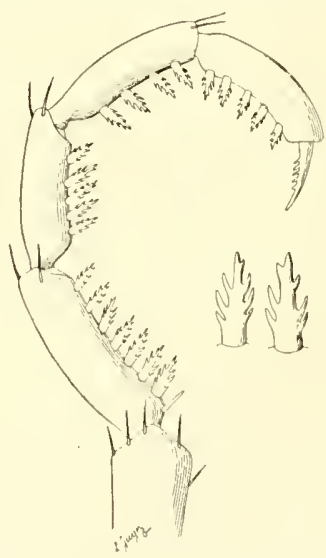

Fin. 16. - Nymphon proreroides, C. - Extrentite le lovigire droit (Gr. 36) et deux de ses epines. Gr. 100.

sexes; leur atticle fi égale la moitié de l'article f, qui est hui-même égal aus trois quarts du cinquieme; ces deux derniers articles sont un preu 
arqués. Les articles décroissent un pen en longueur de l'ardicle 6 an dernier, qui porte une grille étroito presque aussi longue que hui-mème.

Longueur des quatre derniers articles avec le nombre do leurs épines denticulies dans lo type formelle firg. (1ti):

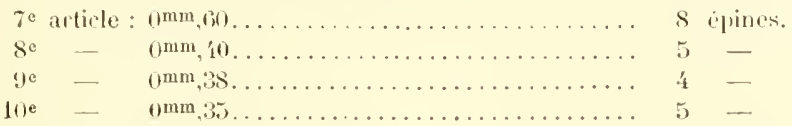

Les pattes fig. fí-̈ol se distinguent par lit póduction de leur second

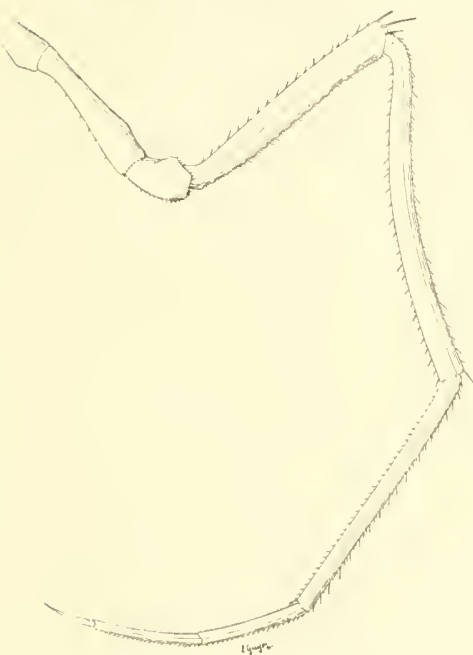

Fig. $\mathbf{k}$. - Nymphon proceroides Buuv., O'. - La ge patle droite. itr. $61 / 2$. tilia, qui est à peu prìs de la même longueur que le fémur et notableunent plus court que le tibia 1; leur tarse est ézalement un peu phus court que te proprode; les grifles auxiliaires font totalement défaut. Le deuxieme arlicle coxal est à peu près aussi long que les deux autres articles coxaux; il présente dorsalement une

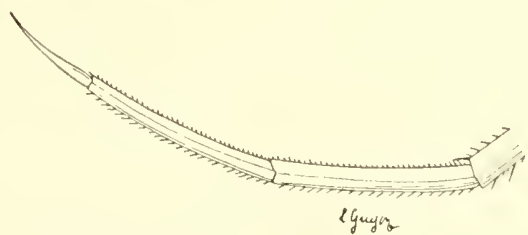

Fig. is. - vimmphon proceraides o". - Les trois artiles tirminaux de la 20 palte droite. Gi. I6.

saillie subdistale sur laquelle je n’ai pru voir des orifices glandulaires. Cet article se dilate un peu do la bise au sommet ehez le mâle, tris fort, ot de manière à devenir piriforme chez la femelle lig. iz, où d'ailleurs le fómur est heaucoup plus penflé que chey le màlr. Il y a un orifice sexuel sur toutes les pattes dans lit femelle, ef l'on voit, chez certains màles, unc rangée de saillies à jores cémentaires sur lo bord ventral du fémur.

Le copps est dipourvu de pubescence, mais très finement granuleux ; les pattes sont cilires dre quelques soies fort courtes. 


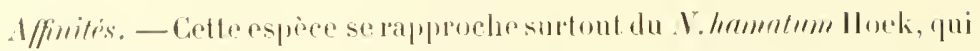
s'pn distingue d'ailleurs par ses yeux ubsoletes, ses pinces beaucoup plus longues, plus atruées ef plus fortes, ses prattes plus allongées et plus

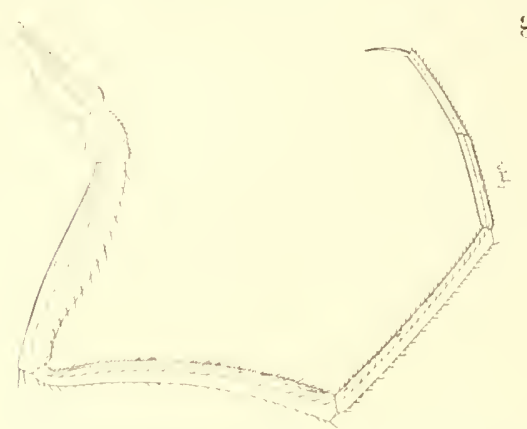

Fin. 49. - Nymphon mocerobles, P. - La patle sauche i fartir du 2 article rosal. fir, $i$ i/s. gricles, ses palpes où l'article 2 rst

beaucoup plus long que l'article 3 ; il mpelle le 2 . proremm IIock par la dilatation (chez la femelle) dusecond article coxal; mais tous ses atutes

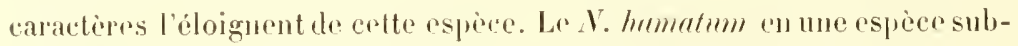
antaretique trowvée pas le "Challenger" dans les parages des ites Crozet,

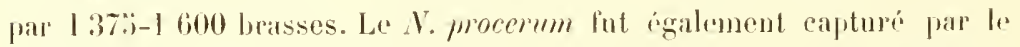
"Challenger"; c'est une espece abysale trouvée à l'onest de Valparaiso.

\section{Genre Chætonymphon R. O. Sars.}

Le genere C'hirtomymphon se rattache étroitement aux Pymphon, dont il so distingue par le corps trapu, la pilosité abondante et la brieveté relative des pinces des chélicères. Les 13 espèees qu'il renferme sont toutes localisées au voisinage des priles et descendent rarement it des profondeurs un peu grandes; 8 d'entre elles sont antaretiques of caralérisées par lenr tarse plus longr que la moitié du proprode; les :̈ autres habitrut les mers aretiques et se distinguent par leur tarse court, qui alteint au plus la moitié du proprote.

l'ne esporer du premier groupe, le Ch. hrevicaudutum, a été recueillie par les maturalistes du "Pourquoi Pas?". 


\section{Chætonymphon brevicaudatum Miel's.}

1875. Mymphon brevicaulatum E.-J. Miers (1875), 1. 117.

1879. Nymphon breviraudatum F.-J. Niers (1879), 1. 2016-21', PJ. XI, lig. 8.

1879. Nymphon horrilum R. Bälum (1879), 1. 175, Taf 1, lim. 3.3 f.

1881. Nymphon breviraudatum P.P. C. Ilock (1881a), p. 49), Pl. IV, lig. 13-13, et I'. V, lig. 1-5. ( 1 . hispidum lans les planches).

188s. Nymphon brevicaudatum G. I'feflep (1888), 1). 41, I889).

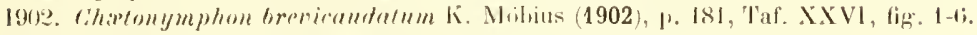

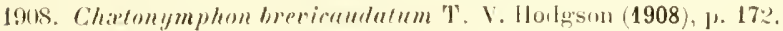

1910. Chatomymphon brepicantatum E.-1. Bouvier (1910"), 1'. 178.

19]1. Cikietonymphon brevicautatum E.-L. Bonvier (1911"), 1). 1138 (1911"), p. Ali.

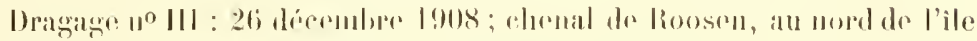

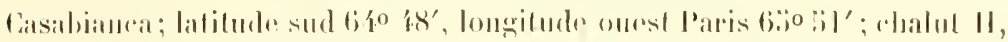

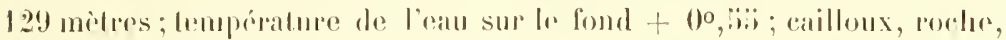

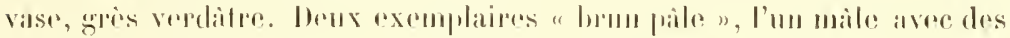

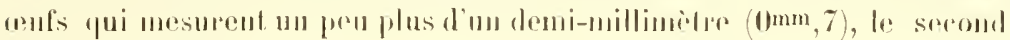
fontelle $(10000)$.

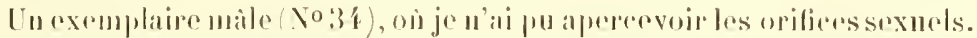

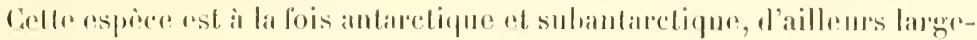

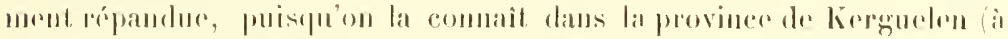

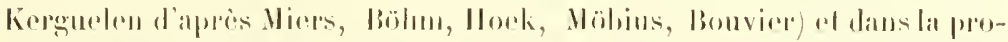

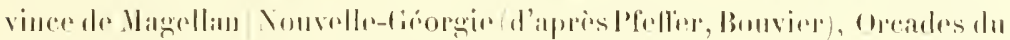

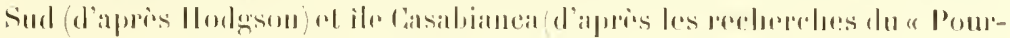

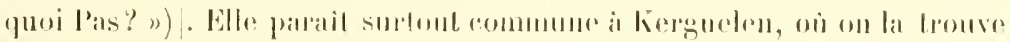

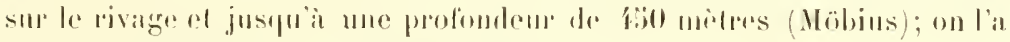

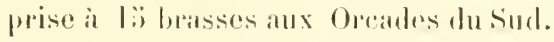

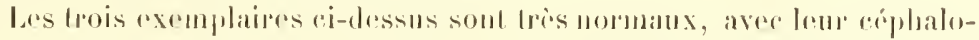

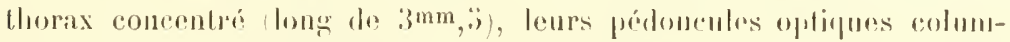

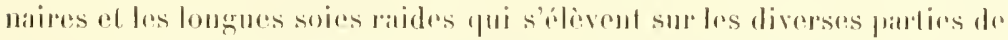

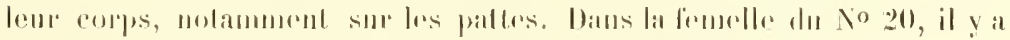

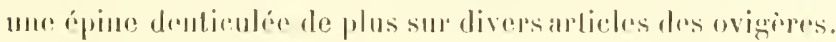

Deuxiòme Fanille. - PALLENIDE 1'. P. C. Hock.

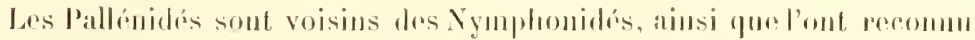

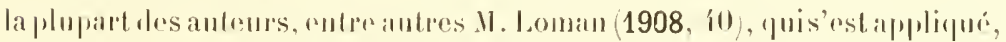

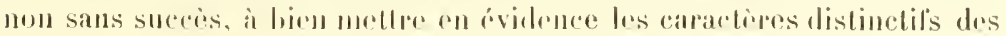


denx genres principaux de cette famille: les l'alleme Johnston et les Parupellen Carp. Les palpes font presque tonjours déant chez les Pallénidés, mais on en tronve rncore des rudiments dans quelques especes de

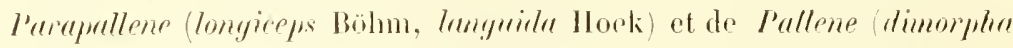
Hork, ralida Hasswril . de même que chez les mâles du genre Veopullone [Dohrn. Ces rudiments se rédnisent à un court bourgeon, sauf dans lì l'arapallene longiceps, on ils sont anssi longs que la trompe et comptent denx articles, ainsi que j'ai pu le constater sur un exemplaire des colleetions du Muséum (1911a, 1136). Les ovigìres sont également un peu variables: d'ordinaire, ils ont une structure primitive et se composent de dix articles, arec épines différenciées el griffe terminale; pourtant la grille manque à tous les Pallene, sauf dimormhla Itock, et à deux espèces de I'rraprallene (ongieeps? et Innguila); il n'y a pas d'épines spéciales aux ovigires des Parapallene capra Loman et Gimbei lloek.

La famille comprend cing genres : Prumallene Carp, Cordylorkele G. 0. Sar's, Psendopallene Wilson, Pallene Johnston et Verpallene Dohrn. Lestrois premiers se distinguent par l'absence de grilles anxiliaires, fandis que les deux autres en sont pourvus. Dans le genre Parapallene, les doigts des chélicères sont finement et régulirrement denticulis, comme dans les Nymphonidis, alors que les épines sont remplacées par de grosses dents sonvent alssentes chez les Cortylochele el l'spulopulleme, les expices dece dernier genre ayant sur le corps, ordinairement aussi sur le scape des ehélicires et sur les pattes, une arnature épinemse pui manque aux gemes précádents. Guant aux Pallene, elles se distinguent des. Teopallene par leurs ovigieres, qui sont presque toujours dépoursus de grifles ef dont les fines spéciales sont obtuses au lien d'itre aiguës.

Ainsi comprise, la famille compte actuellement 31 espèces, dont :i seulement sont antaretiques, la Pallenedimorplua llook, el f́ espeees du genre I'sezulopullene. Le nombre desappiees archipuesest de 9.

Les trois espieces recueillies par le "Pourgmoi l'as? " appartiement tontes an genre Psemlopullene: deus dentre olles neétaient pas eomnues juscu'alor's. 
Genre Pseudopallene Wilson.

Ce genre est propre aux rógions polaires; des 6 rspèces qui le représentent, 2 labitent les mer's arelipues $I^{\prime}$. spinipes fab. et $P$. circular is Goodsir), les $i$ autres sont antarctiques. A l'exception de la P'. anestrelis Hodgson, ces deruieres font partie des récultes du "Pourpuoi l’as?"

Les l'seudopallene sont très voisines des topdylochele, qui comptent 3 espèces toutes arctiques. J'ai indiqur plus haut p. !(ti) les caracteres essentiels qui différencient les deux genres; il y aurait anssi, chez les /'sendopallene, des soies menues autour de la bouche, mais il ne m'a pas été possible d'observer ces formations spéciciles.

\section{Pseudopallene cornigera Möhius.}

1902. Pseudopallene cornigera K. Mobius (1902), p. 1886, Taf. XXYll, fig. 14-20.

1905. Cordylochele Turqueti E.-L. Bouvier (1905), p. 297.

1906. Cordylochele Turqueti E.-1. Bouvier (1906'), p. 18.

1900. Cordylochele Turqueti E.-L. Bouvier $\left(1906^{\mathrm{h}}\right)$, p. 33-39, tig. 7-18 bis.

1907. Pseudopallene cornigera T. V. Hodgson (1907'), 1. 7. Pl. 1, fig. 3.

1911. Pseudopallene cornigera E-L. Bouvier (1911 ${ }^{\mathrm{b}}$ ), p. 1138.

Dragage ${ }^{\circ}$ XVII : 26 décembre I909; Shellands du Sud, ile du lioi-George,

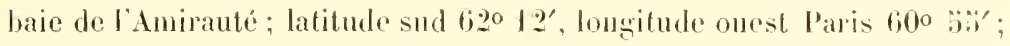
chalut 1, 120 mètres; température de l'ean sur te fond $+0^{\circ}, 3$; vase, cailloux.

Un exemplaire "brun "sur le vivant ( $n^{\circ}$ 73.4), anjourd'hui tont a fait décoloré. Coest un mâle parfaitement adulte, encore qu'il ne nnat pas élé possible dy apercevoir les orifices sexuels. Virs le bout distal du long article des ovigères (le cinquiène), se frouverncope lenanchon cémentaire qui réunissait les ceufs, et des larves sont enrore agrippres aumantelon. Ces larves nont fue deux paires de pattes bien diveloppées, la troisiome étant réduite encore à des bourgeons; les chélicires y sont puissantes; la tromper est réduite.

Cet exemplaire ressemble lout à fait à colui que j’ai désigné andé.ienrement sous te nom de Cordylurhele Turqueti, notamment par sa trompe qui est cylindrique dans la moitié basilaile el ensuite franchement conique, par ses prolubérances cóphalipues peu divergentes, par son abdomen franchement horizonfal ef par les saillies à peine indiquées de ses prolonExpédition Charcot. - Bourien. - Pycnogonicles du "Pouryuoi Pas?". 
gements coxaux; ta seule dillérence, èest que les ovigìres ne se terminent point par un petite grille, mais par une épine denticulée. Il semble donc bien que les deux exemplaires dus à M. Chareot se distinguent de l'espècen de 11. Möbius. Pourtant its ont avec elle les ressemblances les plus itroites, et je pense qu'il est sage de se rallier à l'opinion de M. Ilodgson qui tend à identifier les deux formes. Toutefois, comme on vient de le voir, il s'en faut que cette identité soit alsolue ; l'espèce a yant été capturée dans la province de kerauelen (aux îles Bouvet, par fö0 mètres, d'après 11. Möbius), dans la province austrakienne entre 12:3 et il brasses d'après 11. Hodgson, puis par les deux expéditions Chareot dans la province de Magellan (Port-Chareot et Shetlands du sud), on peut croire qu'elle présente des variations assez importantes et que les exemplaires de la provine magellanique sont notablement dillérents des autres.

En tout cas, je crois que cette espece établit un passage très net entre les p'seudopallene et les c'mdylorlecte et qu'il ne convient pas de distinguer les deux genres par la présence des soies buceales, car je n’ai pu observer ces dernières dans les deux spécimens rapportés par M. Charcot.

\section{Pseudopallene brachyura E.-L. Bouvier.}

1911. Pseudopallene brachyura E.-L. Bouvier $1911^{\mathrm{b}}$ ), p. 1138.

Dragage no VI : 1 3janvier 1909, entrós de la baie Marguerite, entre l'ile Jenny et la terre Adélaïde; chahut I, 204 mètres; te mpérature de l'eau sur le fond $+1^{\circ}, 18$; roche, gravier.

Un exemplaire femelle "jamme pâle " (No 136$)$.

Voici les dimensions de cet exemplaire:

\begin{tabular}{|c|c|}
\hline & \\
\hline n de la trompe (au milieu). & 0,4 \\
\hline halon................. & $1, ?$ \\
\hline 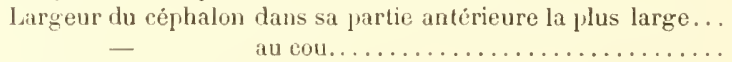 & $\begin{array}{l}1,5 \\
0,4\end{array}$ \\
\hline 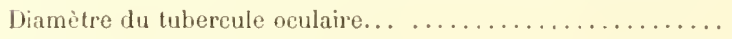 & 0,4 \\
\hline 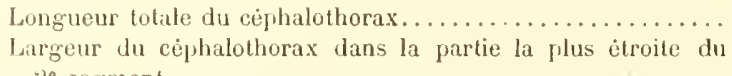 & 4, \\
\hline uts $(20$ & \\
\hline
\end{tabular}




\begin{tabular}{|c|c|c|c|c|}
\hline & 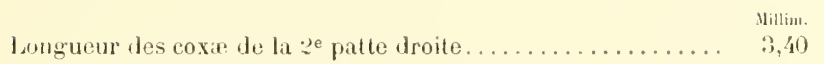 \\
\hline 一 & du $2 e$ art. coxal & - & $\ldots \ldots \ldots \ldots \ldots \ldots \ldots$ & 2,001 \\
\hline & fémur & - & $\ldots \ldots \ldots \ldots$ & 4,5 \\
\hline- & tibia 1 & - & $\ldots \ldots \ldots \ldots \ldots$ & 1,0 \\
\hline- & tibia 2 & - & $\ldots \ldots \ldots \ldots \ldots \ldots$ & 5,2 \\
\hline - & tarse (fort cour & le 1 & 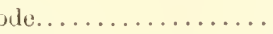 & 1,9 \\
\hline- & de la griffe... & & $\ldots \ldots \ldots \ldots \ldots \ldots \ldots \ldots$ & 1,2 \\
\hline
\end{tabular}

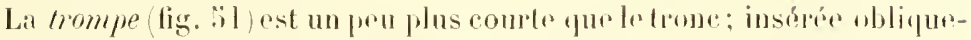
ment el ventralement en arriape du bord anterirur de la parlie cóphalipue, olle s'étrangle notablement an nivean de son premier lirers. puis se dilate un peu et se rétrécit en còne terminal. Sa pointe buecale, très légrimement dilatíe, porte quelyues menues saillies mais me semble pas ormée de soies.

Le criphalon (fis. : 1 , :i2) est médioerement allongé; il so dilate mu peu au nivean du tubercule optique, afin de donuer maissance aux ovigieres, phis vient un cou fortétroil ol nne rógion céphalique tries large qui se prolonge en dessus, de chanue coté, en une pointe dirigée obliquerment en dehors. Le tubercule optipne est, it sa base, aussi large que le cou, peu éleré et large-

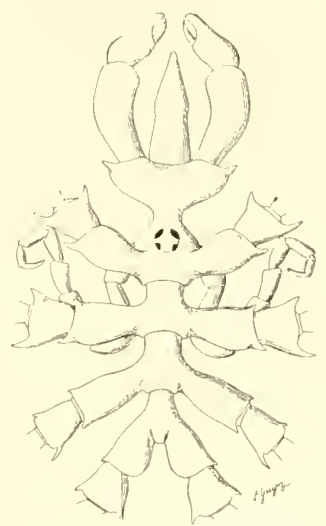

Fig. 51. - Psendoinallene bra-

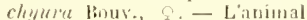
vu de des a vere les chiliceres. les ovinimes et la basedes palles. Gr. 8.

ment obus; il porte quatre yeux d'un noir rougeatre hien séparés et assez pedits, les denx antédicur's ćtaul d'ailleurs plus grands que les autres.

Les segments du trone fig. $.11,92$ sont pen larges, nettement arliculés et

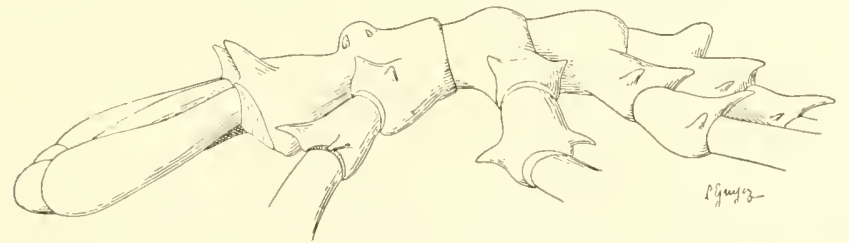

Fig. 52. - Pseudopallene brachyna, Q. - Lexemplaire de la figure próribente vu du collé gauche, avec les chidicires el la base des palters. Gr. 16.

rétrécis en avant; leurs prolongements coxaux sont assez longs et sriprés par de grands intorvalles; rétrécis ì la base, ils se dilatent rnsuite légire- 
ment of se teminent par deux pointes, l'une situce en avant, l'antre m arrière.

Liublomen se réduit à une légire saillie subeonique, d'où le nom de brachyura quo jattribue à l'espece; il est à peu près vertical.

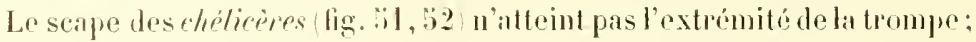
il se recourbeen dedans et se dilate assez fortement de la base au sommet. Les pinces sont relativement rídnites, un peu phs comrtes que le seape,

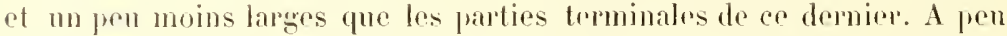
pris droites, coest-à-dire sans inllexion, olles se distinguent par leurs doigts qui sont dípourver de dents et à peu pròs de la longueur de la portion palmaire. Le doigt mobile esl grêle, pen arqué el porte une grille aignä; le doigt lixe rst beaneomp plus large, bien droit, et se termine sans grille par un bout largenent ohtus.

Les ovigimes fiz. : il, :33 sont forts of assez courts; l'article 2 est plus

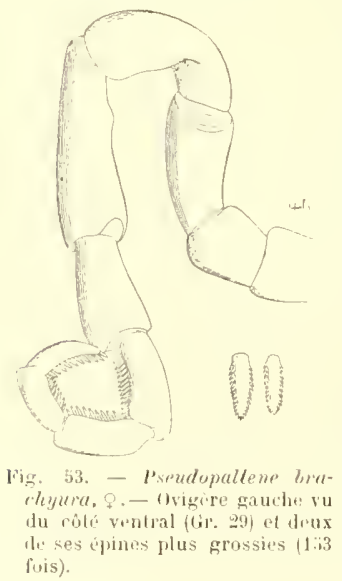
lons que le premier, et le troisieme ì pen pres de môme longneur que les deux précedents rénnis; l'artich.' dépasse d'environ un tiers la longueur du troisicme et égale le suivant. mais il est arque; les autres articles sont subégaux et se réduisent de phus en plus on largenr. On observe mo sallie sme lo bord de l'arliele :i.

Les épines dilfrenciées des quatre derniers articles porlent des denticules nombreux, mais pron saillants, qui premment une forme linéaire dans la partie terminale. L'état desovigires me mat pas permis de fixer le nombro des épincs, qui paraissent assez nombremses. Celle qui ocenpe le bout du demier artirle ressemble

it moe suille drutio.

Les pulles, fign. : if sont remarquables par les deux fortes pointes distales de lour premier article coxal, parla forme et la dimension do l'article z yui

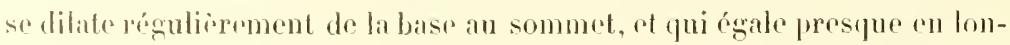
gueur la moitié du lómur. Ce dernier est très dilaté dans notro type quui est une femelle, a peine phus long que le tihia 1, mais beaucoup phus 
court que le tibia 2. II y a de fortes soies spiniformes sur te bert interne du tarse, qui est très court, ot du propode, yui est normalement arrué; la grrilfe est presque aussi longue que ce dernier article. Le deuxième article coxal (lig. 3ii) présente quelques faibles saillies coninues terminées par une courte soir raide; des saillies plus réduites mais plus nombreuses of longitudinalement situées se troment sur les deux tibias, où elles se terminent aussi par de courtes soies; les soies fémorales sont rares, plus réduites et sans saillie basilaire.

Les orifices sexuels, grands et

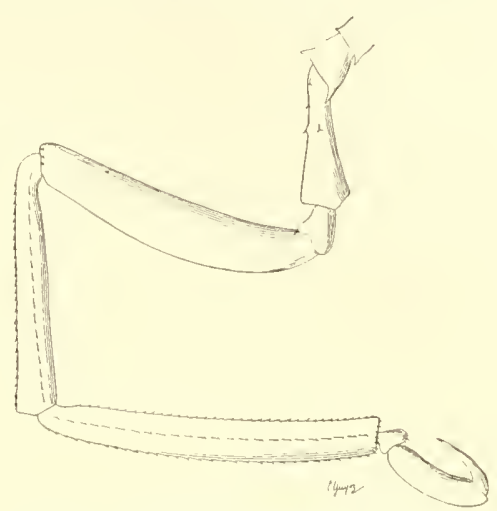

Fig. 5i. - Pseulopallene brachyurt, +. - La 9: palte gauche (un n'a jas figuré les soies du tarso et du jropode). Gl', $61 / 2$. ovalaires, se trouvent sur la face ventrate etprestue à l'extrémití distale de tons les denxirmes arlicles coxaux.

Iffinités. - Cette aspèce est voisine de la P. australis ILodinson

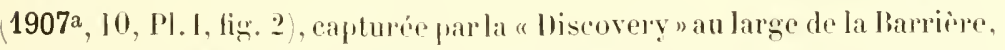
latitude sud z $8027^{\prime}$, longitude est Greenwich 18:30 39', ì 300 brasses dr profondrur. Les caractipes qui distinguent cotte demiere esprece de la notre sont les suivants : fo la trompe ne présente pas de rétrécissement dans sal moitio basilaire: 2o la partic antérieure du céphaton est relativement beaucoup plus large: 30 l'abdomen est assez long. fusiforme et obliqurment relevé fo les saillies forminates aiguïsdes prolongements coxaux et du premier article cosil sont beancoup plus priduites; ;o la pince des chélicires est beaucoup plus large que le scape, et son doigt fixe se trimine en pointe; 6o l'article $;$ des ovigires es beaucoup plus long que le précédent, et les ŕpines dillárenciées des quatre articles terminamx ne sont denliculées que dans leur moitié distale; $7^{0}$ l'article tilial 2 des pattes n’est pas plus lonğ que le précédent. Les saillies sétifères des patters sont à peu près distribuées dr même dans les denx esprices. 
Pseudopallene cristata E.-L. Bouvier.

1911. Pseudopallene rristata E.-L. Bouvier $\left(1911^{\mathrm{b}}\right)$, p. 1138.

Dragage no V : 29 décembre 1908; chemal Peltier, entre l'ilot Groetschy et l'ile Domner; phalut II, 92 mètres; température de l'eau sur lo fond - 0o, 1 ; vase grise, gravier.

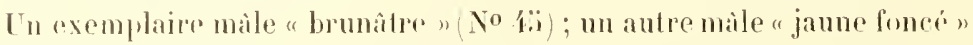
avec des reulis (No 17 ).

Dragage $1^{\circ}$ VII : 16 janvier 1909 ; pres de la Terre Nexandre; chalut I, z:i0 milres; température de l'ean sur le fond - 10,6; roche.

Un exemplaire màle " jaune sal, " avec des larves (No lï). Voici les dimensions de ce dernier exemplaire:

Millin.

Longueur de la trompe..................... 2,6

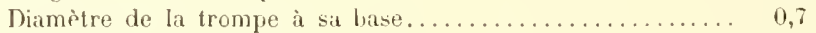

Longueur du céphalon..................... $t, 7$

Largeur du céphalon dans sa partie antérieure la plus large.. I,9

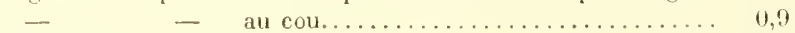

Diamètre du tubereule oculaire.................. 0,1

Longueur totale du céphalothorax.............. 4

Largeur du céphalothorax dans la furtie etroite du 2e segment. $\quad 1,3$

Largeur du céphalothorax avec les prolongements latéraux

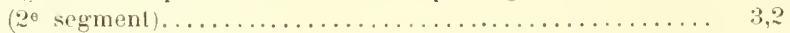

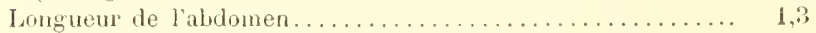

- des coxe de la $2^{e}$ palte droite.............

du $2^{\theta}$ artiele coxal.....................

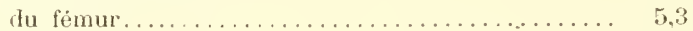

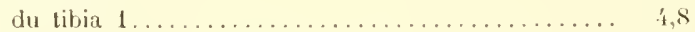

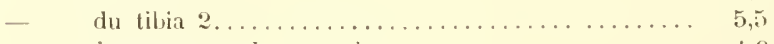

- du tarse avec le propule.................. 1,9

- de la griffe........................ 1,3

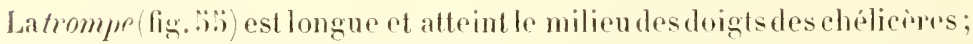
"llescrétréritun pen depuis la base dans son premier tiers, beaucoup plus ensuite et réguliorement pour se terminer en m bord ctroit et oblus, de teinte brunâlre, yui m’a semblé dépoury de soies.

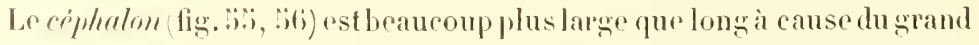
développrment drsa partie antérieuretpuisecompose, commed'ordinaire, de deux lohes; wacun deces derniers so jrolonge en une courte pointedorsale. Is eou ast un simple étranglement. d'ailleurs probond: en arricre, le 
ciphalon se dibite un pen et prote le tubereule oculaire, qui est un mamelon arrondi an sommet. Les yeux sont noirs el assez grands, surtout ceux de la paire antérieure; des introvalles très nets les séparent.

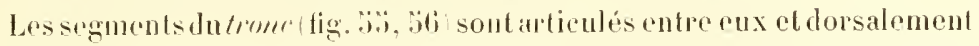
saillants, surtont an bord postérieur, où ils s'ćlevent en une forte et large pointe inclinse en arriere. Les prolongements coxanx sont presque contigus, étranglés il la base of a preine aussi longs que la largeur le l’article; sur chacun de leurs angles dorsaux externes s'blivent une ou denx fipines

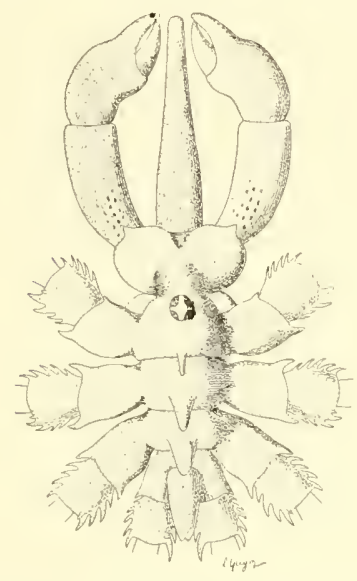

Fig. :ij. - I'seudopullene crislata lious. - Un exemplaire of vu de dos avee les chélicires el la base des palles. Gr. 8 . recombresen dehurs et semblables à celles que nous trouverons en abondance sur los pattes.

L'abdomen fign. (i.i, Gifi) est assez lonš, fusiforme et notablement televí.

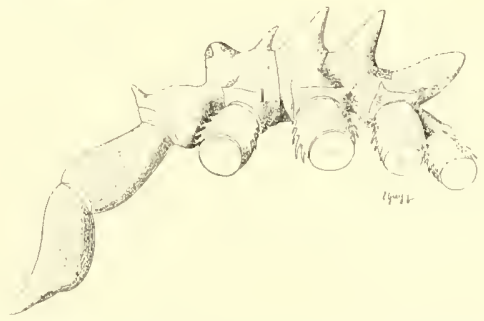

Fig. 56. - I'seartsprltene cristata. - In ó vu du cóte gaucle, avec la chelicere correspondante. Gr. 8 .

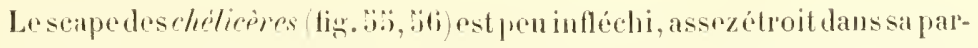
tiebasilaire, rnsuitebien plus large el subeylindrique. Lespines, peupuissantescetsans grande inflexion, présentent a pen pris la Jongueur du seape; assez étroites à la base, elles offent leur largeur maxima un peu avant la base des doights; ces dernierss ont à peu jrès la longuerur de lat portion palmare; leurs bords internes sont incurvis; leur pointe brunr est subaiguä; le doighmobile est légèrement arqué, le doight fixe presłue droit. II y a quelques faibles denticules sur la face dorsale du seaje des chélicires.

Les ovigères (fig..:fi) sont tris remarquables parla longucur et la forme de 
leur cinquirme article, qui est aussi long que les quatre précédents réunis, grèle et bacilliforme dans tonte son étendur, saufà sa partie distale, où il s’é-

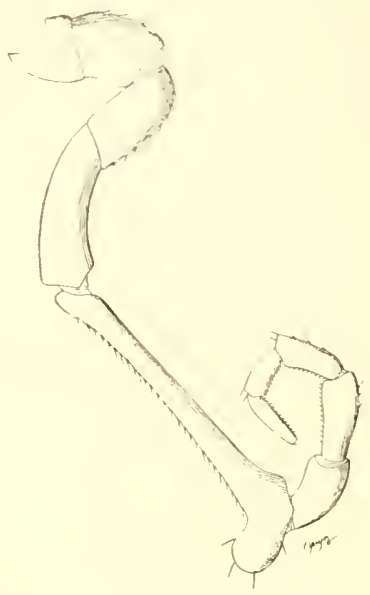

Fir. 57. - Pseudopallene cristata, o: - Origire du ecilé droit, iu par la lace dorsale. Gr. $+1 / 2$. largit hruspuement et porte en outre une shérule sursa face externe. Le premier article est tres court: le deuxième notablement moins; is

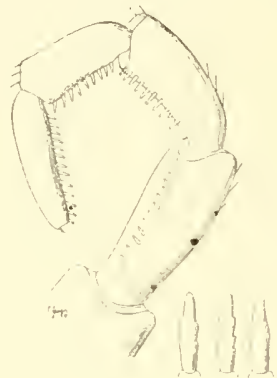

Fi̊n. 58. - Pseudopallene cristata, 0* - Extrémité d'un ovigire (Gr. $3 \mathbf{t}$ ) el trois de ses épines plus grosisies. lir. 153.

troisième à peu près aussi long que les deux précédents réunis, mais plus court que le quatrieme, qui est arqué en sens inverse: les cinq derniers articles sont subégaux (fig. :̈. Les épines différenciées ont un bout obtus et portent tries peu de denticules ou pas du tout, comme on l'observe notamment sur celles des septième et huitième articles; à l'extrénité distale obtuse du dixième article jai olsorvé une très légère spinule dans le spécimen dont j'ai fait me étudr approfondie. Dans cet exemplaire, les épines denticulées des articles $7,8,9$ et 10 étaient au nombre de $13,10,9,13$.

Lis pattes lig. it) sont tout à fait remarpuables par leurs épines serrées, fortes et recourbéesen dehors: sur lo lémur et les deux tibias, ces épines se groupæul rn quatre rangées longitudinales : deux dorsales, une rn avant, l'autre en arriere; il y a en outre une rangée ventrale d'épines beaucoup pluspetites; surle fémur, la rangée dorsale antérieure est incompletenent développée. Il y a simplenent denx rangres latérales sur les articles coxaux, el encore ne sont-elles bien développées que sur les leux pre- 


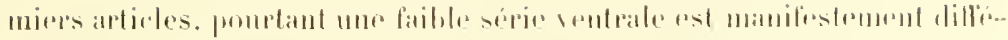

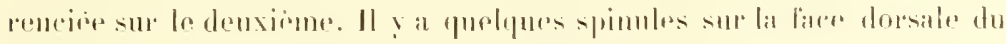
tarse ef du propode. des soies spinilormes sur le borrl interne de ces deus articles. La grilli ex prestgur aussi longue yue lo pro-

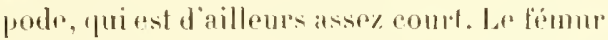
et le tilia 2 soml it pur pris de mêne longuene el notablementplus longs que lelibial. Le deuxioue article coxil est un peu phus long gue les deux autrespéumis ; sur sa face dorsale plane sinlive, à la maissane du tiers distal, un tubercule olitus probabloment grandulaire. Les orifices sexuols, triss dilliciles is apercevoir, mont paru loralisés sur les deux paires dr patles postérieures, où ils ocrupent ventralement le bord distal te l'article. Hianetre des muts, $300 \mathrm{y.}$

Iffrités. - Par le revelement épineus de ses appendices, sa ruite clorsite, la formedu corps et la mature du tuberecule optichue, erotle espece présente des alfinités

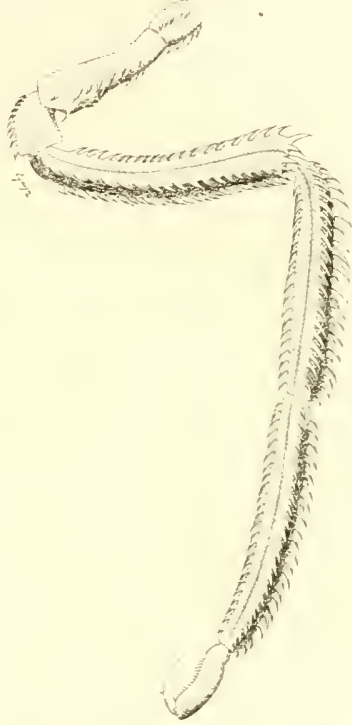

Fig. 59. - Pseulopallene cristatu, - La ge patte droile. (ir. is $1 / 2$

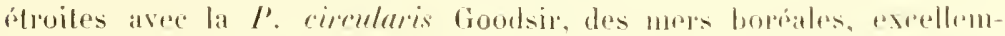

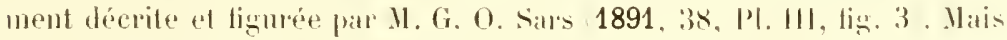
elles'en distingue au premier abond par sa trompe eflilée of nom subeylindrique. les doigts imermes de ses pinces at par ses duberoules dorsaus.

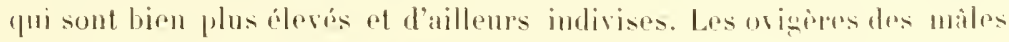
der motre especesont en outere tries particuliurs.

\section{Troisieme famille. - PHOXICHILIDIID}

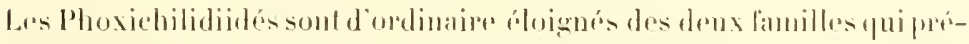

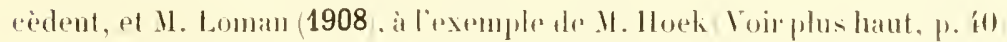

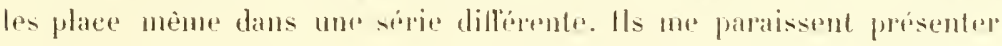

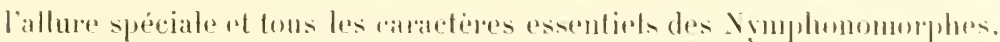




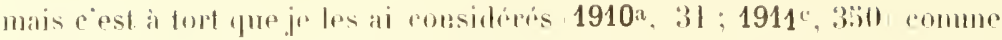

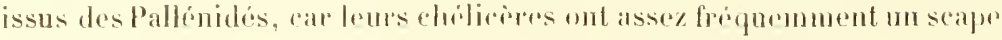
dedeux antichs, et re caractire ancestral fait defaut a tous les Nymphonidés el Pallénidés actmellement commus. En fait, les Phoxichilididés, avec les Phoxichilidés gui lemr font suite, comstituent dans les Nymphonomorphes m ranean parallelo andui des Nymphonides-lablenides.

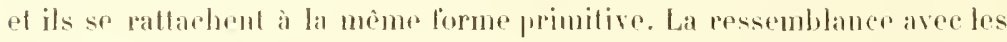
l'allénidés est tros grande palpes rudimentaires ou muls, ovigères sans griffe ni épines comme rhez rortains Pallénidés, mais ello provient d'une évolution analogue, non d'me filiation entre lis drux familles. An surplus, il est mu earactire struetural qui permet de distingure an premier abord les Phoxichilididés, rest la position du tubercule oculaire qui se trouve en arant du miliou ducéphalon au lieu d'être, conme de

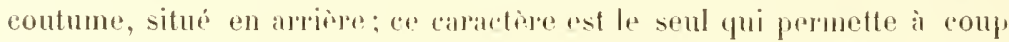
sur de distinguer la lamille, mais il ast le résultat d'une adajtation secon-

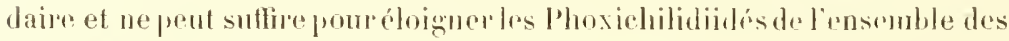
autres Nymphonomorphes.

M. Loman (1908, 633-ti:i) a justement insisté sur l’imprortance el lrs varialions des ovigires tans cette lamille, oi ils sont d'ailleurs foujoms dépoumus dépines spéciales at de soilles: ils se composunt de dix

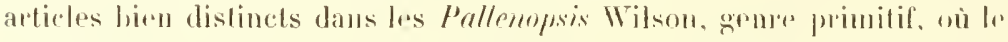

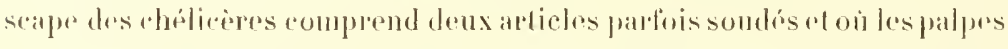
existent encore a l'état de loudiments; dans les Kigomen Loman, qui sont des f'ullemopsis à seape simple et sans palpes, on trower dejà plusiems anticles conerescents aux ovigeres de la lomelle: les ovigeres mantuent chor la linmello dans les trois antros gemors, qui constiturnt le reste do la famille, ceus du male compremant six anticles dams les Lumplorlartylus

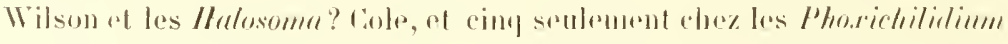
Edw ards, qui nous presentent le terme de l'ívolution chez les Phoxichili-

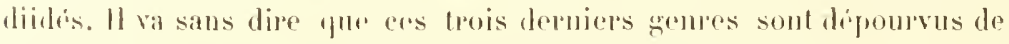

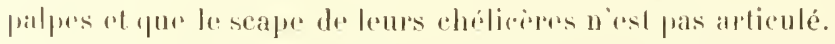

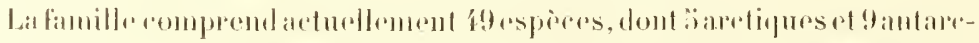

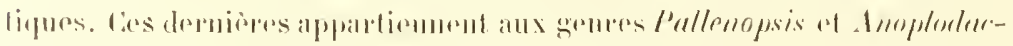

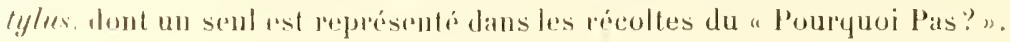




\section{(ienre Pallenopsis Wilson.}

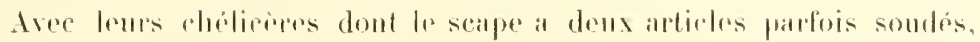
atre lenes palpes rudimontaires, lems ovigèes do 10 ardicles dans les

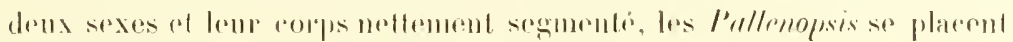

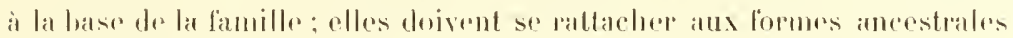

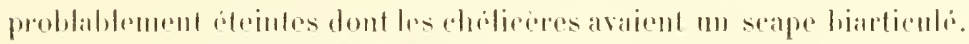

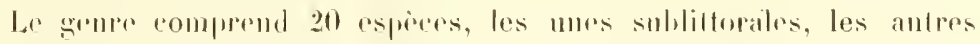

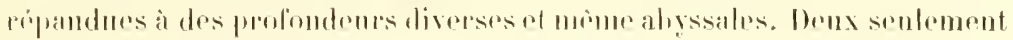
sont arefiques, tamblis quion en connait i dans les regions avoisinant le poile

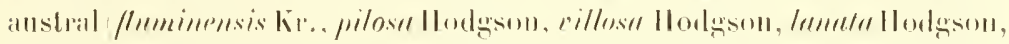

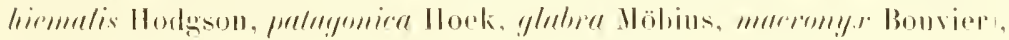
doml hois ont efe recueillios par lo "l'ourpuoi l'as?".

\section{Pallenopsis pilosa lloek.}

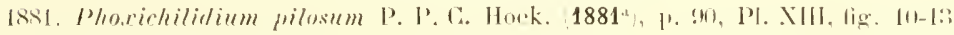

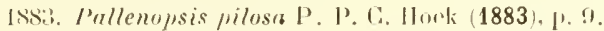

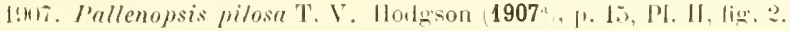

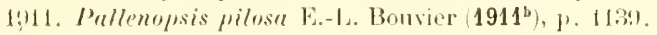

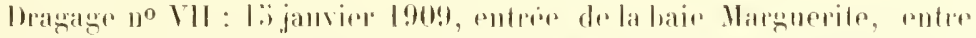

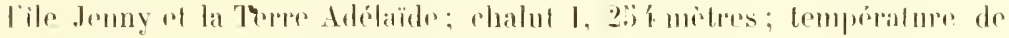
lirau surle fond $+10,18$; roche, smivier.

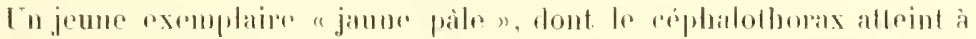

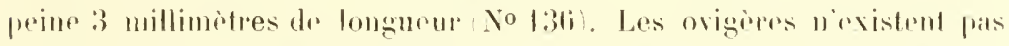

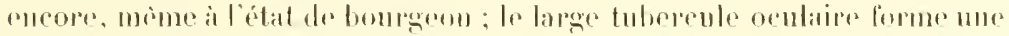

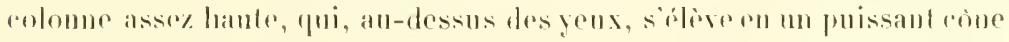

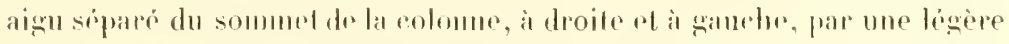

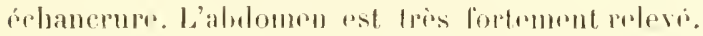

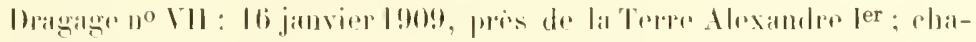

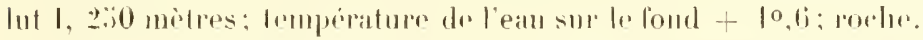

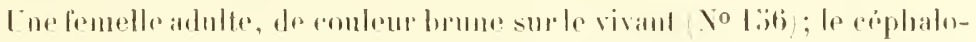

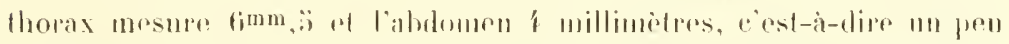

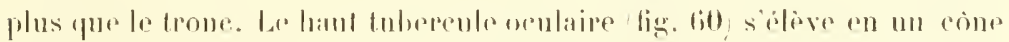

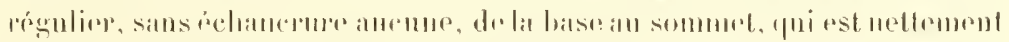

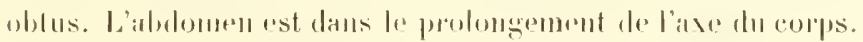




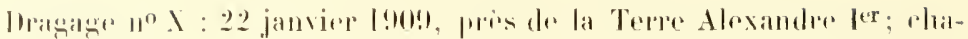

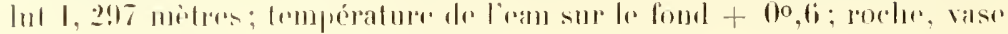

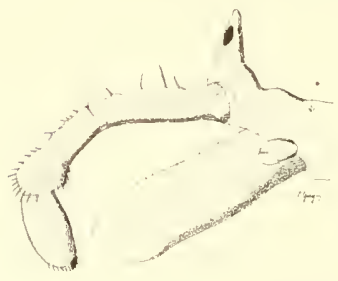

Fig. 60. - Pullenopxis pilosa, f.lartie antirieur. the rephaton aver les appendires du coti

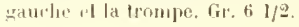
blene. .

Ine lemelle de mome taille of de mime colorations fure la préédente. mais la lu-

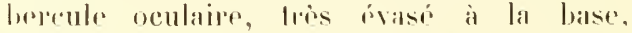
devient presigue une colonme jusqu'au-dessus des yeus, aprers proi il se contimme pal ume saillie arrondie qui prósente au sommen une cicatrice avere un liger mucron. Labdomen est trios lingerement mene (No 283 ).

Comme on vient de le voir, les variations des lubercules aculatres et de liablomen sont assez grandes daus ers exemplaires, qui, d'aillems, par lous leurs autres caractires, se rapportent manifrestrment al'espice de H. Hoek. Il y a strement d'antres variations, ear, d’apris M. Ilodgson, le fémur of le tibia I sont de longuem inale daus les spreimens de la "Discovery", alor's que le seound de ees articles est mobablement plus court que

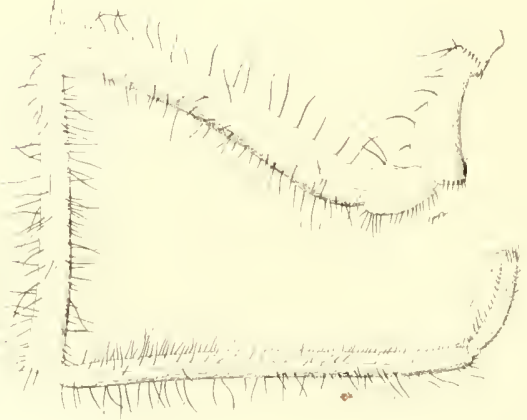

Fig. 61. - Pallenomsis pilosa, \&. - La ge patte siache. Gr. \& l'autre daus nos exrmplaires (tig. 61). Napres lo meme auleur, lrseapedeschélicères esl long el formí diun seul articke, mais il présente du coté dorsal un charissement submédian qui indique la fusion possible de deux arlictes; M. Hork n'a point siznalé rette fusion et, dans le texte comme dans les figures, signale deux articlirs imlependants: nos exemplaires fig. 60 tirmont le milien entre ceus

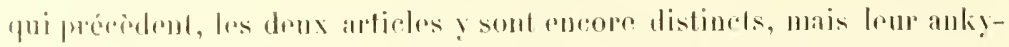

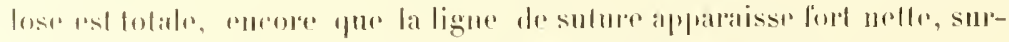

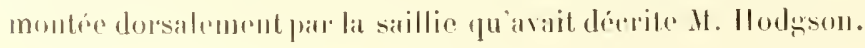




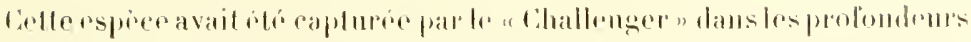

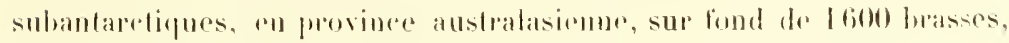

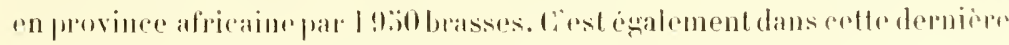
provinee, mais daus la morde lioss, m pleine zone antarelique, quela "bis-

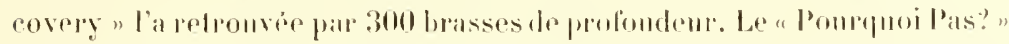

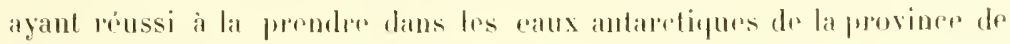
Magellan, on peut eonclure quel'espere est pour le moins partont répandue dans les oceians dusind.

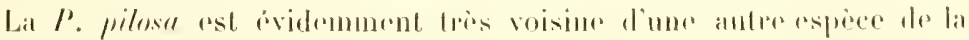

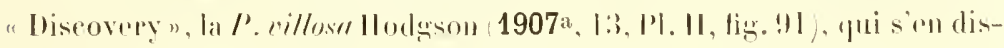

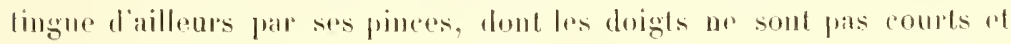

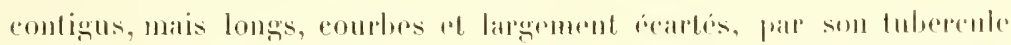

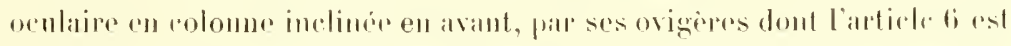

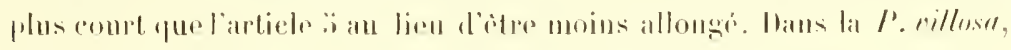
les demx articles du seape des eheliceres sont bien distinets.

\section{Pallenopsis glabra Mribius.}

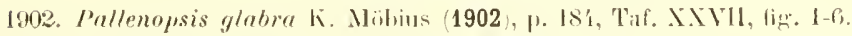

1007. Pallenopsis glabre T. V. 110drson 1907). F. 11 .

1911. Pallenopsis glabra E.-L. Bonvier $1911^{\mathrm{D}}$ ), J. 1139.

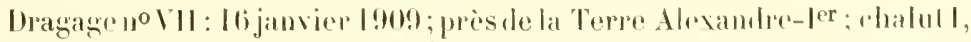
gö0 melres; lemperature de l'ean sur le fond + lo, fi: roche.

Ln exemplaire (

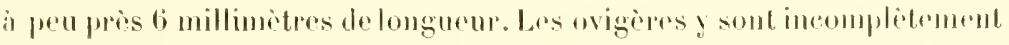

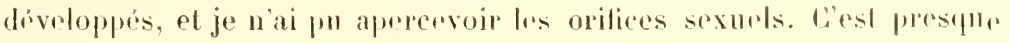
ecrlaimement un male.

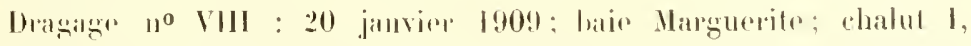
200 metres; temperature de l'ean sm. lo fond + 10. Is; roche, gravisr. vase.

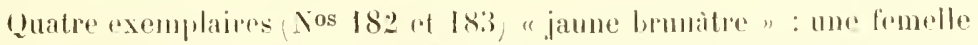
dont le ecpphalothorax mesure 12mm,

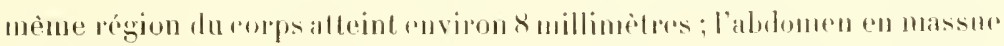

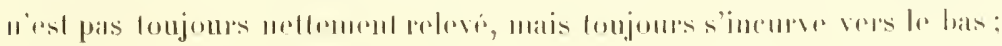

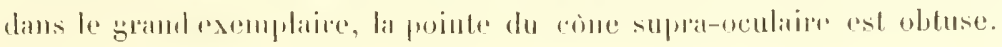




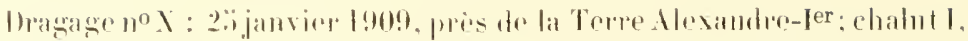

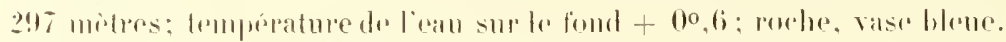

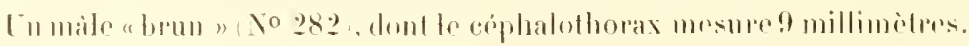

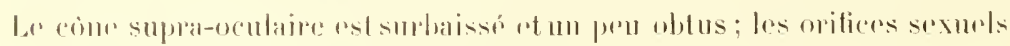

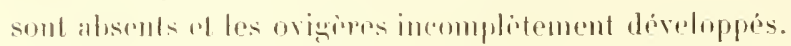

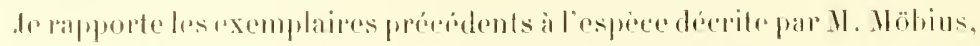

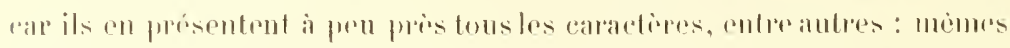

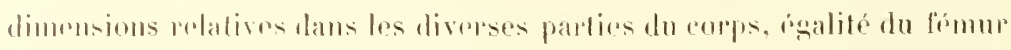

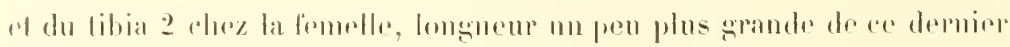
article dams le male, fome de l'abdomen qui ast en massure el recourbé

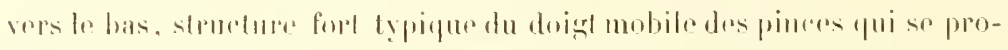

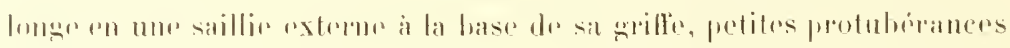
iparses sur los triguments motamment sur los pattes) ot qui sorvent de

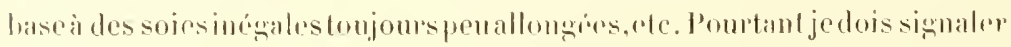

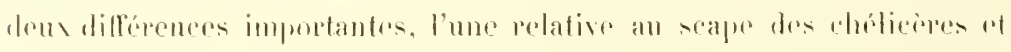

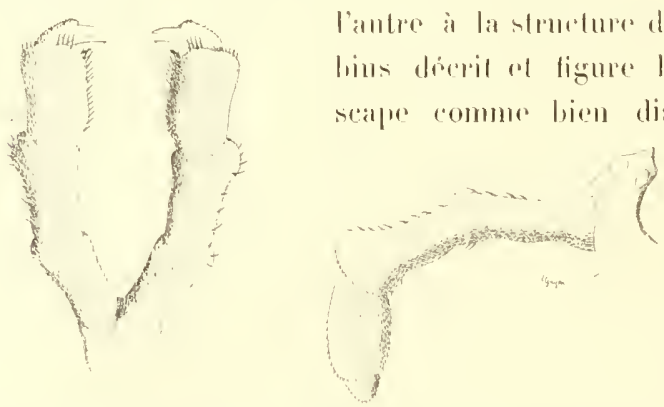

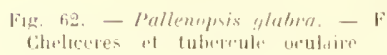
flun immalure, fate dorsils. iir. 6.

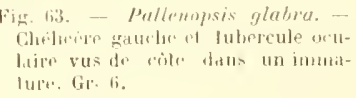

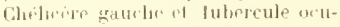

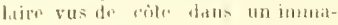
lure. Gr. 6.

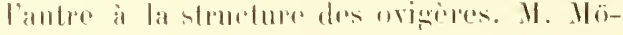
bes derux articles du seaper commo been distimets, aloms yo'ilo somt complive ment lusiomnes damslesesemplajpes pricrients, ail |o seil reste de lemp arliculalim primition est un emole lingingemint rimlle, lo

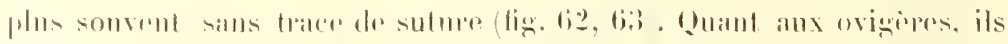

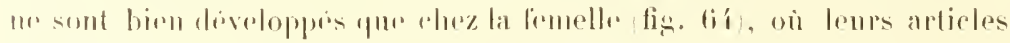

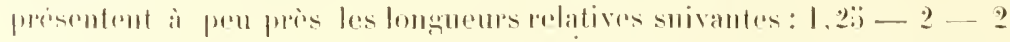

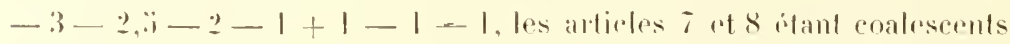

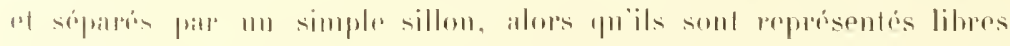

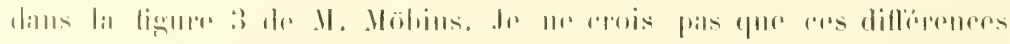

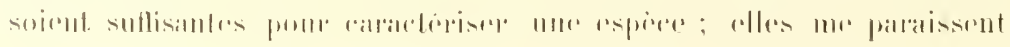




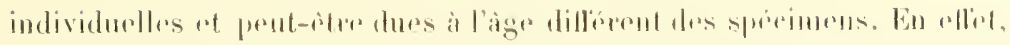

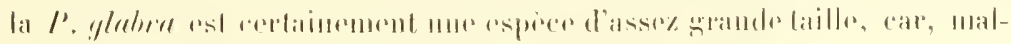
aré leur dimension resperelable, tous les exemplaires que je liens pour des mâles sont immatures, sans orilices sevmels ap-
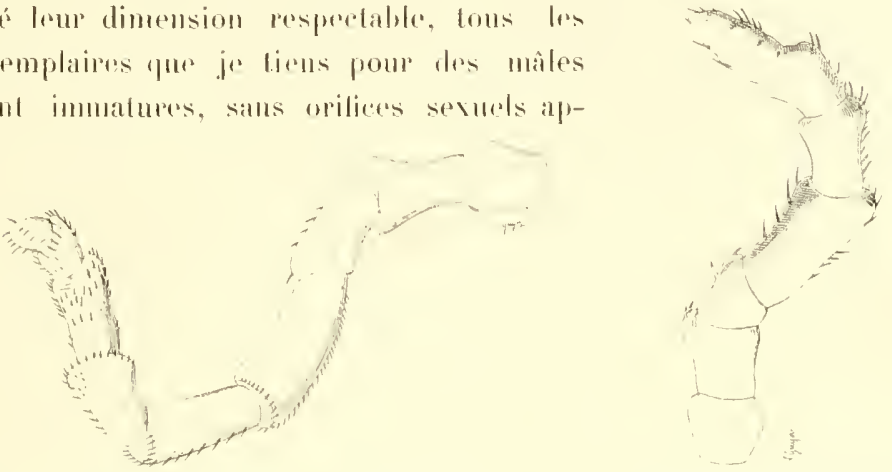

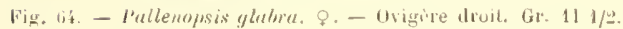

Fig. bis. - Pallenoprsis glabra. Ovigire d'un immalume. Gr. 15.

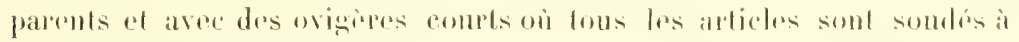

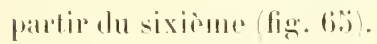

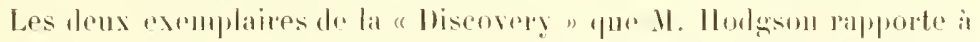

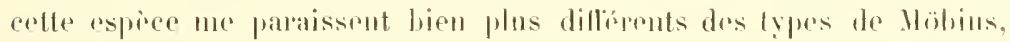

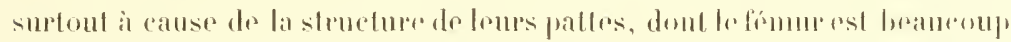

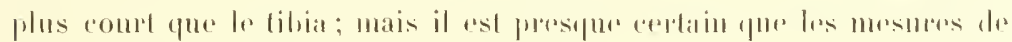
11. IJorlysont ont ido prises sur un male.

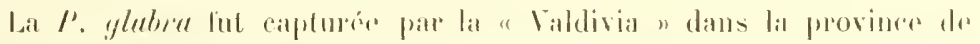

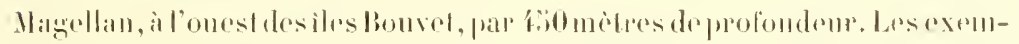

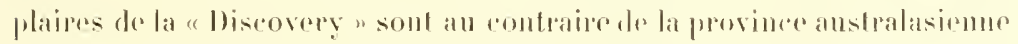

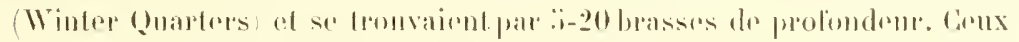

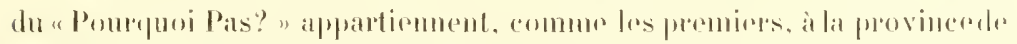

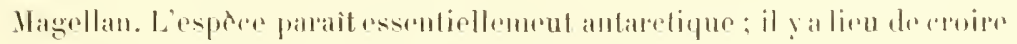

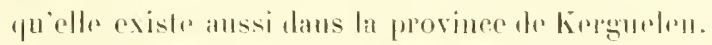

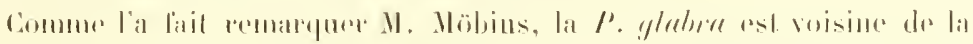

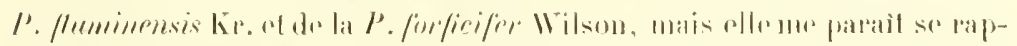

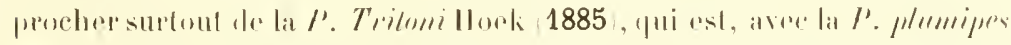

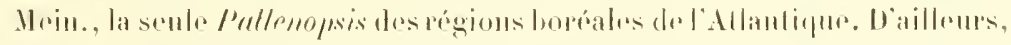

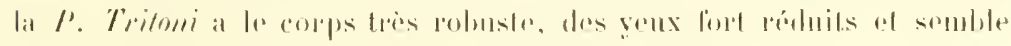

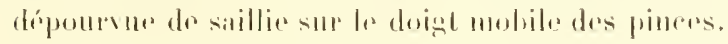


Pallenopsis macronyx E.-1. Bouvier.

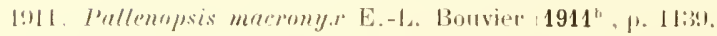

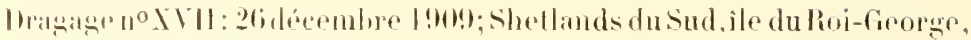

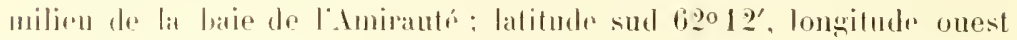

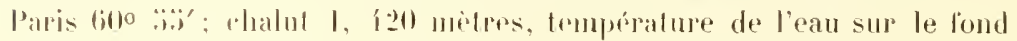
$+00,3$; vasr, raillous.

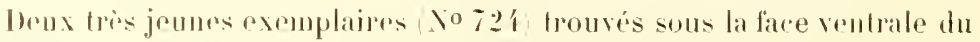
Vymplomestylogs (loir plus haut, p. $7: 3$.

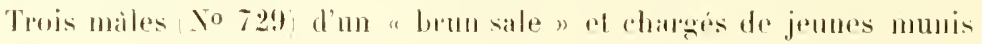
prestpure tous de leurs huit paltes.

Trejze exemplaires yo 730 , la plupart des femelles, fous d'un "brun jaunatre "): certajus males ave des ands. Lne vingtaine de jeunes, les uns queldue peu plus grands yor ceus portés par les malus du 10729. Jus aubres, plus nombreux, ayant déja des bourgons ovigères ret

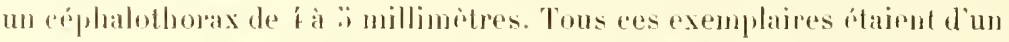

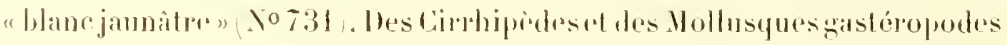
sont lixás sur beamoup d'exemplaims; en oulre un joune tromsé par 11. Kirbler sur un Lophaster nor. sp. ritipuetio ilo.

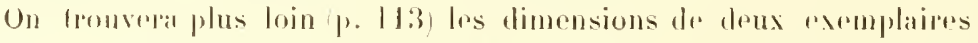

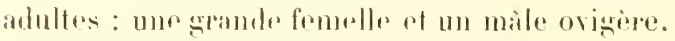

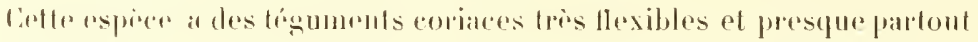
meouverts dinne inlinito de poils tries eourts, à peime perepelibles, qui rofienment facilement la vase. Elle a ro outre les soies caracteristipurs. yue muts itudieroms phus loim.

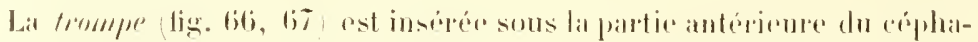

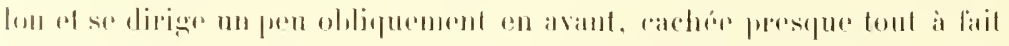

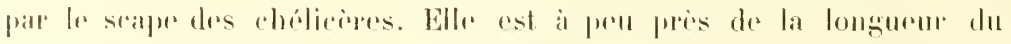

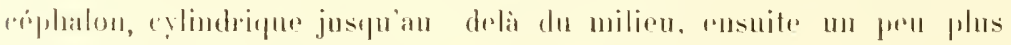
ilruitr: son hout rst arrondi.

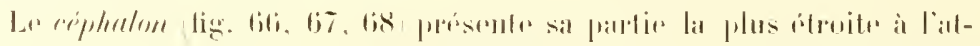

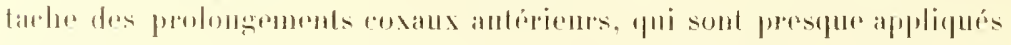

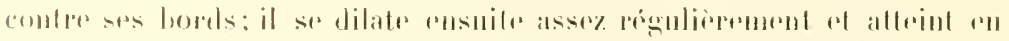

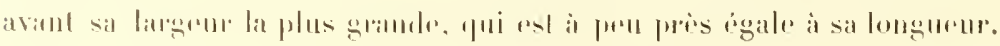




\begin{tabular}{|c|c|c|}
\hline & $0^{\circ}$ & $\dot{\circ}$ \\
\hline 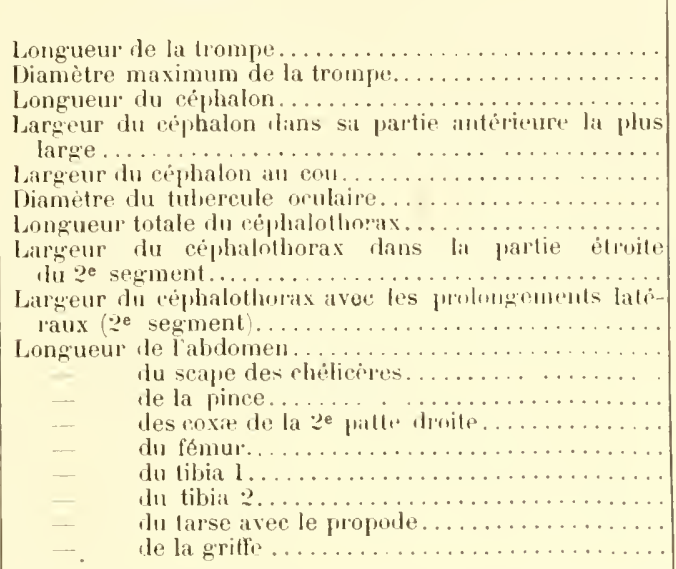 & 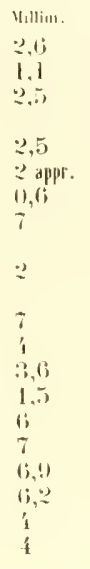 & $\begin{array}{l}\text { Hillin. } \\
3,2 \\
1,3 \\
2,7 \\
2,6 \\
1,7 \text { appr. } \\
11,7 \\
7 \\
1,8 \\
6 \\
1 \\
23,6 \\
1,5 \\
6 \\
7 \\
6,8 \\
6,8 \\
4,3 \\
1,3\end{array}$ \\
\hline
\end{tabular}

Convese du cótí dorsal, il porte un pen en arrière dr son lond antérient

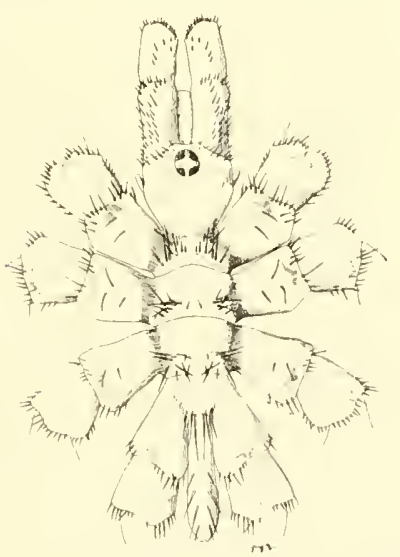

Fig. liti. - I'allenopsis macrony baur.. G. -

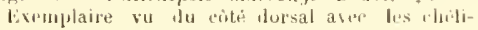
riros to la base des prittes. fir 4 1/2. arrondile tuberecule optique qui est

vertical, se dilate un poru dans la

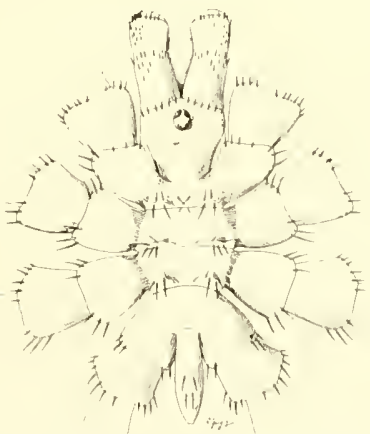

Fir. 6i. - P'allenopsis mocrony', Q, immature. Eximplaire vu du eith dorsal avec les chélicites et la hase di.s pallis. lir. $61 / 2$.

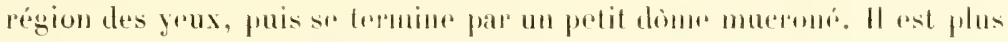

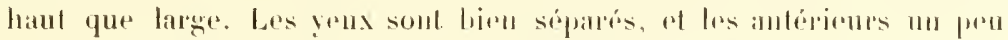

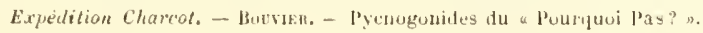




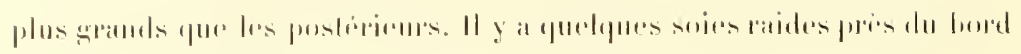
antíriour du réphalom.

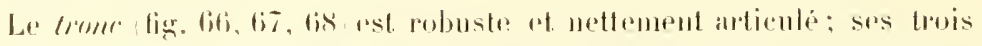

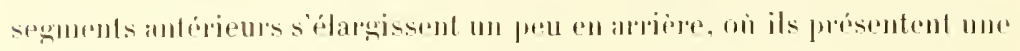

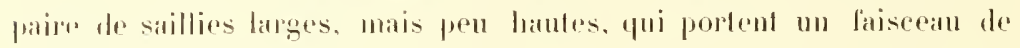

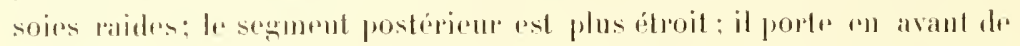
liabdomm deux grompers de deux ou trois soies. Les probmgements

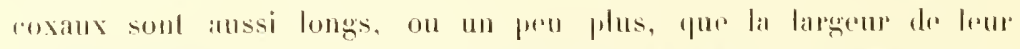

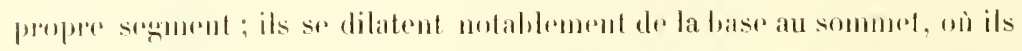

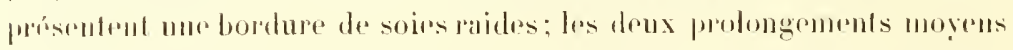

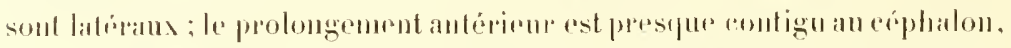

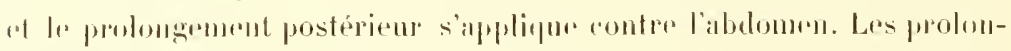

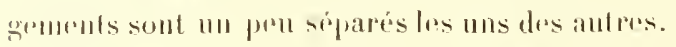

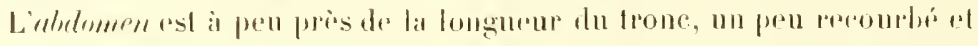

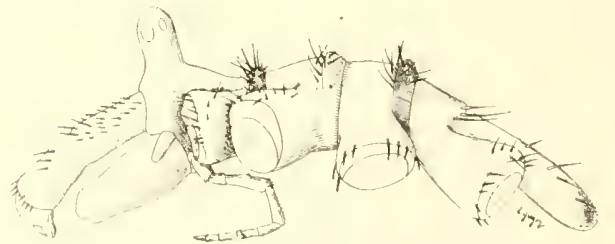

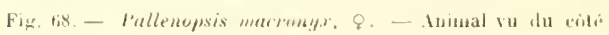

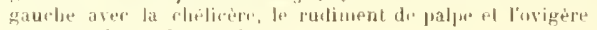
inerespulants. Gr. $51 / 2$. inthebi vers le bas; il se dilate regudierement jusqu“à la maissance de son tiers lerminal, "pris ynoi il se ratrécil un peu prour se torminor rol tom bust arrondi on laiblement rebanere: il est orne als quelpues sojes raides.

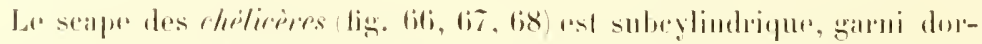

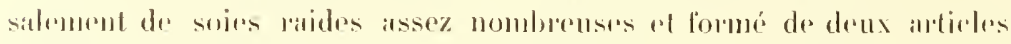

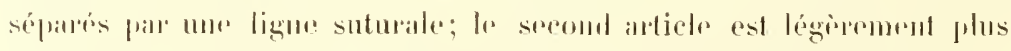

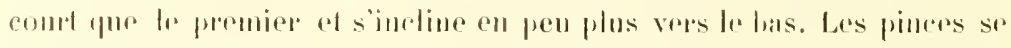

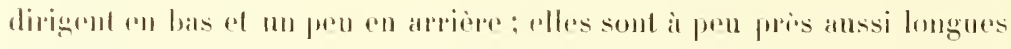

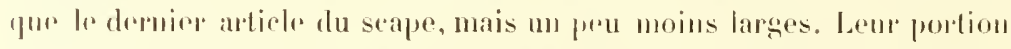

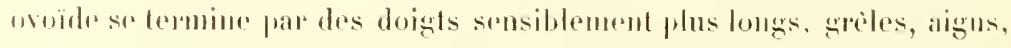

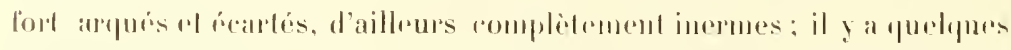

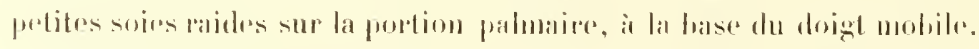

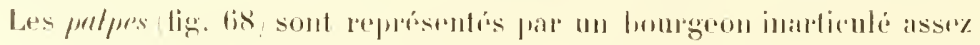
forl, mais phos lomg you lanker. 


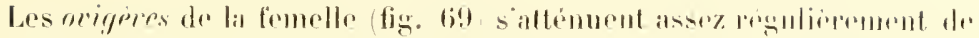
la base au sommet, les qualpe dernimps anticles eftant fort elpoils; la

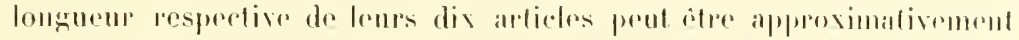
representé du premier an dixirme par les nombles : 2 - $1-3-6-$ 7-2 - 2 - $11 / 2-11 / 2 ;$ il ! i puelyurs menues soies mides sur le
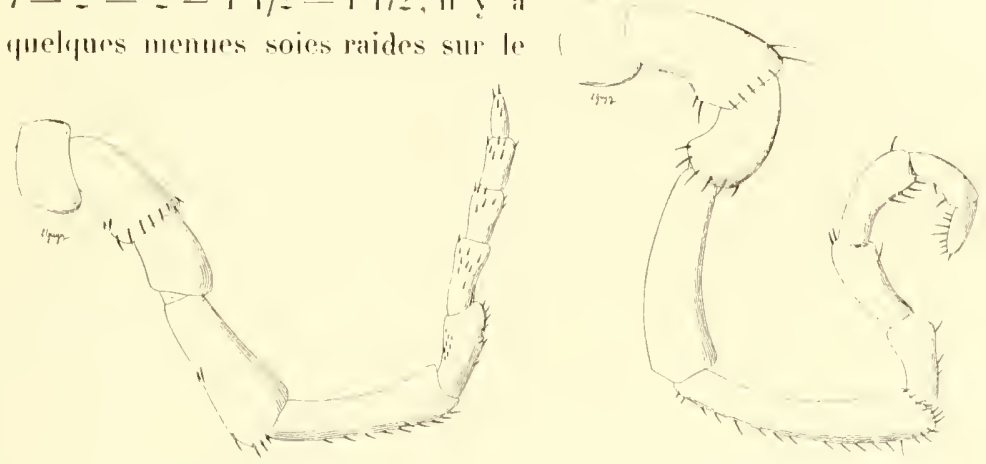

Fig. 69. - I'allemmsis mecronyx, ค. - Ovinim arathe, fir. 11 1/2.

Fig. 71. - Puldenopsis matrm!r, ot, - Wigne droit. Gis $111 / 2$.

depnier article. - Las ovigimes du màde fig. in sont assez dillirents, Wahord par los dimensions respectives de lenrs articles: 1 1/2- 1 $31 / 2-6-7-3-3-3-2-11 / 2$, emsuite prar la forme de ces derniers; les trois premiers articles se developpent en une rourber les denx suivants rn une rourlo opposen: les autres sont moins grèles que dans la fromelle: Io dixieme est lancéolé. muni d'une perite pointe, et, sur son hord interne, de quelques soies raides; il y aussi quelyues soies sur los articles prócridents.

Les proltes fig. il sont polativement couptes of fortes; Inurs articles coxaux se dilatent de la liase an sommet, le deuxieme étant un peu plus long que le troisieme et presque deux fois phus que fre premier. Des lrois articles snivants, fe fómur est leplus long el anssi le plus fort, surtont chez la femelle; Ir libia I est plus court, le tibia 2 l'esl davantage. Ce dernior articte est muni dorsalement de fortes sojes spiriformes pui ronsti-

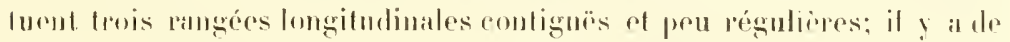

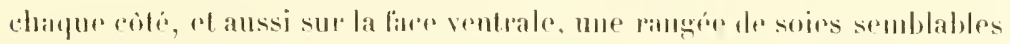

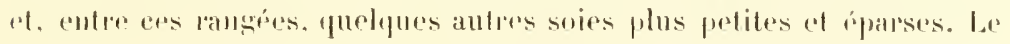


lilia I prosento me disposition semblable, mais la rangre ventrale est

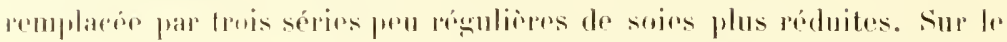

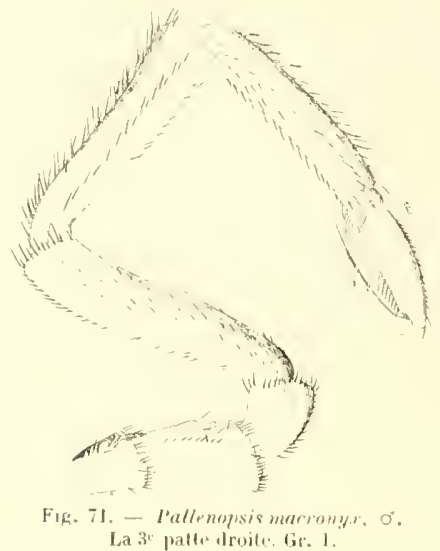

grompe dos mome epines sur la fare interne du tarse. La gritle ferminale rot anssi longue que les deus anticles procridents réunis, dou le

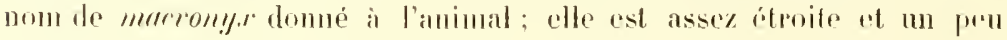
arefuces.

Les orifies sexuels du målr sont ponds ot silués sur mon légere saillie ventrabr, à une liable distaneo de l'extrémité distale de la rleuxieme coxa des paftes dres deux paires postérieures; unc saillin ot un orifice analogrues se trouvent vouladement au milieu du fémur de toutes les pattes figg. Il). Les formations lémorales n'ixistonl pas dans la femolle, qui présente d'aillours, commo lo màle, une légere protubérance sur le milieu du dos de la druxiene coxa de toutes les pattes. Les grands orifices suxuels de la fromelle se trourint à peu prìs à la monme place que ceux du male, mais sur loutes les pattes. Les ants onl un diamàtre de $600 \mu$.

Allachés anx ovignimes de corlatins màles, on trouve des jeunes à divers slades. Les plus avancés figg. 72 ant ume trompe prestue verticale,

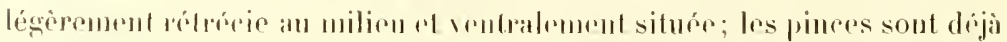

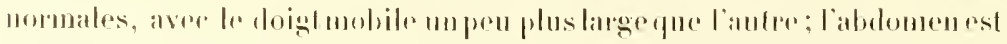

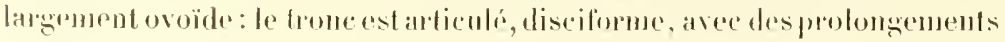




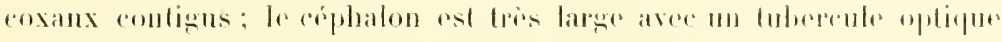

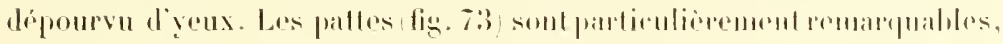
avere lomes cosir comples ol smbegales, lom fémul un

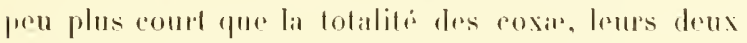

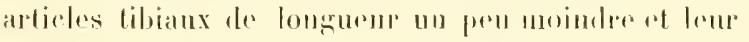

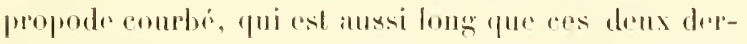
niers articles réunis: la wrifle. fres arroure est plus rourle que lo propole, sams grifles auxiliaires. Les

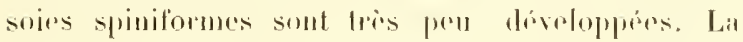

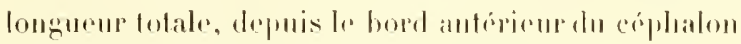

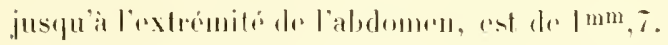

Les immalures lilues du vo $7: 31$ soml encoro dis-

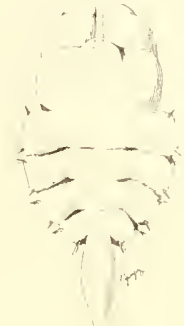

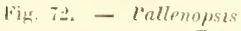
marronzy, - Tris jeun' cxemplaire aven sis ehition's. (ir) 93

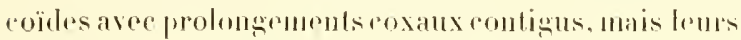
palles se mapprochenl davaulage de rolles de l'adulte ; rertaius sont

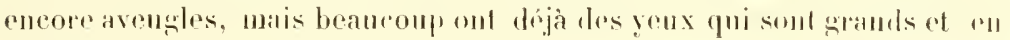
contact.

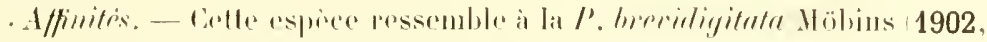

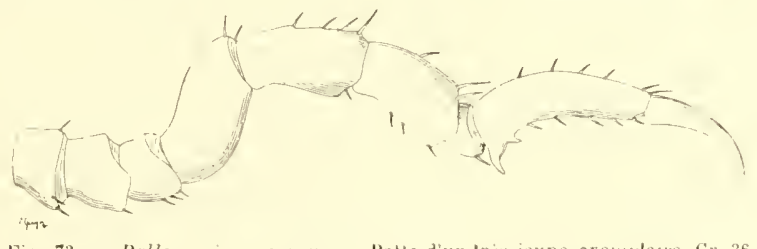

Fig. 73. - Pallenopsis marronys, - Palle d'un tris jeune exemplane. Gir. 36.

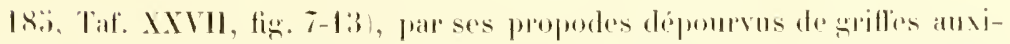
liairs et par ses griflins principales fres longurs: mais ollo en dilliom par tous les autres atacieres, motamment par som corps hien plus entdensé, ses pattes birn plus comblas, som large céphalothoras, et les soies spiniformes ou raides des diverses parties de son corps. Lat I'. hew vidigitata fut prise par la "Valdivia " dams la mer des ludes, flas

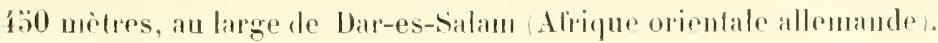

\section{Qualtieme famille. - PHOXICHILIDAE.}

Les Ploxichilidés font maturellement suite aux Phoxichilididés, dont 


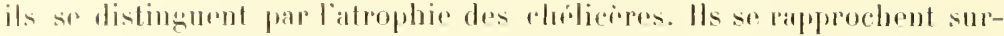

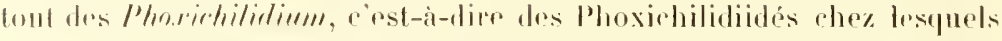

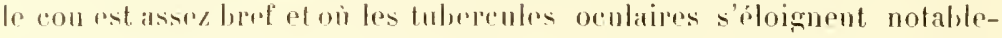
mout du bord frontal; mais s'ils sont. comme eux, dépourvos d'ovigipes choy la lemelle, les ovigores du male sont plus primitils et romptent 7 articles au lien de :̈; d'ailleurs, le cophalon est hion plus róduit.

Jans I0 systim, de M. Sirs (1891), que jai suivi partiellement dans mon budr sur les Pyenogonides du " Francais" 1906", les Phoxichilides sout disjoints des Phoxichilidiiles of remuis aux l'yenogonidrs dans te zroupe des Achélates. Cotte faute fut d'alord commise (1881 a, 3:i), mais

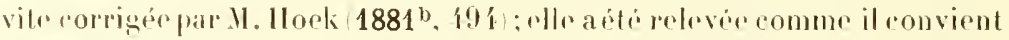
fall II. Carpenter 1894, l!18 ot par M. Loman 1908, 19 : si les Phoxirhilidés ressemblent aux Pỵogonidés par la disparition des rhólicòres. ils s'm distingurnt par toute lenr morpholosie, par la structure dilfopento

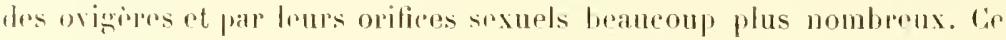
soml him des xymphonomorphes du fype des l'hoxichilidides.

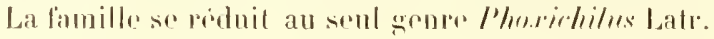

\section{Genre Phoxichilus Litr.}

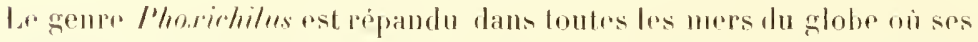
represembals habitont les zomes liltomale ret sublittorale. Il comprend

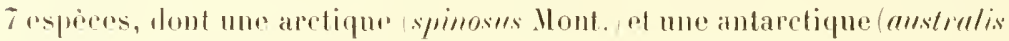

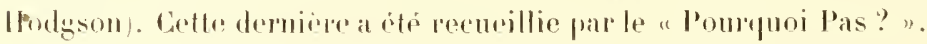

Phoxichilus australis T. I. Horlirson.

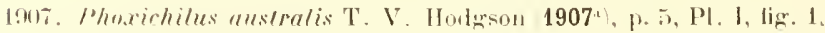

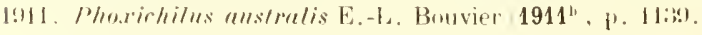

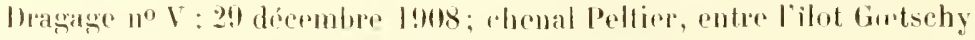
of lib lommer ; chalut 11,92 motres: temprimature de l'eau sur le fond - 110,1 ; rase grise, sraviru.

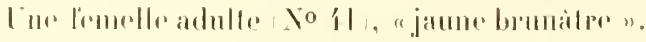

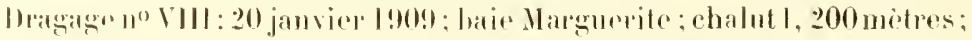

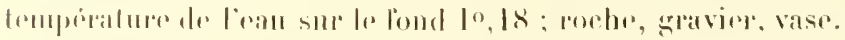

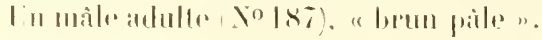




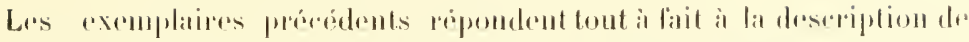

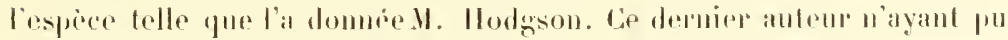

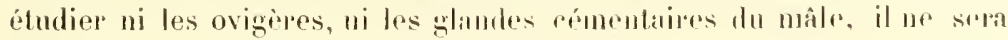
pas inutile de combler ceelle lacmur.

Les wigères fig. Th dessinent mo combe macieuse dans larpulle entrut leurs cing domiens ardicles; ils sattémurnl assez réguliopement pour ste terminer "n pointe un

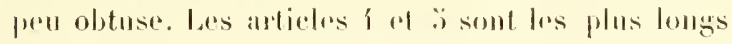

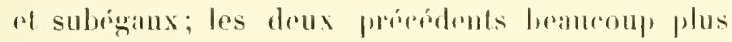
rompts el egalement subrgatux; l'article i se dilate dans sa partir terminale, oil il présente d'ailleurs un léger étranglement. I part yuelyues rares soirs courtes of raides situres sur le dremier article, les ovigires ne possielent pas d'autre revitement pileux que celui des pattrs, cest-it-dime de

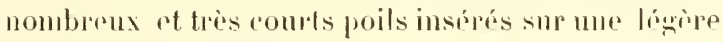
rugosifi trienumentaire.

Les oritiens des mandes à ément somt silues sur

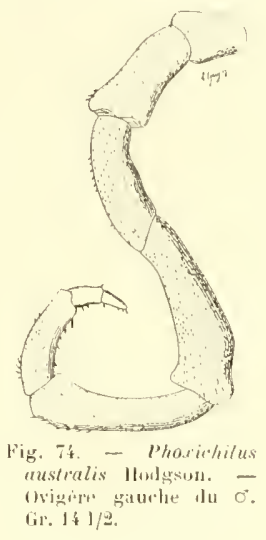
de petites saillies qui forment une rangér longritulinalo à la fance postririrure des pattes des trois drmiores paires. Ces saillies onf an plus un diamolere de 50 y, mais leurs dimensions variont notablement, do mòme

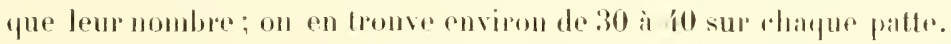

sur la face dorsale de ladeuxicme cosale toules les paties, on observe, dans les deux sexes, mepelitesillle obluse au sommet de lapuello vient

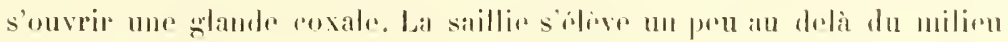
de l'irticlu.

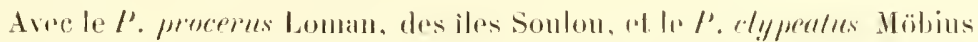

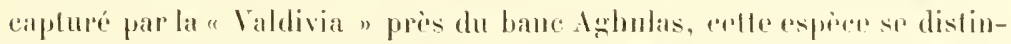

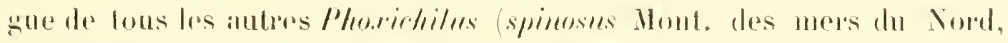

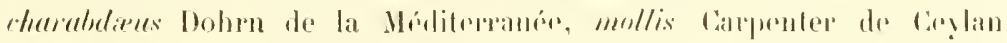

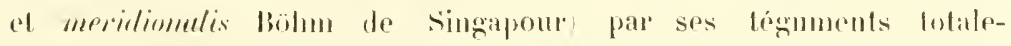

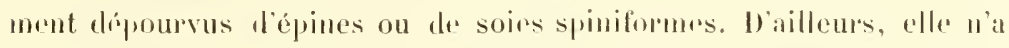
point lo corps condense ni les paltes fortes du l'. clypentes, mais alle

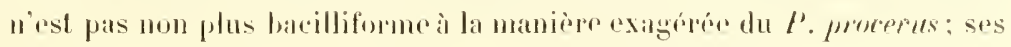




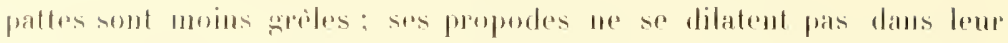

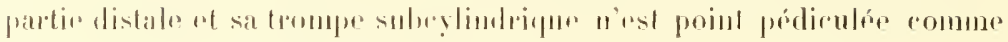

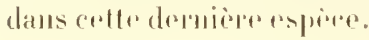

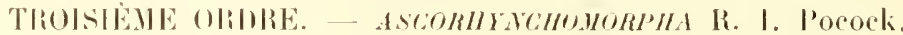

liet omdro correspond presque exactement aux Cryptochálates de M. Sars

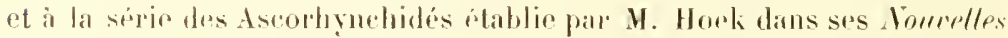

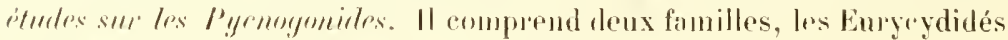
et les Ammotheidés, pue 1l. Loman (1908) sipare tomt à fait ot place dans dens serpes distinetes en se fondant sur la structure des ovigères. J'ai domé phus haut ip. Á les raisons qui mont porté à établir le groupe

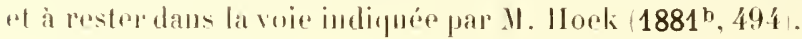

\section{Premiere fimille - EURYCYDIDA.}

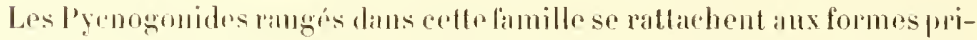

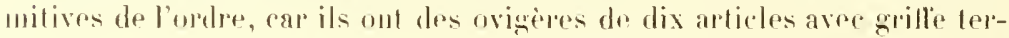

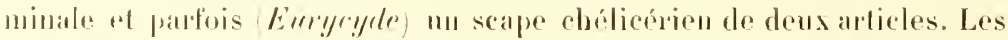

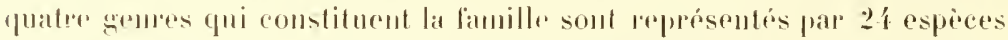
pour la plupart sublithrales, dont i aretiques of l antaretique. Cefte

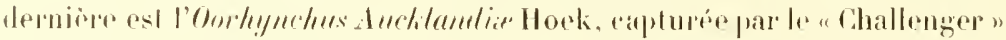

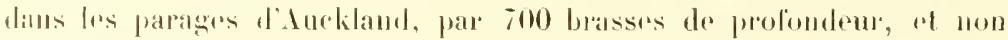
retromiendepuis.

\section{l)euxieme famille. - AMMOTHEIDAE.}

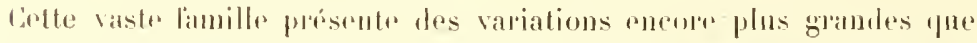

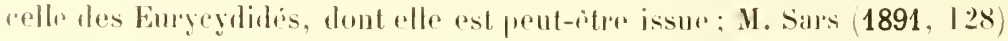

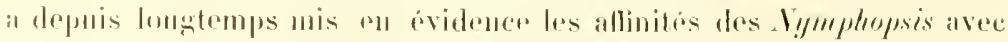

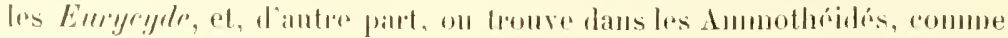
dans los Euryeylidris, un cortain nombre de lommes à palpes tris réduits

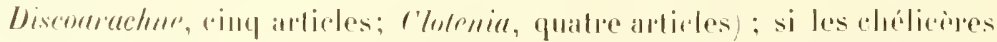

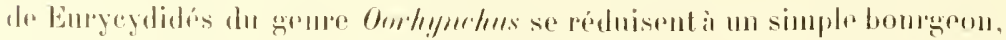

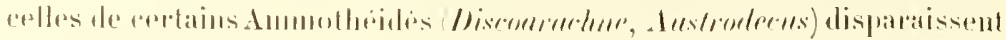
sitls ilueull, llater. 
Il me parait bien difticile, dans l'état actuel de nos connaissances, de relier entre eux les divers genres de la famille; a l'exemple de MI. Loman 1908), je les répartis en deux sous-familles : les Nymphopsinés el les Ammothéinés, dont les caracteres ont été relevés plus haut (p. 1:-2). Avec leurs chélicères dont le scape a fréquemment denx arlicles ef avec leurs palpes de neuf articles, les Nymphopsinés se rapprochent beaucoup des formes primitives de l'ordre Euryryde), mais les Ammothémés dn grenre Bhynehothorar el les Ilamonia s'on rapprochent aussi par leurs ovigères, qui sont, par exception uniqque, armós d'une grille terminale. II ne saurait d'aillenrs êtroquestion de faire dériver les Ammothéinés des Rhymohothorax, mais ce genre nous montre que la sous-famille provient, elle aussi, de formes primitives, voisines des Euryeydidés on des Eurycydidés euxmèmes.

La sous-famille des Vymphopsinés n'est pas connue en dehors du Pacifique, oì ses représentants habitent les eaux clıandes sublittorales; les :̈ genres qui la constituent renferment en tout 10 espèces.

La sous-lamille des Ammothéines ast beancoup plus riche, ear alle

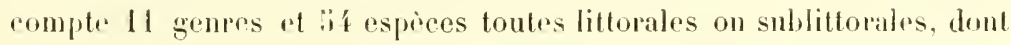
7 aretiques et $\mathrm{I} 8$ antaretiques; ces dernieres se répartissent entre les

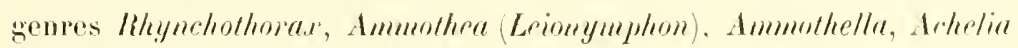

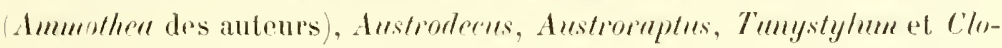
temire. Les caractères essentiels et la place de ces genes sont indiqués dans le talleau suivant, gui s'applinge ì toute la sous-famille: 
Les Ammothéinés conmus dans les mers antaretiques appartiennent anx genres Rhynchothorar (2 espèces dont une antarelique), Ammothea In esprices toutes antaretiques, sauf une seule), Ammothella (6 espices.

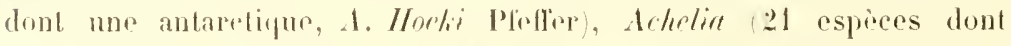
li aretiques et 3 antaretiques), fustromplus. (1 espece qui est antarelifjur), Trmystylum 7 especes dont 3 antaretiques, parmi lesquelles le

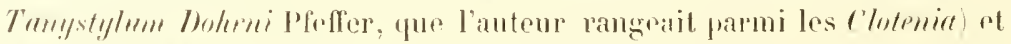
Austrouteces l asprice qui mit antaretique.

Les espires peremillies par to "Pourquoi Pas? " appartiennent aux

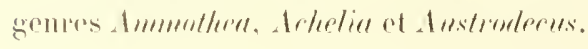


Genre Ammothea Learh (Lrionympleon Nibius).

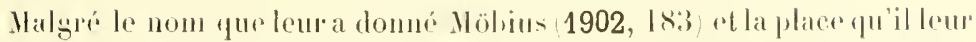
attribuail dans la famille des Nymphonidis, les espeees du gente dmmothea sont des Ammothéides sans conteste, ainsi que l'a reconnu Il. Ilodgson, le savant historiographe de ces curienx Pyenogonides (1907a,39). J'ai exposé antérieurement $\left(1906^{\text {b }}\right.$, : i i les laisons qui justifient ce classement; il ne me reste aujourd'hui yu'i relever, en le moditiant un peu el en li complétanl, le tablean où M. Ilodgson a mis en relief les caractères assentiels des diverses espeeces antarctiques du genre:

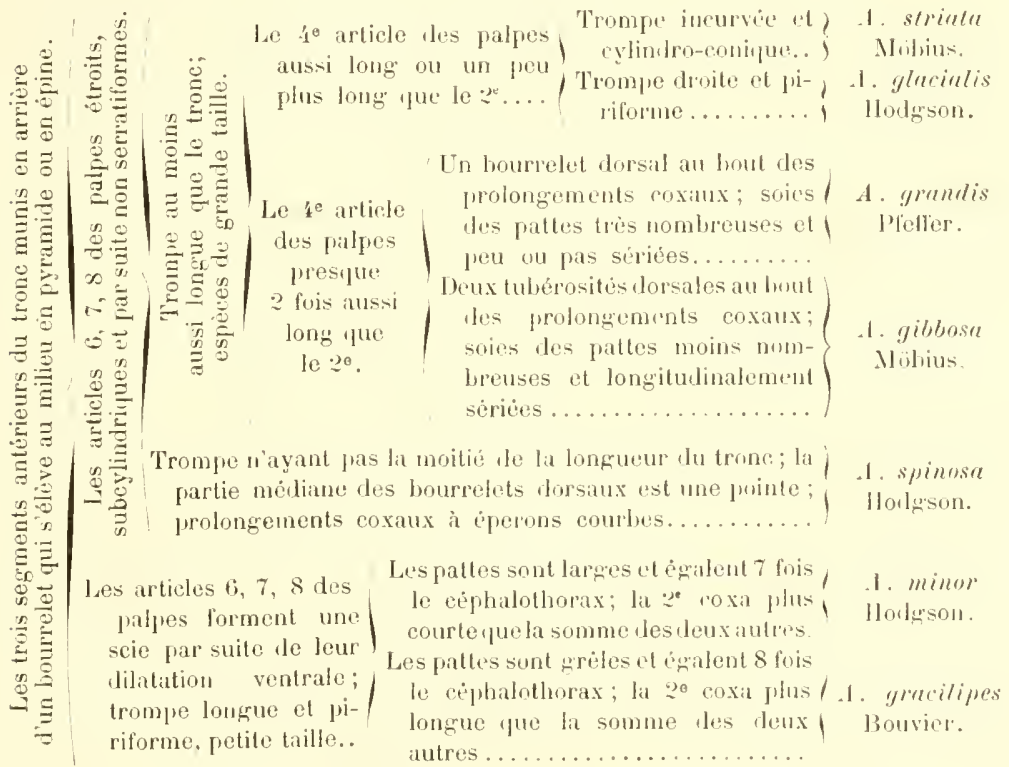

Un bourrelet bas et sans / Ablomen subvertical, atteint par le lord, 1. rifausi

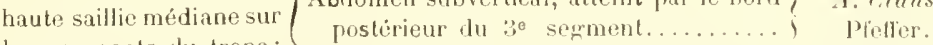
les segments du trone; Abrlomen oblique éloigní du bord pos- ) A australis

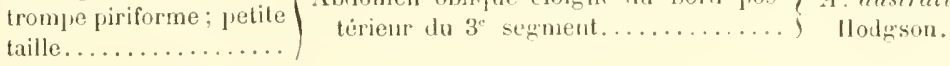

En dehors de ees especes, le genpe Ammothen n'en comprend qu'une seule, l'd. corolimensis, qui habite la Caroline du Sul aux Etal-Luis el pour 
lacuello fut śtahlie par Leach, en 181 f́, le genre Ammothea. On a vu plus haut (p. fii) comment II. Loman et Mt. Hodgson, à des titres divers, ont contribur à itablir l'identification de cette espèce avec les Lriomymphon de Möbius, ce qui a conduit à remplacer ce dernier nom par celui de Leach. L'A. cruolinensis est la seube espèce non antarctipue du genre; par sa taille, lia structure do ses palpes, la forme de sa trompe, la saillie de ses bourrelets dorsaux, elle prend place à coté des .1. grandis et gilliosa.

\section{Ammothea striata Mobius.}

1912. Leionymphon striatum K. Möbius (1902, p. 183, Taf. XXVI, fig. 6-12. 1!11. Leionymphon striatum E.-L. Bouvier (1914 ), P. 114 4l.

Dragage no XVIII : 27 janvirer 1909, shetlands du Sud, anse ourst de la bair de l'Amirauté, dans l'ile du loi-George; chalut I, 7ä mètres; temprérature de l'eau sur le fond $+0^{\circ}, 2$; vase grise, cailloux.

Quatre beaux exemplaires (No T 10 ), femelles adultes d'un "brun sale" dont le céphalohhorax mesure environ 12 millimètres de longueur.

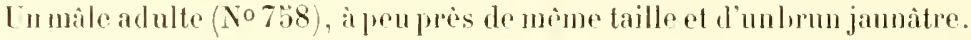

Ces exemplaires répondent tout à fait à la description ct aux figures donnén par M. Möbius, les senles dillépences provenant de légéres variations individuelles. Ainsi larlicle 3 des palpesest souvent prespue écal a l'article z, et le dernier un peu phus long que les articles qui te précèdent: l'abdomen est plutiot ovoüde que cylindrique, avee un sommet litracuent obtus.

Les caractipes les plus fraphants de l'esprece sont les suivants: Io réphaton tig. Tï n'est pascétrécen arriere, mais s yéprime latiralement comme pour indiquer le con; il ast oceupé en son centre par le lubereule oculaire, yui est un large trone decône vertical lerminé par un còne bas ; les saillies postéro-dorsales médianes drstrois segmentsantérieurs du trone sont léxèrenent inclinées en arrière, et largementobtuses an sommet, ‘ui porte de nombreuses spimbles: - les prolongements coxam prísentent à leur extrémité deux larges protubérances dorsales contignïs et peu élecées, qui portent des spimules semblables aux précédentes, mais plus réduites; lit longuenr des prolongements est telle que la largeur 


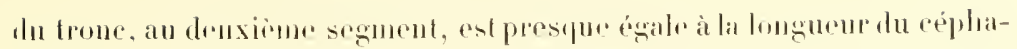
lothoras; - lahdomen est anssi lomg pue le cephalon et s inllechit vers le

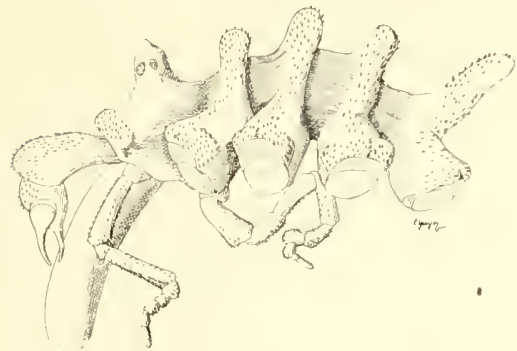

Fig. 73. - Immothea striatu Möbius, o. - Le corps iu du cotté gaurlas, ave les appendices anticicurs correspondiuls. (it: $13 / \mathrm{k}$.

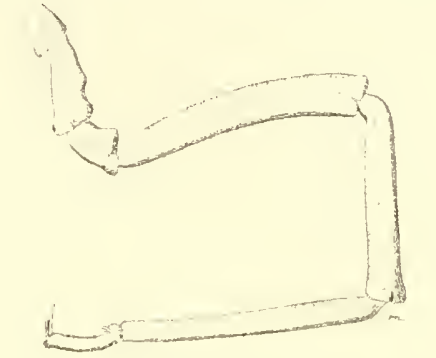

Fig. 76. - Immothen striati, $0^{\circ}-$ La 30 patte droite. Gis. I 3/4.

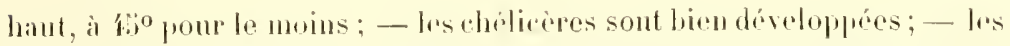
articles za f despalpes sont subégaux, de mômo queles cint suivants, qui ne se dilatent pas notalilement i leur angle antéro-inférieur: - le limur

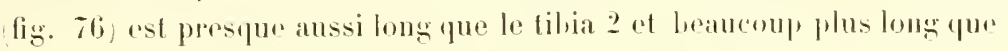
le tibia I; la strillo égale au plus la moitio du propode, qui est trios pen arque at sarrs fortes soies spiniformes sur son bord interne; les grill's anxiliaires qui laceompasment ágalont an plus sa moitié, onfin les spiumles despattes so groupent en siriesnellement sciparies par des espaces nus, les series latérales se composant prespue d'unesculerangeredespinules. J'ajoule yur la trompe est légerement phus

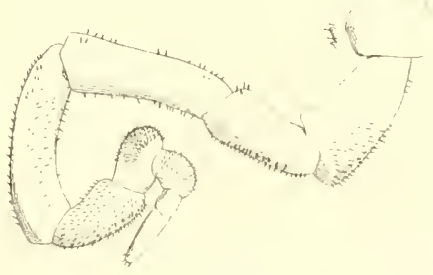

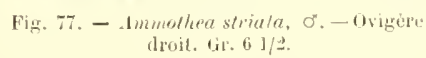

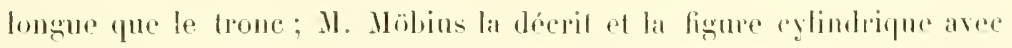

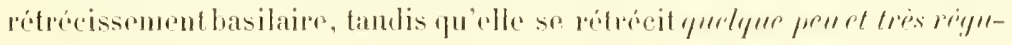
livermerent de la base all sommet (sur sa face superieure) daus fous lest

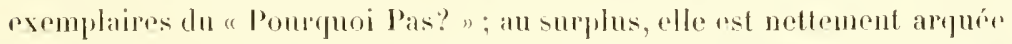
a infléchie vers le bas, comme dans les types de M. Möbius, de soltr que jatribue les diflérenees précolnutes à nn examen superficicl ot à une laute du dessinateur de la " Valdivian. 


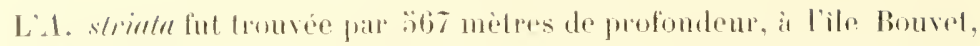
cers-it-dire dans la province africaime subantaretiepur ; les découvertes du "l'ourphoi l'as"? montrent que cest ingalement une espece de la province magellanigur ot des eamx antaretiques.

\section{Ammothea grandis ['feller.}

1889. Ammothen grandis G. Pfeffer 1889/, I). 13.

19h5. Colossenteis ?? Chareoti E.-L. Butuvier 1905), P. 2:10

190hi. Colossenteis? Chareoti E.-L. Bourier 1906" ,". 19.

1941. Leionymphon grande T. V. Hodgson 1907 . Y. Al, PI. VI, tig. 1.

1917. Leionymphon grande E.-L. Bouvier 1906'), p. 60, Pl. III, fig. (i et fig. 30-1́t du texte.

1918. Leionymphon grande T. V. Hodgson 1908), p. 179.

1911. Leimmyphon grande E.-L. Bouvier 1914\%, p. Il't).

Dragage ${ }^{\circ} Y: 29$ décembre lons, chenal Peltier, entre l'ilot fortschy et l'ile Doumer; chalut 1; 92 mêtres, lempérature de l'ean sur lo fond - 00,1 ; vase arise, gravier.

Un màle " jaunc orangé " ( †o 37 ), oủes pinees sont encore parfaitement normales, encore que le céphalohorax mesure déjà II millimitres; cel exemplaire nest sans doutr pas encorr adulte, car je n’ai pu y voir los orifices sexuels, of les ovigeres nout pas tout à fait lum lorme définitive. L'exemplaire est d'ailleurs un peu anorual.

L'L grandis est voisine de la précédente, mais elle s'rn distingue par sa taille plus grande, sa trompe droite of rétrécie a la base, son tubereule oculaire plus broil, ses sallies beancoup plus hautes ef légerement infléchirs an avant; son abdomen courl, à peine oblique, ast précédé d'un tubereule en cone : ses pahpes sont remarquables par leur gracilité ef par la

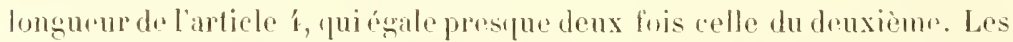
pattes ne sont pas moins caractóristiqurs : lemtibia 2 est tros notablement plus long que le fémur ; leme propode armé de soies spiniformes est plus recombé que celui de l'il. striata; les grifles principales sont pour lo moins aussi longues qur ce dernier article. ef les griffes anxiliaires en igatent au nuins la moitié; les téguments prósentent un asport chagriné, grace à des spinules nombreuses, qui, sur les palles, ont parfois une leggere tendance à la scriation longitudinale.

Cefte belle espece comple parmi les grands Pyenogonides; son corps 


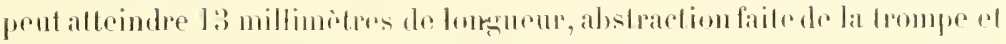

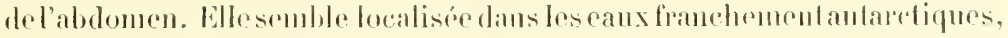

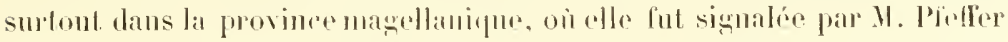
Grorgio du sud, 1: brasses), et relromvée par la "Seotia " (1)reades du Sud, l íbrasses), puis par les nalmalistes du "Frangais " (Port-lidareol, 20-2:i motres; île Booth-Wandel, 30 motres); on a vu plus haul quelle

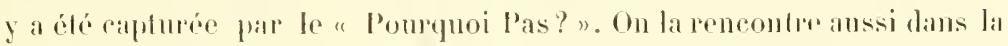

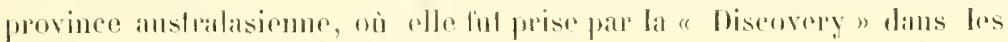
parages de l'ile Comluman ( 8 i l li lumsers).

La coloration sur lo vivant varie du jaune-soufre on orangé an rouger lipun.

\section{Ammothea gibbosa Mibius.}

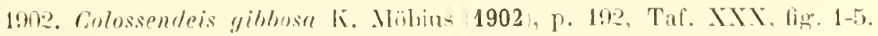

1906. Ammothen currulio E.-L. Bonvier $\left(1906^{4}\right)$, p. 201.

1906. Ammothera cureulio E.-L. Bonvier $\left(1906^{\mathrm{b}}\right)$, P. 4t), tig. 19)-2?.

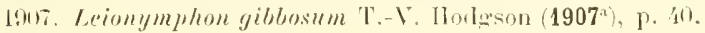

1911. Leionymphon gibbosum E.-1. Bouvier (1911"), p. 11'to.

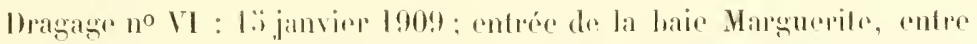

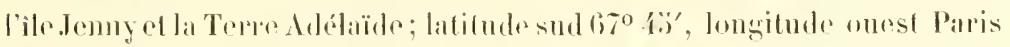

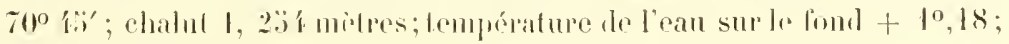
lorlese. wavior.

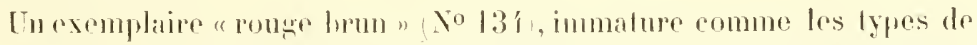

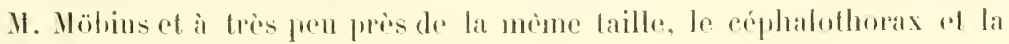

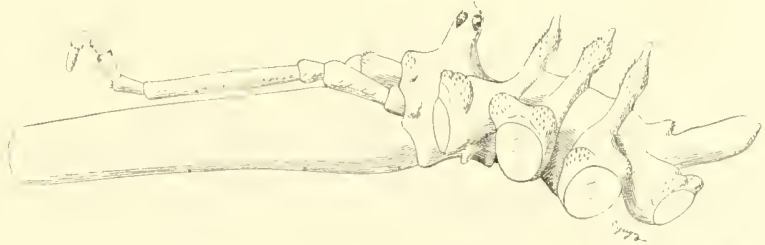

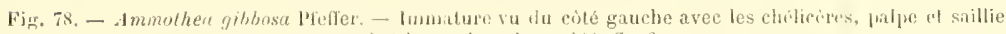
ovigérienne du urène còté. Gr. 8.

trompe mesurant chacun pros de 6 millimetres; ovigioes roduits à de

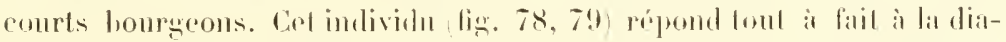

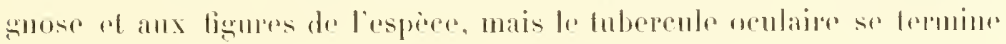




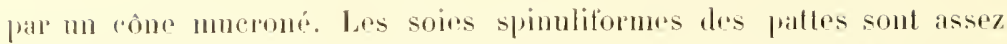
nombrenses of disposces en séries, presque comme dans l'A. strinlu.

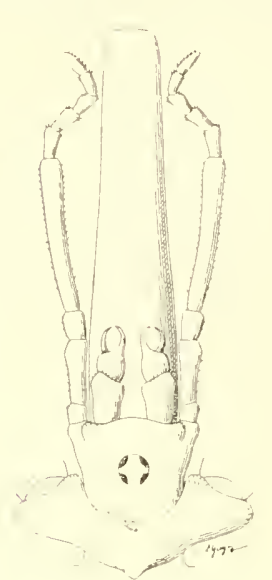

Fig. - $9 . \quad$ - Immolhea gibtosa. - Partie anlérieure du corps et ses appendices dans un intmalure, face dorsale. Gr.s.
La tromper ressemble à celle de eette dermiere espreen, mais ello est fort pen argmén el présente la vague indieation d'un etranglement ì puelque distance de sa base.

Dragage no voll : 20 janvier 1909. haie Marguerite; chalut 1, 200 modes ; lemperature de l'eau sur lo fond $+10,18$; roche, wravier vase.

In exmplatre " jaune lirant sur l'orangé " So 186, immature eomme le précedent, mais do taille un peu phos réduile; la structure est d'ailleurs la mime, tontefois le tubereule oculaire est arrondi au sommet ave mu léger mucron, If fómur n'rigale pas tout à fait le tibia l. el les spinules des pattes sont moins nombreuses el plus distinetement sériées.

Dragage $1^{\circ}$ VII: If janvier 1909, press de la Torme Alexandre; latilude

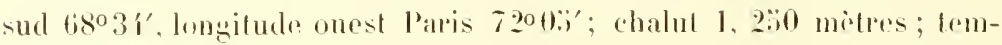
pérature de l'riu sur le fond $+1{ }^{\circ}, 6$; roche.

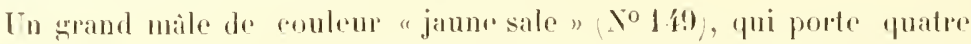

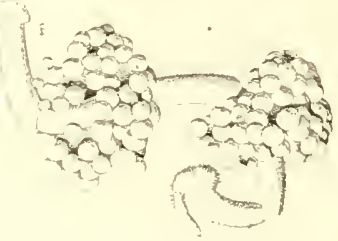

Figs. 80. - Ammother yiblosa, $0^{\circ}$. parpuets d'oufs fig. 80 en lorme de manchon, denx sur chapue ovigipe: l'un de ces manchons entoure l'article 4 , l'autre la partie distale de eet article et la partie basale du suivant: les arufs sont tres nombrens of mestrent a peu pres I millimetre de diat metre.

Cot remplaire fig. 81) differe des deux prédentset destypes immaturn's de N. Möbrus par sa trompe, qui ressemble à celle de li. I. grame à peu près droite, rétrécie et avec un leger fitranglement dans son tiers basilaire, dialleurs bien plus large dans ses deux tiers ferminaus, par ses petites chéliceres on la pinere 
est reduite à un bourgeon, par son tubercule oplique on colomm dilatéc et arrondie au sommet, pirr ses saillies dorsales sengmentaires qui s'épaississent beaucoup en haut, leur petito pointe peu appa-

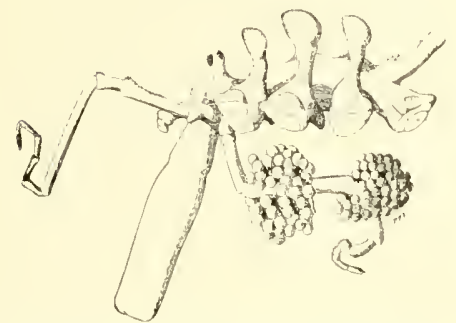

Fig. 81. - .1 mmothen gibbosa, б", - Le enples vu du còté gauche avec les aprentices céphalijues correspondants. Gir. $13 / \hat{k}$.

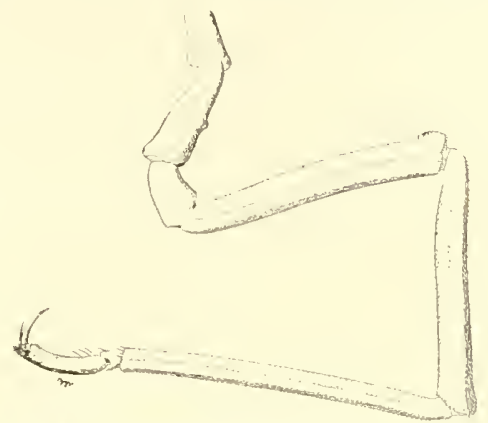

Fig. 82. - Ammothea gibhosa, O. - La $3^{\circ}$ palte fauche avec l'orifice sexuel ut la saillie coxale. Gr. $13 / i$.

rente etant situce en avant, of par les fémurs (ligs. 82) qui sont trís ligisrement plus longs que los libias 1.

L'exemplaire ollee d'elranges ressemblances avee l'A. grmmlis, mais il s'en distingue of ressemble aux tyes immalures de l'especer par quelques caractires importants: $1^{0}$ la plus grande hauteur des saillies dorsales segmentaires; $2^{\circ}$ la plus srande longueur ot la direction tros oblique de l'abdomen; $3^{\circ}$ le développenent de denx lubérosites arrondies à l'extrémité distale des prolongements coxaux et du premier article coxal, tubérosité qui remplace mo ligge bourpelet continu dans l'A. gramlis; fo les spinules des pattesqui sontboaucoupmoins nombreuses et régulièrementsériées (notamment sur lestihias), an lieu d'alrodisposées sans ordre. Pour le reste, les deux espieces me paraissent alisolument identiques, et jen suis encore à me demander, comme jo la laisais (1) 1907 (p.64), si elles ne sont pas identigues el ne devont pas ifro rémies dans la suite (1).

Je crois utile de relever ci-dessons les dimensions du mile adulte prócédent et d'un mâle ovigère de l’ A. grantix.

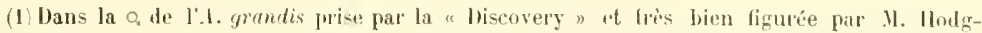
son 1908, l'l. Il, fig. I, les spinules du tibia sont neltement sérices, l'abtomen eal obliqum, ol la Lrompe cylindrique semble rétrécie à la base. 


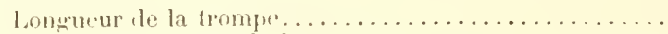

biametre maximum de la frompe................

le la trompre is Jextrimile distate. . . . . . . .

Longuent du céphalon. . . . . . . . . . . . . . .

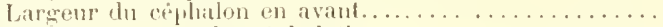

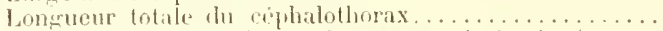

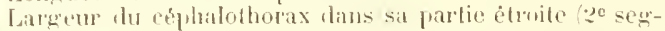

ment) ... . . . . . . . . . . . . . . . . . .

Largeur du ciphalothorix avee les polomentents coxanx

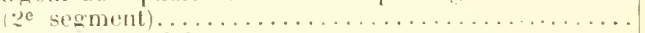

Longueur de l'ahdomen . . . . . . . . . . . . .

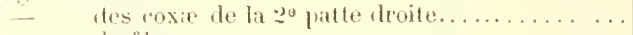

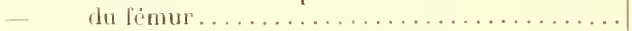

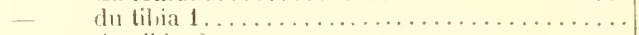

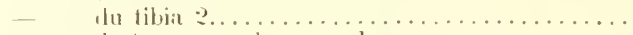

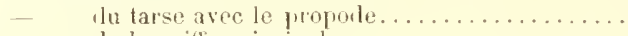

- de la sritle pincipiale.

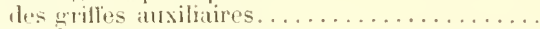

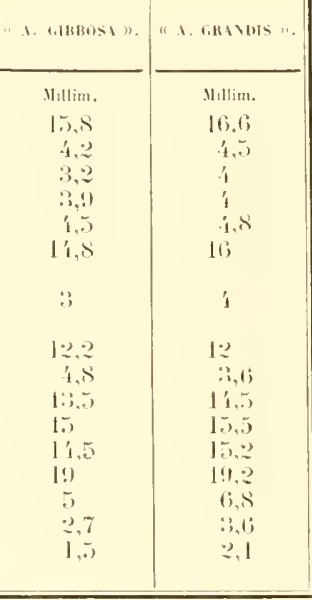

On voil par eo labluan pue les adultes des deus especes dillirent non

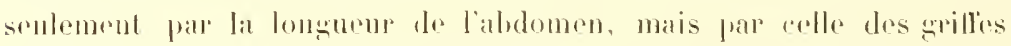

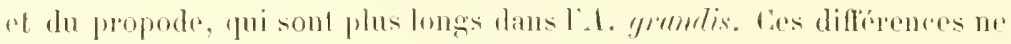

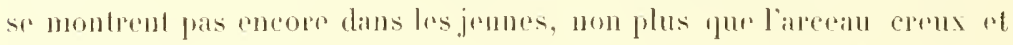
lisse pui se trouve on avint sur los protubrimaners dorsales, au-dessous dela dilatalion qui trmmine ers saillies.

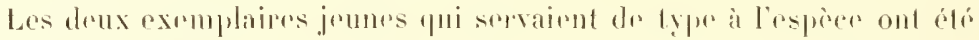

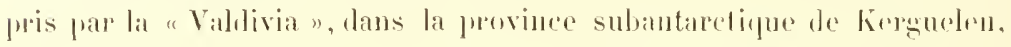

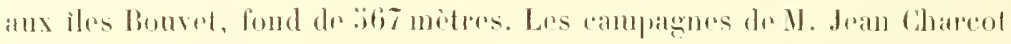

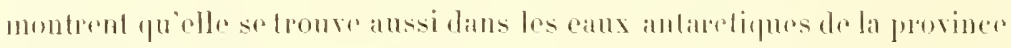

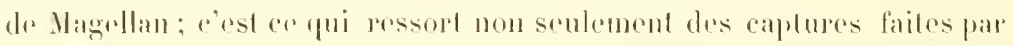

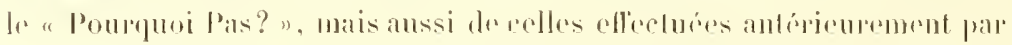

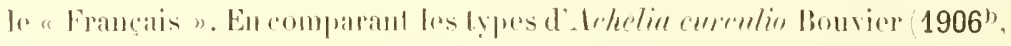

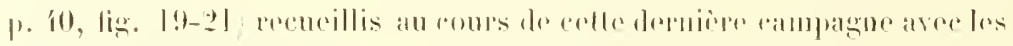

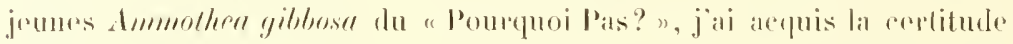

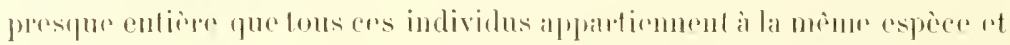

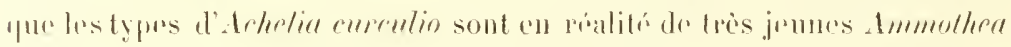
gibbere, beancoup plus jeunes et plus petits quoreux du "Pourpuoi Pas?".

En loul ads, l'espece subil avoe l'age drs modificalions profondes:

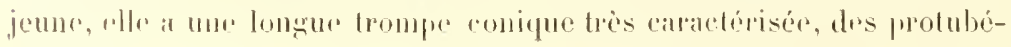




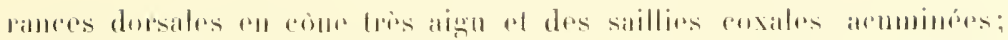

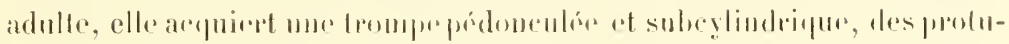

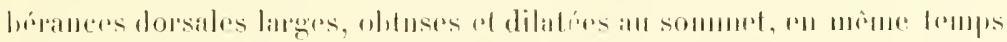

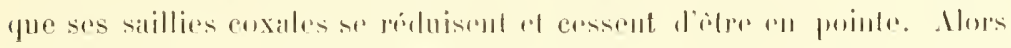

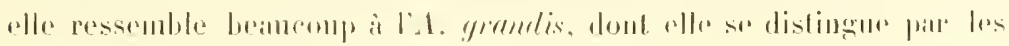
raracteres indipués phus baul.

\section{Ammothea minor T. I. Iorlgion.}

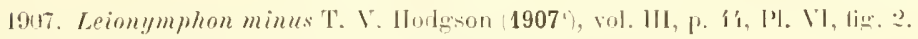

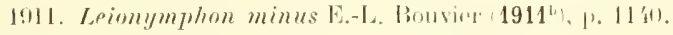

1911. Leionymphon minus E.-L. Bouvier 1911'), 1'. il'i.

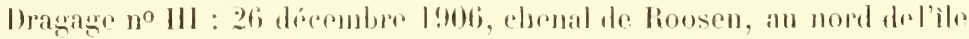

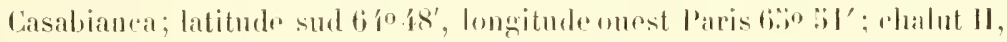

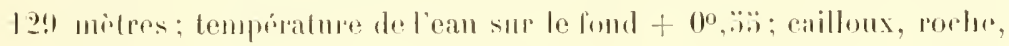
vase, gres rerditre.

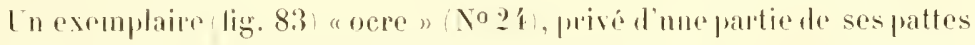
el d'ailleurs en hon chal. Col exemplaime est immatme, mais sos ovigisers peu allomeris eomplemt drjic tomslours ardicles; soncophialothorax mrsure 2mm, ii, r'est-it-dire I millimoitre de mojus que los types de linspece. Ins dillóremes aved ees lypes sont rares et minimes: le tubereule oculaies forme un eome aisn el íleve au-dessus des yeux,

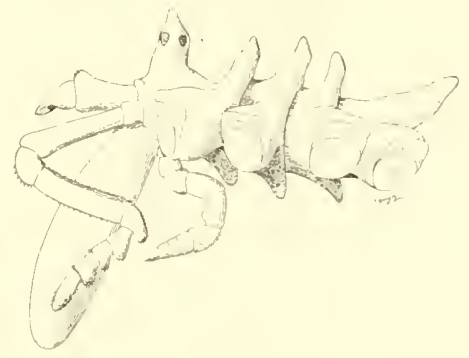

Fig. 8.3. - Immolhea minor Ilodgsun. - Lu

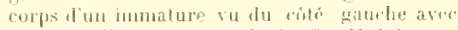
les appendices correspontanls. Gir. 11 1/2. nom unr simple poinlo: l'abdomen est

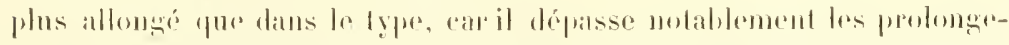
ments cosinx postériens. Les pincess sont dójit réduites it un hourgeron 1$)$.

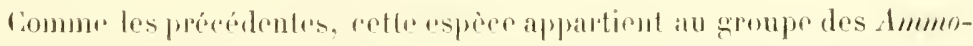

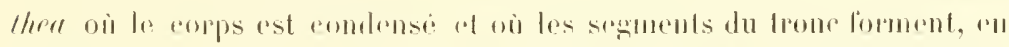

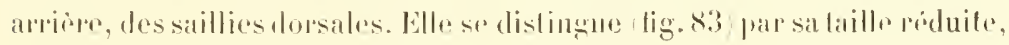


par la linme coniepu do ses sallies dorsales, par sa trompe piriforme ou plutôt longurment ovoïde ì peu près droite en dessous et nettement

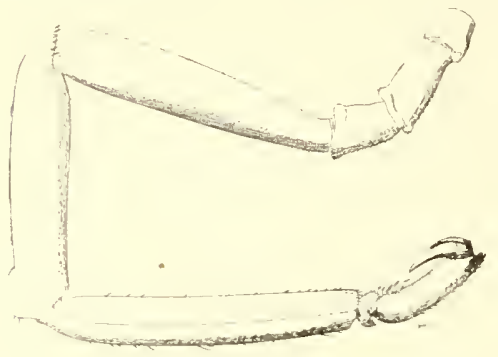

Fig. s4. - Ammolhea minor - La $3^{+}$gratle droite arquér an dessus, par ses pattes fig. 8'́ qui sont médiocrement longnes mais comprimées latéraloment et, par suite, plutòt larges, enfin et surtout par la structure de ses palpes, dont les articles 6 ì 8 sont égaux et très dilatés infórienrement, ce qui donne à cette partie de l'appendice un aspect serratiforme très caractéristique. Le dernier article des palpes est plus long que les précédentset ovoïde; les tingumonls sont partout recouverts de granulations microscopiques, avec eja li quelques courtes soies.

Le type de l'espèce fut capturé prar la "Discovery" dams la région antaretique dr la province australasienne, aux Winters Quarters, par 129) hrasses rtal’ilr Coulman par \&-lï brasses; elle habite ógalement la province de lagnllan. comme lromontre la décourerte du "Pourquoi pas?". Elle sy trome memo dans la zone subantaretique: M. Labille, en ellet. ma sommis un l'yenogonide des Sandwichs du Sud $1911^{\mathrm{a}}$, 114) qui ippartirnt sirement it cette espece: l'exemplaire est un immature dre lat taille du précedent, mais qui porte encore des pinces bien développeres; il fut pris par M. C.-A. Larsen à Visokoi, le 13 novembe 1908.

\section{Ammothea gracilipes E.-L. Bouvier.}

1911. Leionymphon gracilipes E.-L. Bouvier $\left(1911^{\mathrm{b}}\right)$, p. 1140.

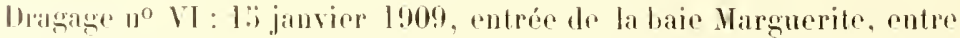

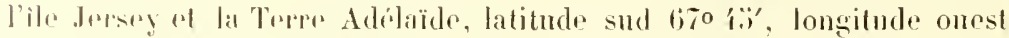
Paris 700 ï; chahul I, 2:34 mitres; température de l'eau sur le fond $+10,18 ;$ porlse, gravior.

Une fempoln adulte, "jame sale ", dont les dimensions se trouvent releries plus loin no $1: 3: i)$. 


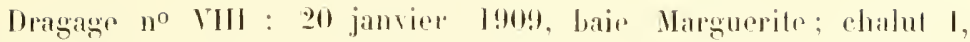
200 mètres, tempríature de l'rin sur le fond $+1^{\circ}, 18$; poche, sravier, vase.

Un male ovigìn "jaune sale "(No 188) ol un jeune "brun sale" No 2:30, ou les ovigieres sont incompletement développés.

La trompe (fig. S.i est identique is relle de l'L. minor, e'est-ì-diare piriforme, tris peu sensiblemput intlichie et un pen plus longne que le céphatothoras ; efle atteint sa largeur maxima (qui ne dipasse pas celle du tronc dans sa partie médiane la plus étroite) un peu au delà du milieu, colui-ci étant indiyué par les traces fort ragues d'un changlement; a partir le ce point des lignes longitudinales se manifristent assir nombremses sur les

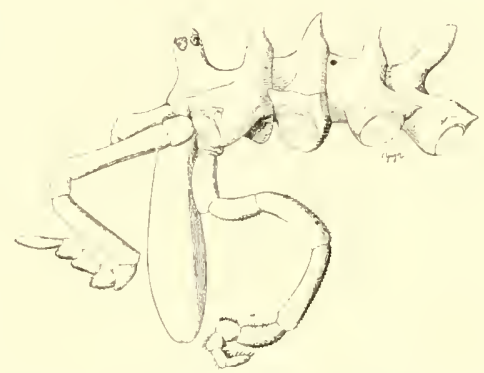

Fig. 85. - Ammothea qracilipes Bouv., o. Inimal vu du colli tranthe aree les appendires andiriturs correspondants, fir. 9. parois dre l'organe.

Le épllallon fig. 8.j) est un peu plus long que large et à preine plus étroit en arriers; son lord antrieur, carrément trontués, porte un líger

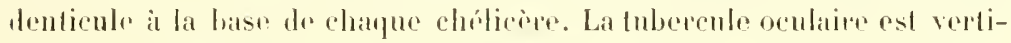
"al, assez haut ret large; it se termine par un cone aign, ou pill une pointe. au-dessus drs yeux. Ces derniers sont largement séparés, trìs nets, peu étendus et ovates; eeux de la paire antérieure sont sensiblement plus grands que les autres.

Le trone tig. 8.i) ressemble tout is finit ì celui de l'A. minnr; ton-

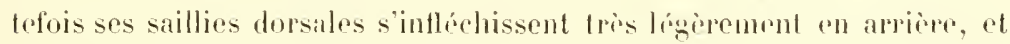
les deux saillies qu'on trouve dorsalement au hout de chayne prolongement roxal sont moins arrondies, plus anguleuses.

Liabdomen (fis. 8.; se dikte peu vers sa partir terminale ou me se dilate pas du tout; dans le jeune exemplaire il est meme rétréci. Sit longurur est ì peupris celle du dermier prolongement coxal; il est heaucoup phus preví que dans l'A. minor, vertical on presque.

Les chélicires (fig. $8 . ;$ ) ne présentent rien de particulier: leur pince. 


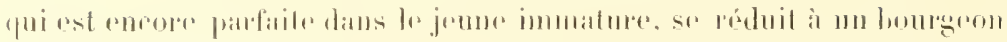
cherz los deus adulles.

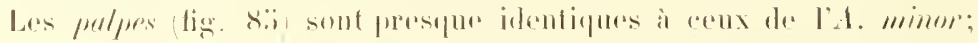

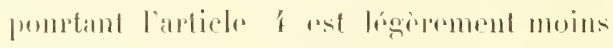

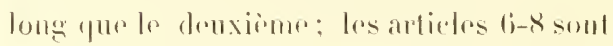
dilatés el donnent à cotte région du palpu me apparenee sematifome.

Les origires sunt egalmont presture idrutigues daus les deux sexes ; chez lo

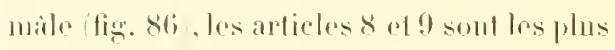
comits at l'article $i$ ast plus citroil gur le fuatriome, contrairemont à ecque lion ob-

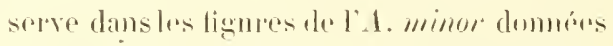

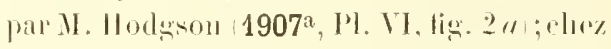
Fir. 86. - Ammolhere gracilipes, o" a femelle fig. 86 , lo dronier article rst tomjours plus lome que le précódent. Iandis

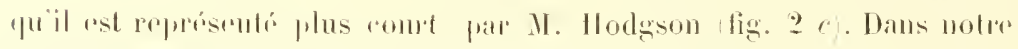

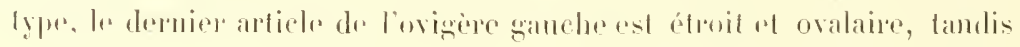
yüil est arromeli commo dans la fignem de 11 . Horloson) daus lovigine opposi.

Les paitles (fig. Si) somt fort dissomblalilus dans les drux espeess: lour demxinue anticle cersal int plus long qua la sommo dos deux articles avoisi-

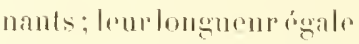
huit lois celle du céplua-

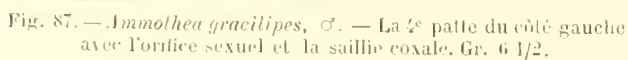
lothorax, or cost à pine si clles sont comprimeres

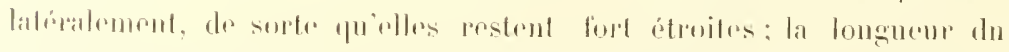

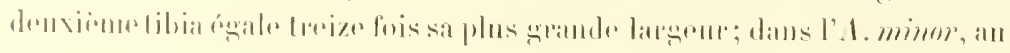




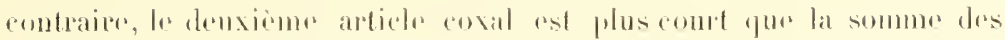

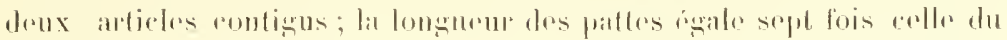

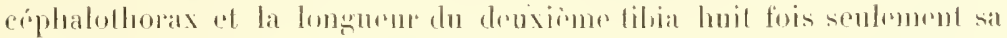

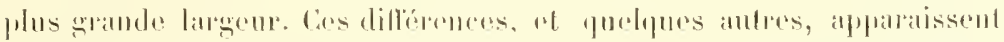

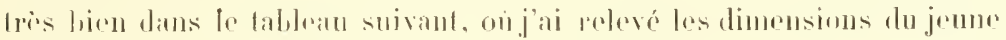

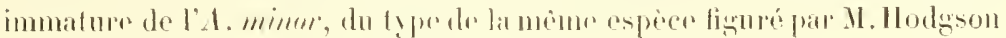

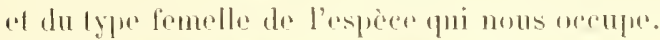

\begin{tabular}{|c|c|c|c|}
\hline & $\frac{1.1}{\text { Immiture. }}$ & $\frac{1}{\text { Type }}$ & $\begin{array}{l}\text { A. GRM:I- } \\
\text { WIPEs " }\end{array}$ \\
\hline 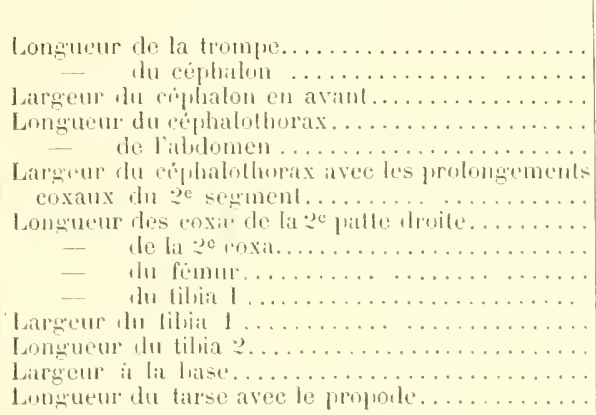 & 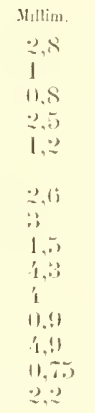 & 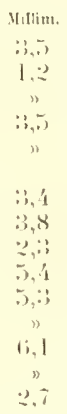 & 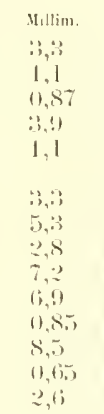 \\
\hline
\end{tabular}

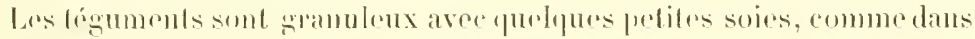

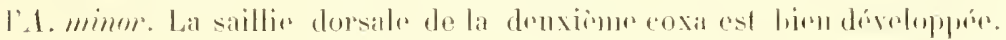

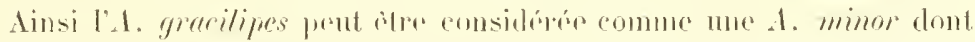

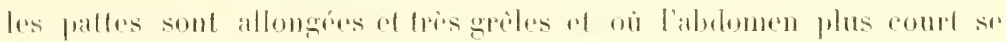

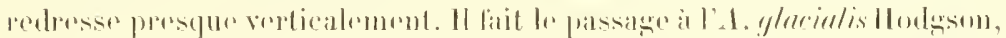

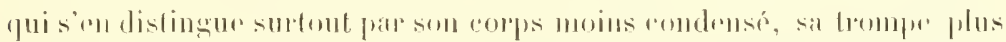

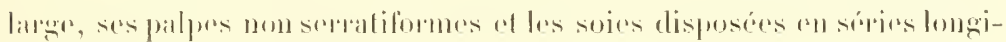
tudinales do ses appendienes.

Ammothea Clausi l'feller.

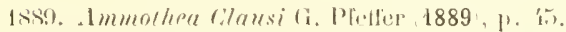

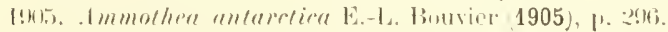

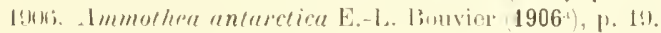

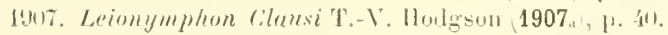


1907. Leimymphon antarcticum E.-L. Bouvier (1906"), P. 56, fig. 37-39, el PI. III, fig. $4,5$.

1911. Leionymphon Clausi E.-L. Bouvier $\left(1911^{\mathrm{b}}\right)$, P. 11 '11.

Lragagne no III : 26 décembre 1908, chenal de Roosen, a u nord de l'ilot Casabianca: chahut II, 1 g! motres; température de l'eau sur te fond + 0 , :ï; railloux, roche, vase, gres verdatre.

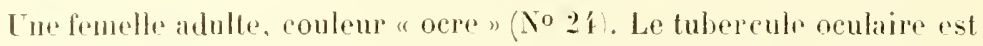
un cone vertical très aigu, l'abdomen un cone obtus légèrement incurvé en arrière; les pattes sont un peu comprimées latéralement.

Dragage no V : 29 décembre 1!008, chemal Pellier, entre l’ilot Getsehy of lile Douner; rhahut II, 92 mètres; température de l'eau sur le fond - 001 ; rase grise, gravier.

Une lemelle "brun jaune " (No fi) oi les orifices sexumb sont assez nets et les ovigires bien développés. Le tubercule oculaire se rétrécil en cone toes aigu au-dessus dis yeux; l'abdomen est vertical, les pattes sont assez comprimées latéralement.

Imagage no XVII : 26 décembre 1909, baie de l'Aniranté, dans l’ile du lioi-Georer, aux Shetlands du sud: chatut I, q20 mètres; température de l'vius sur lo fond $+00,3$; vase, cailloux.

Lne femelle adulle " brun grisatre " No 733 et restée telle dans l'alcool (ammed'ailleurs le type de l'd. antarctica), alor's que les autres rxemplaties sonl complètement décolorés. Trompe remarepablement dilaté elassez fortement trifaciale, tubereule oeulaire en cone vertical tris aigu et assoz rigulier, abdomen verlical, cylindrique à la base, en cone obtus at sommet; les paltes ne sont pas sensiblement comprimés.

Je releve ici quelques dimensions des trois exemplaires précédents,

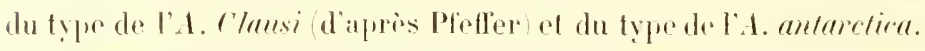

\begin{tabular}{|c|c|c|c|c|c|}
\hline & "A. CLACSI". & $\begin{array}{c}\text { "A. INTARC. } \\
\text { TICA". }\end{array}$ & 10 24 & $N^{\circ} \mathrm{i} i$ & $x^{\circ} 733$. \\
\hline 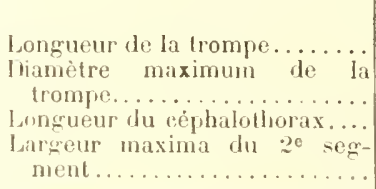 & $\begin{array}{c}\text { Millim. } \\
4,5 \\
n \\
n \\
5\end{array}$ & $\begin{array}{l}\text { Mithin. } \\
5,1 \\
1,9 \\
5,1 \\
4,8\end{array}$ & $\begin{array}{l}\text { M1llim. } \\
7,9 \\
2,2 \\
-4,3 \\
1,3\end{array}$ & $\begin{array}{l}\text { Millm. } \\
5,3 \\
2 \\
3,8 \\
3,4\end{array}$ & $\begin{array}{l}\text { Milim. } \\
5,2 \\
2,2 \\
4,1 \\
4,2\end{array}$ \\
\hline
\end{tabular}




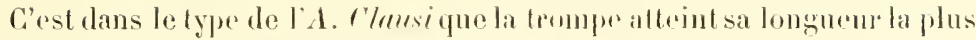
laible; mais cet organe n'est pas sans présenter des variations ot, comme fres autres parties du corps, on l'a wn phus haut, ue sont pas sans varier alles-mèmes, les différences que javais relevées jadis $1907,09 !$ entre

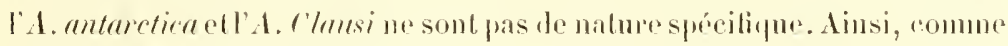

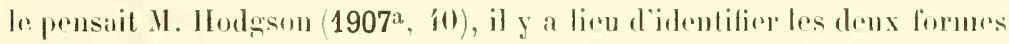
et de consepver à l'espion le nom demné par Ploffere.

L'A. Clansi appartient an grompe oì les segunents du tronc ne presentent

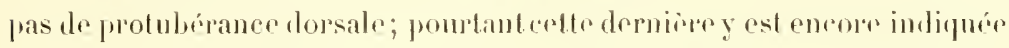
l'all une saillie médiane aiguë de's phus nettes. L'aspiec est remarquable (lig. 8.) parla grande condensation de son copps, par son aldomen verlieal el presque atteint à sa base par le hord postérieur du troisième segment,

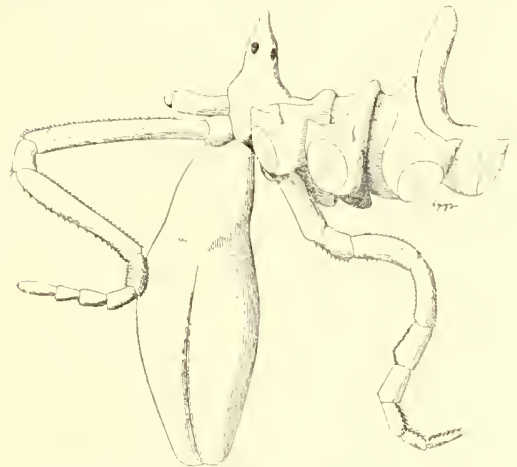

Fig. 88. - Ammolhea Clausi Pfeller, o. - Le corps du colts gauche, aver la chélicere, lo palpe el lovigere correspontants. Gr. 9 .

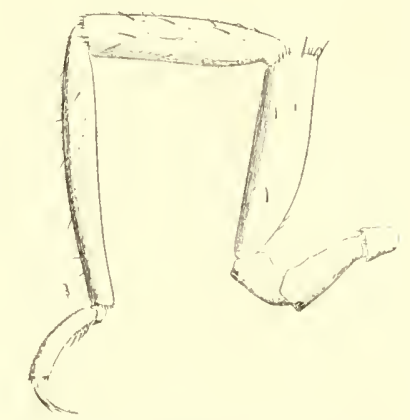

lig. 89. - Ammothea Clausi Pfeffer, f.La $3^{\circ}$ palle grach" arec l'oritice sevurl. Gi. i.

parr sa forte trompe piriforme, on le tiers basilaire moins large forme mne sorte de pédoneule qu'un sillon d'étranglement sépare de la parlie dilatée, par ses palpes où le deuxieme article rest d'un quart plus loug que le quatrieme, par les deux angles saillants et aigus de ses prolongements coxaux dans tem bord disfal, ot par la grande dimension des grilles auxiliaires qui égalent au moins les deux tiers de la grillo principale. Les palpes ne sont pas serratiformes dims leur partie terminale, "t la partie dilatée de la trompecest plus ou moins trilaciale. Il y a sur les pattes lig. 8? quelques fortes soies fuarses et d'intres très petites, tris nomlirenses en 
dehors de certaines lamdes longitudinales absolmment nues. Le premier article coxal rst beauromp plus court que le troisième, le denxième égalant en longueur los denx antres. Commo dans toutes les Ammother, la saillir médiane dorsale de cet article est fort apparente. Le fémur est un peu plus long que le tibia 1 et beaucoup plus court que le tibia 2. Le front est carróment tronqué avee une saillie aiguë à chacun de ses angles; te tuberente ocolaire se fermine tonjours en un cone haut et tris pointu.

L'espècen'est pas comnue jusqu'ici en dehors de La province de Magrllan; alle y fut découverte à la Géorgie du sud, et II. Charcut l'a retrouvée aux Shetlands; mais olle rst également antaretique, ainsi tue le montrent les captures du "Francais" (Port-Chareot) et du "Pourquoi Pas?".

L'espece n'est pas sans affinités aver l'A. glucialis IIodgson, mais elle se rapproche surtont de I'A. anstralis Ilorlgson 1907a, A6, Pl. VII, fig. I), yui s'en distingue toutefois par son tubereule optique arrondi, par son abdomen oblique é íloigné du troisieme segment du trone, on outre par son peritement sétifiere bien plus riche. L'd. anstealis lut peiso par la "Diseovery" dans les eaux antapelipues dre la province australienne.

Genre Achelia Hodge (.1 mmothen des auleurs).

Les Achelia se distinguent des Ammothea par lour petite taille, leur corps ou les articulations segmentaires dispraraissent à divers degrés, par leurs palpes de huit articles (1), par leur fómur qui présente distalenent une protubérance oì s'ouvent des glandes cémentaires, et par le tubercule au sommet dnquel s'oure le pore génital des màles. Elles ressemblent par leur petite taille aux Immothellu, qui présentent d'ailleurs des chélicires dont le scape a deux artieles, avee les articulations segmentaires, les palpes de neuf articles dis peut-êtee d'après M. Cole) (1904, 273) ot les fémurs non glandulaires des Ammothea. C'est, par suite, avere raison, que 11 . Verrill, en 1!900, a ćtabli le genre Ammolhellu, dont 11. Cole (1904, 27:3 a domné une exeellente diagnose.

L'espiece typer lu gente Ammollefluest l'A. mgulowa Verrill, qui semble bien identigue à une espèee plus ancienne, l'dumothen appendirmluta

(1) Les palpes n'auraient que 7 articles dans l'dehelin apocilis Verrill, donl les mâles présenten\} d'ailleurs des fulnercules sexumes comme ceux des dehelin. 
Dohrn, que M. Cole place justoment dans le grenre Immothello: Il. Cole a rémin anx Ammothelles l'Ammothen bimmgnemhlu du memo anteur, mais

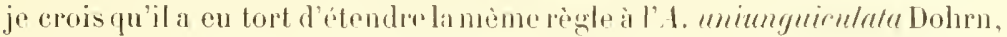
dont le corps n'est pas compliement segmenté et dont les palpes ne comptent que sept ou huit articles. Comme il a décrit d'ailleurs 1904

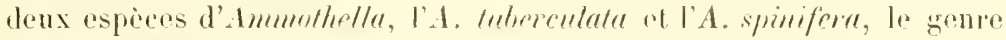
établi par M. Verrill comprend à eomp sùr quatre esprees. A celles-ci on doit ajoulor, il me semble, l'Ammothere hespiete Dohm (. maynirmetris Dohon) el l'Ammothen Hoeki l'feller; coltw derniope espore provient de la

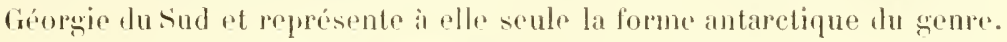

On a vu plus haut que l'Ammothen cureulen Bouvier, dn "Francais ", est le jeume d'une fmmother prostue certainement le l'A. gibbusa), ot jo montrerai plus loin que l'A. affmis Bouvier, do la mòne expredition, doil èter considéré comme l'ótat jeume del'A cheline commmmis.

La rénomination de co genme a subi des vicissitudes nombreuses:

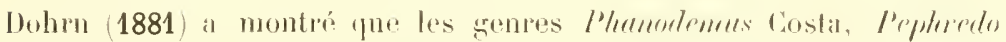
Foodsir, Eudeis Philippi, l'letycheles Costa, Aleimmes Losta ot les Ammo-

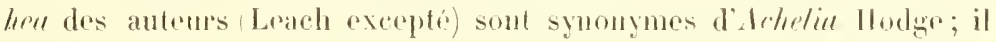
rusail mème, et pent-ètre à juste titer, que cotte synonymie doit s'rlandre au gente l'usithop foodsir, encore que tumifuc espece de ce gener,

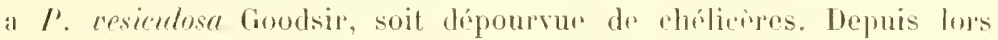

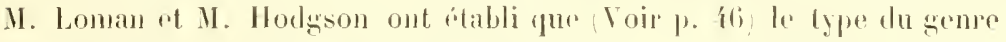

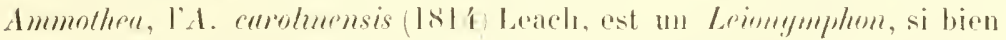

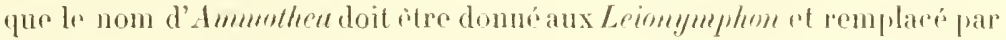
celui d'Aehelin.

M. Ilodgson 1910, 亿:36) adople colte nouvelle dinomination, mais il

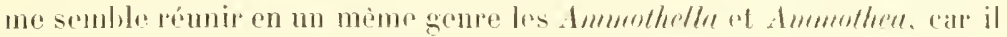
attribur à ses trohelia des palpes de Inuit on neuf ardicles.

Dans le geme Actedir lui-mome, les difticultís somt nombrenses a cause de la synonymie des espèers; antant yu'on morut jugre, ees dernives se riduisant actucllement à 17 :

1. vulgaris Costa 1801 (franciscann Dorhn ls81). Niples.

A. uniunguiculata Dohrn $18 \$ 1 . \ldots \ldots \ldots \ldots \ldots \ldots$ -

A. Langi Dohrn $1881 \ldots \ldots \ldots \ldots \ldots \ldots \ldots \ldots \ldots$.

A. echinata Hodge, 180, breripes Hodge, 
achelioides Wilson, spinosa Wilson, fibu-

lifera Dohrn, pycnogonoides Qualtef., spini-

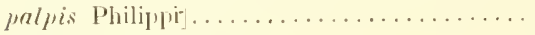

A. setulosa Loman $1912 \ldots \ldots \ldots \ldots \ldots \ldots \ldots \ldots$

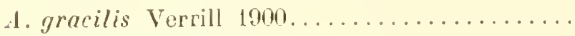

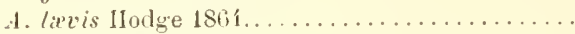

1. srabru Wilson $1878 \ldots \ldots \ldots \ldots \ldots \ldots \ldots \ldots$

A. burealis Schimkewitsch $1895 . . . \ldots \ldots \ldots \ldots$

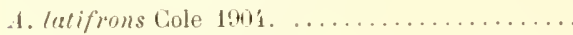

1. mibilopensis Cole 1904.................

1. alaskensis Cole 1904..................

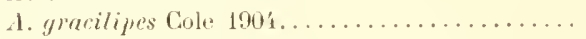

A. superbu Loman $1911 . . . \ldots \ldots \ldots \ldots \ldots \ldots$. . . . . .

A. assimilis Haswell $1805 . . . \ldots \ldots \ldots \ldots \ldots$

1. Grevicauda Loman $1908 . . . \ldots \ldots \ldots \ldots \ldots$. . . . .

A. nana Loman 1908 . . . . . . . . . . . . . . . .

1. socors Loman 1908 ...................

1. Wilsoni Sohimkewitsch $1890 \ldots \ldots \ldots \ldots \ldots$

1. magniceps Thomson $1884 \ldots \ldots \ldots \ldots \ldots \ldots$

A. communis Bouvier $1906 \ldots \ldots \ldots \ldots \ldots \ldots \ldots$

Toutes ces rspèces sont littorales ou sublittorales. Deux aspèces habitent les mer's suliantaretigues : l'A. lire is llodge, qui se trouverait, d'apris Böhm (1879, 186), aux Kersuelon, et l'd. Wilsomi de la province magellanique; ot deux les mer's antaretiques, l'A. communis à l'A. serratipalpis. Les drux dernieres ont été reeueillires par le "Pourquoi Pas?".

Achelia serratipalpis E.-L. Bouvier.

1911. Immothea serratipalpis E.-L. Bouvier (1911 b), p. 1140.

Dragager $1^{\circ} 111$ : 26 déecmbre 1!108, chenal de Roosen, an nord de l'ilot

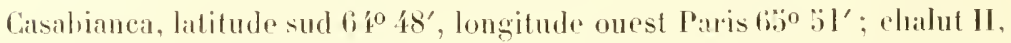
129 metres; trmpérature de l'eau sur le fond + 0 o,, $3 ;$; cailloux, roche, vase, gress verdatre.

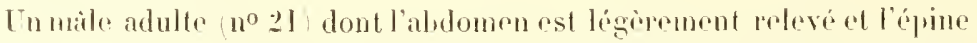
coxale remaryuablement longue, birn plus longue que le premier anticte coxal où ulle est placée. L'orifice glandulaire, situé dorsalement au milieu de l'article coxal suivant, rst des plus nets.

Dragagere $10^{\circ} \mathrm{V}$ : 2!! décembre 1908, chenal Pellier, entre l'ilot Gutsehy

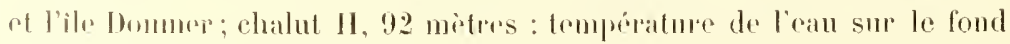
- $0^{\circ}, 1$; vase grise, gravier. 
The femello adulte "janne" (No 12); l'épine coxale n'est pas plus longue que l’articlo qui la prorte: l'alidomen est ì pen près horizontal; los orifees glandulares dorsaux de la deuxidue coxa sont très apparents.

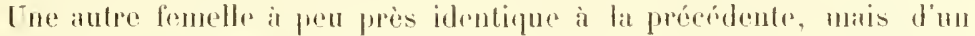

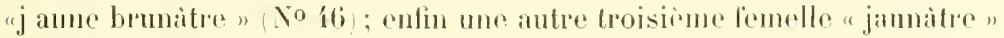
(No 43), oủ l'abdomen rst rudimrntaire, fous les autres caractires b́ant normaux.

Les dinensions de l'esperen sont les suivintes:

\begin{tabular}{|c|c|c|}
\hline & of $x^{\circ} 21$. & \& $x^{\circ} 42$. \\
\hline 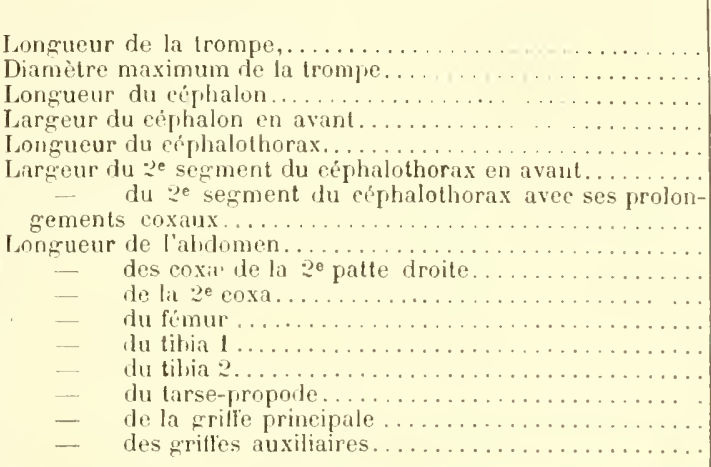 & $\begin{array}{l}\text { Mulim. } \\
1,90 \\
0,5.5 \\
0,50 \\
0,75 \\
2,10 \\
0,07 \\
2 \\
1,10 \\
1,70 \\
1,30 \\
3,30 \\
3,30 \\
3,70 \\
1,62 \\
0,88 \\
0,40\end{array}$ & $\begin{array}{l}\text { Mijlim. } \\
1,9 \\
0,62 \\
0,45 \\
0,78 \\
2,10 \\
0,56 \\
1,65 \\
1,30 \\
2,50 \\
1,50 \\
3,10 \\
3,30 \\
3,70 \\
1,12 \\
0,86 \\
0,14\end{array}$ \\
\hline
\end{tabular}

Cette espèer est remarquable par son corps peu condensé of ses patles plutit greles, surtout par la partic terminale de ses palpes, qui est fortement serratilormo.

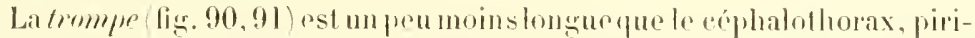

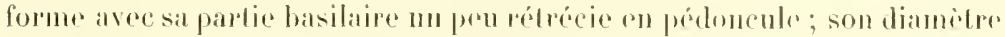
maximum se trome un peu avant le milien ef noftre quo des dimrosions

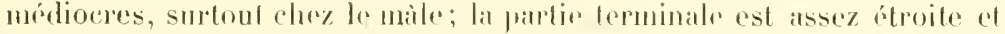
oblust.

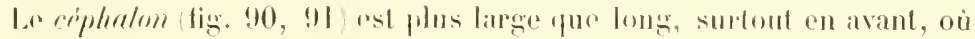

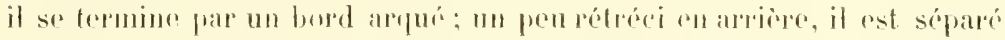

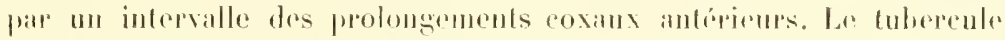
oculare, assez large, est ì peu pros aussi haut pue la bouguene du cópha- 
Ion; dilaté a la base, il devient ensuite subeylindripue, puis se termine par une voussure hasse et murronée. Les yeux sont pelits al largeurut

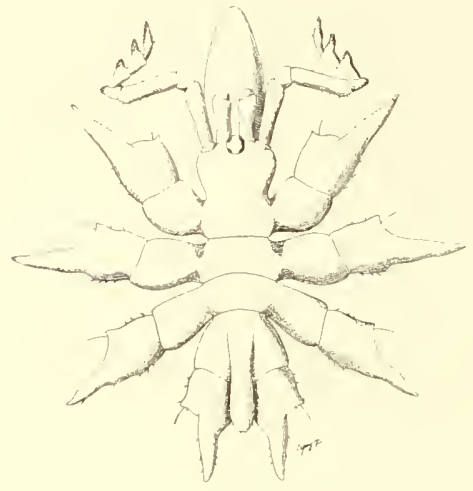

Fir. 90. - Achelia serratipalpis Houv. O". Exemplatir va lu cinte dorsal avee les rheliceres, les palpes el la base des pattes. fir. 11 1/2. séparis. Le fubercule est presque vertical; il se tromse immédiatrment en arrière du bord anterieur du céphalon.

Le trome fig. 90, 91) se rélrécit régulièrement d'avant en arriere: ses segments sont séparés, très nottement articulés ot à peine ronvexes dorsalement. Les prolongements coxaux sont plus longs que la largene des segments qui les porte, assez éloignés les uns des autres et étranglés à la base; du coté dorsal, ils prósentent distalement une paire de bubereules inigaux, plus diveloppés chez te male.

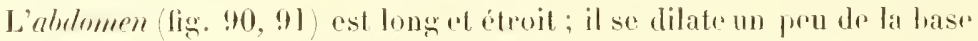
an sommet, puis se rétrécit en un hout ohtus. Horizontal on un pen melevé, il atteint et parfois mème dépasse l'extrémité distate de la prer-

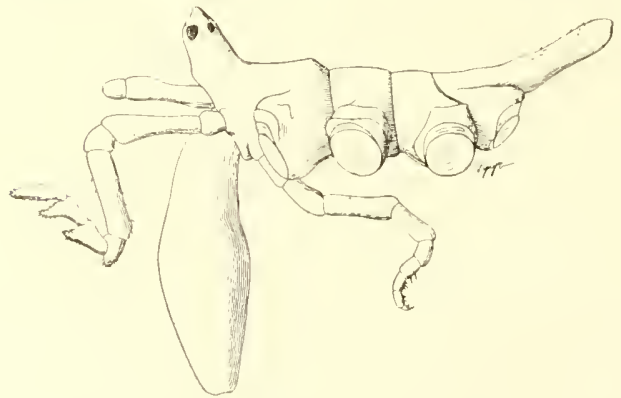

lig. 91. - Achelia sermalipmlpis, ९. - Animal vu du coil gauble avec les appendices ciphaliques correspondints. Gr. $111 / 2$. mière coxir. Dans la femelle du Yo.43, l'abdomen est réduit à une simple saillie.

Jes chélicères (tig. 90, 9) n'atteignent pas tout a fait te milien do la trompe; lemr seape rst un peu dilatio en avant. leur pinee riduite à 111 bouton.

Les palpes lig. 90,91

dépassent la trompe presque de la longueur de leurs quatre derniers articles. Les articles 2 et $i$ sont subégaux; les trois articles suivants 
sont courts, chloits à leur base, puis dilatís en languette sétifire en avant ef en dessous, er qui domne aux palpes une apparence serrati forme tres caractiristiqur; Ir huitiome est un peu plus long pue les précédents, étroit, ovalaire et égaloment muni de courtes sojes.

Les arigires tig. 92, 93 ne prosentent rien de particulien: leurs deus arlicles les phus allonares, les quatrieme ot cinquieme, sont à peu près égaux; viont onsuite le deuxieme; les sixieme el septiome sont subegaux, les autres phus ridnits, surtout lo dernier, yui est tubereuliforme. Les apines diflérenciéns des

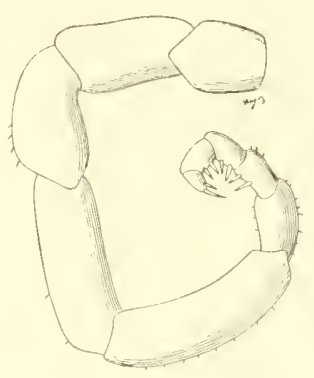

10ig. 92. - Ichelua serratipalpis, Fig. 93. - Ichelia serratipalpis, o. - Migite droit. Gr. q2. $^{2}$

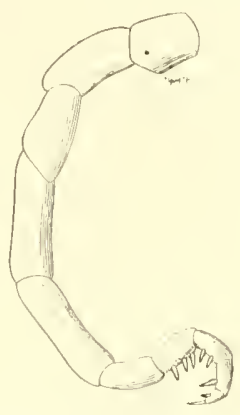

?. - Ovigise droit. Gr. 49. quatre inticles terminaux sont toutes fortement et longuement denticulies elez le male fig. !), sauf' la derniere, qui a l'aspect d'une carifle. Au surplus, les wrigires du mâle sont heancoup plus robustes que cenx de la fromelle tig. 93 .

Saul me forte saillir spiniforme qui oceupe en dessus l'extremite

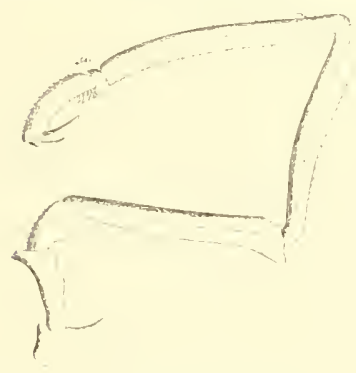

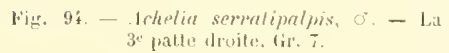

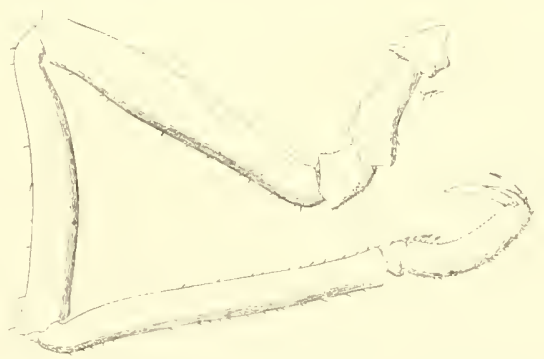

Fig. 93. - Ichelia serratipalpis. P. - La ir patte troite aves lorilice suxuel el la sallie eoxale. Gir. 7 .

distale de la premiipe cosa, les pattes (lig. 9 $\{$, 9:i) sont complitement

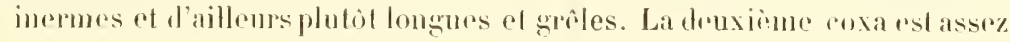


fortement rétrécie it la base et anssi longue on un pen plus longue que les deux articles coxaux contigus; son orifice glandulaire dorsal se trouve un pen au delì du milien de l'artiele, sur une petite saillie. Le fémur et le tibia 1 sont subúganx, le tibia 2 rtant nu peu plus allongé. Au tarse trìs rourt fait suite un propode arqué assoz long ef peu rétréci distalemenl; il y a une épine sur le tarse ct deux on trois analogues dans la partie aroisinante du propodr. La grifle principale égale à pen pres les deux Lims de en deruier apticle; elle est acempagnere de deux fortes griffes auxiliaures moins longues de moitic. De coutes soies éparses ou peu régulièrement distribuées se trouvent en assez grand nombre sur les divers articles des pattes; la lorievetó et la linesse de ces soies sont telles quo los pattes semblent nues au premier examen.

Les pattes des males fig. y) se distinguent de celles des femelles

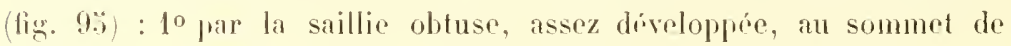
laquelle s'ouver l'orifice sexuel sur la deuxième coxa des deux pattes postípieures; 20 par l'épine dorsale beaneoup phus forte de la premiere coxa; $3^{\circ}$ par la ditatation un pen plus grande du fémur. La saillie dorsolistale de ce dernier article est un cone à la pointe duquel s'onvent les slandes cémentaires.

A/finités. - Cefte espèce est évidemment l'me des phus primitives ef peut-itre la plus primitive du genre Achelia, car clle a les pattes plus wrôles of plus longues, le corps plus étroit, la segmentation plus parfaite ef l'anmature épineuse plus réduite que toute autre esperen. La forme prartieuliere de ses palpes lni domne, en ontre, une place à part dans le genne et ha raproche des Ammothées à palpes serratiformes.

\section{Achelia communis.}

I9H. Ammothen communis E.-L. Bouvier 1906*, J. 20.

1967. Ammothen communis E.-L. Bouvier (1906 ), p. Á.

1717. Immothea affinis E.-1. Bouvier (1908), p. 50, 1ig. 33-30 (juv.), Pl. III, fig. :3 el tig. 23, 32 du lexte.

19ms. Ammother communis T.-V. IJodgron (1906), p. 1i2, I'I. II, lig. 1 el 1 a.

1911. Ammothen communis E.-1. Bonvier $\left(1911^{\mathrm{a}}\right)$, P. 1140.

Mragage no III : 26 décember l!08, chenal de Roosen, an nord de

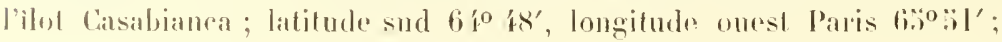


chalut II, 129 metres ; température de l'eau sur le fond + $0^{\circ}: 33$; caillonx, roche, vase, gres verdatre.

Une femelle adulte (No 21 an compagnie d'une A. seratipalpis. 10 octobre 1909, Pont-Circoncision, dans l'ile Petormann. Lne fenolle? adulte tronvée sur une Mgue lrune Drsmarestia par it metres de fond; les glandes dorsales de la deuxieme coxal y sont tres apparentes (No 433$)$.

16 octobre 1909: plage des Rookries, dans l'ile Petermann.

Denx jemes " trouvés à marée talsse, sur les galels d'une pelite plage;

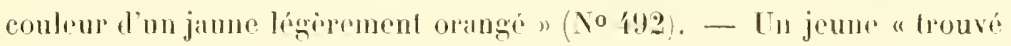
sur un gros galet, a marée basse, en un endroil de la plage qui ne déconvre pas $\left(0^{\mathrm{m}}, ; 0\right.$ d’eau) ) (No 493$)$. - In màle " trouvé à marée hasse sur un galet, sons me colonie de Bryozoaires $\left(0^{\mathrm{m}}, 60\right.$ d leau $)$. Conleur

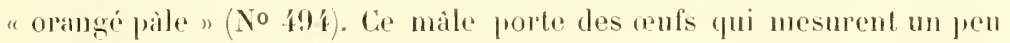
plus de $100 \%$.

30 octohre 1909: mème localité. Une trentaine d'exemplaires "récoltés a mamo lasse... Ils se trouvent principalement, el en grande quantití, sur les cailloux qui ont de nombrenses colonies de bryozoaires et d'Anflo-

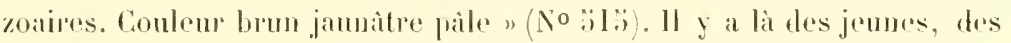
femelles et beaucouj de miles wigères dont les aufs mesurent $100 \mu$.

3 oetobre 6!0!: plage de l'ile Peternamn. Cone trentaine d'exemplaires des deux sexes. Ces Pyenogonides, observe M. Gain, sont " nombrenx a la partie inférienre des cailloux al zalets, sur lesquels sont fixés des Bryozoaires el Coralliaires. Conleur jaunatre " (No 537 ).

fer novembre f!og! : deux femelles et mu màle ovifere "ripportés par M. Lionville de la plage de l'ile Petermann; trouvés à la face inlérirum des calloux, parmi los spongiaress (No 3606 ).

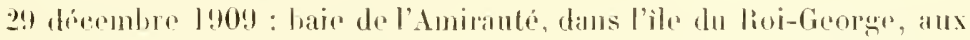
shethlands du Sud. Quarante exemplaires muviron, dont la moitie de mâles pour la plupart ovifirms. "Trouvés sur lis rochers d'une plage de l'anse est de la baie " ( \o 706 ).

Cette esprece est noltement caractérisér far ses palpes un peu serraliformes, ou ledernier article est étroit el plus long que les précédents; par la segmentation du trone, qui est toujours fort nette entre les trois preExpedition Charcot. - Bovrer. - Pyenogonides du " l'ourquoi Pas?". 
miers segnents, mais variable et parfois nulle entre les demx demiers; par les denx tubereules silues dorsalement au bout des prolongrments coxaux th par les deux sallies spinilormes qui oceupent dorsalement frextrémite distale de la premiere coxa. Les antres parties du copps ot des appendices sont depourves de saillies; te denxieme artiele eoxal est conrt, presque globuleux chez la femelle of en massue chrz le màle; on peut assez fréguemment y apereresoir du coté dorsal l'orifice shandulaire, qui neest point porté sur une sallie.

L'A. emmmunis mérite larement le nom que je lui ai donné, ear clle fullule aux points oi elle lut déourertepar le "Français " haie des Fandes, ile Booth-Wandel ot ile Wiencke of ou l'ont trouves a leur tour les naturalistes du "Ponrqnoi Pas?" On in vu plus haut que ces depniers l'ont prise an abondanee, non seulement en pleine zone antaretiqne, mais anx Shethands dusud, qui šbignent davantage du pole. Liespèce est dene largenent represention dans les raux antaretipues de la provinee magnlanique, wì elle se frouve à la coilr, rarement à une certaine profondeur

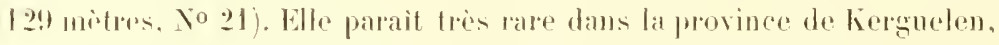

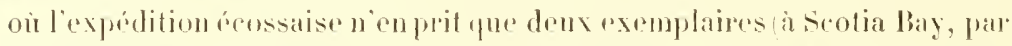

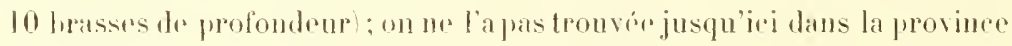
australieme, et 17 . Ilodgson me la mentionne jas dans sa belle étude sur lestryenogonides do la "lliscovery n.

Cefte exper presente qualques variations, entre autres dans ses prolongements coxanx, 4ui peuvent etre contigus un sépares dans la saillie des tulwrentes qui se trourent au lout de ces prolongements of dans la direction de l'ahdomen, qui peut atre phus en moins releve. Ces variations

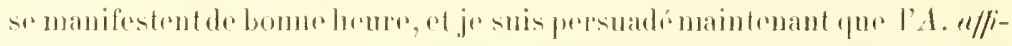
nis louvier du "Fangais " represente simplenent un stade jeune de lis. communis.

Genre Austrodecus Hodgson.

Dans ee genere, latrophie des chélicères est complete comme datns les

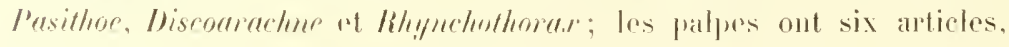

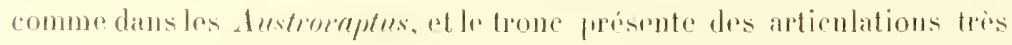
nettes commedans les Trygieus. 


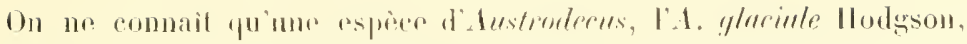

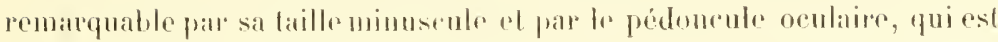

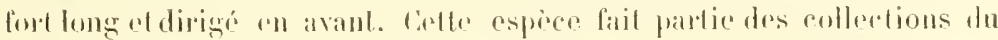
"l'ourquoi P'as?".

\section{Austrodecus glaciale Ilodirson.}

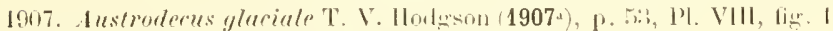

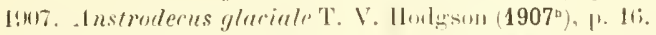

1911. Iustroterus glariale E.-L. Rinuvier $\left(1911^{\mathrm{b}}\right)$, 1. 11:0.

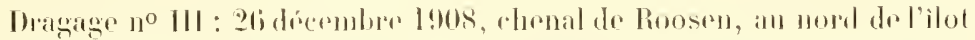

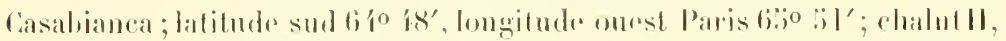

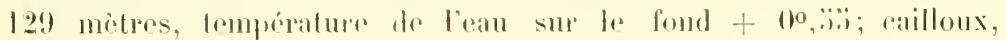
rocher, greses verdatre.

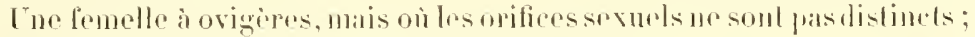
lo céphabothorax mesure emiron $1 \mathrm{~mm} .3$ ( No 33 ).

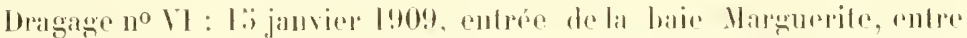

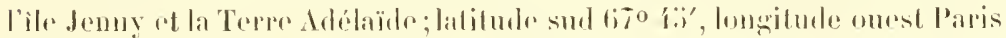

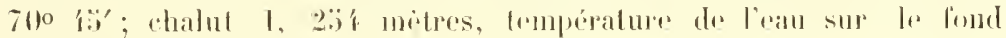
$+10,18$; roche, gravier.

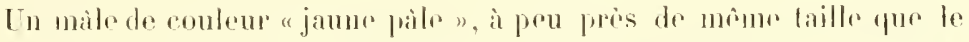

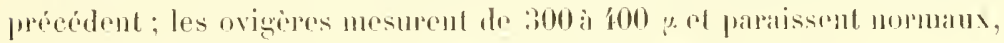
mais je nai pulaperervoir los orificessexuels (No 1:3i).

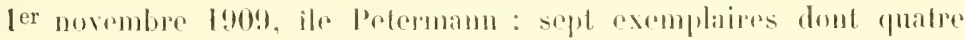

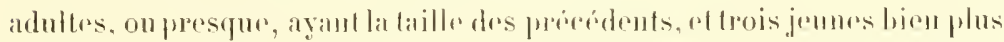

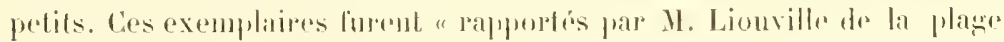

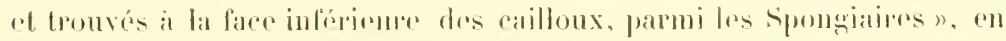

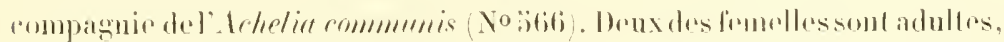

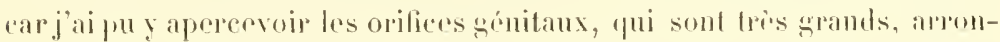
dis, of situes vers le milien de la fare vembale de la deuxieme coxadres

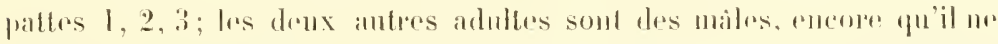

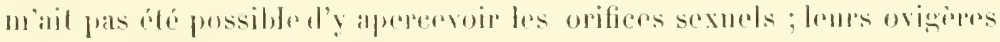

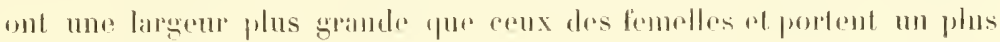
grand nombere d'epines.

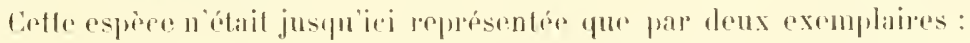


me fomelle type tromvón parla "Discovery" ans Winter Ouarters do la bain Mac-Murdo, par moins de J0 hrasses, - el m jemmr saus ovi-

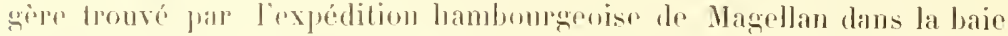

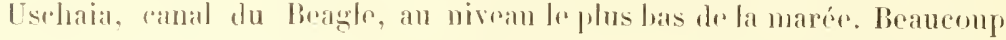
phe riches, los captures du "l'omrquoi l'as?" permeltent de comblem

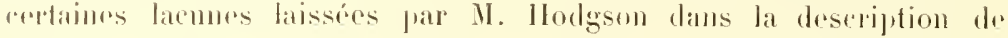

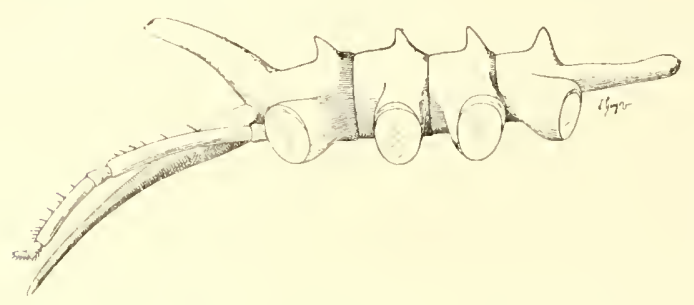

Fig. 96. - Austrodecus glreriule Hodgson \&. - Animal vu du cilti gauche avec les pralpes correspondants. ce runieux Pyenogonide.

Jobserverai tout d'alood puolesexemplairesdu "Pouryuoi Pas? " (lig. !6j) présentent tous les calactions ressentiols de lispirce: allongement hizarere des pédoneules oculaibes, gui ressemblent à mo rostro de

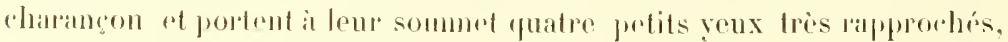
fulereules dorsanx segmontaires, gracilití de la trompe dans sa partie

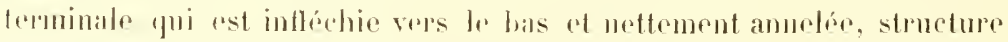

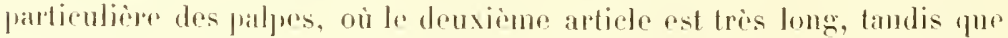

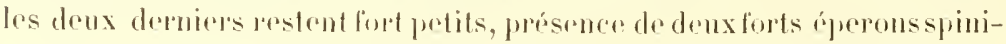

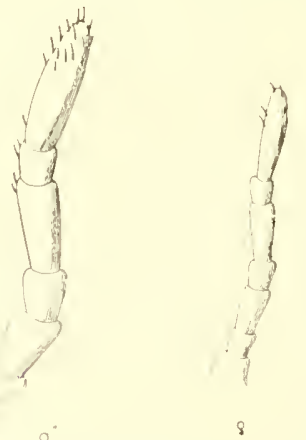

lig. 97. - Austrotecus ylariale. - 11-i-

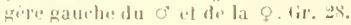

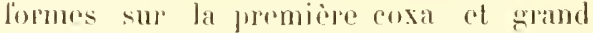
allongement des propodes yui sont un peu alrejurs et dépourvos de erandes ipines.

Lesorigeres fig. 9i/se romposent toujours de six atticles, comme ceux de la femelle étudiéc jar M. Hodesson, mais on peut se demander, avee ce dernier auteur, si le pédoncule assez lomg qui les porte n’a pas lat signification d'un article. Hams les denx sexes, les frois articles basilaires sont comts mais imégax, lo deuxièmo ritant de leateoup lo plus grand: lo quatriome

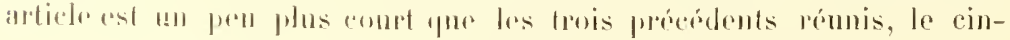




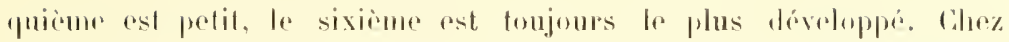
les moiles, les ovigros sont robustes ot prosentent sur la moitie temminale

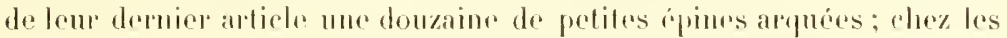
lemelles, ils somt aussi longr, mais plus groles, et lom anmature se limito à deux ppines terminales. En tout cas, les ovieniers somt lort petits dans

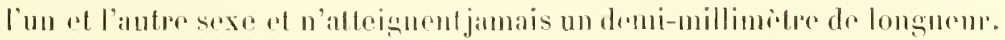

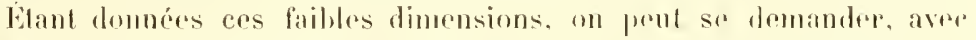

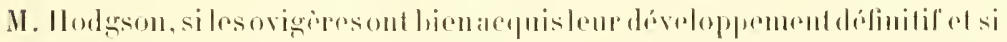

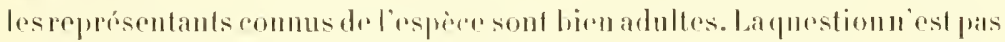

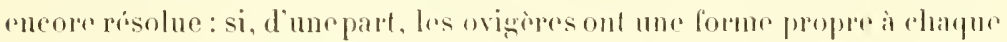
sexe, et si l'on apercoit les orifices srexuls rloz les femelles, il est hom de noter qüil ma été impossilule d'aperervoir cos dernirrs chez lusmalrs ol quw, môm chez les lemelles, oil ils sont fris grands, jamais je n'ai fucomstater leur presenecsur los patles de la dernière paire, où, pomtant, les signale M. Ilodgson. Je crois, mn lait, que les rxemplaimes doul j’ai pu lixer le sexr, de mimm que la lomellede H. IIodgson, sont des individus bion pros didreadulles.

Comme on l'a vil plus haut, l'Austrmelerus glaciale est une esperce littorale ou sublittorale, antaretique et subuntarelifur; elle est probablement répandue partout aulour du pole sud, bien qu'on ne l'ait pas encore signalé dans la proviner de kerwuelen. Elle riest sans doute pas rare,

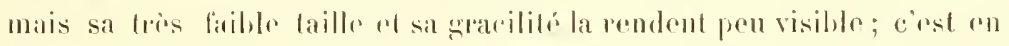
triant avee soin $m 1$ lot d'Achelie emmmmmis quo j’ai pul trouver les stpl exemplaires du vo ititi.

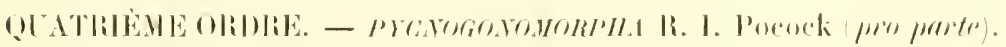

\section{Famille uni,pue. - PYCNOGONIDA.}

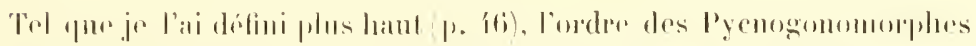

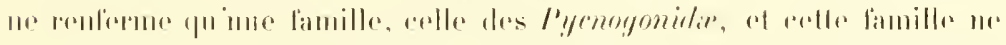

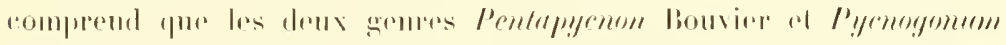

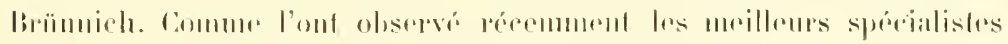

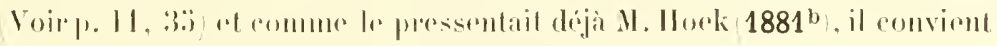


d'altribuer à mphónominn de convergence la disparition des chélicires, despalpws desovigìnes dans les Ploxichilidés ef les Pyenogonidés ; c'est

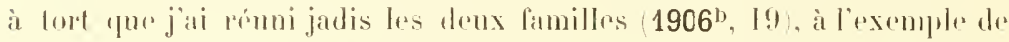

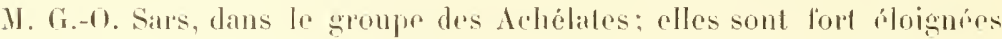
l'une de l'antre ot apparliement sans conteste à deux ordes diffórents. Est-il naturel te rómnir aux Pyenogonides, cumme l'a fait It. Loman

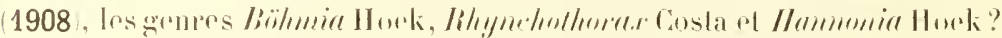

(y)amd on exannine les caractires que 1l. Loman attribue a la fanille

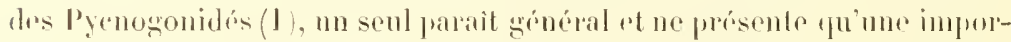
taner médiocere, puisqu il est simplement relatil à la lorme du corjs, me forme qu'on retrouve an surplus ailleurs of notamment chez beaucoup d'Ascorhynchomorphes. Il est rai qu'on pourait considérer la famille des Pyenogenidis, ainsi eonçue, comme une lamille par chehainements, on l'on partirait de formes primitives, lellos que les Bölumia, pour arriver

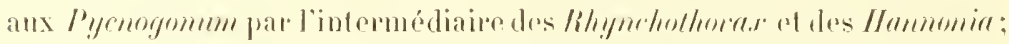
maris alor's, quelle serait la place des /Prulapyemondans cefte famille s'il est exact, comme je crois l'aroir élahli plus haut p. 2 I-2ii), que les formes dicapodes sont plus primitives que les formes acheprodes? Les Bölumia, Rhyn-

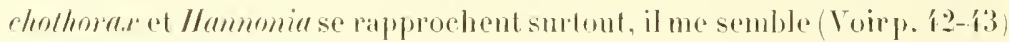
des Ascorhyonehomorphes; et, dans tous les eas, il me parait sage de

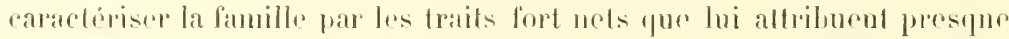
tous les anteurs; olle se limitera ainsi aux deux gemps l'emapyrmm el

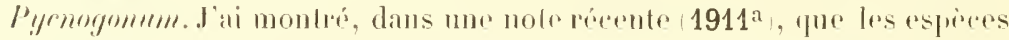
appartenant à l'un of l'antregenpe preuvent se diviser en deux gronpes suivant pure lenr surlace chitineuse est thagrine, ou lisse aved des lignes texzmmentaires difléremment pigmentées equi dessinent un réseau.

\section{Genre Pentapycnon Bouvier.}

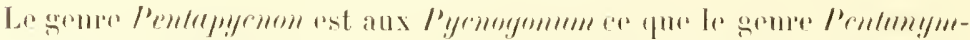

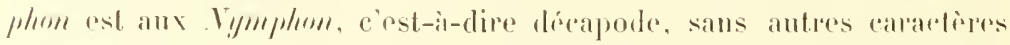

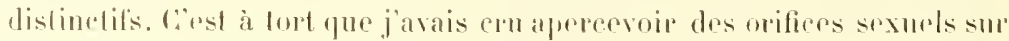

1. Vuiri les caracteres altribués par M. Loman à la famille des l'ycnogonidés : "Corps épais,

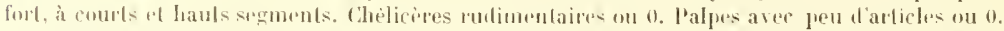
(1)igeres aver 11 article's ou moins, leurs articles lorminaur munis d'épines ou de foils 1908. 


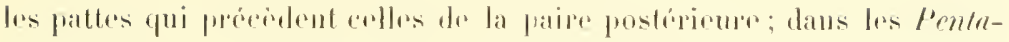

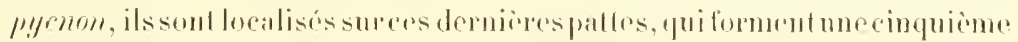

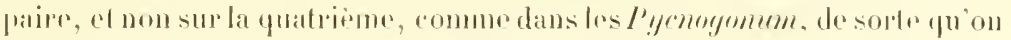

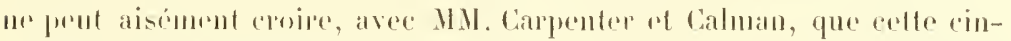

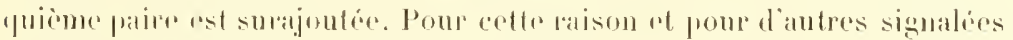

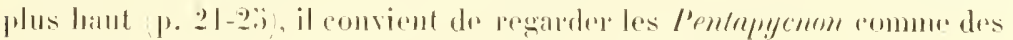
types primitifs qui out conservé le caractere ancestral déeapode.

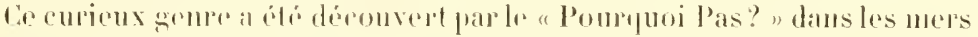

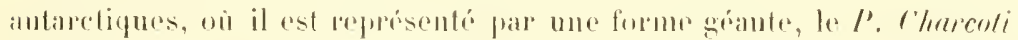

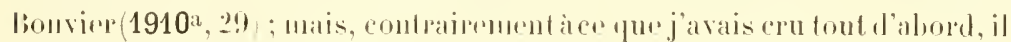
est cigalemonl répandu dans la zonr littorale des mor's chandes; sous le mon

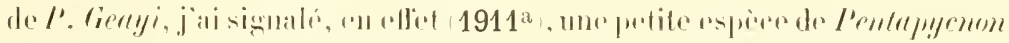

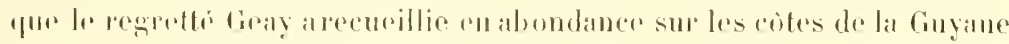

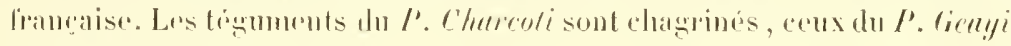
réticules.

Pentapyenon Charcoti li.-1. Bouvier.

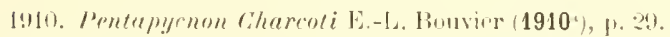

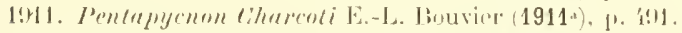

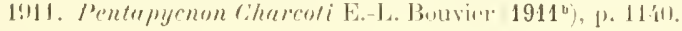

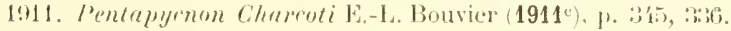

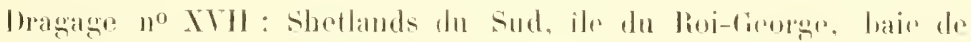

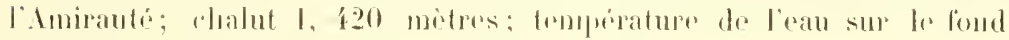
$+10^{0},: i$; vase, railloux.

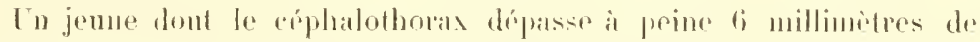

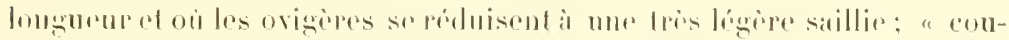
leur jaune légirement orange fris pàte n No Z26 i, completement dépionmentíe dans latcoul.

Un second jeune un pen plus avancé que le précrdent : Ir céphalothorax atteint 10 millimetres, mais les ovigiones ne sont pas plus díceloppes. La coloration, d'un "blen pàle ", serst quelque pen conservé dans l'ialeool No 727 .

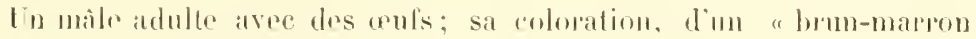
fonce "e est pereistante dans l'atcoul.

Les dimensions de cet exemplanire sont les suivantes: 
Diametre maximum de la trompe................ 3,6

Longneur du céphaton....................... 2,8

Diamétre maximum du céphalon. ................ 4,0

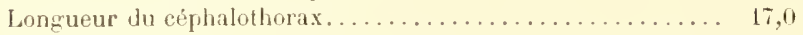

Largeur du trone entre ta hase des prolongements coxaux

(prattes?) ................. $i, i$

Largeur du tronc avee les j,rolongements coxaux (pattes :) . . . 10,0

Longueur de tabdonen ...................... $\quad \mathbf{1 , 0}$

Longueur totale des coxie de la pratte 2 droite......... 6,2

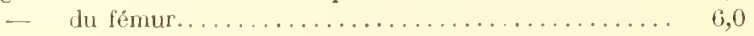

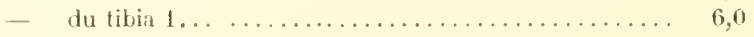

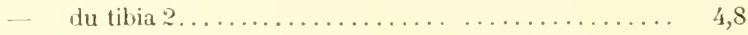

du tarse-propode..................... 4,0

- de la grilfe.......................

Colte remarquable rispice est dr beancoup la plus grande forme do toute la famille; mème ses deux jembes mentionnés plus baut présentent des limensions qui dépassent notablement colles de presque tous les autres Pyenogonidés. Nous allons d'abord décrire te mâle adulte, aprés quoi nous indiquerons les ditlérences observeses chez les jeunes.

Lanimal est partout recomvert de grambles irreguliers et contigus qui donnent à ses tóguments, vus sous la loupe, une appatence fincment

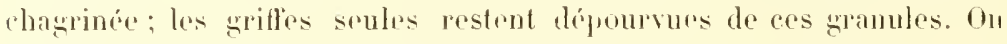
nobserve pas la moindre trace de rétirulation tans les téguments.

Lat trmmpe fig. 98, 99 est aussi lomgur que las trois premicrs segments

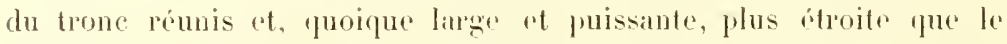
rephalon. Elle est un peu recourbée vers le has, ovolide dans ses deux firrs hasilaires, puis munie de trois gros tubereules obtus, un sur la face dorsale ef deux autres aux angles lacéro-infériems tans son tiers terminal, yui rst assez nettenent trifacié; vu den haut, le bord distal parait arrondi "t moirtre, an contraire des autres partios du corps, yui sont d'un lumn jaunater vu de face, il nontre un vaste orifice buccal triangulaire, an eentre duquel la frute de la bouche apparait triradiée.

Le rephlaton lig. 98, 99) est fort large; tries peu en arriere de sun

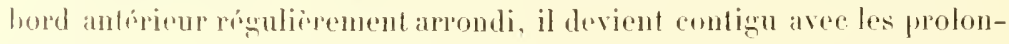

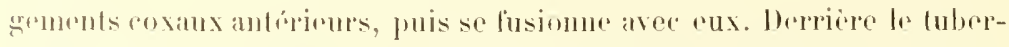
cule orenlaire, il presente une salillio dorsale plus réduite qui indigur, 


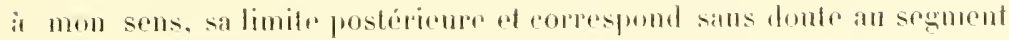

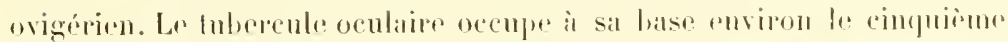
de la largeur du cáphalon: à peru près aussi haul que large, il se réfrecit jus-

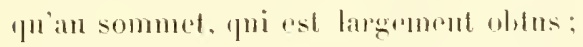
il est un peu romrex an arriege el sincline legiormont rn aval. Les gens

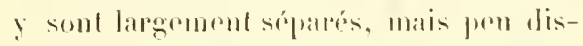
tincls, lour jigment noir ótanl localisésur un pertit areera forminal forl befroit.

Le trome fig. !x, !) rest font medtement articule; ses quator sogments anterioms se forminent en arriere par un bourer lot obtus, au confer dugued seblise mon tris fort tubereule arrondi; les trois poremiers segnents sont it pen press dr lan-

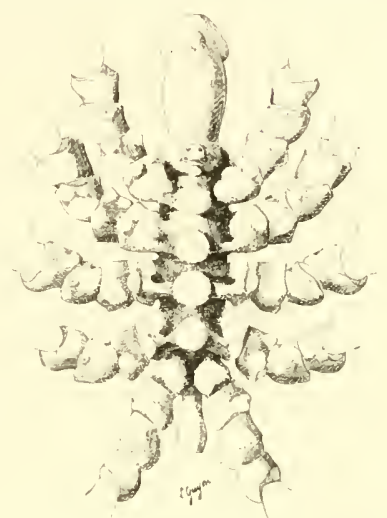

Fig. 98. - Pentaplenon Ciharenti Boux:

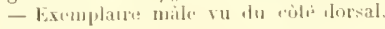
ir. 2.

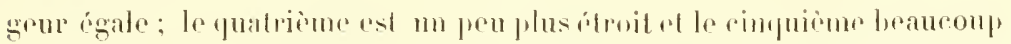

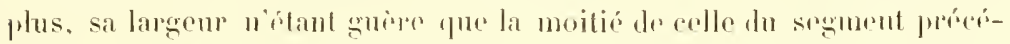
drent. La face veutralo rst prespue plate, sans lubereule, avere un fort sillon

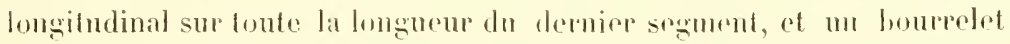

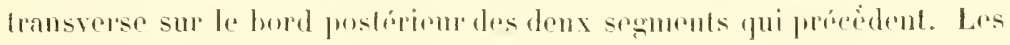
prolongements coxaux soml hien plus larges que Iongs laiblement dilatós dans leur partic topminale ot munis dorsalement diun fubereule arroudi près de lour bord. sur les probngemonts coxanx posterieurs, qui soul très coults. Ins deux fubereules présentent dro diureusions phus forles et ne sont séparós l'un de l'aufre que par une dípression étroile, mais profonde.

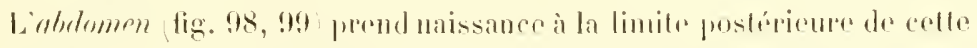

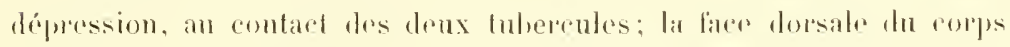
se continue réguliorement sur la siemme fropere. mais en dessous il

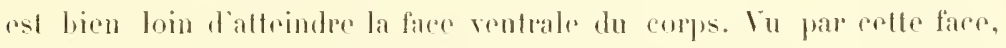
l'abdomen semble neltement articule, mais il neen est rien. comme on pent s'en convainere on examinant lanimal du crife du dos. Labdomen ast

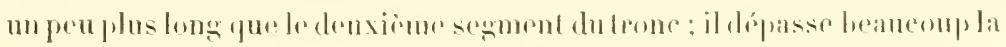

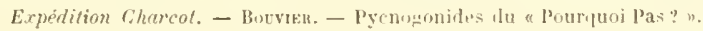


premire roxa; fris fortement dilation massme, il est beaneonp plus ítroil a sa hase fur dans sa partie: anale, fui est tronquée; sa plus grandr largeur est au nivean du bord distal dr la première coxa.

Les origripes lig. !!! se fixpul à la face ventrale du céphalon, sur une

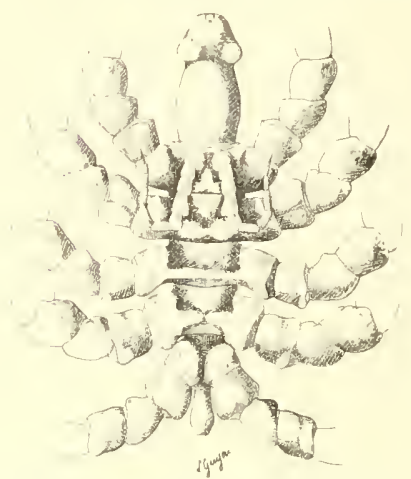

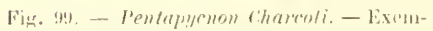
plate mảe ru du ciré inferieur. (ir. 2. paire de larģes mais légères saillies collliguès à leur base; leur longneur ost de lfmm, ,i; lenrs articles hasilaires soul assez lareses, mais, a partir dn troisiome, qui se dilate beancoup en avanl Jn eoté interne, lenr diamotre se rétrécit graduellement, ef le dixieme on demire a la forme d'un stylet. Les articles sont de longuenr subigale; pourtant lo troisième, le cinquième et le sixième sunt ligerement plus longs 'jue les autres; of le stylet terminal est un pu plas court que l’article précédent. Il y a cncore autour dos ovigìes les restes froissós du cément qui englubit lis anfs.

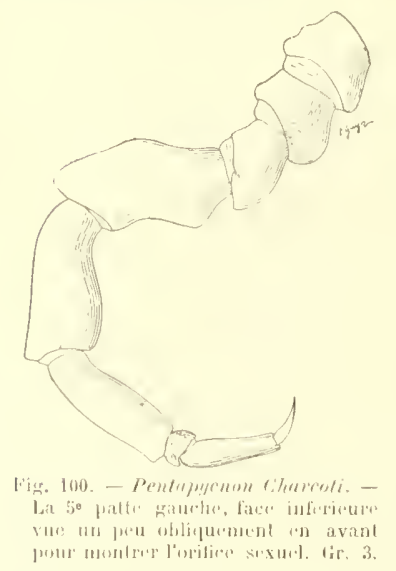

Les pattes (fig. 98, 99, 100) sont fortes ot issez longnes. Lemrs trois articles cosaux ont des longnemrs ì peu pris égiales, mais lenr langerur va en décroissant du premier au troisime. Pris de son bord distal, Ir prenier présento drux larges saillies obtuses, l'une antériome assez faible, l'autre postérienre plus élevée: lo druxiome est analegue, mais son tubereule antrírenr, fort réduit, présente à son sommet me trinte claire qui parait indipuer l'orifiee d'une glande (fig. 98; il on est ansi domoins dans les fuatre promières

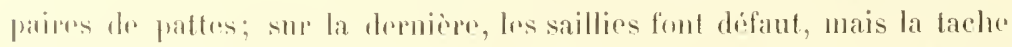
Waire reste fort nette; an surplus, on observe sur la face ventrale dr 
ce dernier article coxal une lonte voussure antero-externe, oil l'ou apereoil en avant l'orifice reproducteur, qui est pelit of sublriangulater

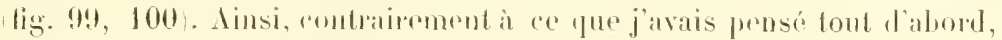
los oriliens sexuels milus soml localisis, commue dams les l'yrmoymmm, sur les pattes postérieures. Le troisiome artiche cosal ne présente rien de remaripuable.

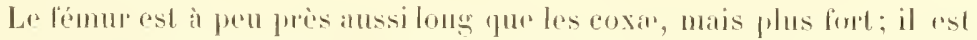

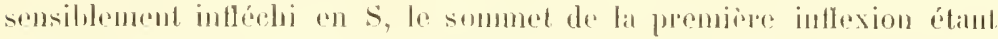

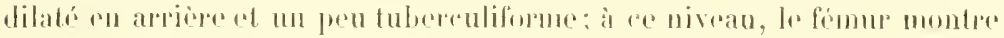

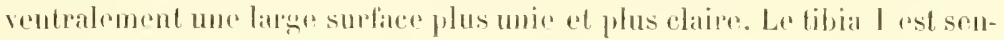

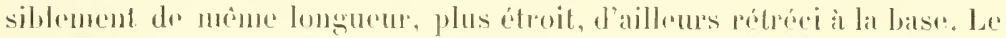

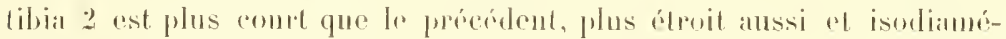
Iripur. Le Larse cost tris court, surtont du colé elersal; le propode esl

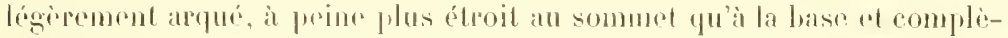

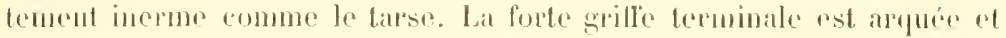
plus longue que la moitio du propode; il n'y a pas do grillos auxiliairos.

Trolle est la structure du màle adulte. Dans le plus pretit des jeunes

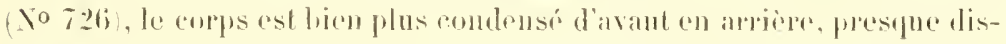

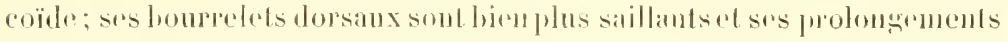

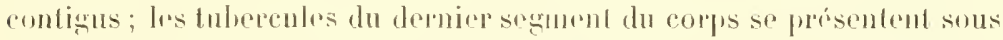
la forme de simples voussumes tries larges; rems des prolongentents coxaux, des coxa el de la tromper apparaissent a prine, mais la saillio" fémorale est tuluereuliforme; anfin les divers arliches des paltes sont plus eourts que clezl'adulte; l'aludomen est moinsmeltement en massmo et phus

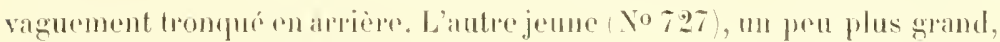

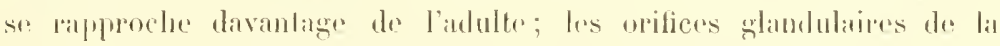
demxieme roxa sont hien nols, ef la tromper presente en avanl ses frois

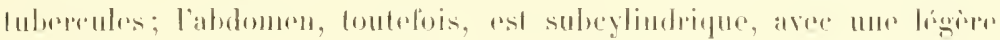

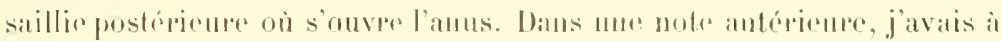
lort regande ces deux jeumes commone des findellus.

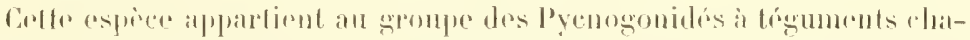

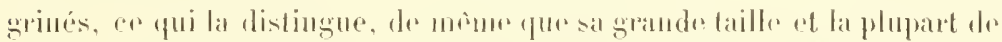

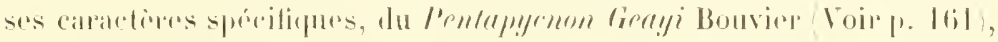

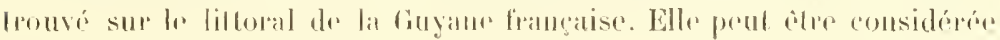


commo la plus lople trouvaille laite au cours de la campagne of méritail

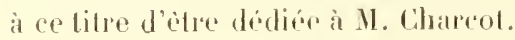

\section{Cienre Pycnogonum Brünrich.}

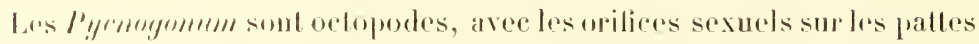

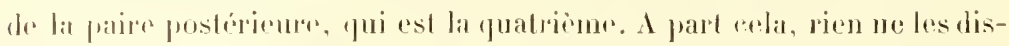

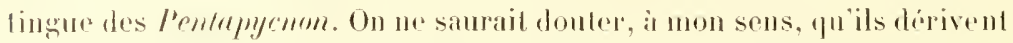

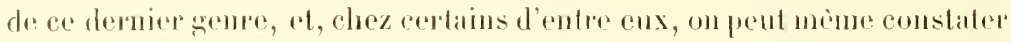

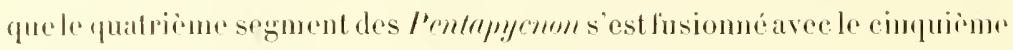
enferdant ses appendices; celle lusion semble maniluste dans le f'yrno-

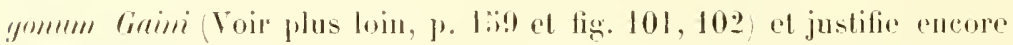
lo cantere primitil que nous athibuons aux l'yenogonidés décapodes;

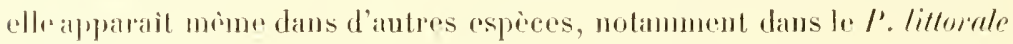
silör.

Les l'ycomgrmmm sont répandus dans toutes les mers, on ils habitent lat

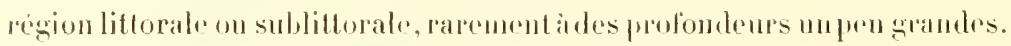

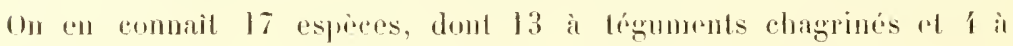
lóguments réticulés. Cont parmi les premiores que se frouvent les esprees pui frépuentent lo voisinago des pobles: 2arctiques et subaretiques,

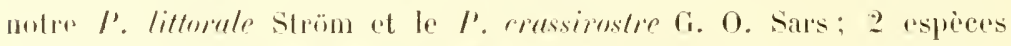

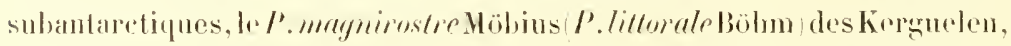

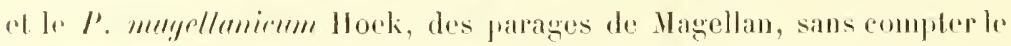

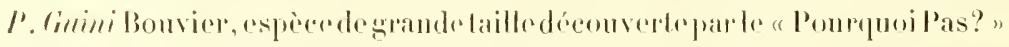

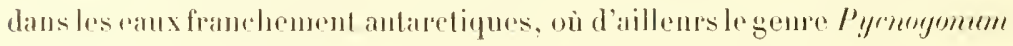
n'elait pas commu jusqu'ici.

\section{Pycnogonum Gaini E.-1. Bouvier.}

1:10. I'yenogonum Ginini E.-L. IBusvier $\left(1910^{2}\right)$, 1. 30.

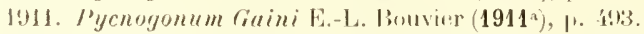

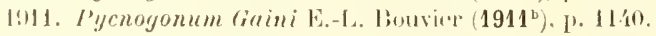

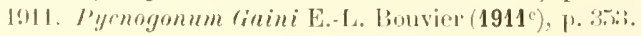

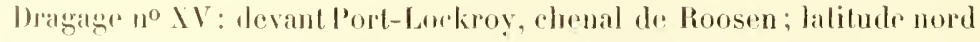

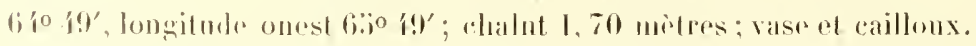

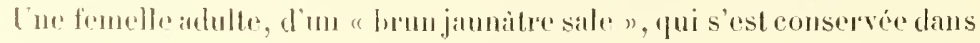




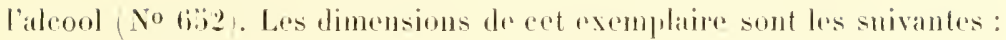

Millim.

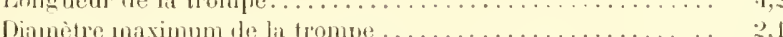

Longueur du céphalon .....................

Litrgeur maxima du cephalon.................. 3,1

Longueur du eéphalothorax.................... 10,2

Latrour du trone entre lit base des prolongements coxanx

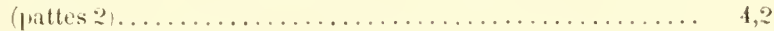

Largeur du trone avee les prolongements coxaux (pattes :2) ... 7,0

Longueur de liablomen...................... 3,0

Longutur totile des coxie de la patte 2 droite.......... $\quad 5,11$

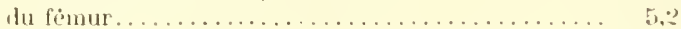

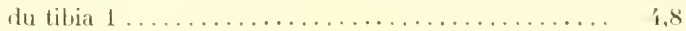

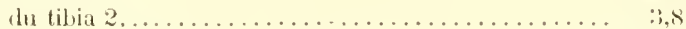

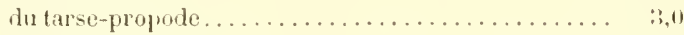

de la griffe...................... I,7

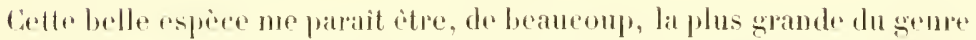

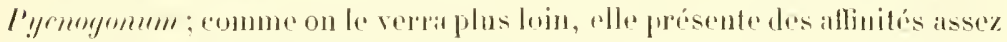

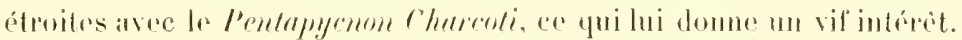

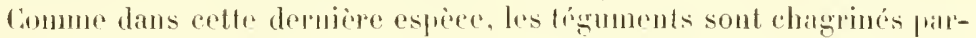
tout. saul sur les grilles ot an bout de la trompe, majs les geranules rui froduisont lit rugosite sumt peut-être un pen plus forts; au surplus, la roloration est prespeure la mine, mu pert moins brunc toutefois dans notre speicinten.

Lat trompe tig. 101-103) ast toutrofis tris diflérente ditls les adultes des deux especes; elle so dilate à proinc d'atrribere en avant sul sa moitié basilaipe daus le l’. fillmi, puis se rétrécit jusqu’a l'rxtrémití antérimpe, qui rst

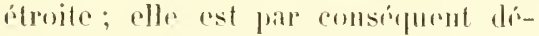
fmorve de lat dilatalion teminale mmnie de trois fubrecules qui caractérise si meftenuent lateompe du Pentrpyrenom

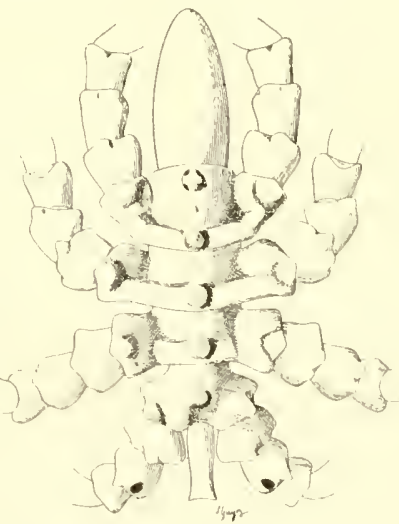

l'iy. 101. - l"yenogonum Giaini Bonv., ? Animal vu du cots dorsal avee les orifices sexurls. lir. $31 / 2$.

Cherenti, mais il faut ubserver que cefte dilatation est reduite, dams 
Ir plus jenue immature de l'espèce décapode, où d'ailleurs les tubereules sont ì peine indirués.

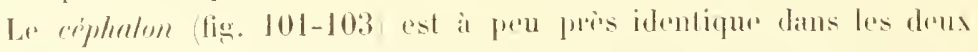
especes. foutefois nu peu moins siallant en avant dans le l'. Gomini, car

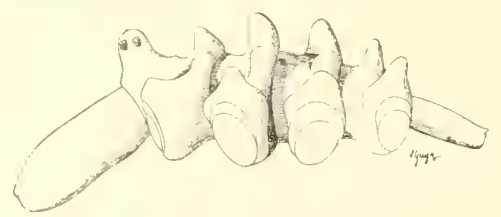

Fig. 10ะ. - Pycungonum fiaini, \&. - Animal vu du còte grizuche sans les pattes. Gr. $31 / 2$. it se fusionne plus vite aree les prolongements lateraux de la premiere paire. Les principales dillirences relatives à cette partie du corpssont l'atrojhir prespuecompletre, dans le l'. Ginini, du tulsercule éphatique postérieur et lat structure tout autre du tuberenle oculaire. Ce dermier est plus large dans

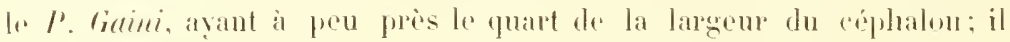
sonlargit un peu à la base parallelement an bord céplatiqur, puis conserve le même calibre pour se terminer en dòme au-dessus des yeux, qui sont petits et largement séparés, comme dans le l'cutapycnon charcoti. lei encore, les juunes de cette dernière espece ressembleut davantage an l'. Gaimi, tant par la forme on le développenent de leur tubereule oculaire que par la dilatation basale qu’ils présentent parallilement an bord antérieur.

Lי trme (fig. J01, 103) est constitué de mème dans les denx espèces, avec un fort bourelet arrondi sur le bord posterieur de tous les segments a l'exerption du derniev et un tubereule dorsalau milien de ee bourelet. Les probongements coxanx sont égatement identiques, are un gros tulerende dorsal arrondi pres de lem hord externe. La diflépence assentiefle entre les deux espeeces a trait anx tubercules dorsaux médiaux, qui sont en eone a sommet oblus dans lo l'. Cinini ed Jargement arrondis

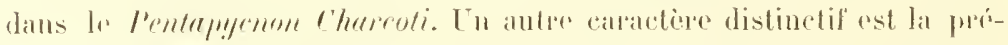
sence, dans to I'. Cimini, d'un tubereule dorsal médian sur le segment postérieur du corps fig. 101. 102 1, un peu en avant des deux lubereutes

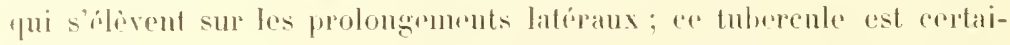
nement l'homologne du tubereule des segments yui procident et

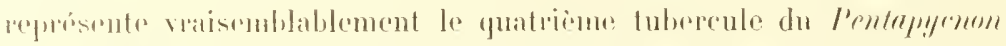
C'harenti tig. !s). De sorte que le I'ycnoyonum Graini posséderait in 


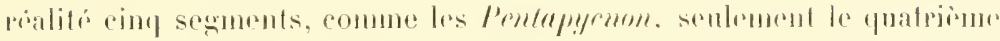

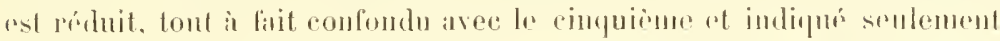
par son tubereule dorsal métanérique. Une disposition identipue s'observedansd'autres/'ycongnmm, nolamment dans mos deux esperees aretiques, $P$. reressimestere siars of P. littorale ström ; dalls er dernier, le souvenir mótanórifyur des Pentapycuon est même poussio plus loin que daus motre expecen, ear on retrouve lo tuberecule du segment. ovigérion silur "n arriere du tufrercule oculaire. Cresconstatations paraissent de nature it montrer

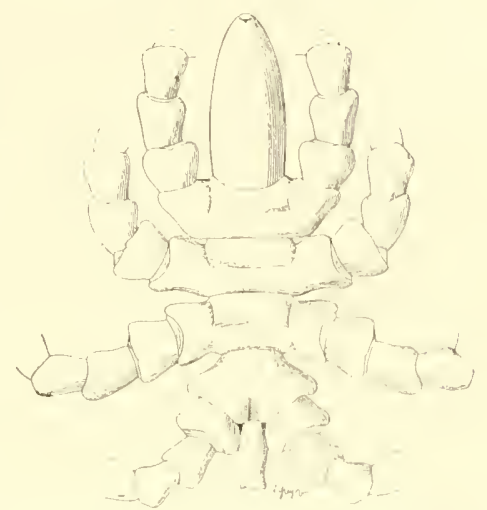

Fig. 103. - Pycnogonum tiaini, 8. - Animal su du coite rentral, sans les jattes. Gr. $31 / 2$. que los l'yenogonmm, espieces octopodes, dérivent des l'entapyrnom, qui sont décapodes (Yoir 11. 21-25). Labdrmeen (tig. 101, 103, do notre esperee dipasse lo milieu de la deuxième coxa des pattes postérieures ; il ust notablement comprimé daus le sons dorso-ventral, el à peu pres de meme largeur sur toute son étendue; son extrémitio postérieure est earróment trompuée. aree une légere saillie a chaque angte. On sait que sit dilatation teminale est tros firible dans le grand immature do Penlupycuon chareoti.

Les pattes (lig. I0 i) sout peu dillérentes dans les deux especes; la promiripe coxa porte deux tubereules dorstux, mais la deuxième, qui est un preu plus longue, n'a

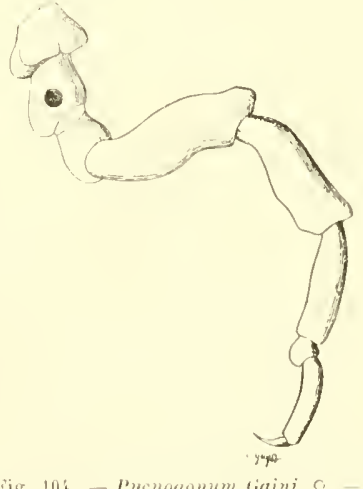

ligr. 10t. - Plycungonmm tiaini. S.Lat fo fiatte droile aver l'orilier. siviuel. fir. $31 / 2$. 'qu'un seul tuhercule ou se voit, sur certilines pattes, un lache claire; le grand orifice lemelle, arrondi, est situr an arriere de cre tubereule,

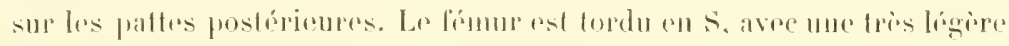


dilatation pusterienre an nivean de la pemiere courbure, of mo asser forte saillie obluse dans la partir dorsale, au-dessus de l'articulation du tilia l. Les autres articles sont de même forme que ceux du P'entapycron Cluarcoti et présentent des longueurs à peu prìs analogues.

Notro espèce appartiont au grompe des l'yromgomem on les téguments ue prósenfunt pas do dessins en réseanx, mais sont chagrinés. Elle so

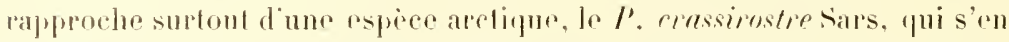
distingurs surtout par sil pelite taille, son abdomen arrondi en arriere. ses tuberenles dorsaux muins flevés of la saille plus forte du fémme. II" y a un léger tuberentr post-oculaire sur le cóplaton dans cette dernière aspeer (Voir Sars, 1891, fis. 2a); ce lubereule ast beaucoup moins saillant "fue dans une antre esperee aretique voisine, le $P$. littorale Ström, mais il a totalement disparu dans notre esjere. En tout cas, cumme je l'ai dit phus haut, les trois espeeses se rapprochent du l'entapychom E'hareoti par la présenee d'un bubereule médian sur le segment qui parait terminal lig. 101, 102 . le tulereule fail défaul dans une esprece californienne également tries voisine, le P. Stearnsi lvers.

En fail, malgré des différences assez grandes dansla forme de la trompe, 10 I'. Ciami parait dériver du l'entapyenon Claarcoti par suppression des appendices du quatrieme segment de cefte espece, rédnction et fusion de ere segment avee le dernicr. Les deux formes se rapprochent mon soulement par leur structure, mais anssi par leur grande taille, qui les lail placer parmi les géants dans la famille des Pyenogonidés.

\section{API'ENDICE}

Afin de meller au courant nos comnaissances sur le gemre l'entapycnon

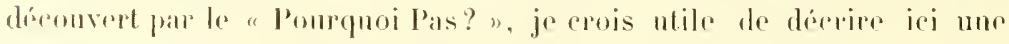

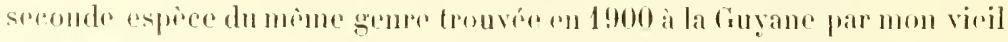

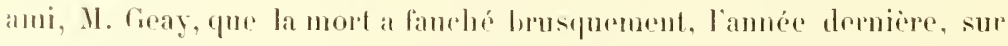

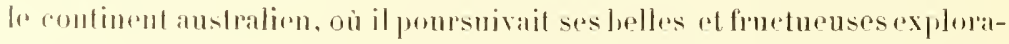
lioms sejentiliques. J'ai fail connaitre cette espece sous le nom de l'enta-

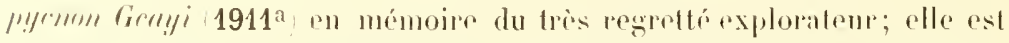

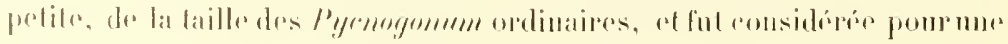


forne de ce derniep senre quand elle entra dans les collections du Musrum ; je regrette vivement cette confusion, qui a privé nonn vieil ami du prand plaisir de voir son nom allaché à la découverte du premier l'yenogonide décapode véritablement inconteste. Sans doute, to grenre Jecolopoda fut décrit par Eights à une date birn plus ancienuo (1834, mais

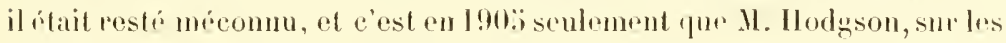
indications de M. Cahman, révéla aux savants la déeouverte d'Eights. L'année précédente, en 190\%, le mème naturaliste lit connaitre le genre l'enturymphon, trouvé par la " Diseovery "dans la baie Mac-llurdo, ed cerst en 1900 que M. Geay captura en fiuyanc la eurieuse espice dont on va lise la deseription.

Pentapycnon Geayi L.-L. Bouvier.

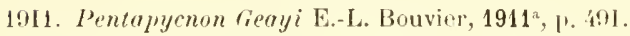

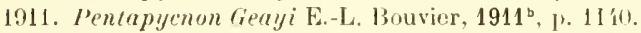

1911. Pentrpycnon Feayi E.-L. Bouvier, 1911 ${ }^{\circ}$, p. 35:3.

Cefte espece lut trouvé par Geay à la Guyane française, "parmi les Hydraires ", oì elle doit etr" phutit commune, les spicimens recucillis

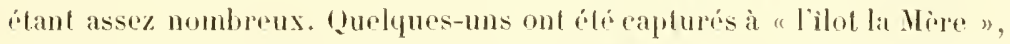
qui se trouve au large de l'embouchura du Mahury, vers le sud-est de Ciagenne; les autres provirmmont stoment do la eote dans la rógion de Ciayenne, encore que leur étiquelte d'origine porto tout simplement pour mention "Guyane fran!aise ".

L'espece presentr la taillo ot l'aspect des l'yemogmmm les phus normaux. Voici les dimensions prineipales diune lemelle.

\begin{tabular}{|c|c|}
\hline Longueur de la trompe. & $\begin{array}{l}\text { M1LIm. } \\
2,5\end{array}$ \\
\hline Diametre maximum de la trompe.... & 0,6 \\
\hline Longueur du éphalon....................... & $0,6,5$ \\
\hline Diametre maximum du céphalon . . . . . . . . . . . . . . . & 1,2 \\
\hline 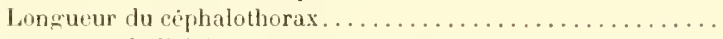 & 2,7 \\
\hline 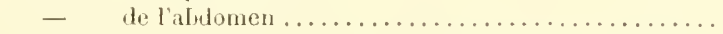 & 0,8 \\
\hline $\begin{array}{l}\text { Largeur du trone entre la base des frolongements coxaux } \\
\text { (patte }:) \ldots \ldots \ldots \ldots \ldots \ldots \ldots \ldots \ldots \ldots \ldots \ldots \ldots \ldots \ldots \ldots \ldots \ldots \ldots \ldots \ldots\end{array}$ & 1,1 \\
\hline Largeur du trone a vec les prolongements coxaux (jatte 2$)$. ... & $2, \because$ \\
\hline Longueur totale des coxie de la : ${ }^{\circ}$ ratte droite............. & 1,45 \\
\hline - lu fémur de cette patte................ & 1,25 \\
\hline 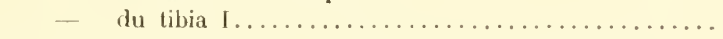 & 1,30 \\
\hline
\end{tabular}




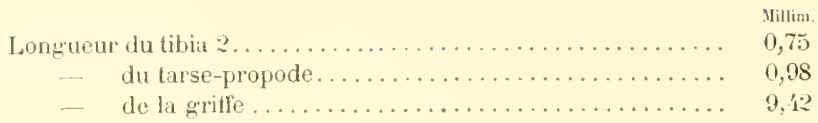

Les mailes sont en général un peu phus petits que les femelles.

Les triguments de l'animal paraissent unis an premier abord, mais its ont, on lait, lasurface finement irréguliere et présentent une infinité de lingires sullies dtroitement contiguës; les grilles seules sont à peu près lisses. [ne riche réticulation (1ig. 10\%-108) apparait dans toutro les partirs du corps, saul sur le tarse, le propode et la gritle des pattes, sauf

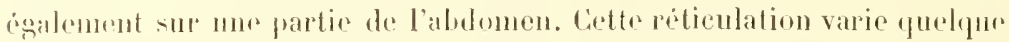
pen suivant las exemplaires, mais ses principanx dessins pestent constants.

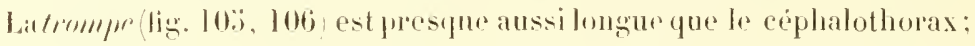
ello a la forme d’un frone de cone à sommet ohtus et so rétrécit rógulièrement de la lowe in lextrómité distale. Les mailles de sat réticulationse rétrécissent ut devienment vagues ou mulles dins sa moilié la plus étroitr, pour

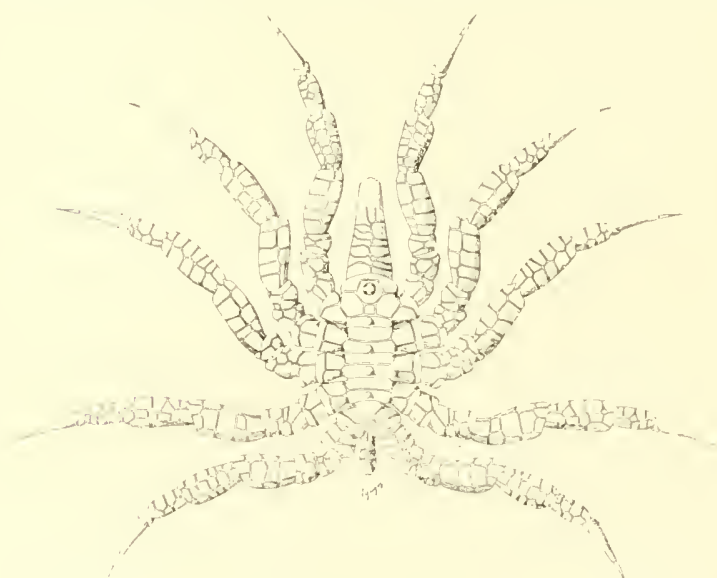

Fig. 10is. - Pentapycuon fieayi Buuv., f. - Animal vu du cote dorsal avec l'oritiec sexuet. Gr. $61 / 2$. disparaitre sur la ligne médianedans ecter rógion; à la lase, elles sont très mettes et disposeres à droite ot à wallche diune ligne dorsale médiane. Le céphrolonn tig. 10:i, 106i) ast un pen plus large que la partie basalo de la trompe ; som bord antérieur et ses cotés sont lé-

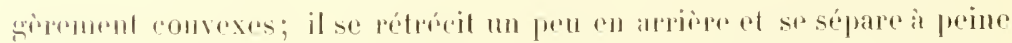

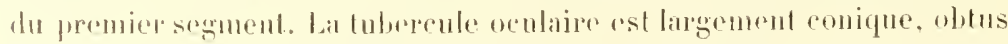

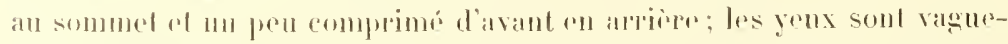
ment diflérenciés et à peu pres contigus; ceux de la paire postérieure 


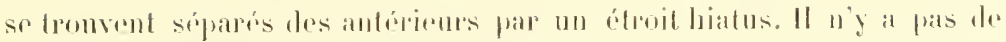

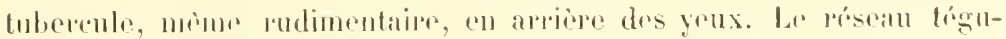
mentaine présento trois mailles principales, lone impaipe, polygonale,

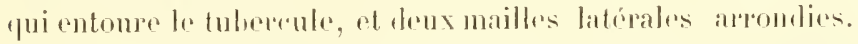

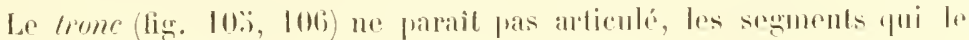
comprosont nétanl pas mobilos les mos sur los autres. Du cití dorsal, to lord pustí rieur de chapue sognownt siblere un prom en bourelot transversal ef prisente au milien un tubereule coniyue à sommed oblus; in avant of on arrière du bourreler, la réti-

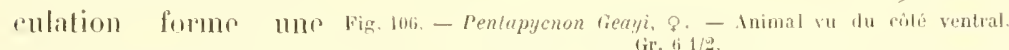
ligne transversale sur
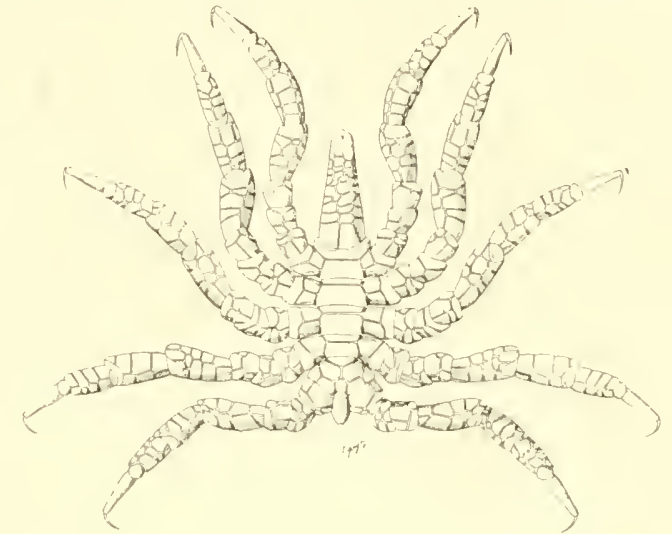

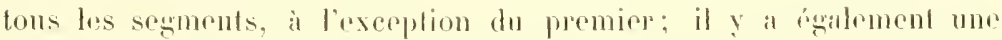

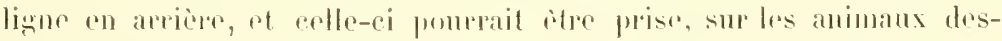

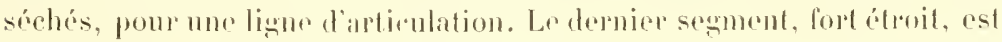

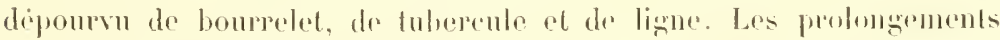
coxaux sont contigus el se dilatont mu pen de la base an sommet: leurs

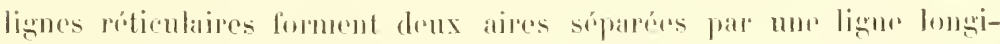

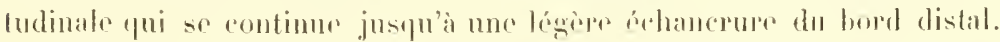

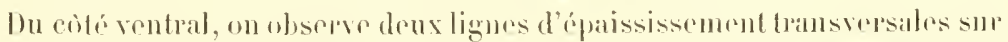

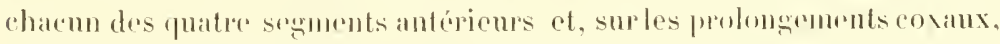

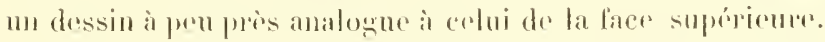

Labdomen est ovö̈de, un pen beticule et satss artienhtion hasale; il se dirige homizontalement en arriope of dépasse lo premier andele coxal des pattes posterpieures.

Les puttes tig. I0:i-los des cind baires sont subrigales ef semblables, sanf quelques dillópeners dams la disposition des mailles du resean ; 
Jemr surface n'est pas trós réguliere, mais on n'y observe pas de tubereules, sanf une paire de très légers an bout supérienr distal du fémur ot du

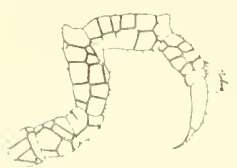

Figr. 10T. — Jentap?cnon tieayi. \&.- Fin: antirieure de la gr fallo droile. Gr. $61 / 2$.

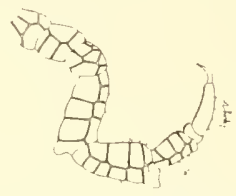

Fig. 108 - Pentapycnon reayi, 9. - Face pustiriture le la ge pralle druile Gr. $61 / 2$. premier tibia, el quelques faibles saillies aignës sur le hord infórieur da fémur. Lemr région coxale est un pen plus longue que le fémur, lequel est luimême lingirement plas court que le premier tilia: res deux derniers articles sont plus dilalés que fr.s suivants; le tarse est tris court, le propode un peu phus long que le tibia 2, lequel égale environ les deux tiers dn libia l. La grifle est un peu plus longure que la moitió du propode el dépourve de grifles auxiliaires. Le réseau de ces articles se compose de grandrs mailles plus on moins quadrangulaires que sépare une ligne longiludinalo dorsale, de chaque eòté une ligne latérale et, sur plusieurs articles, une ligne ventrale; ces lignes sont irríguliires, comme les mailles, ol se complifuent un pelu sur la face dorsale du second tibia. Le réseau disparait tolatement sur le tarse, le propode ot la grille.

Liorifier spruel (lig. 10.̈) de la frmelle est situé dorsalement prìs du bord posterieur do l'artiele 2 coxal do la depniere paire do patles.

L'orifice du màlre, phus petit, occupe la fice ventrale du même article. Les arigeres ligg. 109) sont courls et se comprosent de lunit articles y

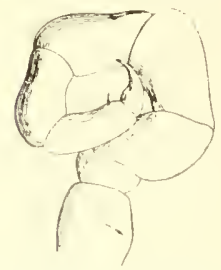

Fis. 109. - Pentapyrmon

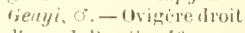
d'un alulte. fir. its. compris la grille terminale; très ćloignés l'un de l'autre à leur base, ils premuent naissance an bord postéro-exterue du céphaton, contre le prolongement coxal des pattes antérieures. Leur article basal est ovoüde el leaucoup plus grand que le suivant; il est sans doutr formé par la fusion de deux arlicles, car j’y ai ru, dans un spécimen, les traces d'une ligne arliculare; l'article + est un peu plus long que les articles 3, 5, b ef 7 ; ce dernier présente mo soire sur son bord interne. La grille est un peu plus courte que l'anticle 7. Le diamedpe des outs vario autour de $100 \%$. 


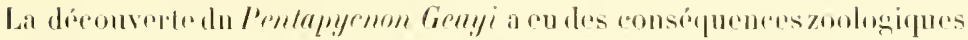
intéressantes. Elle a montrí lo que les Pyenugonides dérapoles ne sont pas localisés dius lus mers arctipues, comme je le pensais moimime ol comme on le eroyat jusqualen's 20 jur les deux esperes du genre l'entapycnonne sont pas an mìne degré d'évolution, le l'. Remyi,

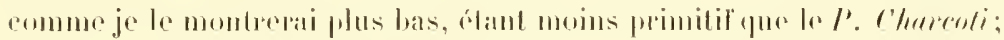

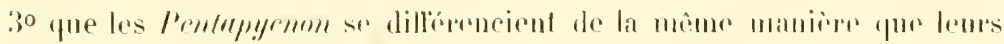

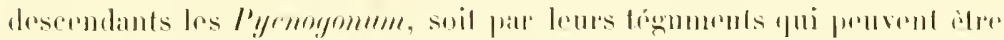

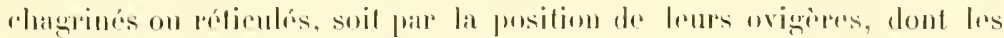

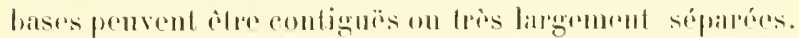

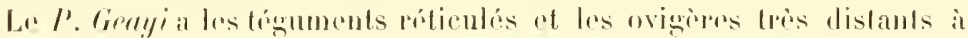

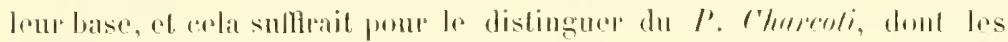

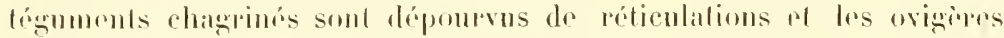

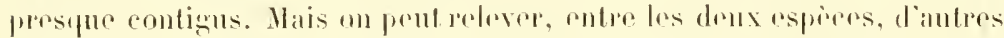
dillépences, of eolles-ei prósenlent une sisnification plus erande, parer

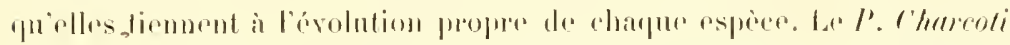

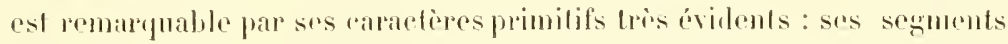
probifimes sont articulés et tris mobiles les wus sur les antres; son

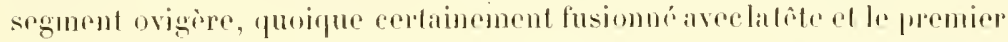
segment pédilipe, lajpelle encore les segments du fronc par la présence d'un petit tuberoule dorsal silurenarriere duluberente oculater ; rntin les ovigères emx-mèmes sont primitifs en ce sens quils complent un wrancl nombre diarticles, 10 an tont, y compris la grille forminalr. Dims le

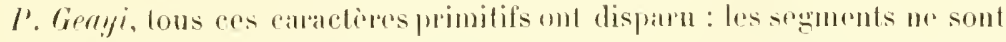
plus articulés; le tubereule ovigène a disparn, ef los ovignires ne comprennent plus que huit articles ; l'espece so trouve pare consépuent a un

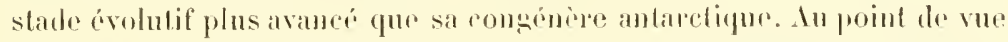

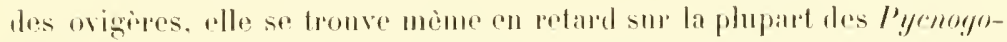
mum, puisque ces derniers présentent généralement, simon toujums, nenf articles ovigériens an lien de huit.

Le l'. C'hereoli est en outre remandyuable par sil grande taille, tandis 'pue le I'. Geayji a des dimensions péduiles ef ressemble an cela anx

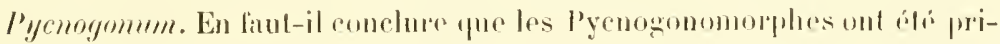

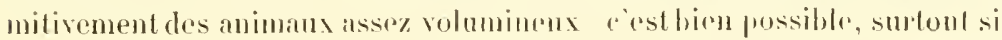




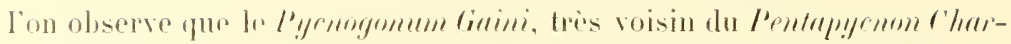
refi, estbeatuenup plus spand que les autres especes du gente. Mais on sait yur le gigantisme affectrassez souvent les animaux des mers glaciales, ef cela nous oblige à n’avaneer qu'avec précaution la conclusion qui précinde. 


\section{IXDEX BIBLIOGRIPHIQUE}

(Les numeros sans astérisque ont seuls trait ì des espress unturctiques.)

1912. Dele (F. IEfFues). - A general Notice of the hiological Memoir's (Nat. untaret.

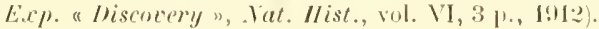

1S79. Bunn (R.). - Ueber die l'yenogoniden des könighl, zoologischen Museuns zu Berlin, inslesondere über die von S. M. S. "liazellit " mitgelomaten Arten

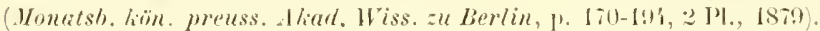

1:R.). Botven E.-I.). - Olservation prétiminaires sur les Pyenogronides recueillis daus la région antaretipue par la mission du "Franeais" (Bull. Mus. d"Mist. nat., 1915, 1. $29 \cdot 4=297 \%$.

19kr-. ID. - Nourelles observations sur les l'yrnogonides recueillis dans les régions antaretiques au eours de la campagne dirige par Ml. Jean Charcol Cumptes rendus Lrat, des sciences, 1. CXILII, J. 15-22, 1906).

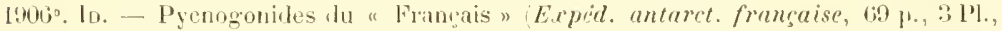
is tig. dans le texte, 19uri).

$1910^{2}$. In. - Les Pyenogonides ì ring paires de pattes recueillis par ka Mission antarctique Jean Chareot à bor du "Pourquoi las?" (Comptes rendus Acad. des sriences, t. CLI, 11. 20-32, 1910).

$1910^{\mathrm{b}}$. 10. - Note sur les Arthropodes marins recueillis par M. Rallier du baty aux iles kerweten (Bull. Mus. d'llist. nat., 1910, p. 178-17!), 1910).

$1911^{\mathrm{a}}$. Io. - Uliservations sur les l’yenogonomorphes el principalement sur le l'entupycnon Cieayi. espice tropicale à dix pattes ciomples rendus dcal. tes sciences, t. CLII, 1. 1133-114:, 1!11].

$191 \mathrm{I}^{\mathrm{b}}$. ID. - Les Pyenogonides du "Pourquoj Pas?" Comptes rendus .Icad. des scienres, 1. CLII. P. 1136-114:, 1911).

1:11 ${ }^{c}$. In. - Les l'yenogonides décapodes et la classitication des Pycnogonides Congrès international dentomoloyie, Bruxelles, 1910, p. 345-35.), 1911).

19l1d. lo. - Algunos anmales marinos de las Islas Sandwich. Pyenogonides (Ann. Huseu Yac. Buenos-1yres, 1. XXI, ]. 413, 111, 1911.

19og. Cialman (W. T.). - The Prohlem of the Pyenogonous (Science I'rogress. $1 !(0 ! 1)$.

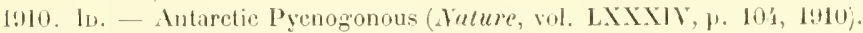

"1893. C.anpexten (i. 11.). - Reports on the Zoological Collections male in Torres Straits by l'rolessor A. C. Hadton, ISSS-18s9. Pyenogonilit (Supplement)| Proc. roy. Soc. Dublin (N. S.), 1111, p. 21-27, P. 11, 189:3).

18:4. In. - Pyenogonida from Killala Bay Proc. roy. Soc. Dublin (N. S.) Y, 189.1 .

190.). ID. - Notes on the segmentation and Phylogeny of the Anthropoda, with an account of the maxillae in Polyrenus lagurus (Guat. Journ. Mic. Ste, L. XIIX, 1\% 40?-191, P. XXVII, 1905).

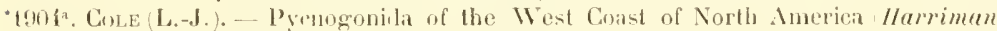

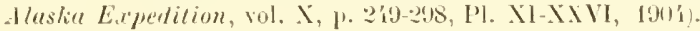


"tonǵb. Cone. - I'yenogonida collerterl at Bermuda in the Summer of 1903 I'roc. Boston

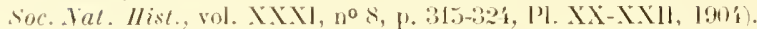

1905. ID. - Ten-legred l'yenogonius, with lemarks on the Classitication of the Preno-

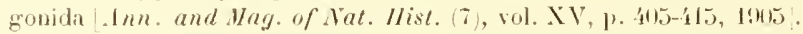

"16)o. ID. - Reprorts on the scientilic results of the Expedition... " Albatross ". Pyeno-

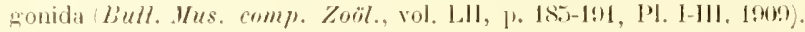

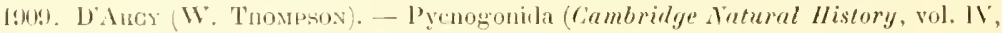

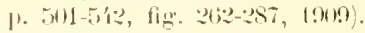

"18s1. Dumitax (Anton). - Die Panlopoden des Ciolfes von Neaprel Fauna und Flora des golfes un leapel, III Monogr.; $252 \mathrm{p}$. et $17 \mathrm{Jl}, 1881$ ).

1834. Engnts J.). - Deseription of a new Animal helonging to the Arachnides of Iatreille, discovered in the Sea along the shores of the New Shethut Islands (Boston fourn. Mat. Mist., vol. I, 1. 203-306, M. VII, 1834-1837).

190.2. Hodgson (T. V.). - Pyenogonida ( Southern Cross "Exp., 1. 256-258, 19k\%). 1904. Lo. - Un a new Pyenogonid from the South Polar liegions inn. and Nay. Nat.

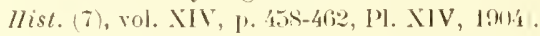

1905*. 1D. - Scotia collections. On Deculopodu australis Eights an old Pycnogonid rediscovered ('roe, roy. phys, Soe. Edinburgh, vol. XVI, part. 1, 1. 35-4?, Pl. III, 1905).

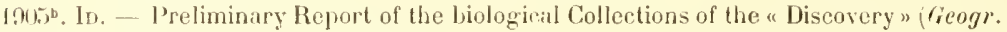
Joum., p. 39i:-4in), 1905!.

190rac. 1D. - Decaloporla and Colossendeis (Zool. Anz., 13, XXIX, p. 254-256, 1905).

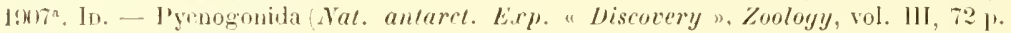
et $101 \%$., 19017 .

1:kitb. In. - Pyenogoniden Ergeb. Mamburg. Magalhaenische Sammelreise, :20 I. et 6 tig. dans le texte, 1907).

19Ms. In. - The Pyenngonida of the Seotlish aational antaretic Expedition (Trans, roy. soc. Edinburgh, vol. XIN1, patt, I, 1. 159-185, 3 P1., 1948).

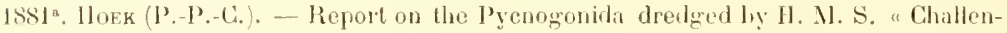
ger $)$ during the years $1873-1876$ (Challenger lieport, Zowl., vol. 111, 1iz j... $\because 1,1,1881)$.

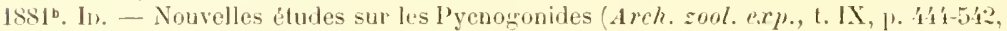
Pl. XXIII-XXX, 1881 .

18:8. ID. - On four Pyenogonids dredged during the Cruise of the "Challenger " Tijdschened. Dierk, Jereening, vol. V, 1. 2!H1-301, I'l. II el III, IS!S).

19M. Londan J.-G.-C.). - Decoloporta Eights oder Colossendeis Jar\% (Zool. Anz.,

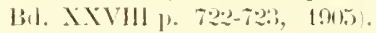

ISMS. In. - bie Pantoponden der Siboga-Expedition (Siboga-Expeditie, Monog. XL, S8 11. et $15 \mathrm{Hl}$., 19)(18).

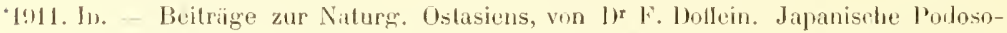
mata (Abh. math.-phys. il. der h. Bayer. Aliad. IJiss., II. surjl., Bil. IV, 7. 1-18, Tal. I, II, I!)11).

"t91: ID. - Note préliminaire sur les " P’odosonati » (Pyrnogronides) du Musée océanographique de Monaco (liull. Inst. wcéan., no 2is, p. 1-1 1, 1912).

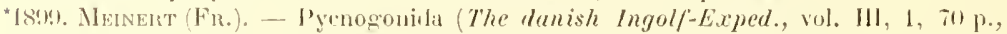
5 , $1 ., 1809)$.

$18755^{2}$. Mlens (ED.). - Descriptions of new sifecies of Crustacea collected at kergue-

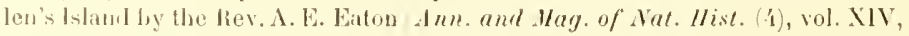
1. $73-76,1825 \%$. 
1875, Mlens (ED.). - Descriptions of thres adlitional species of Cirusfirea from Kergwelen's Lam and Crozed Istand with Rematks upon the genns l'aramaera

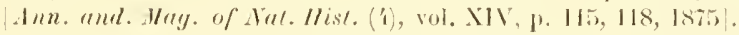

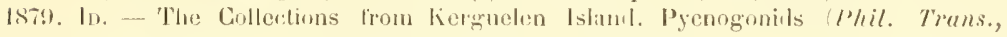
vol. CiLXVIII, 1, 211-214, P1. X1, fig. 7, 8, 5, 187!).

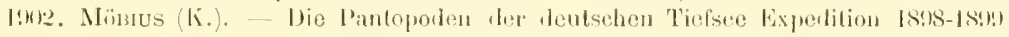

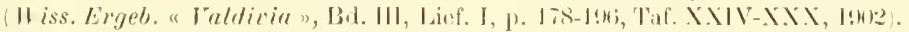

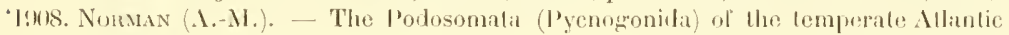
anl Arctic Oceans (Jumrn. Iinn. Soc., Zool., vol. XXX, p. Is7-2:3s, Pl. XXIX, $19(\mathrm{M})$.

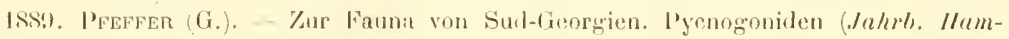
bury. Iliss. Anstull., dahrer. VI, 2te llibllte, 1. 11-19, 18891).

"Isl'́. Lexch. - Zoological Mliscellany, $\mathrm{V}, 1$.

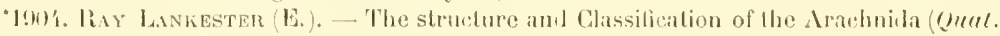

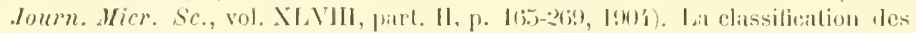
P'yenogonides, dans ce mémoire, est l'esuvre de .l. locock.

"I891. Sars (G. O.). - The Norwegrian Norll Allantie Expedition 18i6-1sis; Pyenogonida, 1891 .

18S7. Schnkewesscia (W.). - Sur les Pantoporles de l'expédition du "Vettor Pisan ", (Znol. Anz., dalurg. X, 1. 271, 272, 1587).

ISS?. JD. - Sur les Pantopodes recueillis par M. te lientenant fi. Chierehia pendant le

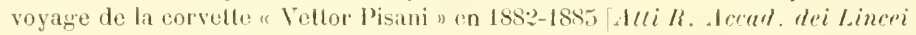
(IV), vol. VI. 1. 32:-347, une pl., Iss?! .

1902-03. Stembinci (T. R. R.). - The Noholies, a Sea-faring Family finowlerlge, vol. XXV (1902), 1. 37-40, 73-76, 1:37-1 10, 185-18!) ; vol. XXVI (19(3:), 1. 1'1-17, 14.

"19(M). Verrill (A. E.). - Adlitions to llie Crustacea and Pyrnogoniala of the Bermulas (Trans. Connecticul Acal., vol. X, part 2: Pycnomonida, p. 5siso-58:2).

1880. Wilson E. B.). - Reprort on the Pyenogonils of New Eugland and adjisent

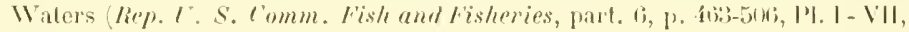
$188(0)$. 




. 

